MASTER

\title{
Summaries of FY 1977 Research in High Energy Physics
}

U.S. Department of Energy Division of High Energy and Nuclear Physics

October 1977 


\section{DISCLAIMER}

This report was prepared as an account of work sponsored by an agency of the United States Government. Neither the United States Government nor any agency Thereof, nor any of their employees, makes any warranty, express or implied, or assumes any legal liability or responsibility for the accuracy, completeness, or usefulness of any information, apparatus, product, or process disclosed, or represents that its use would not infringe privately owned rights. Reference herein to any specific commercial product, process, or service by trade name, trademark, manufacturer, or otherwise does not necessarily constitute or imply its endorsement, recommendation, or favoring by the United States Government or any agency thereof. The views and opinions of authors expressed herein do not necessarily state or reflect those of the United States Government or any agency thereof. 


\section{DISCLAIMER}

Portions of this document may be illegible in electronic image products. Images are produced from the best available original document. 


\section{Summaries of FY 1977 Research in High Energy Physics}
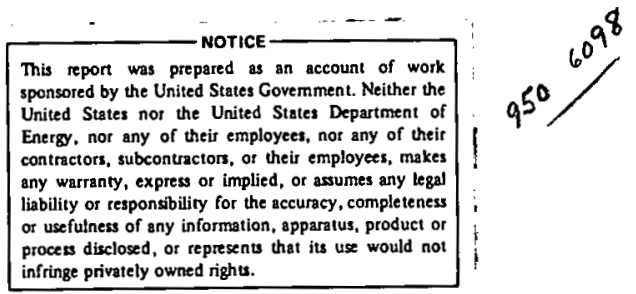

$\checkmark$

U.S. Department of Energy Division of High Energy and Nuclear Physics

October 1977 


\section{NOTICE}

This report was prepared as an account of work sponsored by the United States Government. Neither the United States nor the United States Department of Energy, nor any of their employees, nor any of their contractors, subcuntiacluis, ur their employees, makes any warranty, express or implied, or assumes any legal liability or responsibility for the accuracy, completeness or usefulness of any information, apparatus, product or process disclosed, or represents that its use would not infringe privately owned rights.

Avellable from:

Natiunal Technical Information Service (NTIS)

U.S. Department of Commerce

5285 Port Royal Road

Springfield, Virginia 22161

\section{Price: \\ Printed Copy: \\ $\$ 10.75$ \\ Microf Iche: \\ $\$ 3.00$}




\section{FOREWORD}

The U.S. Department of Energy, through the Office of Energy Research and the Division of High Energy and Nuclear Physics, provides approximately $90 \%$ of the total federal support for high energy physics research effort in the United States. The High Energy Physics Program primarily utilizes four major U.S. high energy accelerator facilities and over 50 universities under contract to do experimental and theoretical investigations on the properties, structure and transformation of matter and energy in their most basic forms.

This compilation of research summaries is intended to present a convenient report of the scope and nature of high energy physics research presently funded by the U.S. Department of Energy. The areas covered include: 1) conception, design, construction and operation of particle accelerators; 2) experimental research using the accelerators and ancillary equipment; 3) theoretical research; and 4) research and development programs to advance accelerator technology, particle detector systems, and data analysis capabilities. Major concepts and experimental facts in high energy physics have recently been discovered which have the promise of unifying the fundamental forces and of understanding the basic nature of matter and energy.

The summaries contained in this document have been reproduced in essentially the form submitted by contractors as of January 1977, and have been edited only for format and clerical errors. 
THIS PAGE

\section{WAS INTENTIONALLY LEFT BLANK}


I. Major Accelerator Laboratories

Argonne National Laboratory 2

Brookhaven National Laboratory $\quad 17$

Fermi National Accelerator Laboratory $\quad 42$

Lawrence Berkeley Laboratory $\quad 64$

Stanford Linear Accelerator Center $\quad 86$

II. Financial Plan Programs

Ames Laboratory 116

Calffornia, University of - San Diego 119

California Institute of Technology 121

Carnegie Mellon University. 129

Harvard University 133

Illinois, University of 141

Massachusetts Institute of Technology . 143

Michigan, University of 150

Oak Ridge National Laboratory $\quad 156$

Pennsylvania, University of 158

Princeton University 160

Rochester, University of 162

Wisconsin, University of 166

Yale University . 171

III. Other Programs

Adelphi University $\quad 176$

Brandeis University 178

Brown University 180

California, University of - Davis 183

California, University of - Irvine (2) 186

California, University of - Los Angeles 190

California, University of - Riverside 192

California, University of - Santa Barbara 193

California, University of - Santa Cruz 195

Cincinnati, University of 196

Colorado, University of 197

Columbia, University of 201

Duke University 203

Florida State University . 204

Georgia, University of 207 
Page Number

$\begin{array}{ll}\text { Harvard University } & 208 \\ \text { Hawaii, University of } & 210 \\ \text { Indiana University } & 212 \\ \text { Institute for Advanced Study } & 214 \\ \text { Johns Hopkins University } & 215 \\ \text { Lehigh University } & 217 \\ \text { Maryland, University of } & 218 \\ \text { Massachusetts, University of } & 219 \\ \text { Minnesota, University of } & 220 \\ \text { Northwestern University } & 222 \\ \text { Ohio State University } & 224 \\ \text { Oklahoma State University } & 226 \\ \text { Oregon, Univercity of } & 228 \\ \text { Physics International Company } & 230 \\ \text { Purdue University } & 231 \\ \text { Rice University } & 235 \\ \text { Rockefeller University } & 237 \\ \text { Stanford Univeristy (3) } & 240 \\ \text { Syracuse University } & 244 \\ \text { Tennessee, University of } & 246 \\ \text { Texas, University of } & 247 \\ \text { Tufts University } & 249 \\ \text { Washington, University of } & 252 \\ \text { Wayne State University } & 253\end{array}$


PART I

MAJOR ACCELERATOR LABORATORIES 
ARGONNE NATIONAI LABORATORY

Argonne, Illinois

Contract No. W-31-109-ENG-38 Supplement 18

Title of Project: Spectrometer Group

Person in Charge: R. Diebold

Sclentific and Professional Staff: D. Ayres, D. Cohen, S. Kramer, A. Wicklund

Scope of Work

This group has an ongoing experimental program at the ZGS centered about the Effective Mass Spectrometer (EMS) and the polarized proton beam. The spectrometer consists of a large aperture magnet and arrays of magnetostrictive-readout wire spark chambers which are used to momentum analyze forward-going charged particles from reactions in a liquid hydrogen or deuterium target. The newly installed $12 \mathrm{GeV} / \mathrm{c}$ beam line utilizes superconducting dipole and quadrupole magnets to transport polarized protons at the highest ZGS energy to the EMS. Recent experiments have included measurements of the polarization parameter in proton-neutron elastic scattering up to $12 \mathrm{GeV} / \mathrm{c}$, and high-statistics studies of spin effects in inelastic reactions, such as $\mathrm{N}^{*}$ and $\Delta$ production.

Earlier, a number of experiments were performed using a highresolution secondary particle beam. More than $5 \times 10^{5}$ events of the type $\pi^{-} p \rightarrow \pi^{-} \pi^{+} n$ have been analyzed for $\rho \omega$ interference, $\pi \pi$ scattering, $K \bar{K}$ threshold effects, etc. Some of the data were taken with a $\pi^{+} n$ initial state, and comparison with the $\pi^{-} p$ data allows a direct determination of the $\rho-\omega$ interference terms. Data were also taken on $\mathrm{K}$ charge exchange and $\mathrm{K}$ production from both proton and neutron targets to allow comparisons with exchange-degenerate Regge models. The $K^{*}$ data are closely related to $\rho \omega$ interference via SU(3). The group is also part of an international collaboration which has constructed and operated a high-precision single-arm spectrometer at FNAL.

Goals and/or Major Results to Date

A high statistics study of $\pi^{-} \mathrm{p} \rightarrow \mathrm{K}^{-} \mathrm{K}^{+} \mathrm{n}(110,000$ events) and $\pi{ }^{+} n \rightarrow K^{-} K^{+} p(50,000$ events) was recently completed at $6 \mathrm{GeV} / \mathrm{c}$. Comparison of the two reactions allow isolation of interference between $\mathrm{K}^{-} \mathrm{K}^{+}$states of differing isospin. The $\mathrm{Y}^{\circ}$ moment shows $f-A_{2}, f^{\prime}-A_{2}^{o}$, and $f-f^{\prime}$ interference effects, and provides the first observation of the $f^{\prime}$ in pion induced reactions. 
ARGONNE NATIONAL LABORATORY

Argonne, Illinois

In the FNAL experiments, the $s$ and $t$ dependence of a variety of reactions such as $\pi p \rightarrow \pi p$ and $\pi p \rightarrow \pi N^{*}$ have been studied with beams of $\pi^{ \pm}, \mathrm{K} \pm, \mathrm{p}$ and $\overline{\mathrm{p}}$ from 50 to $175 \mathrm{GeV} / \mathrm{c}$ on both hydrogen and deuterium targets. A future experiment with this spectrometer will study reactions such as $\pi^{+} \mathrm{p} \rightarrow \mathrm{K}^{+} \Sigma^{+}$.

$* * * * * * * * * * * * * *$

Title of Project: Polarized Target Experiments Group

Person in Charge: A. Yokosawa

Scientific and Professional Staff: P. Auer, D. Hill, K. Nield, B. Sandler, H. Spinka, D. Underwood, Y. Watanabe

Scope of Work

The group is determining the scattering amplitudes in pp elastic scattering at $6 \mathrm{GeV} / \mathrm{c}$. In addition, the energy dependence of some pp (total and elastic) spin correlation parameters from 2 to $6 \mathrm{GeV} / \mathrm{c}$ are being measured. The program is carrled out using the unique polarized proton beam from the ZGS, in conjunction with one of several polarized proton targets. The polarized target consists of $8 \mathrm{~cm}$ frozen glycol, cooled by an $\mathrm{He}^{3}$ cryostat, and can achieve free proton polarizations averaging nearly $90 \%$. A 3000-wire proportional chamber detection system measures the incident and outgoing particle tracks, as well as the recoll proton polarfzation in a carbon polarimeter consisting of four $x-y$ planes and a carbon sheet. Data from the detectors is recorded and processed on-line by an EMR 6050 computer. One of the polarized targets currently in use has a magnet consisting of two superconducting Helmholtz coils arranged in such a way that target polarizations in the (horizontal) scattering plane can be achieved. Also in use is a superconducting solenoidal magnet which is used to rotate the polarization direction of the $6 \mathrm{GeV} / \mathrm{c}$ beam protons by $90 \%$ into the horizontal plane.

This group is also part of a collaboration to measure the polarization parameters in $\pi^{ \pm}, K^{\ddagger}, p$ and $\bar{p}$ elastic scattering from protons from 50 to $200 \mathrm{GeV} / \mathrm{c}$ at FNAL. The experiment has completed its first data run.

Goals and/or Major Results to Date

Recent results have been published on measurements in proton-proton scattering of the two-spin correlation parameter $\mathrm{C}_{\mathrm{NN}}$ as a function of $t$ for energies between 2 and $6 \mathrm{GeV} / c$. Unusually rapid energy variation was observed, which could not be reproduced by current theoretical models. Recently completed were the first measurements 


\section{ARGONNE NATIONAL LABORATORY \\ Argonne, Illinois}

ever made of three-spin correlation parameters in $\mathrm{p}-\mathrm{p}$ elastic scattering; the parameters $(N, S ; 0, S)$ and $(S, N ; 0, S)$ were both measured at $6 \mathrm{GeV} / \mathrm{c}$. Currently, the proton-proton total cross section difference in pure longttudinal spin states $\sigma(\vec{\rightarrow})-\sigma(\overrightarrow{+})$ is being measured between 1.2 and $3 \mathrm{GeV} / \mathrm{c}$.

Future goals include the construction and use of a polarized neutron target, with the objective of separating the nucleon-nucleon elastic scattering amplitudes into their $I=0$ and $I=1$ exchange components.

$$
\star * * * * * * * * * * * *
$$

Title of Project: Hyperon Beta Decay Group

Person In Charge: T. Romanowski

Scientific and Professional Staff: C. Ward, J. Watson

Scope of Work

This group is currently engaged in an experiment to measure the decay asymmetries in $\Sigma^{-} \rightarrow n e^{-} v$, using polarized $\Sigma^{-1}$ 's produced by a $430 \mathrm{MeV} / \mathrm{c} \mathrm{K}$ beam through the reaction $\mathrm{K}^{-} \mathrm{p} \rightarrow \Sigma^{-} \pi^{+}$. Th1s experiment will provide a sensitive test of the Cabibbo model of weak interactions since measured decay asymmetries can be used to determine the sign of the form factor ratio $g_{1} / f_{1}$.

The experiment, which is a collaboration with groups from ohio State and the University of Chicago, is performed using a magnetic spectrometer. The apparatus is composed of an array of proportional wire chambers and conventional wire spark. chambers surrounding a 11quid hydrogen target and placed in a wide-gap C-magnet. The electrons from the $\Sigma^{-}$decay are identified by an array of gas Cerenkov counters of a "crab-eye" design located around the spectrometer. The momentum of the electron and of the final-state $\pi^{+}$are measured in the spectrometer. Data is recorded using an on-line PDP-15 computer.

Goals and/or Major Results to Date

A recent experiment performed by the group with the Argonne Effective Mass Spectrometer measured a number of the polarization parameters in $\Lambda^{\circ}$ production by $6 \mathrm{GeV} / \mathrm{c}$ polarized protons. The experiment was motivated by the possibility of using the polarized proton beam at the ZGS to search for possible polarization effects in hyperon production by polarized protons, since a source of high energy polarized hyperons could be of considerable significance in 


\section{ARGONNE NATIONAI LABORATORY}

Argonne, Illinois

future experiments studying rare hyperon decay modes. The group is planning a future experiment to study the reaction $\mathrm{pp} \rightarrow \Lambda^{\circ} \mathrm{K}^{+} \mathrm{p}$ at 6 and $12 \mathrm{GeV} / \mathrm{c}$, using the polarized beam and the Effective Mass Spectrometer.

$$
\star \star \star \star \star \star \star \star \star \star \star * * * * * *
$$

Title of Project: Theoretical High Energy Physics

Person in Charge: E. Berger

Scientific and Professional Staff: R. Arnold, J. Babcock, R. Cutler, S. Fenster, K. Hidaka, D. Sivers, C, Sorensen, and G. Thomas

\section{Scope of Work}

The group carries out a program of research on a broad spectrum of theoretical and phenomenological topics in strong interactions. Close coordination is maintained with the Argonne experimental groups and users of the ZGS, both in the interpretation of data and in the choice of new investigations. Current research interests of group members include:

1) Analysis of intermediate and high energy polarization data;

2) Hadron Resonance Spectroscopy;

3) Development of theoretical models for exclusive single- and two-pion production reactions;

4) Phenomenological descriptions of high energy two-body, quasitwo-body and multiparticle production processes;

5) Symmetry schcmeo;

6) Models for inelastic diffractive processes; and

7) Neutrino and charm particle phenomenology.

Goals and/or Major Results to Date

Recent results include:

1) Development of a theoretical method to determine the charge conjugation and other resonance parameters of strange axial vec' or mesons via resonance background interference; 
ARGONNE NATIONAL LABORATORY

Argonne, Illinois

2) Explanation of the differences in the momentum transfer distributions between elastic and inelastic diffractive reactions;

3) Topological structure of the Pomeron exchange amplitudes; and

4) Interpretations of data obtained with the ANL-ZGS polarized proton beam.

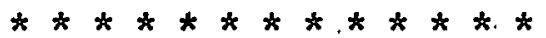

Title of Profect: Bubble Chamber Physics

Person in Charge: M. Derrick

Scientific and Professional Staff: C. Chen, T. Fields; P. Gregory, L. Hyman, K. Jaeger, R. Miller, B. Musgrave, J. Phelan, P.

Schreiner, R. Singer, R. Smith, A. Snyder, and M. Szczekowski

Scope of Work

This group is primarily a bubble chamber physics analysis group, but substantial efforts are also devoted to apparatus work at the ZGS Complex, particularly connected with exploitation of the 12-foot bubble chamber.

The major effort of the group is in neutrino physics. Neutrino exposures of the 12-foot chamber filled with deuterium are being used to make a precise determination of the axial vector form factors. Quasi-elastic scattering $v n \rightarrow \mu^{-} \mathrm{p}$ and also inelastic reactions leading to $\Delta(1238)$ production are studied. By comparing $\pi$ production from neutron and proton targets in the reactions $\nu n \rightarrow \mu^{-} \mathrm{p}^{\circ}, \mu^{-} \mathrm{n} \pi^{+}$ and $\nu p \rightarrow \mu^{-} p \pi^{+}$, the isospin $1 / 2$ and $3 / 2$ constituents of the final state can be separated. The structure of the weak neutral currents is being studied using the reactions $\nu p \rightarrow \nu p, \nu p \rightarrow \nu p \pi^{\circ}, \nu p \rightarrow \nu n \pi^{+}$ and $\nu n \rightarrow \nu p \pi^{-}$. These experiments provide important tests of various theoretical models of the weak neutral current interactions. Neutrino-induced interactions with strange particles in the final state as well as interactions with more than one pion produced are being studied. A second generation experiment using $\gamma$-converting plates in the chamber and with high beam intensitics will start in the fall of 1976. Finally, $\bar{v}$. interactions in the FNAL 15-foot hydrogen bubble chamber are also being analyzed.

Other current experiments in progress are:

1) $K_{T}^{0}$ Decays and Interactions - uses $300 \mathrm{~K}$ pictures taken with a monochromatic $\mathrm{K}_{\mathrm{L}}^{\mathrm{O}}$ beam incident on the 12-foot hydrogen chamber; 


\section{ARGONNE NATIONAL LABORATORY}

Argonne, Illinois

2) $\mathrm{K}^{ \pm}$Nucleon Interactions - a one-million-picture exposure of the 12-foot chamber to $6.5 \mathrm{GeV} / \mathrm{c} \mathrm{K}^{-}$mesons has been made;

3) pp Interactions at $12 \mathrm{GeV}$ - studies of inclusive $\gamma, \pi^{\circ}$ and neutron production are being pursued using a hydrogen-filled track sensitive target surrounded by a $38 \%$ mole hydrogen-neon mixture;

4) pd Interactions at High Energy - pictures have been obtained from an exposure of the deuterium-filled 30-inch chamber at FNAL to $200 \mathrm{GeV}$ protons. Downstream wide gap spark chambers are used to detect and measure the fast, forward-going jet as well as neutral particles originating in the primary. interaction;

5) $\bar{p} p$ and $\bar{p} d$ Interactions at Low Energy - exposures of the 12-foot bubble chamber filled with hydrogen and deuterium have been made to a low energy antiproton beam entering the chamber at about. $700 \mathrm{MeV} / \mathrm{c}$ and coming to rest in the liquid. All of these experiments are being carried out in collaboration with university physicists.

Goals and/or Major Results to Date

Recent achievements include the first identification of a neutrino interaction in liquid hydrogen, the most precise determination to date of the weak axial vector form factor, first observation in a specific reaction channel of weak neutral currents, and establishment of an important $I=3 / 2$ component of the weak neutral current. The major goal of the neutrino experiment in the coming year is to improve the precision of the results obtained so far and to search for new, smaller effects with the second-generation high-intensity neutrino exposure of the 12-foot chamber.

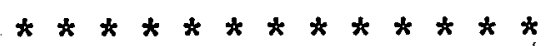

Title of Project: Bubble Chamber Programing Group

Person in Charge: J. Troyer

Scientific and Professional Staff: G. Gieraltowski, P. Rynes, J. Troyer

Scope of Work

This group is a support group which writes, modifies, documents, and maintains computer programs used by the bubble chamber physicists. The group also answers computing-related questions from those physicists, and from others in the High Energy Physics Division. It keeps abreast of new developments and of new acquisitions of computer hardware and software within the Laboratory, and makes sure that this 
ARGONNE NATIONAL LABORATORY

Argonne, Illinois

information is known by those within the Division who have a need for it. The group maintains a program Iibrary of approximately 25 computer programs used in bubble chamber physics. It also corresponds with other laboratories throughout the world in order to share available programs and avoid duplication of effort. In addition to these responsibilities, members of the group serve on computer-oriented and on other divisional committees, and are responsible for communications with the Applied Mathematics Division which operates the central Laboratory computer. Members are also active in the Laboratory's Computer Users' Group.

Goals and/or Major Results to Date

The program which does track matching for tracks measured in different views of bubble chamber photographe hao recently beei extensively modifled to improve the ability of the program to match tracks measured in large bubble chambers having fisheye optics when large numbers of tracks are visible in one picture.

A major project for the near future is the development of a program system which will handle the geometrical reconstruction of tracks in experiments using the Track Sensitive Target in the 12-foot bubble chamber.

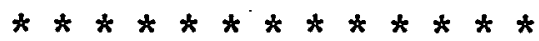

Title of Project: 12-foot Bubble Chamber Development

Person in Charge: K. Jaeger, A. Thomas

Scientific and Professional Staff: K. Jaeger; A. Thomas

Scope of Work

This group provides physics supervision for the 12-foot hydrogen bubble chamber and carries out development work associated with this very large experimental device. The chamber, with a visible volume of about 20,000 liters, is well matched to the special requirements of neutrino experiments, although it is also an excellent detector for strong interaction experiments, being capable of operation with either hydrogen, deuterium or hydrogenneon mixtures.

The most important current area of development involves a track sensitive target (TST). The operation of the chamber with the TST will be a unique resource of the U.S. high energy physics program, and so is expected to be a very important aspect of the future 
ARGONNE NATIONAL LABORATORY

Argonne, Illinois

bubble chamber research program. The advantages of the TST technique can only be fully achieved with a chamber of this size. With the TST filled with hydrogen, it will be possible to study. various final states with multiple neutral particles, many of which will elther interact or convert in the hydrogen-neon mixture surrounding the target.

A second area of development is the installation of tantalum converter plates for future neutrino and antineutrino experiments. The enhanced $₹$-ray conversion probability provided by the plates will significantly enhance the ability to clearly identify neutrino-induced events with one or more produced $\pi^{\circ}$ 's.

Goals and/or Major Results to Date

The first physics run with the TST was completed in December 1975 in a $38 \%$ mole neon mixture. A total of 63,000 physics pictures. were taken while subjecting the target to 2200,000 expansions. The TST, built out of Lexan by CERN, survived the experiment without any mechanical failure. However, the Lexan material employed suffers from large light absorption and thus is of less than desirable optical quality. Future targets will be constructed at ANL of a new Lexan material which has a much lower light absorption. Future experiments will also require running with 70-75\% mole neon mixture and this will require further development of the chamber mechanical systems.

$$
\star \star \star \star \star \star * \star * \star * \star * \star * * *
$$

Title of Project: Automatic Bubble Chamber Film Measuring

Person in Charge: H. Phillips, B. Musgrave

Sclentific and Professional Staff: G. Gieraltowski, R. Smith,

R. Zieman

Scope of Work

A continuing series of POLLY devices have been constructed for automatically measuring bubble chamber $f 11 \mathrm{~m}$, using a computercontrolled light spot to examine the film. The light spot is provided by a cathode-ray tube operating under the control of an XDS Sigma 5 computer. In design of both the hardware and software, particular emphasis has been placed on allowing operator-device interaction, recognizing that difficult situations are most expeditiously solved through operator intervention. The 
POLLY systems have demonstrated unique capabilities for automatically processing data from bubble chamber film in an effective and economical way and POLLY concepts have been widely implemented elsewhere.

POLLY III, the latest in the series, was designed with the large bubble chambers in mind. Systems of this type have been manufactured commercially and acquired by several laboratories. In order to handle film from the large bubble chambers, emphasis in the software has been on measuring the optimum two views for each track rather than the usual three views, followed by immediate on-line geometrical reconstruction with remeasurement of unacceptable tracks.

Goals and/or Major Results to Date

POLLY III has successfully completed several experiments using film from the FNAL 30-inch and the ANL 12-foot chambers. Continued software development is intended to expedite analysis of a large $\mathrm{K}$ p 12-foot chamber experiment. The good quality track display generated by a TV raster scan of a small area on the film will be exploited to enhance operator-machine interaction necessary for complex situations.

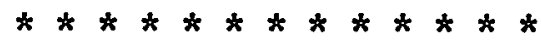

Title of Project: On-Line Computer Development

Person in Charge: H. Phillips

Scientific and Professional Staff: S. Kramer

Scope of Work

Coupling particle detectors directly with computers has greatly reduced the time required for data analysis in many types of high energy physics experiments. In addition, it has made possible real-time monitoring of equipment performance and of the data-taking progress. Development work at Argonne has been directed toward finding the most effective ways to utilize moderately small computers to collect data, analyze it, and to present lumedlate meaningful results to the experimenters.

At present, on-line computing is an essential part of almost all counter experiments at the ZGS. Four Argonne on-1ine systems are available to ZGS users, three EMR $6050^{\prime}$ 's and one XDS Sigma 2. Each 


\section{ARGONNE NATIONAL LABORATORY \\ Argonne, Illinois}

of the on-1ine Argonne EMR machines has $32 \mathrm{~K}$ of core and floating. point hardware. They have proven to be very potent computers for this kind of application. A fourth EMR 6050 has been obtained from ERDA surplus equipment and is available for software development and off-line analysis. A less powerful EMR 6020 has also been obtained from surplus and is used primarily for hardware development. A significant inventory of EMR spare parts has been obtained at minimal cost which will continue to be used to keep the computers in a high state of readiness.

Goals and/or Major Results to Date

These facilities have served the needs of many experimenters well for several years, but the higher data rates which are now achievable make it essential that we continue to develop ways to increase the ZGS on-1ine computing capability. Several hardware improvement projects are being considered for FY 1977, including adding small front-end computers for rapid data-handling from CAMAC interface systems, reserving the $6050^{\prime} \mathrm{s}$ for the computational tasks at which they exce1.

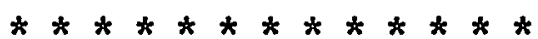

Title of Project: Polarized Target Development

Person in Charge: D. Hill

Scientific and Professional Staff: S. Wang, A. Yokosawa

Scope of Work

During FY 1976, the polarized target PPT-III was used, in conjunction with the superconducting magnet SCM-I, in ZGS experiments on spin amplitudes in pp elastic scattering. In addition to handling the operation of these devices during experimental runs, the polarized target group made improvements to several PPT-III subsystems. Improvements were made to the electrostatic bead-maker which is used to form the target material into the necessary small, frozen droplets. Refinements were made to our process for making vacuum-tight target holders of thin Teflon. The computerized polarization monitor system is being upgraded to accept signals from polarized deuterons in addition to polarized protons, for future experiments. Also, during FY 1976 the helium liquifier system which furnishes liquid helium coolant to the above devices was completely overhauled. 
ARGONNE NATIONAL LABORATORY

Argonne, Illinois

The target PPT-III is now operating with a new superconducting magnet (SCM-II), which has transformed it into the so-called "R and $A$ " target. The current experiment is a measurement of the " $R$ " parameter in pp elastic scattering. The polarized target group contributed in a substantial way to the assembly and testing of this new magnet, and built a new high-stability power supply for it.

In FY 1976 the group also brought into operation a second $\mathrm{He}^{3}-$ cooled target cryostat, called PPT-IV. This is now in use at FNAL in elastic scattering experimente. The group has a major responsibility for the operation and maintenance of this target.

Goals and/or Major Results to Date

In FY 1977, in addition to managing normal operations and maintenance of the above systems, the group will start serious work on a $\mathrm{He}^{3}-\mathrm{He}^{4}$ dilution refrigerator for cooling polarized deuterons. The current cryostats can polarize deuterons no more than $25-30 \%$, whereas better than $40 \%$ is achievable with a dilution refrigerator. As currently envisioned, this refrigerator would take the form of a new lower stage for the PPT-III cryostat, and would be operational in early FY 1978. Some studies of the proper technique for making the deuterated target material will also be carried out.

$* \star * * * * * * * * * * *$

Title of Project: Superconducting Magnet Development

Person in Charge: J. Purcell, R. Niemann

Scientific and Professional Staff: S. Kim, R. Smith, L. Turner, S. Wang

Scope ot Work

Over the past several years, the economy and utility of large DC superconducting magnets have become generally accepted. Much of the progress in this area has resulted from the pioneering development work and operating experience at Argonne. The routine operation of the 12-foot bubble chamber magnet for a period of over 18,000 hours has been the world's first conclusive demonstration of the general practicality of large-scale superconducting magnets, Subsequent achievements include the construction of the magnets for the FNAL 15-foot bubble chamber, and the construction of magnets for high energy physics devices. The application of 


\section{ARGONNE NATIONAL LABORATORY}

Argonne, Illinois

superconducting magnets to energy storage and generation devices has recently become of great importance to the national energy program, and the magnet group is actively working on magnets for such purposes, as well as for high energy physics applications.

Two of the more important areas of application supported by funding from sources outside high energy physics are:

1) A large dipole magnet, for magnetohydrodynamic (MHD) power generation is under construction. It will be used at the U-25 MHD facility in Moscow, as part of an exchange agreement between the USA and USSR. Completion and shipment of the magnet system is scheduled for FY 1977.

2) Considerable effort is being devoted to studies of superconducting magnet systems in controlled thermonuclear research (CTR), especially to the design of toroidal field coils and poloidal field coils for a Tokamak Experimental Power Reactor (TEPR). Experimentally, it is hoped to begin studies of pulsed superconducting magnet systems, such as would be required for future CTR poloidal coils, in FY 1977.

Goals and/or Major Results to Date

The past year has seen the completion and installation of the world's first completely superconducting beam line system. The beam line, which transports $12 \mathrm{GeV} / \mathrm{c}$ polarized beams to the Effective Mass Spectrometer, consists of ten dipole and two quadrupole magnets, installed in four cryostats. These random-wound magnets, originally designed for the Superconducting Stretcher Ring (SSR), provide beam-line-quality field precision with simple design and construction techniques.

Two other important projects were completed during FY 1976:

1) The superconducting magnet for the novel "A and $R$ " polarized target was constructed, and tested, and is in operation. This is the second superconducting magnet built at Argonne for polarized target experiments.

2) A long superconducting solenoid magnet, designed to rotate the polarization of the $6 \mathrm{GeV} / \mathrm{c}$ ZGS polarized proton beam by $90^{\circ}$ about the beam direction, was completed. The magnet system was designed at ANL, with the magnet itself being purchased from a commercial vendor. 
ARGONNE NATIONAL LABORATORY

Argonne, Illinois

Title of Project: $500 \mathrm{MeV}$ Booster Synchrotron

Person in Charge: R. Martin, J. Simpson

Scientific and Professional Staff: E. Crosbie, M. Foss, T. Khoe, R. Timm, T. Hardek, G. Volk, C. Potts, W. Praeg, D. Suddeth, D. Schmitt, J. Bywater, J. Peerson, K. Thompson

Scope of Work

This group is engaged in the design and construction of a small, rapid cycling, $500 \mathrm{MeV}$ booster synchrotron to be used in conjunction with the Zero Gradient Synchrotron and for use as a source of protons for a spallation neutron source. By using a novel injection scheme in which $\mathrm{H}^{-}$ions are stripped of their electrons after injection on an equilibrium orbit, proton beam intensities normally unattainable in such a small machine will be realized.

This high intensity beam, when injected into the ZGS at $500 \mathrm{MeV}$, will permit the ZGS intensity to increase several-fold.

Efforts are directed toward lattice, magnet, $\mathrm{rf}$, fast kicker, vacuum, diagnostic, control and ancillary system design and construction.

Goals and/or Major Results to Date

The design goal of the machine is $5 \times 10^{12}$ protons per pulse accelerated to $500 \mathrm{MeV}$ at a $30 \mathrm{~Hz}$ repetition rate. Eight such pulses will be transferred to the ZGS each ZGS cycle. During the interval between ZGS cycles ( $\sim$ every $4 \mathrm{sec}$ ), $500 \mathrm{MeV}$ protons will be available for the production of intense, pulsed neutron beams to be used in research in the physics of condensed matter.

Design and construction of the synchrotron and its supportive systems is well underway. Initial beam tests in the booster are scheduled to begin early in calendar 1977, with beam transfer to the ZGS to be attempted the following fall.

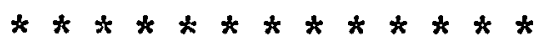

Title of Project: ZGS and Experimental Facilities Operation

Person in Charge: R. Martin, R. Kustom, E. Parker, J. Simpson

Scientific and Professional Staff: R. Bouie, F. Brumwell, J. Dawson, K. Jaeger, R. Klem, M. Knott, R. Moffett, C. Potts, A. Thomas, R. Timm, J. Watson. 


\section{ARGONNE NATIONAL LABORATORY \\ Argonne, Illinois}

Scope of Work

This group operates the ZGS injector and main ring accelerator, the experimental areas including the primary and secondary beam Iines, and the 12-foot bubble chamber. Maintenance and development of the ion sources, preaccelerator, $50 \mathrm{MeV}$ linear accelerator, main ring magnets and vacuum system, computers and beam control devices, primary and secondary beam lines, beam control magnets, beam diagnostics, and the 12-foot bubble chamber are all done with minimum interference with scheduled high energy physics activities. Major improvements carried on by this group are the installation of a simultaneous resonant extraction system and implementation of the unique polarized proton facility. The group applies effort to related activites, such as, Booster II design, construction, installation and testing, and IPNS planning. The ZGS experimental area has two primary proton beams of $12 \mathrm{GeV} / \mathrm{c}$, twelve secondary beams of various energy and momenta and two low intensity test beams. Approximately three to four new secondary beam configurations or modifications are undertaken annually. The bubble chamber has averaged 50,000 to 60,000 experimental pictures per operating week over the past five years. From a total of more than 5,000,000 pictures, one million have been neutrino exposures. The chamber has operated with a track sensitive target in a $35 \%$ mole neon hydrogen mixture.

Goals and/or Major Results to Date

There has been a three-fold increase in beam intensity during the last three years due to improvements in acceleration and extraction. The goal is another factor of three with implementation of $\mathrm{H}^{-}$and Booster II injection. The polarized beam has increased by a factor of one hundred to $2 \times 10^{10} \mathrm{ppp}$ over the same period. The goal is $1 \times 10^{11}$ ppp. A superconducting beam line including ten dipoles and two quadrupoles hàs been installed. An average of eighteen high energy physics experiments completed per year will be maintained. Two miliion pictures will be taken in the 12-foot bubble chamber in deuterium for neutrinos and antineutrinos and 500,000 will be taken in the TST.

$\star \star \star * \star * \star * \star * * * * * * *$

Title of Project: Accelerator Research and Development

Person in Charge: R. Martin, E. Parker, J. Simpson

Scientific and Professional Staff: R. Arnold, Y. Cho, E. Crosbie, J. Fasolo, M. Foss, T. Khoe, R. Lari, J. Moenich, R. Moffett, L. Ratner 
ARGONNE NATIONAL LABORATORY

Argonne, Illinois

Scope of Work

In addition to their routine involvement in the ZGS improvements, efforts of this group also include developments of a high current $\mathrm{H}^{-}$ion source, a high energy polarized proton beam facility, a medical $240 \mathrm{MeV}$ proton synchrotron, a proton radiograph facility, a $50 \mathrm{MeV}$ storage ring ion beam fusion, and an intense pulsed neutron source (IPNS) facility. The high current $\mathrm{H}^{-}$ion source is to employ a high intensity positive ion source in conjunction with a sodium vapor charge-exchange cell. The high energy polarized beam provides the study of the proton as a spin $1 / 2$ particle in basic nucleon-nucleon interactions. The ZGS is the only high energy proton synchrotron with a magnet lattice that will allow the acceleration of polarized protons without serious depolarization. A proton synchrotron of $240 \mathrm{MeV}$ has been designed for proton radingraphy for early detection of cancer. In it, the extraction of a high quality proton beam occurs by stripping the electrons from the $\mathrm{H}^{-}$ions in very thin metal foils. The proton radiography facility provides study of techniques of making proton radlographs and presenting the information. The $50 \mathrm{MeV}$ storage ring is currently under design to study advanced accelerator concepts. Studies of an accelerator system capable of igniting the fusion reaction in small pellets of deuterium tritium are being. carrfed out. The IPNS facility would permit unique information about the structure and dynamics of materials.

Goals and/or Major Results to Date

The objective of the $\mathrm{H}^{-}$ion source program is to obtain a 10 to 15 $\mathrm{mA}$ full energy $\mathrm{H}^{-}$beam. It is intended to increase the polarized proton beam to $1 \times 10^{11}$ protons per pulse and to make External Proton Beam I dichromatic. A proton diagnostic accelerator and proton radiography facility would be constructed and operated at Argonne as a National Proton Radiographic Facility for clinical trials. The $50 \mathrm{MeV}$ storage ring studies would include electron cooling and accumulator ring behavior. An ion beam fusion reactor demonstration facility would be constructed. The goal would be to eventually produce useful energy output. The goal of the IPNS activity is the design, construction, and operation of larger pulsed neutron facility.

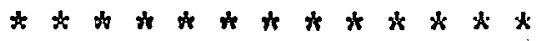


BROOKHAVEN NATIONAL LABORATORY

Upton, New York

Contract No. EY-76-C-02-0016

Title of Project: FNAL Experiments 非48,E435, E436; AGS \#676

Person in Charge: L.B. Leipuner

Scientific and Professional Staff: W.M. Morse, R.C. Larsen

Scope of Work

A rather unexpected result from the CERN ISR was that the production of prompt leptons (electrons and muons) at these high energies and at high transverse momenta was quite large, about one lepton per 10000 pions. A reexamination of the data from an experiment we had done at the AGS on muons for another purpose revealed a similar result. Our results had been at low transverse momenta and at low energy, $\leq 28 \mathrm{GeV}$. For the last several years we have been examining this phenomena at Fermilab.

The great interest in this phenomena is due to the possibility that these prompt leptons are the decay signature of the very interesting $W$ particle, the intermediate boson, or of charmed mesons. If this were the case one would be able to see these effects by measuring the polarization of the muons. The possible tendency of these particles to come in pairs rather than to be singly produced also bears on the question. The ratio of positive to negative leptons as well as their energy distribution is also illuminating.

At this time we are preparing a series of experiments, to be done at the Brookhaven AGS, along these lines. The lower energies of this machine allow a more careful examination of the detailed behavior of these muons.

Goals and/or Major Results to Date

To date we have confjrmed that the ratio of prompt muons to pions is about one part in $10^{4}$ at zero transverse momentum and 300 to $400 \mathrm{GeV}$. Certainly to within $20 \%$ all these muons are produced in pairs: The pairs seem to consist of 1 positive and 1 negative muon. The invariant mass of the pair seems to be about $900 \mathrm{MeV} / \mathrm{c}^{2}$. The polarization of the positive muons is zero indicating an electromagnetic type origin for these pairs. The polarization is zero at a transverse momentum of $2 \mathrm{GeV} / \mathrm{c}$ as well as at $0 \mathrm{GeV} / \mathrm{c}$. 


\section{BROOKHAVEN NAT IONAL LABORATORY \\ Upton, New York}

At the AGS we will study the production of these pairs as a function of energy and atomic number. We will also investigate the invariant mass distribution of the parent particle or state of these muons.

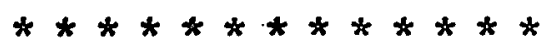

Title of Project: AGS Experiments $589 \& 639$

Person in Charge: George Kalbfleisch

Scientific and Professional Staff: G.R. Kalbfleisch, R.C. Strand, $\mathrm{J}$. Alspector

Scope of Work

This is a collaboration with a Columbia University group to study neutrino interactions in the neon-filled 7 foot Bubble Chamber at Brookhaven. This will utilize the narrow band horn-focused neutrino beam to get a good definition of the neutrino energy. We hope to untangle the vector and axial vector nature of the weak neutral current by studying the energy distribution in the final state. The neon will serve as a calorimeter to convert neutrals and the defined initial energy is essential for this disentanglement. We will also study strange and charmed particle production in neutrino interactions. We also hope to look for neutrino-electron interactions in a wide-band run.

As part of this experiment we will measure the incident neutrino flux with a spectrometer in the decay space to measure the parent hadrons.

\section{Goals and/or Major Results to Date}

The narrow band beam has been tested successfully by measuring muon fluxes at various depths in the hadron shield with existing ionization chambers and comparing with predictions. We have also built and tested the hadron spectrometer necessary for measuring the incident flux.

$$
* * * * * * * * * * * * * *
$$

Title of Project: AGS Experiments $632 \& 687$

Person in Charge: George Kalbfleisch

Scientific and Professional Staff: G.R. Kalbfleisch, R.C. Strand, J. Alspector, J. Scharenguivel 
BROOKHAVEN NATIONAL LABORATORY

Upton, New York

Scope of Work

These experiments are in collaboration with a group from the University of Rochester. Experiment 632 is a study of deep inelastic muon scattering from nuclear targets. One aim is to determine the A-dependence of the cross section at low $x$ in order to study shadowing of virtual photons by nuclear matter. Another is to study production of muon tridents as a test of the statistics of the muon. We also study multi-muon final states to look for new phenomena such as heavy leptons decaying to muons.

Experiment 687 is to study dimuon production from 16 and $24 \mathrm{GeV}$ pions on heavy nuclei. The purpose here is to understand the $x$ and $P_{\perp}$ dependence of $\mathrm{J}$ production especially near threshold where one might see an important difference between production from pions and that from protons. We will also measure the dimuon spectrum at all masses. It may also be possible to study single muon production and see how many of the single muons come from the measured dimuon spectrum.

\section{Goals and/or Major Results to Date}

Experiment 632 has finished data taking and work is proceeding on reconstructing events. We are close to being able to make a first pass through the data and extract some preliminary physics results.

Experiment 687 is now in the construction phase. We are building additional particle detection equipment and are rearranging the geometry of Experiment 632 in order to be ready for a data run in November 1976.

$$
* * * * * * * * * * * * *
$$

Title of Project: Fermilab Experiment 254

Person in Charge: George Kalbfleisch

Scientific and Professional Staff: G. Kalbfleisch, J. Alspector

Scope of Work

The purpose of this experiment is to see if the neutrino from $\pi$ decay is different from the neutrino from $K$ decay. This was done in collaboration with the Caltech group using their existing neutrino facility at Fermilab. The dichromatic beam provides $K$ neutrinos near the full hadron beam energy and $\pi$ neutrinos at about $40 \%$ of the full energy. We search for a difference in the neutrinos by measuring their cross 


\section{BROOKHAVEN NATIONAL LABORATORY \\ Upton, New York}

sections at the same neutrino energy. This necessitates runs at two different hadron beam energies to make the neutrino energies overlap.

As an addition to the existing equipment we installed a muon monitor at the end of the hadron decay space. This allows us to continuously monitor the muon $f l u x$ and, by means of computer simulation, derive the neutrino flux. Along with primary and secondary beam monitors, this reduces the systematic errors in the cross section measurement.

We have also installed time-of-flight circuitry to compare event times with accelerator RF phase. This allows us to measure the neutrino velocity.

\section{Goals and/or Major Results to Date}

We have published the results of the first direct measurement of the neutrino velocity. Within the accuracy of the measurement it is the speed of light. We have compared measured distributions from the muon monitor with computer predictions and find good agreement. Along with the rest of our collaboration we are analyzing the neutrino events and hope to have a preliminary comparison of neutrino cross sections in a few months. We also hope to have a statement soon about the production of a certain class of neutral heavy leptons, based on time-of-flight information.

$$
* \star * * * * * * * * * * * *
$$

Title of Project: ISR Experiment 806 (New Group)

Person in Charge: R.B. Palmer (BNL); W.J. Willis (CERN)

Scientific and Professional Staff: J. Cobb, R. Hogue, R. Palmer,
D. Rahm, P. Rehak, I. Stumer

Srnpe of Work

The primary object of the experiment is to observe and study high. effective mass electron pairs. Apparatus has been constructed to identify and measure electrons and photons produced in lile collision of protons on protons in the Intersecting storage Ring at CERN, Geneva, Switzerland. The apparatus surrounds the intersection region and covers a solid angle of approximately 4 steradians. Energy measurement of both electrons and photons is provided by a calorimeter consisting of spaced $1 \mathrm{~mm}$ thick lead plates in liquid

- argon. Ionization in the liquid argon produces electric charge 
which is picked up on the plates and, when summed, gives the particle energy. Electron identification is provided by the observation of transition radiation produced by the electrons transversing 1200 lithium foils and measured by proportional chambers. In addition scintillation counters and further planes of proportional chambers are provided to define the track directions and ionization.

Other objects include the study of low mass electron pairs, single electron and photon production, high transverse momentum $\pi^{\circ}$ production and the study of jets.

Goals and/or Major Results to Date

Preliminary results include the observation of $\mathrm{J}$ particles decaying into two electrons and higher mass electron pairs up to the order of $9 \mathrm{GeV}$. When calibrated such data will be compared with parton model and other scaling predictions.

$$
* * * * * * * * * * * * *
$$

Title of Project: FNAL E-268

Person in Charge: Howard A. Gordon

Scientific and Professional Staff: H.A. Gordon, G.J. Donaldson, Kwan-Wu Lai

Scope of Work

With groups from $\mathrm{Caltech}$ and $\mathrm{LBL}$ we have been measuring inclusive photon production at large, transverse momentum from $\pi^{+}, \mathrm{K}^{ \pm}$and $\mathrm{p}(\overline{\mathrm{p}})$ beams at 100,200 , and $300 \mathrm{GeV} / \mathrm{c}$. This data comes from lead scintillator sandwich hodoscope of 70 horizontal and 70 vertical counters. The energy and position of all photon showers in the hodoscope have been found. Specifically, we have data on single photons, two photons ( $\pi^{\circ}$ and $\eta$ ), and three photons $\left(\omega^{\circ}\right)$ for $\mathrm{p}_{1}>1 \mathrm{GeV} / \mathrm{c}$ and for $-0.1<\mathrm{x} / /<1$ from a hydrogen target. Another phase of this experiment was to search for a high mass $\mathrm{J}^{\mathrm{P}}=0^{-}$state composed of $c \bar{c}$ quarks produced peripherally in $\pi^{-} \mathrm{N}$ interactions. As a byproduct of this search, substantial data was collected on the react lons $\left.\left.\pi^{-} N \rightarrow n^{\prime}(958) N ; \pi^{-}{ }^{-} \omega_{N} ; \pi^{-} p \rightarrow \pi^{\circ} \pi^{\circ}\left(f^{\circ}(] 260\right)\right), h^{0}(2000)\right)$; and $\pi \mathrm{N} \rightarrow \mathrm{nnN}$.

Goals and/or Major Results to Date

G. Donaldson, et a1., Phys. Rev. Lett. 36,1110 (1976) reports that the ratio of invariant cross sections $\sigma\left(\mathrm{pp} \rightarrow \pi^{\mathrm{OX}} / \sigma(\pi \mathrm{p} \rightarrow \pi \mathrm{X})\right.$ decreases with increasing $p_{\perp}$ and is independent of energy when expressed as a 
function of $x_{R}=p_{\pi o} / p_{\max }$. This scaling can be understood as a : difference in the internal structure of the $\pi$ compared to the proton. These results were for $\theta_{\mathrm{cm}}=90^{\circ}$ but soon we shall have data for $x / / \rightarrow 1$. We have also reported the ratio of inclusive $\eta$ production to that of $\pi^{0}$ 's is $\sim 1 / 2$ for $p_{1} \gtrsim 2 \mathrm{GeV} / \mathrm{c}$ independent of $p_{1}$, particle type and beam momentum. We hope soon to have results on single (direct) photon production and inclusive $w$ production. In the other phase of the experiment we have an upper limit of $\sim 1 / 2 \mathrm{nb}$ for $\sigma . \mathrm{B}$ for a pseudo scalar with mass $\sim 3 \mathrm{GeV}$. We hope to complete the analysis of the $\pi^{\circ} \pi^{\circ}$ and $\eta \eta$ events.

$$
* * * * * * * * * * * * * *
$$

Title of Project: AGS 686

Person in Charge: Howard A. Gordon

Scientific and Professional Staff: H.A. Gordon, G.J. Donaldson, $\bar{R}$. B. Palmer

\section{Scope of Work}

This is an approved experiment to search for associated production of a charmed meson and a charmed baryon in the reaction $\pi^{-} p \rightarrow D^{-} C^{+}$ utilizing the MPS in the HEUB. It is a collaboration between BNL, Ũniversity of Pennsylvania, Virginia Polytechnic. Institute, Brandeis University, Syracuse University and the University of Cincinnati. This rare reaction will be triggered by selecting a single $\mathrm{e}^{-}$with no accompanying photons or $\mathrm{e}^{+}$. In this way there will be large acceptance for associated charm production with a very high rejection of normal hadronic events. The trigger is achieved by employing two 1ithium foil transition radiation detectors (TRD) and a leadscintillator shower detector (SD). A second important objective of this experiment is to investigate completely in the MPS those events which have a single electron. This should shed light on the nature of the direct lepton production.

Goals and/or Major Results to Date

We are currently building the TRD' $\varepsilon$ and SD. We hupe to take duta in 1977. 


\section{BROOKHAVEN NATIONAL LABORATORY \\ Upton, New York}

Title of Project:' AGS Experiment 非82

Person in Charge: S.U. Chung (BNL); J.R. Bensinger (Brandeis)

Scientific and Professional Staff: A. Etkin, S.U. Chung, J. Kopp,

D.N. Michael, S.D. Protopopescu; K. Foley, H. Goldman, S.J.

Lindenbaum, T.W. Morris, S. Ozaki, E. Platner, A. Saulys,

D. Wheeler, E. Willen, W. Love (BNL); also collaborators from

Brandeis U., C.C.N.Y., U. of Massachusetts, Southern Mass. U.

Scope of Work

Using the BNL Multiparticle Spectrometer, the major aim of the experiment is to search for the associated charmed-particle production with a $\pi^{-}$beam at $22-24 \mathrm{GeV} / \mathrm{c}$. The trigger relies on detection of a $\mathrm{K}^{+}$and a proton at a point in phase space where the ordinary non-charm interaction is expected to be small but the charm production should be relatively high. The waterCerenkov counter of 非557, an existing gas Cerenkov counter and a high-pressure Cerenkov counter (being built) will be utilized in the experiment. The trigger will be accomplished via a randomaccess-memory coincidence-matrix system, which is under construction.

Goals and/or Major Results to Date

A target-region basket containing 10 capacitive-readout spark chamber modules and 3 proportional wire chambers has been constructed.

$$
\star * * * * * * * * * * * * * *
$$

Title of Project:. AGS Experiment 非557

Person in Charge: S.U. Chung (BNL); J.R. Bensinger (Brandeis)

Scientific and Professionàl Staff: S.U. Chung, J. Kopp, D.N. Michael, S.D. Protopopescu, K. Foley, H. Goldman, S.J. Lindenb aum, T.W. Morris, S. Ozaki, E. Platner, A. Saulys, D. Wheeler, E. Willen (BNL); also collaborators from Brandeis U., CCNY, U. of Massachusetts, U. of Pennsylvania.

\section{Scope of Work}

The aim of the experiment was to explore high-mass $\mathrm{K}^{+}$states in the reaction $\mathrm{K}^{-} \mathrm{p} \rightarrow \mathrm{K}^{-} \pi^{+} \mathrm{n}$ at $6 \mathrm{GeV} / \mathrm{c}$ with BNL Multiparticle Spectrometer. An array of capacitive-readout spark chambers was constructed to 


\section{BROOKHAVEN NATIONAL LABORATORY \\ Upton, New York}

detect wide-angle two-prong events. In addition, a water threshold Cerenkov counter was set up to identify the recoil pions:

Goals and/or Major Results to Date

The whole data sample has been completely processed, and shows a $6 \sigma$ enhancement at mass $1780 \mathrm{MeV}$. Moment analysis of the $\mathrm{K}^{-} \pi^{+}$ spectrum is currently in progress.

$$
* * * * * * * * * * * * *
$$

Title of Project: AGS Experiment 非 673

Person in Charge: S.U. Chung

Scientific and Professional Staff: S.U. Chung, D.N. Michael, S.D. Protopopescu (BNL); also collaborators from Florida state U.

\section{Scope of Work}

This experiment is a search for cascade resonances in the reaction $\mathrm{K}^{-} \mathrm{p} \rightarrow \mathrm{K}^{+}+\mathrm{X}^{-}$at $4-5 \mathrm{GeV} / \mathrm{c}$ with the use of the BNL Multiparticle Spectrometer. It employs a $\mathrm{K}^{+}$detector sensitive to $\mathrm{K}^{+}$momentum in the range 200 to $600 \mathrm{MeV} / \mathrm{c}$. The detector consists of a sandwich of 8 brass plates to stop the $\mathrm{K}^{+}$and 9 scintillator planes to detect the signals from a stopping $\mathrm{K}^{+}$as well as from decay products. This detector, in addition to an existing $\mathrm{K}^{+}$detector of the same design, will be used in the trigger.

Goals and/or Major Results to Date

Construction of the $\mathrm{K}^{+}$detector is currently in progress.

$$
* * * * * * * * * * * \dot{x} * *
$$

Title of Project: Measurement of Polarization in $\mathrm{K}^{+} \mathrm{n} \rightarrow \mathrm{k}^{+} \mathrm{n}$; AGS Experiment \#641.

Person in Charge: M. Sakitt

Scientific and Professional Staff: M. Sakitt, J. Skelly, G. Snape, A. Stevens. 
BROOKHAVEN NATIONAL LABORATORY

Upton, New York

Scope of Work

In order to resolve some of the ambiguities in the low energy $I=0, K N$ phase shift analysis, we have designed an experiment to measure the polarization of the recoil neutron in the reaction $K^{+} n \rightarrow K^{+} n$. If one compares phase shift solutions that contain exotic resonances with those that do not, one finds that the recoil neutron polarization flips sign, but is always rather large, .8 . Our experiment will measure this polarization through the momentum region of the possible resonance. We utilize the fact that in the resonance region the recoil neutron has a momentum such that the $\mathrm{np} \rightarrow \mathrm{np}$ reaction is a good analyzer of the neutron polarization. The experiment is a spark chamber - counter experiment sitting in the hiflux low energy beam (C4) at the AGS.

Goals and/or Major Results to Date

A11 the equipment has been built and was tested during an initial setup run last spring. During the coming fiscal year we expect to be taking data and doing some preliminary analysis.

$$
* * * * * * * * * * * * * *
$$

Title of Project: Study of $\mathrm{K}^{+} \mathrm{d}$ Interactions, AGS Experiment $\$ 499$

Person in Charge: M. Sakitt

Scientific and Professional Staff: M. Sakitt and J. Skelly

Scope of Work

We are analyzing an exposure of $\mathrm{K}^{+}$mesons incident on the 30 inch deuterium bubble chamber in the momentum region below $1 \mathrm{GeV} / \mathrm{c}$. The reactions being studied are $\mathrm{K}^{+} \mathrm{d} \rightarrow \mathrm{K}^{+} \mathrm{d}, \mathrm{K}^{+} \mathrm{d} \rightarrow \mathrm{K}^{\circ} \mathrm{pp}, \mathrm{K}^{+} \mathrm{d} \rightarrow \mathrm{K}^{+} \mathrm{np}$ in an effort to understand the low energy $I=0, K N$ system. Measurements of the different cross sections, as a function of incident $\mathrm{K}^{+}$ momentum will aid in improving our knowledge of the phase shift solutions. A compilation of the known data has also been underway so that a more complete phase shift analysis can be accomplished. Interest in these results is motivated by the possible existence of an exotic positive-strangeness, isospin zero baryon resonance.

Goals and/or Major Results to Date

We have finished our study of the $\mathrm{K}^{+} \mathrm{d}$ elastic reaction, where in addition to the different cross sections, we have extracted the ratio of the real to imaginary parts of the forward scattering amplitude. 


\section{BROOKHAVEN NATIONAL LABORATORY}

Upton, New York

The final results of the differential cross sections for the reactions $\mathrm{K}^{+} \mathrm{n} \rightarrow \mathrm{K}^{\circ} \mathrm{p}$ and $\mathrm{K}^{+} \mathrm{n} \Rightarrow \mathrm{K}^{+} \mathrm{n}$ should be in hand during the early part of the coming fisçal year.

$$
* * * * * * * * * * * * * *
$$

Title of Project: Study of $\mathrm{K}_{\mathrm{L}}^{\mathrm{o}}$ Decays, SLAC Experiment 非8

Person in Charge: M. Sakitt

Scientific and Professional Staff: M. Sakitt, G. Snape, A. Stevens

Scope of Work

We are engaged in a study of the dominant decays modes of the $\mathrm{K}_{\mathrm{L}}^{\mathrm{O}}$ meson $\mathrm{K}_{\mathrm{L}}^{\mathrm{O}} \rightarrow \pi^{+} \pi^{-} \pi^{0}, \mathrm{~K}_{\mathrm{L}}^{0} \rightarrow \pi \mathrm{e} \nu, \mathrm{K}_{\mathrm{L}}^{\mathrm{O}} \rightarrow \pi \mu \nu$. In collaboration with the SLAC streamer chamber group we have carried out a large run in the 2 meter chamber which yielded approximately $2 \times 10^{6}$ pictures. In addition to having the optical information of the decay tracks, we measured the time of flight of the $\mathrm{K}^{\circ}$ and therefore have a kinematically constrained system. This experiment has a significantly higher detection efficiency than previous experiments and yields good separation among the various modes. Our study of the $\mathrm{K}^{\mathrm{O}} \rightarrow \pi^{+} \pi^{-} \pi^{\mathrm{O}}$ mode is yielding an accurate knowledge of the matrix's dependence on the energy of the neutral pion. The study of the $\mathrm{K}^{\mathrm{O}} \rightarrow \pi \mathrm{e} \nu$ mode is both yielding a measurement of the $f_{+}\left(q^{2}\right)$ form factor and providing limits on possible scalar and tensor impurities in the basic weak interaction. The study of the $\mathrm{K}^{\circ} \rightarrow$ mL mode will provide a measurement of both $f_{+}\left(q^{2}\right)$ and $f_{o}\left(q^{2}\right)$ and thereby a measurement of the divergence of the vector current, and provide an estimate of the degree of $S U(3)$ breaking that occurs in the scmi-leptonic $K$ decays.

Goals and/or Major Results to Date

Preliminary results on the decay mode $\mathrm{K}^{\mathrm{O}} \rightarrow \pi^{+} \pi^{-} \pi^{\circ}$ have yielded a result for the slope parameter $\mathrm{g}$ of $\mathrm{g}=0.645 \pm .026$, where the error includes both statistical and systematic effects. We expect this error to decrease in our final results. Our detection efficiency was $98 \%$ across the entire Dalitz plot, the highest efficiency experiment to date.

Our preliminary result for the decay mode $\mathrm{K}^{\mathrm{O}} \rightarrow$ me $\nu$ has yielded a result for the $q^{2}$ dependence of the $f_{+}$form factor. Again this error includes both statistical and systematic effects and should decrease in our final result.

$$
* * * * * * * * * * * * * *
$$




\section{BROOKHAVEN NATIONAL LABORATORY \\ Upton, New York}

Title of Project: AGS Experiments $\# 631$ and $\# 671$

Person in Charge: T.F. Kycia

Scientific and Professional Staff: I-H. Chiang, T.F. Kycia, K.K. Li, M.D. Marx, L.S. Littenberg

Scope of Work

In the past, the group has been conducting experiments on rare $\mathrm{K}$ meson decay and total cross section measurements. In AGS experiment \#631 just completed, a proportional chamber spectrometer, a hydrogenfilled Cerenkov counter and a lead glass Cerenkov counter array were used to study radiative decays of $\mathrm{K}^{\circ} \mathrm{L}$ mesons at the AGS. In the next experiment, to study direct lepton production, almost all the equipment from the previous experiment will be reutilized. It will be set up in a high energy charged particle beam at the AGS. A "target region" system, consisting of a liquid hydrogen target, lead-scintillation counter sandwiches, additional proportional chambers and two layers of lead glass Cerenkov counters, is being assembled. The combination of all that equipment will provide a large solid angle system with which most of the particles originating in the target will be detected. Events with electron pairs or single electrons will be studied. Data will be taken for different incident particles and at different momenta.

Goals and/or Major Results to Date

$25 \%$ of the data on $\mathrm{K}^{\circ}$ radiative decay have been analyzed. Several hundred $\mathrm{K}^{\mathrm{O}} \mathrm{L} \rightarrow \pi^{+} \pi^{-} \gamma$ decays have been observed, to be compared with a total of only 23 events observed previously. The matrix element for that decay was found to be consistent with a CP-conserving magnetic dipole transition. The first example of the decay $\mathrm{K}_{\mathrm{L}}^{\mathrm{O}} \Rightarrow \mathrm{e}^{+} \mathrm{e}^{-} \mathrm{Y}$ was observed where one event was expected on the basis of theoretical predictions.

The observed direct lepton production $c$ an be due to a combination of vector meson decay, the Dre11-Yan process and charm particle decay. Electron pair production will be studied in the previously unobserved low mass range down to $250 \mathrm{MeV}$. There is a good possibility that charm particle pair production may be observed.

$$
* * * * * * * * * * * * * *
$$


BROOKHAVEN NATIONAL LABORATORY

Upton, New York

Title of Project: FNAL Experiment 非104.

Person in Charge: T.F. Kycia

Scientific and Professional Staff: I-H. Chiang, T.F. Kycia, K.K. Li, $\because \cdots \quad$ M.D. Marx, L.S. Littenberg

Scope of Work

The method of measuring total cross sections was refined and used at the AGS to observe many new resonances. These techniques are being adopted at Fermilab to study total cross sectinns nf different charged particles on protons and deuterons as a function of energy. Measurements have been carried out to a maximum momentum of $280 \mathrm{GeV} / \mathrm{c}$. With the upgrading of the beam line to $375 \mathrm{GeV} / \mathrm{c}$ one final set of measurements at the higher momenta will be taken during the early part of 1977 .

Goals and/or Major Results to Date

Total cross sections of $\pi$ mesons $K$ mesons and protons on protons and deuterons have been observed to rise with increasing energy at Fermilab. In the next run, the total cross section measurements will be extended by another $100 \mathrm{GeV} / \mathrm{c}$ and with that increase, it may be possible to observe the total cross section of antiprotons to also rise.

$$
* * * * * * * * * * * * *
$$

Title of Project: Physics Spectrometer Group, AGS Experiment \#679

Person in Charge: S. Ozaki, S.J. Lindenbaum

Scientific and Professional Staff: K.J. Foley, J.H. Goldman, W.A. Love, E.D. Platner (Physics Department), A. Etkin, T.W. Morris, A.C. Saulys, C.D. Wheeler, E.H. Willen (Accelerator Department)

Scope of Work

While this group of physicists, which represents both Physice and Accelerator Departments, is responsible for the technical operation, maintenance and improvement of the MPS facility, they also carry out a particle physics research program with it. This group, in collaboration with a CCNY group, has an approved experiment, 非679, in which the production of high mass $\mathrm{K}^{*}$ and $\mathrm{K}^{*}-1$ lke objects will be studied. This experiment will be run using the new HEUB to the 
MPS. Here the physics of interest is, in addition to the study of high mass $\mathrm{K}^{*_{1}} \mathrm{~s}$, a search of charmed mesons, which are expected to decay like $\mathrm{K}^{*}$, and charmed baryons which are expected to behave like heavy hyperons, particularly produced in association. The MPS will be triggered with high momentum forward $\mathrm{K}^{+}$in conjunction with high overall multiplicity. For this purpose and for many of the future experiments at MPS, a new sophisticated trigger logic "RAM" is being developed in this group. Our standard $\mathrm{V}^{\circ}+n \pi$ trigger will also be used for the experiment.

In preparation for this experiment and other "charm" oriented experiments where production cross section is very smal1, an MPS beam rate up-grade program is in progress. One phase of it is to expand the spark chamber readout capability from 8 sparks per readout to 16 : sparks for all MPS chambers and the other, is to supplement the beam region of. the spark chambers with PWC's.

\section{Goals and/or Major Results to Date}

The rate upgrading is expected to take place during this fall and next spring in conjunction with this experiment. We plan to have a unit of the RAM trigger system ready in the fall in time for the experiment as scheduled.

$$
\star * * * * * * * \hbar \neq * * *
$$

Title of Project: Physics Spectrometer Group, AGS Experiment 594

Person in Charge: S. Ozaki, S.J. Lindenbaum

Scientific and Professional Staff: K.J. Foley, J. H. Goldman, W.A. Love,

E.D. Platner (Physics Department), A. Etkin, T.W. Morris,, A.C. Saulys, C.D. Wheeler, E.H. Willen (Accelerator Department)

\section{Scope of Work}

While this group of physicists, which represents both physics and Accelerator Departments, is responsible for the technical operation, maintenance and improvement of the MPS facility, they also carry out a particle physics research program with it. During the last year this group, in collaboration with a CCNY group, carried out the first half of the AGS Experiment 594, entitled "A Survey of Strange and Non-strange Boson Production at MPS." The first phase of it was. $\sim 200$ hour run with $6 \mathrm{GeV} / \mathrm{c} \mathrm{K}^{-}$beam on hydrogen target with trigger which enabled us to select $\mathrm{K}^{\circ} \pi^{+} \pi^{-}$decay of $\mathrm{K}^{*_{0}}$. This run yielded more $\mathrm{K}^{\circ} \pi^{+} \pi^{-}$events than the world total data to date in this reaction. 


\section{BROOKHAVEN NATIONAL LABORATORY \\ Upton, New York}

We plan to improve the data statistics by an order of magnitude and hope to settle conclusively the muddled status of $K^{*}$ spectroscopy.

Goals and/or Major Results to Date

A 200 hour data run on Experiment 594 yielded high statistics sample of $\mathrm{K}^{-} \mathrm{p} \rightarrow \mathrm{K}^{\mathrm{O}} \pi^{+} \pi^{-} \mathrm{n}$ samples ( 10,000 events). In the $\mathrm{K}^{\circ} \pi^{+} \pi^{-}$effective mass spẹctrum thus obtained, we observe, in addition to the we 11 known $K^{\star}(1420)$ peak, a $6.6 \cdot s t a n d a r d$ deviation peak at the mass of $1693 \mathrm{MeV}$ suggesting a new $K^{*}(1700)$ resonance. One of the significant characteristics of this peak is that it is rather narrow $\left(\Gamma=26_{-17}^{+24} \mathrm{MeV}\right)$. Further analysis of the data from this run is in progress.

$$
* * * * * * * * * * * * * * *
$$

Title of Project: Physics Spectrometer Group, High Pressure Cerenkov Counter Hodoscope

Person in Charge: S. Ozaki, S.J. Lindenbaum

Scientific and Professional Staff: K.J. Foley, J.H. Goldman, J.T. Koehler, W.A. Love, E.D. Platner (Physics Department), A. Etkin, T.W. Morris, A.C. Saulys, C.D. Wheeler, E.H. Willen (Accelerator Department)

\section{Scnpe nf Wark}

The new high pressure Cerenkov counter will operate at up to 60 psig. Using Freon 12 gas, this will give a threshold of $\sim 1.4 \mathrm{GeV}$ for pions, a region well matched for reactions with multi-bndy final states in the MESB. The geometry of the counter has been optimized for use with the BNL MPS with efficient light collection for particle trajectories which exit from the downstream edge of the MPS magnet. In order to accommodate the necessary wide range of angles $\left(\sim \pm 20^{\circ}\right)$ a novel light collection scheme is employed: in the horizontal projection planar optics are used, giving position sensitivity, while in the vertical projection the smallness of the bending in the magnetic field permits the use of eliptical focussing, resulting in a drastic reduction in the number of phototubes required. The use of this counter will permit the study of high multiplirity final states and, in combination with kinematic restraints will permit complete reconstruction of such events in an energy region not previously studied. The first use of the counter will be in Experiment 682 where it will be used together with the existing atmospheric Cerenkov counter to separate pions, kanns, and antiprotons from 5 to $10 \mathrm{GeV} / \mathrm{c}$. 
BROOKHAVEN NATIONAL LABORATORY

Upton, New York

Goals and/or Major Results to Date

Design of the optics and pressure vessel is complete. The vessel is at an advanced stage of construction and will be finished later this year. We are currently studying modern production methods in plastic forming in order to minimize the cost of the optical components. We expect delivery of the optical components later this year.

$$
* * * * * * * * * * * * * *
$$

Title of Project: High Energy Physics Theory Group

Person in Charge: T.L. Trueman, Group Leader

Scientific and Professional Staff: R.F. Peierls, I.J. Muzinich, L.L. Wang, R.M.: Sternheimer, F.E. Paige, M. Creutz, E.A. Paschos, D.P. Sidhu

\section{Scope of Work}

The general aim of the group's work is to understand the properties of elementary particles, in terms of both general, fundamental theory and the analysis of specific experimental results. The areas of study at the present time include:

1. The production of new particles (charm, J/ $\Psi$ ), dileptons, $\mathrm{W}$ mesons and particles with high transverse momenta;

2. Weak neutral currents in neutrino reactions;

3. Spectra and decays of new particles;

4. Production of particles by strong interactions at high. energy (Regge : theory);

5. Polarization effects in elementary particle reactions;

6. Beam stability in the proposed ISA;

7. Fundamental structure of elementary particles (field theory, quark confinement, solitons, lattice theories);

8. Implications of new theories of elementary particles for weak interactions;

9. Atomic physics (fine and hyperfine structure, shielding and antishielding). 


\section{BROOKHAVEN NATIONAL LABORATORY}

Upton, New York

There are members of the group involved in most of the areas of high energy theoretical physics. The specific problems change as new experimental results and theoretical ideas become available.

Goals and/or Major Results to Date

Over the years the group has made many important contributions to the gradual understanding of high energy physics, especially in the areas of strong interaction scattering and production processes (Regge theory), spin problems, neutrino reactions, and field theory. The group has a otrong program of summer visitors from all over the world who have made many important contributions to all areas of high energy theory during their stay here. Our general goals were given at the beginning and the specific goals are implicit in the current studies listed ahnve. One other epccific goal that should be mentioned is planning for the utilization of the proposed ISA.

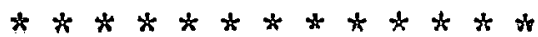

Title of Project: Alternating Gradient Synchrotron (AGS) Operations and $R \& D$

Person in Charge: M.Q. Barton, J.J. Grisoli, R.R. Rau, L.W. Smith

Scientific and Professional Staff: R. Adams, R. Blumberg, J. Claus, J. Cottingham, D. Davis, W. Gefers, W. Glenn, H. Hsieh, Y.Y. Lee, A.W. Maschke, E. Raka, J. Sheehan, H. Weisberg, G. Bagley, A. Bertsche, M.W. Brown, R. Browne, H. Bumstead, K. Christianson, G. Cornish, T. Coyle, J. Curtiss, J. Dabrowski, A. Feltman, N. Fewe11, J. Gabusi, J. Keane, O. Kochman, R. Lankshear, R. Lockey, F. Martin, A. McGeary, N. Parrine1lo, L. Repeta, E. Rogers, J. Ryan, R. Sanders, J. Schuchman, J.D. Smith, R. Warkentien, T. Wild, R. Witkover.

\section{Scope ot Work}

The Alternating Gradient Synchrotron (AGS) is a $30 \mathrm{GeV}$ proton accelerator which provides high energy particles for physics experiments. The AGS Divisinn operates the $200 \mathrm{McV}$ injector, the synchrotron itself and the external beam systems up to and including the primary beam target stations.

The Division is responsible for accelerator studies directed toward proper operation of the AGS for utilization with the growing experimental program, and for potential long-range use of the AGS as an 
injector synchrotron. The design, development, fabrication and installation of new equipment and improvements to the AGS to match the experimential program are included in this activity as well as the routine maintenance and minor repairs required.

The 200-MeV 1inac, in addition to serving as an injector for the AGS, continues to serve satellite programs using the Brookhaven Linac Isotope Producer (BLIP) and the Chemistry Linac Irradiation Facility. (CLIF). Neutron rich isotopes are being studied with use of both primary proton and secondary neutron bombardment schemes. Radio-isotopes are produced on a regular weekly basis for use in clinical studies in various research hospitals. Average beam intensities of 150 microamps were delivered to'BLIP.

Goals and/or Major Results to Date

The AGS ran for a scheduled High Energy Physics program of 30 weeks in FY 1976, providing both a fast extracted beam for neutrino experiments and slowly extracted beams for counter-electronic experiments. The peak AGS intensity during the period exceeded $10^{13}$ protons per pulse and the average intensity was higher than in any previous period.

Accelerator improvements included changes in many of the subsystems of the $200-\mathrm{MeV}$ linac to increase performance and reliability. The use of an electrostatic septum in the slowly extracted beam(s) system increased the extraction efficiency to approximately $96 \%$ reducing by a factor of two to three the extraction losses and resultant radioactive build-up.

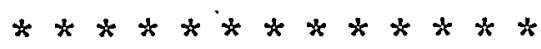

Title of Project: Experimental Planning and Support (EP\&S) operations and $R \& D$

Person in Charge: M.Q. Barton, H.J. Foelsche, J.J. Grisoli, R.R. Rau

Scientific and Professional Staff: J. Allinger; H.N: Brown, A. Carroll, G. Dariby, B, DeVito, D. Lazarus, D. Lowenstein, W.G. Walker, P. Yamin, F. Atkinson, T. Blair, C. Bohnenblusch, L. Chimienti; A. Dick, F. Heimburger, R: Hulliger, J. Jackson, V. Kovarik, V. Lettieri, P. Montemurro, A. Pendzick, C. Rago, W. Sims, A. Soukas, F. Thornhi11, J.C. Walker 


\section{BROOKHAVEN NATIONAL LABORATORY \\ Upton, New York}

Scope of Work

Al1 Alternating Gradient Synchrotron (AGS) experimental beams are constructed, operated and malntained by the Brookhaven National Laboratory (BNL) Experimental Planning and Support (EP\&S) Division. BNL physicists, engineers, and techniclans work with the experimenters in planning, erecting, modifying and servicing experiments.

The EP\&S Division carries out the setup and rearrangements of shielding and large experimental devices, maintenance and operation of beam separators, measurements of magnet characteristics, the setup and malntenance of electric power and cooling water systems, all as required to support the research activities of residont and guest sclentist users from many of the nation's unfversities. Operational crews are maintained on a 24-hour, 7-days-a-wék basis to operate, service and provide safety survelllance of the experimental areas and equipment.

An electronic equipment pool provides fast electronics and other experimental equipment for experimenters at the AGS.

Since the requirements of scientists doing research at the AGS are constantly developing as a result of continuing experience and exploitation of new techniques, a program of experimental facilities and equipment development is required. This R\&D program covers active design and development of beam transport systems, magnets, power supplies, beam separators, and new developments in the area of high electrostatic fields, etc. High-field superconducting and cryogenic magnet design and develnnment ars carricd out with appl1cation to experimental apparatus and beam transport equipment. This work is part of a coordinated BNL program in "cold" technology.

Goals and/or Major Results to Date

In FY 1977, it is planned to operate the experimental program from 30 weeks which should provide a total of $\sim 3000$ particle physics beam hours. Twenty weeks will be devoted to electronic detector experiments using the slow extracted beam and 10 weeks for neutrino physics using the fast extracted beam, both for the 7-foot bubble chamber and for electronic experiments.

The redesign, modification and improvement of existing beam facilities and support for AGS experimenters from in-house and university groups will continue. 


\section{BROOKHAVEN NATIONAL LABORATORY \\ Upton, New York}

Title of Project: Particle Detector (PD) Operations and R\&D

Person in Charge: M.Q. Barton, J.J. Grisoli, R.I. Louttit, R.R. Rau

Scientific and Professional Staff: B. Culwick, A. Etkin, K. Foley, C. Goodzeit, S.J. Lindenbaum, W.A. Love, T. Morris, S. Ozaki, E. Platner, A. Prode11, D. Rahm, A. Saulys, J. Sondericker, W.A. Tuttle, C.D. Wheeler, E. Willen; S. Eiseman, J. Gould, R. Imossi, C.L. Jacobs, R.E. Meier, W.J. Schnelder, G.A: Smith, W. Wilhelm, C. Zein

\section{Scope of Work}

The Particle Detector (PD) Division constructs, operates, and maintains several major facilities for use in high energy particle interaction studies by any approved experimenter. These include the following:

The 7-foot Bubble Chamber provides extremely good spatial resolution and magnetic momentum analysis in a large volume of target material. The chamber is located in a high-intensity beam of neutrinos or antineutrinos, and can be filled with liquid hydrogen, deuterium, or neon/hydrogen mixtures. Its visible volume is 9400 liters, and its magnetic field of 30,000 gauss is provided by a superconducting magnet.

A system of magnetic horns focusses pions in the direction of the 7-foot chamber and other neutrino experiments, and thus determines the intensity and energy-spread characteristics of the neutrino or antineutrino beam. A pair of superconducting beam magnets bends the ejected proton beam from the AGS through an angle of $8^{0}$ toward $\therefore$ the magnetic horns.

The Multiparticle Spectrometer (MPS) is a large volume C-magnet filled with electronic detectors, with other electronic detectors surrounding it. It provides very high rate (and, therefore, high statistics) capability for fairly complicated particle interactions, but somewhat less spatial resolution than the Bubble Chamber.

The On-Line Data Facility (OLDF) provides high on-line computer power to all experimental groups needing it. It consists of two DEC PDP-10 computers with about 400,000 words of memory and appropriate peripheral equipment. These are used by experimenters to monitor the flow of data from their apparatus for relevance to experimental goals, and to make some decisions about acceptance or rejection of events. 


\section{BROOKHAVEN NATIONAL LABORATORY}

Upton, New York

The Target Group builds and operates passive liquid hydrogen and deuterium targets to meet the special requirements of the experiments needing them.

Goals and/or Major Results to Date

The 7-foot Bubble Chamber has been expanded 1.6 million times and taken a total of 750,000 stereo sets of pictures. Most of these have been analyzed yielding many interesting results, and one event in particular seems to be the first.clear example of a "charmed particle" decay.

The initial construction of the MPS was completed in 1975, and the first experimental results were published in the spring of 1976. There is a very full schedule of experiments to be performed in the MPS, and a continuing flow of proposals to use it.

The OLDF has provided service to most of the experiments done at the AGS for the past several years, and has been of crucial importance to many of them.

$$
* * * * * * * * * * * * * * *
$$

Title of Project: Intersecting Storage Accelerator (ISABELLE)

Person in Charge: M.Q. Barton, H. Hahn, J.R. Sanford, J. Spiro

Scientific and Professional Staff: J. Bamberger, D.P. Brown, V. Buchanan, R. Chasman, E.D. Courant, P. Dah1, E.W. Dexter, D. Edwards, R. Franke1, S. Giordano, H. Halama, J. Herrera, J. Humphrey, D. Kassner, J. Kaugerts, C. Lasky, A. McInturff, M. Month, G. Parzen, W.B. Sampson, A. Schlafke, C. Theisen, A. Van Steenbergen, J. Aggus, W. Buxton, R. Damm, D. Gilzinger, E. Jablonski, K. Je1lett, R. Keh1, T. Mullany, R. Nawrocky, K. Robins, M.G. Thomas, R.D. Thomas, A. Werner.

\section{Scope of Work}

The Intersecting Storage Accelerator (ISABELLE) Project consists of the construction of two 200-CeV accelerator rings in a common tunnel adjacent to the Alternating Gradient Synchrotron (AGS), so arranged that protons from the AGS may be injected into each ring, in counter-rotating directions. The protons of about $30 \mathrm{GeV}$ from the AGS will be accumulated to obtain the design current of 10 amps prior to acceleration to final energy. The proton beams will collide at eight interaction regions where particle detectors will 


\section{BROOKHAVEN NATIONAL LABORATORY \\ Upton, New York}

be arranged to study the collision processes. ISABELLE will permit the exploration of proton-proton collisions at center-ofmass energies continuously variable from 60 to $400 \mathrm{GeV}$ and with luminosities of $10^{32}$ to $10^{33} \mathrm{~cm}^{-2} \mathrm{sec}^{-1}$ over the entire range. An important new feature of ISABELIE is the use of superconducting magnets operating at $40 \mathrm{kG}$ in order to improve the operating characteristics and reduce the circumference, electric power consumption, and cost of the machine.

It is expected that the high energies of ISABELLE will provide the experimental information in support of the theoretical attempts to unify the weak and electromagnetic interactions. The performance characteristics of the machine will be adequate for a broad experimental program covering the production of charged and neutral intermediate vector bosons, the hadron production at high transverse momentum, searching for new massive particles, and the energy dependence of the strong interactions.

Goals and/or Major Results to Date

A proposal for construction of ISABELLE at Brookhaven National Laboratory has been submitted to ERDA requesting funding in FY 78 . The conceptual design of the Intersecting Storage Accelerator is completed, and construction planning and design work is in progress in order to sharpen the cost estimate. An intensive research and development program has been directed towards a demonstration of the feasibility of superconducting magnets as required for ISABELLE. Recently, full-size prototype dipoles and quadrupoles together with their cryogenic systems were successfully tested. The construction of a half-cell, the smallest accelerator sub-unit, is in progress.

$$
* \star * * * * * * * * * * * *
$$

Title of Project: Mini-Chamber (New Group)

Person in Charge: R.B. Palmer

Scientific and Professional Staff: R.B. Palmer, M. Montag

Scope of Work

The construction of a small (10 cm diameter, $3 \mathrm{~cm}$ deep) hydrogen bubble chamber with a cycle rate of 3000 expansions per second and a resolution of track positions to the order of 1 micron in space. Currently a prototype expansion system has been built and is under test. 


\section{BROOKHAVEN NATIONAL LABORATORY \\ Upton, New York}

\section{Goals and/or Major Results to Date}

It is hoped to trigger the chamber on charm particle production and observe and measure charm particle lifetimes.

$$
* * * * * * * * * * * * * *
$$

Title of Project: Synchrotron Radiation Research Facility

Person in Charge: M.Q. Barton, M. Blume, G.K. Green

Scientific and Professional Staff: R. Chasman, J. Codel

$\underline{\text { Scope of Work }}$

This project will provide a dedicated complex for research with synchrotron radiation. Research would be carried out in the important fundamental areas of materials, molecular and life sciences as well as investigations relevant to energy and environment. The synchrotron light emitted from an electron storage ring in terms of continuous spectrum, intensity and stability is unique and not available from other sources. At the present time in order to best service the community of users, two storage rings will be constructed. One ring ( $2 \mathrm{GeV})$ would provide the optical characteristics desired by $\mathrm{x}-\mathrm{r}$ ay users and other $(700 \mathrm{MeV})$ for researchers using vacuum ultra violet (VUV) radiation. It is intended to use a $50 \mathrm{MeV}$ linac as an injector into a $700 \mathrm{MeV}$ booster synchrotron. This combination of linac and booster synchrotron then serves as injector to the $2 \mathrm{GeV}$ and $700 \mathrm{MeV}$ storage rings. The complex will also have office and laboratory space in a wing adjacent to the facility structure.

\section{Goals and/or Major Results to Date}

The conceptual design for this facility evolved from an interdepartmental study which started in 1971. The study drew very heavily for ideas and advice from the outside community of potential users. In 1976 this effort culminated in a conceptual design report "Synchrotron Radiation Research Facility." This document is in support of a witcten request to ERDA for construction funds during FY 78 .

$$
* * * * * * * * * * * * * *
$$




\section{BROOKHAVEN NAT IONAT. LABORATORY \\ Upton, New York}

Title of Project: Heavy Ion Fusion (Laser Induced Implosion Fusion)

Person in Charge: M.W. Barton, A.W. Maschke

Scientific and.Professional staff: None

Scope of Work

A small group of investigators have undertaken the task to develop plans for producing high powered pulsed beams of heavy ions from a heavy ion storage ring facility using state-of-the-art technology. The pulsed beam would be used for ignition of inertially confined thermonuclear pellets. Initially, simulation studies will be undertaken of the various accelerators and storage ring devices. Each stage of beam bunching and acceleration will be modeled to establish the parameters for the accelerator - storage ring complex.

Goals and/or Major Results to Date

The efforts over the next few years will be directed toward establishing the conceptual design of the accelerator storage rings complex and coordinating accelerator design concepts with the pellet design groups at other 1 aboratories.

$$
\star * * * * * * * * * * * * *
$$

Title of Project: Electric Power Transmission

Person in Charge: M.Q. Barton, E.B. Forsyth, J. Gurinsky

Scientific and Professional Staff: J. Bussiere, M. Garber, J. Jensen, K. Minati, G. Morgan, J. Rigby, A. Blummert, R. Gibbs, W. Harrison, A. MaNerney, A. Muller.

Scope of Work

The objective of this program is to develop an underground superconducting power transmission cable which is both economical and technically attractive to the utility industry. The cable must be capable of carrying very large blocks of power so that it could replace overhead transmission lines in both rural and urban areas. A superconducting cable section will be built, complete with refrigeration system to test and establish the performance of such a system under actual power transporting conditions. 
BROOKHAVEN NATIONAL LABORATORY

Upton; New York

Goals and/or Major Results to Date

A refrigerator has been set up at the test facility and continuous tèsting under a variety of load conditions is underway. Investigations of superconducting materials and insulation have been performed and this effort will continue to optimize the performance of both. The results of the materials and systems testing should lead to the design and construction of acceptable prototype superconducting transmission cable.

$$
* * * * * * * * * * * * *
$$

Title of Project: Negative. Ion Sources for Fusion Projects

Person in Charge: M.Q. Barton, K. Prelec, Th. Sluyters

Scientific and Professional Staff: M. Grossman, C. Lam, R.

McKenzie-Wilson, R. Larsen

\section{Scope of Work}

This program is intended to develop a $150 \mathrm{keV}$, multiampere neutral injector which is suitable for initial heating experiments in toroidal plasma devices. To achieve this objective, multiampere negative ion sources and accelerating tubes must be developed. Research will include the continued investigation of direct extraction of negative ions from a plasma, various types of focussing devices and neutralizer jets.

\section{Goals and/or Major Results to Date}

During the past few years a high current, $60 \mathrm{~mA}$, hollow discharge, duoplasmatron source has been developed. Most recently a six-slit, long cathode magnetron was designed and tested. $\mathrm{H}^{-}$yields of about 1 ampere in $10 \mathrm{~ms}$ pulses were achieved. A $150 \mathrm{kV}, 1 \mathrm{~A}, 10 \mathrm{~ms}$, negative ion accelerator with a $70,0001 / \mathrm{s}$ cryopump was constructed and made operational.

$$
\star * * * * * * * * * * * * *
$$

Title of Project: Neutron Generator

Person in Charge: M.Q. Barton, J. Hendrie, P. Grand

Scientific and Professional Staff: K. Batchelor 


\section{BROOKHAVEN NATIONAL LABORATORY \\ Upton, New York}

Scope of Work

It is proposed to build a. $30 \mathrm{MeV}$ deuteron linear accelerator with a capability of $100 \mathrm{~mA}$ of current. This beam would be directed on to a flowing lithium target to produce a source intensity of about. $10^{16}$ neutrons per second. This facility would be vital to assess the effects of intense neutron radiation on materials intended for use in the construction of fusion reactors.

Goals and/or Major Results to Date

A proposal titled "Accelerator-Based Neutron Generator" has been written. This document is in support of a written request to ERDA for construction funds during FY 78 .

$$
* * * * * * * * * * * * * *
$$


FERMI NATIONAL ACCELERATOR LABORATORY

Batavia, Illinois

Contract No. EY-76-C-02-3000

Title of Project: Theory

Person in Charge: c. Quigg

Scientific and Professional Staff: W. Bardeen, J. Carazzone, J. Lieberman, R. Pearson, E. Rabinovici, M. Roth, R. Savit, R. Schrock, H. Thacker, and C. Quigg

Scope of Work

Participants in this program pursue basic theoretical physics research in various areas of high energy physics. There is an active visiting theorist program; theoriste from various institutions in the United States and abroad are invited to Fermilab to share their. research results and expertise with the theoretical and experimental scientific staff of the Laboratory, and often to participate in the on-going theory research programs.

In addition to pursuing theoretical research, members of the Fermilab Theoretical Physics staff and visitors frequently consult with their experimentalist colleagues on the Fermilab staff and with the visiting university users. Formal and informal discussions in the form of corridor discussions, seminars, workshops, and mini-conferences are held regularly. The theorists often aid in relating experimental results to theoretical models. Some members of the department participate actively with the Directorate and the Program Advisory Committee in the evaluation of experimental proposals.

Goals and/or Major Results to Date

During FY 76, members of the Fermilab Theoretical Physics Staff and visitors were engaged in, and produced important results in, the following research topics: unified gauge theories of particle interactions; theoretical investigations of dimuon events, and neutrino interactions; phenomenology of charmed particles; study of quark confinement, etc.

The research topics listed above are of continuing interest as they involve crucial questions at the forefront of our understanding of the structure of matter.

$$
* * * * * * * * * * * * *
$$


FERMI NATIONAL ACCELERATOR LABORATORY

Batavia, Illinois

Title of Project: Physics. Research - Electronic Detector Experiments

Person in Charge: F.A. Nezrick, P. Koehler

Scientific and Professional Staff: C. Ankenbrandt, J. Appe1, E. Bleser, H. Edwards, G. Fisk, R. Johnson, S. Pruss, R. Stiening, F. Turkot, R. Yamada; D. Johnson; H. Barton, M. Johnson, R. Loveless; R. Orr; A. Brenner, D. Carey; A. Greene, T. Yamanouchi; P. Limon, A. Tollestrup; T. Nash; C. Brown, S. Ecklund, J. Elias, H. Haggerty, E. Malamud, L. Read, A. Wehmann; R. Lundy, S. Mori, R. Stefanski, D. Theriot, H. White; J. MacLachlan; R. Carrigan; V. Ashford, D. Bintinger, B. Brown, D. Edwards, I. Gains, W. Innes, A. Jonckheere, P. Koehler, T. Kondo, A. Lennox, G. Mikenberg, D. Nease, R: Rubinstein, G. Shen, J. Tompkins, J. Walker; P. Gollon; B . Cox, D. Eartly, P. Garbincius, J. Lach, P. Mazur, T. Murphy, A. Roberts, R. Sorber; W. Baker, D. Jovanovic, J. Peoples, T. Toohig; M. Atac, M. Binkley, R. Fast, P. Mantsch, R. Shafer.

Scope of Work

This contract covers the support of the experimental research programs of those Fermilab staff physicists who utilize electronic techniques of particle detection. At any given time these physicists are involved in a total of $20-25$ separate experiments. Almost all of them are performed in collaboration with physicists from institutions outside Fermilab, both U.S. and foreign.

\section{Goals and/or Major Results to Date}

The major accomplishments include:

A. Differential cross sections for the elastic scattering of $\pi^{ \pm}, K^{ \pm}, p$ and $\bar{p}$ from hydrogen and deuterium targets have been determined throughout the range $0.001<|\mathrm{t}| \leq 2.0(\mathrm{GeV} / \mathrm{c})^{2}$, for incident beam momenta between 50 and $200 \mathrm{GeV} / \mathrm{c}$.

B. Several multiparticle experiments have searched for evidence of charmed particles and studied the production and decay properties of the $\Psi / J$ particles.

C. The total cross sections for $\nu$ and $\bar{v}$ interactions have been determined between 80 and $250 \mathrm{GeV} / c$, with greatly improved absolute normalization. 
FERMI NATIONAL ACCELERATOR LABORATORY

Batavia, Illinois

D. A more accurate value for the electromagnetic radius of the negative pion has been deduced from a measurement of the form factor over a wider range of momentum transfer.

$* * * * * * * * * * * * * * *$

Title of Project: Physics Research - Bubble Chamber Experiments

Person in Charge: F. A. Nezrick, P. Koehler

Scientific and Professional Staff: R. Huson; R. Walker; E. Malamud; R. Lundy, W. Smart, R. Stefanski, L. Voyvodic; J. Lach, T. Murphy; P. Berge, D. Bogert, F. DiBianca, R. Hanft, R. Harris, S. Kahn, J. Lys, R. Nezrick, R. Raja, W. Scott, J. Wolfson.

Scope of Work

This contract covers the support of the experimental research program of those Fermilab staff physicists who utilize the 15-ft. bubble chamber and 30-in. bubble chamber in their experiments. Most of these experiments involve collaborations with university groups. The neutrino experiment involves a collaboration with USSR groups. All film-oriented experiments are scanned and measured utilizing a common facility, the film analysis facility (FAF) of the Physics Department. Considerable advances have been made in FAF in the development of a semi-automatic measuring system and in promulgating a high quality large bubble chamber geometrical reconstruction program.

Goals and/or Major Results to Date

The major accomplishments include:

A. Neutrino Experiments in the 15-Pt. Bubble Chamber

1) Neutrino interactions in hydrogen (E45).

2) Antineutrino interactions in hydrogen-neon (E180).

B. Hadronir. Interacliuns in the 15-lit. and J0-Iuch Bubble Chambers

1) $\pi-p$ interactions at $250 \mathrm{GeV} / \mathrm{c}$ (E234), 15-ft. bubble chamber

2) $p-p$ interactions at $300 \mathrm{GeV} / \mathrm{c}$ (E37)

3) $\mathrm{p}-\mathrm{d}$ and $\pi-\mathrm{d}$ interactions at $100 \mathrm{GeV} / \mathrm{c}$ and $400 \mathrm{GeV} / \mathrm{c}$

4) $\bar{p}=\mu$ Inleractions at $100 \mathrm{GeV} / c$. 


\section{FERMI NATIONAL ACCELERATOR LABORATORY \\ Batavia, Illinois}

C. Development of the Physics Department Film Measuring

Facility

$$
* * * * * * * * * * * * *
$$

Title of Project: Meson Department

Person in Charge: Charles N. Brown

Scientific and Professional Staff: Charles N. Brown

$\underline{\text { Scope of Work }}$

The Meson Area encompasses six secondary beam lines emanating from a single high intensity particle production target, a large enclosed area - the Detector Building - housing approximately 16 high energy experimental stations, and the supporting power, water and control facilities needed to effectively carry out high energy research. The scope of this budget activity includes the instal: lation, operation, maintenance, and improvement of the Meson Laboratory secondary beam lines and experimental areas with the goal of optimizing conditions for high energy physics research.

\section{Goals and/or Major Results to Date}

At the end of the first three quarters, nineteen different experiments had taken data during FY 76. Of these, seven completed their approved experimental program. A list of the nineteen experiments follows:

Experiment

324

236

61

216

86

268

357

8

248

397

366

425

226

330
Short Title

Date Completed

Inclusive Scattering

Hadron Tests

Polarized Scattering

Form Factor

Pion Dissociation

Inclusive Photon

Particle Search

Neutral Hyperon

Neutron Elastic Scattering

Particle Search

Particle Search

$\mathrm{K}^{\mathrm{O}}$ Regeneration

$\mathrm{K}^{\mathrm{O}}$ Electron Regeneration Tests

Particle Search
1 October 1975

24 March 1976

11 February 1976

24 March 1976

7 July 1975 
Experiment

395

260

69

118

178
Short Title

Date Completed

25 February 1975

14 August 1975

The increased energies and secondary particle fluxes in the six beam 1 ines have resulted in most experiments being able to study reactions at higher energies than was anticipated in the original proposals. Un the other hand, pulsed operation of all major beam line magnets in the Meson Laboratory has made it possible to utilize this increase in secondary particle energies without significant increascs in electrical power consumption.

$$
* * *+* * * * * * * * * * .
$$

Title of Project: Neutrino Area Operation - Electronic Detector Experiments

Person in Charge: R.R. Wilson

Scientific and Professional Staff: D. Theriot, R. Stefanski, S. Mori

Scope of Work

This activity includes the installation, operation, maintenance, and improvement of the serondary beamo and clectronic exuerlments approved for running in the Neulrino Area. The facility for handling and repair of the radioactive target systems and other components is also included. These beams serve the 15-Ft. and 30-In. Bubble Chambers as we 11 as electronic counter experiments. Three laboratory buildings housing five major electronic detector experiments are also operating.

Goals and/or Major Results to Date

During FY 76 the neutrino and muon beam lines were operated at higher energico and intensities lhan cver before. This was in part due to high energy operation of the accelerator, and in part to improvements in the beam lines and associated systems. The dichromatic neutrino beam was operated at $250 \mathrm{GeV} / \mathrm{c}$, the horn neutrino beam Iine at $500 \mathrm{GeV} / \mathrm{c}$, the muon beam line at $275 \mathrm{GeV} / \mathrm{c}$, and the hadron lines at $400 \mathrm{GeV} / \mathrm{c}$. Goals are continued operation as the experimental program demands.

$$
* * * * * * * * * * * * *
$$


FERMI NATIONAL ACCELERATOR LABORATORY

Batavia, Illinois

Title of Project: Neutrino Area Operations - Bubble Chamber Experiments

Person in Charge: R.R. Wilson

Scientific and Professsonal Staff: D. Theriot, G.T. Mulholland

Scope of Work

This activity includes the operation and maintenance of the 15-Ft. and 30-In. Cryogenic Bubble Chambers and the particular auxiliary detection systems related to the chambers. The responsibility of the national supply of liquid neon and its maintenance is included.

Goals and/or Major Results to Date

During CY 75 the 30-In. Bubble Chamber produced 900 thous and pictures at various momentum with beam particle tagging. The 15-Ft. chamber produced 300 thousand. The 15-Ft. chamber operated at full magnetic field ( $30 \mathrm{kG})$ and $r$ an with hydrogen and $65 \%$ neon-35\% hydrogen fills. The external muon identifier (EMI) was operated during these pictures. Goals are continued routine operation with hydrogen, deuterium, and neon-hydrogen mixtures.

$$
* * * * * * * * * * * * * * *
$$

Title of Project: Proton

Person in Charge: B. Cox

Scientific and Professional Staff: C.T. Murphy, D. Eartly, A. Roberts, J. Lach, P. Mazur, P. Garbincius

Scope of Work

The scope of this activity includes the installation, operation, maintenance, and improvement of the primary proton beams immediately upstream of the Proton Area, the target stations, the secondary beams, and the experiments approved for running in the Proton Area.

The Proton Area was designed to provide facilities for doing certain classes of experiments that cannot be accommodated conveniently in the Meson and Neutrino Areas. The common thread which runs through these classes of experiments is the need for a proton $b$ am which can be varied over a substantial range of intensity and tuned over a variety of spot sizes. In the course of the Proton Area program 
FERMI NATIONAL ACCELERATOR LABORATORY

Batavia, Illinois

to date, measurements have been made with as few as $10^{9}$ protons per pulse and as many as $5 \times 10^{12}$ protons per pulse, and beam spot sizes from $0.016-$ by $0.100-i n$. to $0.030-$ by $0.040-i n$. In these experiments the choice of parameters is critical and compromises are not possible.

The Proton Area is currently organized around three independent target stations. (The associated experimental areas are designated Proton-East, Proton-Center, and Proton West.) The target systems used are quite different from those 1 in the Meson and Neutrino Areas. The components for the systems in Proton-Center and Proton-East. are placed inside a sleel box 15-in. high and 60-in. across with walls that are 1-ft. thick. The depth of the box in the direction of the incident proton beam is 22- $\mathrm{ft}$. in P-Center and 44- $\mathrm{fl}$. In P-East.

Goals and/or Major Results to Date

A total of nine major experiments have taken data and three have been completed. The area has taken beam at 200,300 , and $400 \mathrm{GeV}$ and has utilized the front porch of the accelerator with all three areas operating simultaneously.

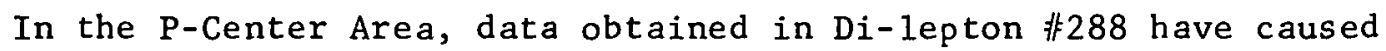
great excitement--the observance of an extremely high mass stage at $6 \mathrm{GeV}$ has been reported. This experiment is unique in its ability to probe the highest mass regions and to study previously unreported mass structures such as the $6 \mathrm{GeV}$ object. Additional structure above and below the $6 \mathrm{GeV}$ reginn has been reported fromi this experiment and continues to he undor otudy in the dl=electron phase of the experiment. This group is currently preparing to switch to the di-muon phase of their program.

In the East Area, the Broad Band Neutron-Photon beam continued to operate successfully and was utilized in Photoproduction .\# 87 for. the study of the $\Psi(3.1)$ and $\Psi^{\prime}$ (3.7) narrow, high mass resonances and in Di-muon 3358 for neutron excitation of the $\Psi$ (3.1). Hints. of a possible structure at $4.7 \mathrm{GeV}$ have been reported from this work.

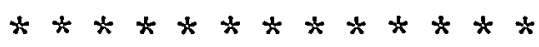

Title of Project: Research Services - Administration

Person in Charge: Q. Kerns

Scientific and Professional Staff: P. Mantsch, R. Shafer, C. Anderson, L. Mapalo 


\title{
FERMI NATIONAL ACCELERATOR LABORATORY
}

\author{
Batavia, Illinois
}

Scope of Work

The Research Services Department provides services to the various groups carrying out experiments. These services include surveying and alignment, maintenance for PREP equipment, maintenance for test instruments such as oscilloscopes and meters, cryogenic magnet and target design and maintenance, beam line control system development and maintenance, detector development, and various electronic services.

Generally, the visiting experimenters are able to secure most services needed to set up and maintain their experiments from this Department.

\section{Goals and/or Major Results to Date}

The support services activities will increase in several areas appropriate to the expansion in the experimental program. Continued effort will be maintained to improve helium recovery efficiency because there will be additional cryogenic magnets in service. Surveying and alignment wịll be facilitated in radioactive areas by the addition of portable surveying instrumentation using vidicon camera and remote T.V. readout, thus reducing radiation exposure to a minimum. The Beam Systems operation will add a second PDP11/45, with. 25 megawords of disk plus the interface parts and DEC Net hardware to permit the auto dialing feature for automatic paging and interconnecting machines.

$$
* * * * * * * * * * * * * *
$$

Title of Project: Alignment

Person in Charge: C. Testin

Scientific and Professional Staff: T. Tópolski

\section{Scope of Work}

The Alignment group is responsible for the surveying and alignment of all magnets (both accelerator and beam line), targets, detectors and other devices necessary to perform the experiments or operate the accelerator. This group also maintains a calibration facility for the optical equipment which is used in their work. 
Goals and/or Major Results to Date

The Alignment group has been instrumental in bringing the acce1erator to its present state of operation by the precise alignment of magnets and beam monitoring devices.

Future goals are to continue providing service to the experimental groups which will enable them to successfully complete their $\cdots$ experiments, and to continue the routine alignments necessary to the operation of the accelerator.

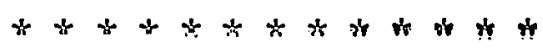

Title of Project: Beam Systems

Person in Charge: M. Johnson

Scientific and Professional Staff: E. Arko, E. Barsotti, H. Barton, J. Bobbitt, M. Haldeman, T. Lahey, R. Loveless, D. Purvis, J. Simanton

\section{Scope of Work}

The Beam Systems group has the controls responsibility from the Switchyard on through all the experimental areas. Development of equipment to provide the accurate control of the beam lines is a major function of this group.

Goals and/or Major Results to Date

A PDP-11 for beam line control is now installed in the 13th floor of the Central Laboratory Building and initial operation begun. XDS530 consoles have been developed and installed in the Meson and Proton areas. A program to reduce failure rates and upgrade system reliability is underway.

$$
* * * * * * * * * * * * *
$$

Title of Project: Cryogenics

Person in Charge: R. Fast

Scientific and Professional Staff: M. Binkley, H. Hart, J. Heim, E. Leung, J. Peifer 


\section{FERMI NATIONAL ACCELERATOR LABORATORY}

Batavia, Illinois

\section{Scope of Work}

The Cryogenics group is charged with responsibility for the deve1opment and construction of cryogenic analysis magnets. Hydrogen targets are also installed and operated by this group. A major effort is expended in operating the helium liquifier. This facility supplies the liquid helium for the cryogenic magnets by recovering the boil-off gas from the magnets and re-liquifying it at a great savings to the Laboratory.

Goals and/or Major Results to Date

Eight medium and large targets were installed and operated during the past fiscal year in addition to several smaller ones. These targets were all maintained by the Cryogenics group. Monitoring systems for all targets and magnets continued to be designed and built.

Three large analysis magnets were put into service in FY 76 . The $24 \times 8 \times 72$ magnet (Delta) was completed, and a design study was made of a cold-iron $24 \times 12 \times 72$ magnet (Echo). Specifications for the coil and cryostats were written and quotations for four such magnets were requested. A feasibility and preliminary design study was begun on a $32 \times 32 \times 60,40 \mathrm{kG}$ analysis magnet.

In response to the expanding use of these large cryogenic magnets, concerted efforts were undertaken to upgrade the equipment and techniques necessary to recover and re-liquify boil-off helium with a very high efficiency. Recovery techniques learned will be critical to the operation of the Energy Doubler/Saver where the helium use will increase a hundred fold.

$$
* * * * * * * * * * * * *
$$

Title of Project: Detector Development

Person in Charge: M. Atac

Scientific and Professional Staff: M. Atac

Scope of Work

Detector Development is a small group whose function is to develop and supply various types of detectors to the experimental groups. A great deal of effort goes into the study of the physics of the chambers and the associated electronics. A large chamber winding facility is run by this group. 
FERMI NATIONAL ACCELERATOR LABORATORY

Batavia, Illinois

Goals and/or Major Results to Date

Emphasis has been placed on drift chamber development including both the physics of the chambers and the readout electronics.

The Detector Development group organized a Drift Chamber Conference and published a report. Both commercial (LeCroy 2770) and Fermilab drift chamber digitizers were evaluated, and numerous improvements suggested as a result of experience in the beam lines. A large drift chamber winding facility was developed in FY 76. It will be possible to construct chambers as large as $10 \mathrm{ft} . x 12 \mathrm{ft}$. using this machine.

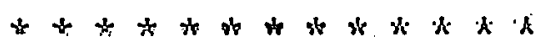

Title of Project: Electronic Development

Person in Charge: $R$. Yarema

Scientific and Professional Staff: K. Seino

Scope of Work

The Electronic Development group is concerned with solving a variety of complex electronic problems. Control-type electronics are especially familiar to this group; monitoring problems associated with hydrogen targets constitutes a major activity.

Goals and/or Major Results to Date

The Electronic Development group has provided interface electronics for the monitoring and control of cryogenic targets and superconducting analysis magnets to aid in integrating these devices into the control system. Improved controls for the helium gas purifier were designed and installed, permitting more He throughput as required to re-liquify boil-off from the superconducting magnets.

$$
* * * * \hbar * * * * * * * * *
$$

Title of l'roject: Experimental systems support

Person in Charge: T. Droege

Scientific and Professional Staff: T. Droege 


\section{FERMI NATIONAL ACCELERATOR LABORATORY}

Batavia, Illinois

Scope of Work

Experimental Systems Support is a very specialized electronic problem solving group. Fast electronics such as that used in data taking by the experiments is developed by these people. Readout systems for detectors is a major project of this group.

Goals and/or Major Results to Date

Several specialized modules, particularly useful to the Fermilab program, were developed by this group and built by commercial firms. A continuing program designed to satisfy the needs of experimenters in the electronics area is planned for the future.

$$
* * * * * * * * * * * * *
$$

Title of Project: PREP \& Instrument Facility

Person in Charge: A. Neubauer

Scientific and Professional Staff: C. Andrle, D. Fearnley, M. Kibilko

Scope of Work

This group has the responsibility for the purchase, disbursement, and repair of all Fermilab-owned (PREP) electronic equipment used by the various experimental groups. Test equipment repairs and calibration as well as printed circuit fabrication is also a function of this group.

Goals and/or Major Results to Date

Fast electronics equipment' was provided for approximately 55 experiments. This equipment typically consists of commercialtype devices which are built to Fermilab specifications. New designs are evaluated, and in many cases recommendations are made to manufacturers for needed improvements. Newly purchased equipment is tested and then distributed to the requesting experimenters. Approximately $70 \%$ of the $\$ 4.9$ million inventory is currently in use by various experiments. Fermilab Departments (15 different setups) are using $16 \%$, and the remaining $14 \%$ represents equipment presently in transition between users, and backup equipment to supply emergency needs of experiments. 
The Instrument Facility is included in this activity and is closely related to PREP. Over 3,500 pieces of commercial test apparatus were repaired and calibrated in FY. 76. This equipment ranges from volt-ohm meters to complex CAMAC digital.systems.

During FY 76, approximately 5,000 single and double-sided P.C. boards were produced in the relocated facility in the Village. These new facilities permit faster turn-around time due to less crowded conditions and availability of raw P.C. stock in the same location.

Personnel will assist in the test and development of new electronic. modules, continuing work on drift chamber readout systems especially. Computerized testing of NIM and CAMAC modules will be pursued as tunding permits.

$$
* * * * * * * * * * * * *
$$

Title of Project: Computing

Person in Charge: A.E. Brenner

Scientific and Professional Staff: Dave Carey

Scope of Wnrk

This activity covers the Laboratory's general computing operations. These operations encompass two major types of computing: fast turn-around computing for the support of experiments in data taking operation; and, batch processing for the analysis of the major quantity of data acquired at Fermilab.

For fast turn-around computing it is essential to provide adequate computing power with sufficiently fast response to enable the quality of the data being taken to be assessed by quickly processing a small sample. Initially (starting in late 1973) the Laboratory provided a CDC 6600 with 131,000 words of memory to serve in this capacity. That computer became saturated at the beginning of 1975 . To alleviate this condition a second CDC 6600 with 65,000 words of memory was acquiled in mid-19\%.

The two machines are operated in a joined configuration with a shared storage file system. In this configuration they are interchangeable for all practical purposes. The configuration is such that the permanent records of data or programs available to one computer are also available to the other. In the event of a failure 


\section{FERMI NATIONAL ACCELERATOR LABORATORY}

Batavia, I1linois

of one of the two systems, the remaining computer continues to function'without interruption. In addition to the obvious difference in memory size, there are some slight differences in the configurations of the two machines. Although this restricts some jobs only to one of them, by and large most jobs run equally well on either computer.

Goals and/or Major Results to Date

During the course of FY 76, some major additions and also a reorganization of the hardware configuration were made to the central computing facility at Fermilab. A second small CDC 6600 which had been acquired at the end of FY 75 was installed and brought into operation. This addition improved the work load capability of the previous installed system by about 30\%. Furthermore, during the third quarter of the year, the two systems were loosely coupled together in a dual system configuration to further increase the capability of the overall system; these changes were crucial in view of the saturated condition of the original CDC 6600 that had existed at the beginning of FY 76. These modifications and the additions were essential to satisfy at a minimal but acceptable level the computing requirements necessary for properly analyzing the large amount of data being acquired in the experimental program.

Other accomplishments include the enhancement of the PDP-10 time sharing system with both memory and disk to accommodate the higher activity level on that facility, and the development of a remote job entry terminal with general capabilities which will reduce the need for additional input and output equipment on the central computing facility in a relatively cost effective manner.

$$
* * * * * * * * * * * * *
$$

Title of Project: Advanced Projects

Person in Charge: L.C. Teng

Scientific and Professional Staff: F. Cole, T. Collins, D. Johnson, S. Ohnuma, A. Ruggiero, S. Snowdon, R. Yamada, H. Ishimoto, M. Price

\section{Scope of Work}

This group directs its effort of research and development towards the theory and design of accelerators and the measurement of magnetic fields of magnets. Recent work with ANL has produced 
a proposal for a colliding beam accelerator of $1000 \mathrm{GeV}$ on 1000 $\mathrm{GeV}$ (POPAE). Design continues on various detailed aspects of the Energy Saver/Doubler. Other work has included the normal problems associated with the present accelerators and design and measurement of new magnets. For example, many field measurements and short sample tests have been made for the doubler magnets.

Goals and/or Major Results to Date

During the next year much work will be devoted to colliding beams. For example, various methods to improve intensity (luminosity) including "cooling" protons and antiprulons with electrons, multistacking of beam, etc.

$$
* * * * \ldots * * * * * * * *
$$

Title of Project: Booster Group

Person in Charge: Rolland Johnson

Scientific and Professional Staff: C. Ankenbrandt, J. Bridges, E. Gray, J. Griffin, G. Nicholls

$\underline{\text { Scope of Work }}$

This group directs its effort of research and development towards the $200 \mathrm{MeV}$ proton transport, the $8 \mathrm{GeV}$ booster synchrotron accelerator, and the $8 \mathrm{GeV}$ proton transport to the main accelerator. Recent work has been in the following areas: increasing the acceptance of the $200 \mathrm{MeV}$ line and the booster; varions schemes for injection into the booster from the linac; magnetic field corrections for the booster; the study of the ferrite used in the rf runners; the suppression of transverse instabilities using active feed-back; and cures for longitudinal instabilities associated with the rf capture of the linac beam in the booster.

Goals and/or Major Results to Date

The immediate goal of the booster group is to inject a sufficient number of protons into the main synchrotron ring to allow greater than $5 \times 10^{13}$ protons per pulse to he extracted. Work is progrcasing on the necessary changes to the booster for negative ion injection. Studies are also being done to understand the feasibility of raising the booster energy to $10 \mathrm{GeV}$. Another goal is to provide reverse injection for the main ring. This will be used in the future for colliding beams in the main ring tunnel, for example, between the main ring and doubler.

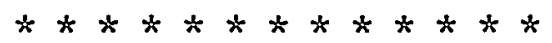


FERMI NATIONAL ACCELERATOR LABORATORY

Batavia, Illinois

Title of Project: Cancer Therapy Department

Person in Charge: L. Cohen, M.D., and M. Awschalom

Scientific and Professional Staff: S. Baker, L. Coulson, P:

Gollon, C. Moore, D. Riley, A. Van Ginneken, S. Velen,

I. Rosenberg

Scope of Work

Cancer Therapy - An out-patient facility has been created in which clinical research is conducted on neutron therapy for the control of certain types of cancers. At this time patient eligibility to the rando ized trials is described in four national protocols prescribing only radiotherapy. Several additional protocols are being developed in which chemo- and radiotherapy are combined. For these studies the neutrons are created by $66 \mathrm{MeV}$ bombarding a $2.21 \mathrm{~cm}$ thick target. This use for the protons is compatible with Fermilab's mission of supporting high energy physics research.

Goals and/or Major Results to Date

Radiation Physics - Radiation safety services are rendered to the whole Laboratory. In addition, instrumentation is developed, Monte Carlo Calculations are made for shielding, detector, and dosimetry calculations, as well as measurements are made to test the validity of the calculations.

$$
* * * * * * * * * * * * *
$$

Title of Project: Controls

Person In Charge: M. Gormley

Scientific and Professional Staff: W. Deluca, R. Ducar, N. Lau, M. Shea, M. Storm, A. Turmillo

Scope of Work

This group directs its effort of research and development towards the controls of the accelerator. Much of the work is maintenance and modification for improved reliability. Recent development has been the consolidation of the computer system to one type of medium calculating computer, plus one type of small data gathering computer. Repeaters have been installed around the 4-mile circumference of the main ring to improve reliability of signal transmission. 
FERMI NATIONAL ACCELERATOR LABORATORY

Batavia, Illinois

Development work has continued on the general Laboratory fire alarm system, FIRUS.

Goals and/or Major Results to Date

Research and development during the next year will be done on the Energy Saver/Doubler system. Work will also be done on instrumentation for the accelerator.

$$
\dot{*} * * * * t * * * * * * *
$$

Title of Project: Electrical Support

Person in Charge: G. Tool

Scientific and Professional Staff: J. Dinke1, A. Donaldson, E. H1gg1ns, Jachim, B. Sandberg, D. Wolff

Scope of Work

This group directs its effort of maintenance and development towards the electrical problems of the accelerator. Recent major efforts include modification and development of a new module for the main ring power supply, there are $360 \mathrm{SCR}$ modules used in the main ring power supply; development of a new SCR switch for the series capacitor bank used for the main $r i n g$; installation of a 47th harmonic filter in the main accelerator power system to avoid resonance production on the Chicago power grid; work on the main accelerator power distribution system to increase its capacity; and design and construction of power supplies for various magnets, from very fast supplies (nanosecond response) to DC supplies .

Goals and/or Major Results to Date

Future design and development will include work on sophisticated instrumentation for accelerator beam detection, further work on special power supplies including power supplies for superconducting magnets, development of rf cavities for the doubler acceleration, and study of high current electron beams for "cooling" of protons.

$$
* * * * * * * * * * * * * *
$$


Title of Project: External Beam Systems

Person in Charge: H. Edwards

Scientific and Professional Staff: J. Appe1, B. Brown, J. McCarthy, J. Walton

Scope of Work

This group directs its effort of research and development towards extraction of protons from the accelerator, splitting of the extracted beam to the various experimental areas, and focussing the beam onto the primary experimental targets. Recent research and development has included work on an extraction system from the main ring for injection into the doubler; tests of new electrostatic septa wire materials and of shadowing targets in front of the septa to protect the wires; operation of superconducting magnets with beam being targeted in front of the magnet to study radiation heat loss in the magnet and corresponding quenching of the magnet; tests of a new superconducting magnet for external beam lines; development of a helium liquefier for general use in the Laboratory; and modifications to give different types of beam spills, from 0.0005 second high intensity to 2-second flattops.

Goals and/or Major Results to Date

The immediate goal is to improve extraction so that more beam intensity can be delivered to the experimental areas.

Future goals include conversion of much of the switchyard to superconducting magnets in preparation for the Energy Saver/ Doubler.

$$
* * * * * * * * * * \star * * *
$$

Title of Project: Internal Target

Person in Charge: T. Nash

Scientific and Professional Staff: L. Olesiuk

Scope uf Work

The research and development efforts of this group are directed toward the experimental high energy physics program performed by using the circulating accelerator beam. This group has recently 
brought into operation the high resolution recoil spectrometer which consists of superconducting magnets. A new gas target jet has been developed and tested and is now used in normal operation. The liquid hydrogen target jets continue to be used for various experiments.

Goals and/or Major Results to Date

Studies are being made to understand the class of future experiments that can best be done in the Internal Target. This includes experiments with the Energy Saver/Doubler and with colliding beams.

$$
* * * * * * * * * * * * * *
$$

Title of Project: Linac

Person in Charge: C.W. Owen

Scientific and Professional Staff: C. Surtis, C. Echmidl, F. Cilyo Scope of Work

This group directs its effort of research and development towards the proton source, the 750 kilovolt Cockcroft-Walton and the 200 $\mathrm{MeV}$ linac. Recent work on the 750 kilovolt column and the rf systems has resulted in a peak proton curreul vui of the linac of 270 miliiamps in 4 microsecond pulses. A beam of $66 \mathrm{MeV}$ protons has just been extracted to be used for producing neutrons for cancer therapy. There will soon be additional extracted beam of $200 \mathrm{MeV}$ protons for use in radiography.

\section{Goals and/or Major Results to Date}

During the next year the goal will be to obtain a negative hydrogen ion beam of $200 \mathrm{MeV}$ from the linac of 30 milliamps in a 50 microsecond pulse. This beam will be used for injection into the booster.

$$
* * * * * * * * * * * * * *
$$

Title of Project: Main Ring

Person in Charge: F. Turkot

Scientific and Professional Staff: E. Bleser, H. Miller, H. Pfeffer, S. Pruss 


\section{FERMI NATIONAL ACCELERATOR LABORATORY}

Batavia, Illinois

Scope of Work

This group directs its effort of research and development towards. the main ring of the Fermilab accelerator. Recent work includes making the accelerator operate in many different modes. For : example, 500-GeV operation, 2-Second flattop operation at $400 \mathrm{GeV}$ dc operation up to $200 \mathrm{GeV}$, and removal of rf structure on extracted beam. Work has also been done on the electrical feeder system and capacitor "tree" to improve cycle time. Addition of. 128 megahertz mode dampers to the $18 \mathrm{rf}$ cavities has Improved beam stability.

Goals and/or Major Results to Date

Major research and development will be directed toward increasing the intensity of the accelerator. Studies are beginning on the feasibility of multiturn injection into the main ring. The goal is to achieve greater than $5 \times 10^{13}$ protons per pulse. Some effort will also be used to work on reversing the direction of the protons in the main ring. This will be necessary for future colliding beam facilities.

$$
* * * * * * * * * * * * *
$$

Title of Project: Mechanical Support

Person in Charge: M.L. Palmer

Scientific and Professional Staff: R. Andrews, D. Cosgrove, E. Gallagher, G. Lee, M. May, L. Sauer, T. Peterson.

\section{Scope of Work}

This group directe its effort of maintenance and development towards the mechanical problems of the accelerator. Recent major efforts include installation of prototype doubler magnets into. the main ring tunnel, design of the negative ion vacuum box with remote controls for moving magnets, modification of the main series capacitor bank for better reliability, design work on the helium satellite refrigerator system for the switchyard, design, and fabrication work on collimators, patient chair and shielding for the Cancer Therapy. Facility, plus the normal maintenance and instaliation lin the accelerator. 
Goals and/or Major Results to Date

During the next year the principle areas of work will be on the negative ion injection system, two-turn injection system for the main ring, switchyard development of a cryogenic magnet system for use with the doubler, installation of Energy Saver/Doubler magnets in the main ring tunnel, design and development of the magnet line for reversing the main ring, and design of a new experimental area for colliding beams.

$$
* * * * * * * * * * * * *
$$

Title of P̈roject: Accelerator Operations

Person in Charge: H.E. Fisk.

Scientific and Professional Staff: J. Gannon, K. Meisner, G. Urban, R. Webber

\section{Scope of Work}

The Accelerator Operations group has as its primary responsibility the operation of the accelerator systems on a 24-hour basis. This work requires an operating crew of five people on shift who are responsible for learning and carrying out the operating procedures for the preaccelerator (Cockcroft-Walton, $750 \mathrm{keV}$ ), 1inear acce1erator $(200 \mathrm{MeV})$, booster $(8.8 \mathrm{GeV})$, main ring $(400 \mathrm{GeV})$ and extracted beam lines. The group contributes to instrumentation, programming and safety efforts which are aimed at running the machine in a more productive way. The professional members of the group participate in the accelerator research and development programs.

The accelerator is normally scheduled to operate an average of 150 hours per week. Recent improvements in the accelerator systems and operations procedures has permitted an average of 120 hours per week with 105 hours used for high energy physics research and 15 hours for accelerator research. Scheduled maintenance and development amount to 12 hours per week and startup and accelerator failure account for 36 hours per week. The average intensity at $400 \mathrm{GeV}$ is approximately $1.5 \times 10^{13}$ protons per pulse.

Goals and/or Major Results to Date

Goals include $3 \times 10^{13}$ protons per pulse, and a failure plus startup time not to exceed 24 hours per week.

$$
* * * * * * * * * * * * *
$$




\section{FERMI NATIONAL ACCELERATOR LABORATORY \\ Batavia, Illinois}

Title of Project: Superconductor

Person in Charge: R.R. Wilson

Scientific and Professional Staff: J.E. Finks, R. Flora, W.B. Fowler,

K. Koepkè, M. Kuchnir, P. Limon, R. Norton, P.V. Livdah1, R. Peters,

C. Rode, B.P. Strauss, A. Tollestrup, R. Walker

Scope of Work

This group directs its effort towards the construction of the Energy Saver/Doubler accelerator. Recent work has produced a 22 -foot prototype, 45 kilogauss superconducting magnet that is approaching a final design for the accelerator. The accelerator. will require 750 of these dipoles. Work is beginning on a central helium liquifier plant capable of producing 2500 liters per hour of liquid helium.

\section{Goals and/or Major Results to Date}

The next goals will be to install the first 100 dipoles in the main ring tunine1; develop a quadrupole (250) for the accelerator; develop an $r f$ accelerating cavity for the Energy Saver/Doubler; design and install the controls; and modification of the main ring power supply for the superconducting magnets.

A significant goal is to install all the magnets in the tunnel and. to obtain a coasting beam. If that beam, or the beam in the main ring can be reversed, there is the exciting possibility of bringing them into collision.

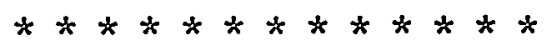




\section{LAWRENCE BERKELEY LABORATORY \\ Berkeley, California.}

Contract No. W-7405-ENG-48

Title of Project: PEP (Positron-Electron Project)

Person in Charge: T. Elioff

Scientific and Professional Staff: B. Austin, R. Avery, R. Belshe, E. Berget, M. Donald, T. Elioff, G. Eno, A. Faltens, A. Garren, J. Gunn, W. Harrison, E. Hartwig, T. Jackson, A. Lake, L. Laslett, V. More, P. Oddone, J. Peterson, C. Pike, D. Reimers; D. Rondeau, R. Sah, L. Smith, J. Tanabe.

\section{Scupe of Work}

The PEP project is a joint effure with SLAC. The project consists of the design and construction of a positron-electron colliding beam facility with energy up to $18 \mathrm{GeV}$ per beam and with a peak Iuminosity of $10^{32} \mathrm{~cm}^{-2} \mathrm{sec}^{-1}$. The scope of work includes theoretical activities on storage ring design and colliding beam phenomena and the development of devices and components for this new generation storage ring. Technical developments include, but are not limited to, injection systems, beam transport elements, high field quality quadrupoles, magnet design, advanced survey and alignment techniques, power supply systems, instrumentation and control systems, computer program development, and radio frequency systems. Also included is the development of experimental facilities, buildings and structures, and overall power and utilities for the project.

Goals and/or Major Results to Date

The conceptual design report for the PEP project was completed in February 1976. Englneering design on many technical components was begun in May 1976. An architectural-engineering firm has been selected for the design of conventional facilities and contractural negotiations are in progress. The overall project is on schedule with completion projected for early 1980.

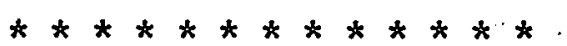

Title of Project: ESCAR (Experiuntal superconducting Accelerator Ring)

Person in Charge: E. Lofgren, G. Lambertson

Scientific and Professional Staff: R. Acker, R. Byrns, W. Chupp, W. Eaton, A. Faltens, A. Garren, W. Gilbert, E. Hoyer, G. 


\section{LAWRENCE BERKELEY LABORATORY}

Berkeley, California

Lambertson, L. Laslett, E. Lofgren, K. Lou, B. Main, R. Meuser, J. Peterson, C. Pike, J. Rechen, J. Staples, L. Smith, F. Toby,

F. Voelker, H. Vogel, R. Warren, J. West, D. Wolgast, R. Warren

Scope of Work

The ESCAR project will design, build, and operate a small-scale accelerator and storage.ring that is based on superconducting magnets and cryogenic technology. As the first superconducting accelerator, the machine will be a test facility for accelerator science and technology, intended to provide experience and data that is applicable and needed in planning the inevitable largescale use of the new technologies in accelerators and beam handling systems. Emphas1s is being placed on the adaptations and economic design of complete systems that will be affected by the superconducting components and the cryogenic environment. Because of its experimental: nature and comparatively small size, the project can pursue and evaluate untried features such as (1) the use of cold walls in the vacuum of a storage ring, (2) economical schemes for helium distribution, or (3) instrumentation suitable for new machines: : The capability of accelerating beam is needed to make such a project realistic in detail and the completed ring with beam is then available for needed experiments in accelerator science: The ring will be located at the end of the experimental hall at the Bevatron, available to protons from the Bevatron injector: and to heavy ions from the Hilac.

Major parameters of ESCAR are:

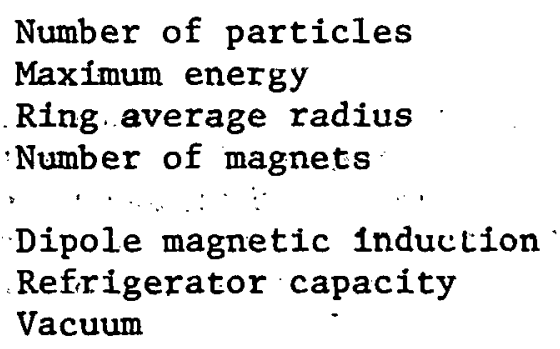

$4 \times 10^{12}$ protons.
$4.2 \mathrm{GeV}$
15.3 meters
24 dipoles
32 quadrupoles
4.6 tesla
1500 watts at $4.2 \mathrm{~K}$
$10^{-11}$ Torr

Goals and/or Major Results to Date

The fabrication and assembly of ESCAR is proceeding at a rate projected to complete the ring in FY 1980. In view of the need for relevant data on new accelerator systems, the project will proceed as rapidly as possible within funding limitations. At this time, the prototype dipole and its cryostat have performed satisfactorily and the fabrication and testing of the production units is underway. Housing for the large refrigerator is complete, the compressors are being 


\section{LAWRENCE BERKELEY LABORATORY}

\section{Berkeley, California}

installed, and the cold box is on order. First major tests of the partial systems will be conducted in 1977 when one half of the dipoles and cryostats will be installed at the final site, along with refrigerator, cryodistribution, power supplies, and controls. Beam experiments for ESCAR such as antiproton cooling for particle physics or beam dynamics tests for a fusion pellet igniter are under discussion.

$$
\text { * * * } * * * * * * * * * \star *
$$

Title of Project: Superconducting Magnets

Person in Charge: E. Lofgren, W. Gilbert

Scientific and Professlonal Staff: R. Acker, W. Chupp, W. Eaton, W. Gilberc, K. Meuser, F. Toby

Scope of Work

The Superconducting Magnet group concentrates on the application of superconducting magnet technology to high energy physics, primarily in accelerator ring and beam lines and, more recently, in large particle detector systems. Materials testing is fundamental to our research program which is directed to developing magnet systemsthat are reliable and predictable in performance. New magnet applications for fusion or other energy related fields are a natural outgrowth from the saddle shaped coils already developed.

Goals and/or Major Results to Date

A $60 \mathrm{kG}, 3$ foot long solenoid was used in a physics experiment at the $184^{\prime \prime}$ cyclotron in 1968.

A beam 1ine consisting of a 3 foot long $40 \mathrm{~kg}$ dipole and 2 - 2 foot long quadrupoles was run at the Bevatron in 1973, the longest continuous run being approximately 3000 hours ( 17 weeks). This beam line is now operating at the Fermilab Internal Target Area.

The ESCAR magnets, materials, and methods of construction were largely developed by members of the Supercon group. This has bccn our major activity for the past two years.

Various materials and cryogenic assistance has been provided to the Physics Division large solenoid detector development program

$$
* * * * * * * * * * * * *
$$


LAWRENCE BERKELEY LABORATORY

Berkeley, California

Title of Project: Research on $\mathrm{e}^{+} \mathrm{e}^{-}$Annihilation at SPEAR

Person In Charge: W. Chinowsky, G. Goldhaber, G. Triling

Sclentific and Professional staff: G. Abrams, W. Chinowsky, R. DeVoe,

C. Friedberg, G. Goldhaber, J. Kadyk, R. Madaras, K. Nguyen, F. Plerre,

B. Sadoulet, S. Shannon, G. Trilling

Scope of Work

The project is concerned with the study of $\mathrm{e}^{+} \mathrm{e}^{-}$annihilation reactions. These studies led to the discovery of the $\psi$ particles, $\psi(3.1)$ and $\psi(3.7)$. Three additional levels have been found between these. They are located at $3.41,3.5$, and $3.53 \mathrm{GeV}$. The second is a level which was first observed at DESY. Furthermore there are at least two additional resonances above the $\psi(3.7)$ which are relatively broad. These are at 4.15 and $4.4 \mathrm{GeV}$.

The $\psi$ particles described above provide evidence for the existence of a new particle property called charm. The $\psi^{\prime} s$ do not themselves exhibit this property; its existence is only made plausible by their discovery.

Demonstration of the existence of charm requires the production of charmed particles. These, according to theory, decay with high probability into a strange particle and ordinary companions.

Goals and/or Major Results to Date

Recently the LBL-SLAC team has found a pair of particles with this property. They are ${ }^{a}{ }_{ \pm}$neutral and $a_{\mp}$ changed boson, $D^{0}, D^{+}$which decay as $D^{O} \rightarrow k^{ \pm} \pi^{\ddagger}$ or $k^{ \pm} \pi^{ \pm} \pi_{\pi}$ and $D^{+} \rightarrow k^{\mp} \pi$. The $D^{+}$does not decay into $\mathrm{k}^{\mp} \pi^{+} \pi^{-}$in accordance with charm theory which predicts the exotic decay channe1.

The SPEAR colliding $\mathrm{e}^{+} \mathrm{e}^{-}$beam machine has the remarkable property of transversely polarizing the electron and positron beams. At a total energy of $7.4 \mathrm{GeV}$ anisotrophy in the distribution of hadron prongs as a function of the azimuthal angle $\Psi$ has been observed. The distributions are indicative of the polarization expected for spin $1 / 2$ initial states in agreement with expectations from the parton model. Furthermore, it was observed that at this energy the hadron distributions are no longer consistent with phase space but rather showed marked "jet" characteristics. This means that for given events we find a jet axis with respect to which the hadrons distribute themselves with low transverse momentum. This feature is again in accord with the parton model.

$$
\text { *************** }
$$




\section{LAWRENCE BERKELEY LABORATORY \\ Berkeley, California}

Title of Project: Time Projection Chamber

Person in Charge: D. Nygren

Scientific and Professional staff: 0. Dahl, P.. Eberhard, D. Fancher, R. Kenney, S. Loken, G. Lynch, J. Marx, D. Nygren, P. Oddone, M. Pripste1n, P. Robrish, G. Shapiro, M. Stevenson, M. Strovink, M. Urban, W. Wenzel

\section{Scope of Work}

The time projection cliamber is a charged particle detector for PEP which will allow viewing of the entire $4 \pi$ of solid angle around an Interaction region. This device Incorporates new ideas in drift. chamber technology such as suppression of electron diffusion by magnetic flelds and detection techniques which provide intrinsically 3-dimensional data. Such a particle detector wili offer dramatic improvement in the area of pattern recognition in high-multiplicity events. The basic advantages of this detector concept resuit from the configuration of parallel magnetic and electric fields.. Track images (the lonization electrons left behind by.a high energy particle) are drifted through an argon-methane mixture to a plane of wires which give both a measurement of $\mathrm{dE} / \mathrm{dx}$ through pulse-height information and localization of the trajectories through induced pulses on a segmented cathode plane. The resolution of $\mathrm{dE} / \mathrm{dx}$ will allow particle mass Identification, even in the relativistic region. The sensitive solid angle of the device will be about $90 \%$ of $4 \pi$. Momentum resolution w111 be $1 \%$.

Goals and Major Results to Date

Lmportant time projection chamber studies completed this year include:

1) Extensive high accuracy measurements of transverse diffusion of electrons in varfous mixtures of argon-methane.

2) Experimental determination of the effects of nonparallelism of the magnetic and electric fields.

3) An evaluation of alternative readout schemes which has led to the conclusion that the optimum geometry is a plane of proportional wires with a segmented cathode plane.

Currently, measurements of $\mathrm{dE} / \mathrm{dx}$ are in progress at the Bevatron. These data will allow optimization of a large-scale prototype time projection chamber as well as new infurmacion about energy loss in extremely thin absorbers.

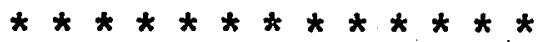




\section{LAWRENCE BERKELEY LABORATORY \\ Berkeley, Calffornia}

Title of Project: Lead Glass Wall

Person in Charge: A. Galtieri

Sclentiflc and Professional Staff: R. Ely, A. Galtier1, P. Lecomte, R. Madaras, T. Mast, M. Ronan, R. Ross, V. Vulllemin

Scope of Work

The lead glass wall is a particle detector which is sensitive to photons and electrons. The apparatus consists of a combination of lead glass shower detectors and track chambers to be attached to the outside of the SPEAR magnetic detector. The device w111 be used to study anomalous electron production and production of photons and $\pi^{\circ} s$ in $e^{+} e^{-}$annihilation. The primary aim is to search for events which might arise from the production and leptonic or semi-leptonic decays of new particles (charmed particles, heavy leptons, or any new objects). The apparatus can also measure inclusive production of : $\gamma$ rays above $100 \mathrm{MeV}$ and $\pi^{\circ} / 8$ above $500 \mathrm{MeV} / \mathrm{c}$.

The responsibility for building the apparatus has been divided between LBL and SLAC. The LBL part of the collaboration is responsible for building the frame containing the lead glass as well as the lead glass counters themselves with the assoclated electronics and monitoring system.

Goals and/or Major Results to Date

A serles of tests have been carried out at the Mark III accelerator at Stanford and at SLAC. Tests to determine the optimum glass type and photomultipliers for lead glass Cerenkov counters were done at the Mark III. In addition the energy resolutions of a vartety of different counter geometries were measured. A fraction of the final "lead glass wall" has been studied in an electron and pion beam at SLAC in order to test all the components as well as to study the e/ $\pi$ separation capabilities of the system. It is designed so as to achleve a $\pi /$ e rejection of $2 \times 10^{-3}$ at $1 \mathrm{GeV} \pi / \mathrm{e}$ energy. The components we have tested so far are:

1) Lead glass counters,

2) LED system for monitoring the photo-tube gains to a few percent,

3) The adders (electronic devices to add the energy deposited in a row of counters) which are part of the trigger system.

The automatic digital converters for the data acquisition, designed and bullt at LBL, w11l soon be tested.

*************** 


\section{LAWRENCE BERKELEY LABORATORY \\ Berkeley, California}

Title of Project: Counter Experiments on $k^{-} p$ and $\bar{p} p$ Reactions at BNL

Person in Charge: R. Tripp

Sclentiflc and Professional Staff: M. Alston-Garnjost, R. Kenney, R. Ross, R. Tripp

Scope of Work

This project is concerned with a detalled study of $k^{-} \mathrm{p}$ and $\overline{\mathrm{p} p}$ rcactions. The multi-channel character $\left(k^{\circ} n, \Lambda \pi, \Sigma \pi\right)$ of the $k^{-} p$ reaction is of considerable interest oo it hus been studied for this reason as well as the search for baryon resonances. Studies of the $\bar{p}$ reaction give information regarding theoretiral modelo which suggest the existeuce of resonanccs in the region 0.3 to 1.0 $\mathrm{GeV} / \mathrm{c}$.

Goals and/or Major Results to Date

Experiments on the cross section for $k^{-} p \rightarrow k^{0} n$, in the region 0.5 to $1.1 \mathrm{GeV} / \mathrm{c}$, have been completed. Measurements of the angular distribution have also been made and the results are being analyzed. The $\bar{p}$ p total cross section has been determined in the momentum region 0.3 to $1.0 \mathrm{GeV} / \mathrm{c}$ but no resonance has been found.

A new experiment, BNL 691, on $k^{-} \mathrm{p}$ backward elastic scattering has been approved and will soon be underway.

$$
\text { *************** }
$$

Title of Project: Particle Data Center

Person in Charge: A. Rittenberg

Scientific and Professional Staff: C. Horne, R. Kelly, A. Rittenberg, T. Trippe, G. Yoot

Scope of Work

The Particle nata Center is involved in four major activites:

1) Compilation of particle properties,

2) Compilation of cross sections, angular distributions, density matrix elements, etc., 


\section{LAWRENCE BERKELEY IABORATORY \\ Berkeley, California}

3) Compilation of bibllographic information and experiment description (beam, target, momentum, reactions, etc.) for all approved accelerator proposals, and

4) Development of a computerized data base management system to store, malntalin, update, retrleve, and display the compiled information.

Goals and/or Major Results to Date

Projects presentiy underway include:

1) A general purpose data base management system (BKY-DBMS),

2). The application of BKY-DBMS to the management of our "Part1cle Physics Document File,"

3) Work is also proceeding on the "Particle Physics Reaction-Date File," which will eventually contain the actual data on reactions, extracted from our particle physics documents,

4) A $\pi N$ data compilation, data amalgamation, and partial-wave analysis project has continued, and

5) The IRATE/OTRIR text entry and editing system has continued to develop. The system is now capable of producing complex, sclentific text and tables such as might be found in a journal article.

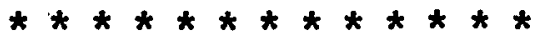

Title of Project: Superconducting Magnets

Person in Charge: P. Eberhard

Sclentific and Professional Staff: P. Eberhard, M. Green, J. Taylor, V. Vulllemin, W. Wenzel

Scope of Work

There is a class of detectors that w1ll be used around storage ring accelerators and will require the use of superconducting solenotds with high current density. Inside of the coll, the momenta of the charged particles will be measured, using magnetic fields of 1.0 to 2.0 tesla and, outside of the coll, additional information will be collected by other detectors (Cerenkov, shower counters, etc.) about charged or neutral particles that have traversed the coll. Diameters of 1 to $2 \mathrm{~m}$ are typically considered. Two types of magnets are 


\section{LAWRENCE BERKELEY LABORATORY \\ Berkeley, California}

considered: a) The continuous solenold with a minimum thickness to eliminate absorption and electromagnet1c showering. b) The lumped coll magnet with the coils subtending the minimum possible solid angle around the beam intersection.

\section{Goals and/or Major Results to Date}

Studies of different magnet conflgurations have been performed during 1975 and 1976. The design of a continuous solenold of $1 \mathrm{~m}$ in diameter, $1 / 3$ of a radiation length in thickness and consisting of four Identical modules has been nuade. Two such modules have been built and tested to the full design current, $700 \mathrm{~A}$. For this test, an original testing method has been used and the properties of quenches have been studied to help improve future designs.

$$
\star \star \star * * * * * * * * * * *
$$

Title of Project: Primordial Black-Body Radiation

Person in Charge: R. Muller

Scientific and Professional Staff: L. Alvarez, R. Muller, G. Smoot

Scope of Work

The project to detect and map large scale anisotropy in the $3^{\circ} \mathrm{K}$ primordial black body radiation is continuing. If the earth moves relative to a frame fixed with respect to the ancient and distant matter of the universe in which the average energy is $\mathrm{kT}$, then in an earth frame this energy will be $\mathrm{kT}^{\prime}=\mathrm{kT}(1+(\mathrm{v} / \mathrm{c}) \cos \theta)$, with $v$ the earth's speed. With an angular resolution of $15^{\circ}$ and a sensitivity of $2 \times 10^{-4} \mathrm{~K}$ existing measurements will be improved by a factor between 10 and 20. The earth's motion being studied is due to the rotation of the Milky-Way galaxy, as well as the orbit of the earth around the sun. We will detect possible rotation of the Universe (with respect to local inertial frames) with sufficient sensitivity to see less than $10^{-3}$ radians per age of the Universe (about $10^{10}$ years). In addition we expect to detect anisotropies from irregularities in the primordial plasma which must have been present if current theorles of galaxy formation are corruct. Radiometers w11I be flown in U2 aircraft at 65,000 feet.

Goals and/or Major Results to Date

Construction of experimental apparatus has been completed, and Lockheed has finished modifying the U-2 upper hatch to fit our experiment. The two radiometers and their antennas have been 


\section{LAWRENCE BERKELEY LABORATORY \\ Berkeley, California}

Installed in the modifled U-2 upper hatch and tested in regard to its nolse figure, radio frequency interference shielding and ground loop pickup. The equipment has checked out in a daytime "piggy-back" flight, performed simultaneously. with other U-2 earth resources, missions, and further flights are scheduled.

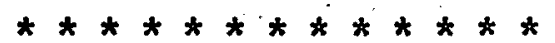

Title or Project: Rubber Mirror

Person in Charge: A. Buffington

Sclentiflc and Professional Staff: A. Buffington, F. Crawford, R. Muller, C. Orth.

Scope of Work

The resolving power of optical telescopes with apertures greater than. $10 \mathrm{~cm}$ has been IImited by atmospheric distortion rather than by diffraction ever since the first astronomical telescopes were built hundreds of years ago. The present project is intended to eliminate atmospheric distortion in ground-based telescopes through use of a feed-back scheme and an optical element (rubber mirror) which can compensate for the distortions introduced by the atmosphere. The theory of this feedback scheme has been worked out and indicates that the system should work for celestial objects brighter than about 12 th magnitude. The scheme attempts to maximize the "image sharpness" which can be defined as "I ${ }^{2}$, where $I$ is the intensity of the Image as a function of the coordinates in the image plane. The system can be conveniently employed on existing telescopes, and is presently designed for use on a 30-1nch telescope maintained near Berkeley by the University of California Astronomy Department.:

\section{Goals and/or Major Results to Date}

In spite of a prevalence of bad weather the system has been used to make an image of Sirius whose full width at half maximum is $1 / 3$ a second of arc. Th1s is the best resolution that has been achieved in astronomy.

$$
* * * * * * * * * * * * *
$$

Title of Project: Optical Pulsars

Person in Charge: J. Nelson

Scientific and Professional Staff: J. Middleditch, J. Nelson 


\section{LAWRENCE BERKELEY LABORATORY \\ Berkeley, California}

Scope of Work

Work on fast photometry, and the study of the optlcal behavior of compact astronomical objects (white dwarfs, neutron stars, black holes) has continued. Fast photometry deals with the measurement of the visible behavior of astronomical objects on a short timescale; seconds or less. This techinique is well sulted to study the rapid varlations in luminosity seen in compact objects. It has been made practical only through relatively new and sophisticated methods for evaluating Fourier transforms.

Goals and/or Major Results to Date

B. In 1975 a major phase of the study of the optical pulsations of the $X$-ray pulsar Hercules $\mathrm{X}-1-\mathrm{HZ}$ Herculis was completed. By carefully analyzing the apparent frequency of optical pulsatiuns and comparing these with the $X$-ray frequency the masses of both stars in this binary system have been found. In particular the first accurate measurement of the mass of a neutron star $\left(M_{H e r} X-1=1.3 M_{0}\right)$ has been
made.

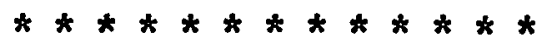

Title of Project: Mark II Magnetic Detector

Person in Charge: W. Chinowsky, G. Goldhaber, f. Tri11ing

Scientific and Professional Staff: G. Abrams, W. Car1thers, W. Chinowsky, R. DeVoe, G. Goldhaber, J. Kadyk, S. Shannon, G. Tr1111ng

Scope of Work

The Mark II detector incorporates a large solenoldal magnetic fleld geometry and cylinderical arrays of particle detectors for momentum determination and identification of secondary particles produced in $\mathrm{e}^{t} \mathrm{e}^{-}$interactions. The Mark II, although simflar to the SPEAR MKI magnetic detector, uses several new technologies to improve solid angle coverage, momentum resolution, neutral particle detection, spatial resolution and energy resolution.

Goals and/or Major Regults Lu Date

Design studies for the Mark II magnetic detector scheduled for installation at SPEAR in 1977 have continued. A small model of the Mark II system was tested at SLAC and the results obtained for energy resolution are consistent with expectations based on shower development theory. The lead-1iquid argon shower counter detection 


\section{LAWRENCE BERKELEY LABORATORY \\ Berkeley, California}

system which is planned will yield both energy measurements and position information on electron and gamma ray showers, as well as pl-electron separation. Design studles for the full-size shower counter modules, which will completely surround the three-meter dlameter solenoidal magnet, are in progress.

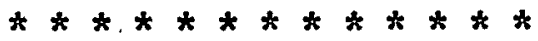

Title of Project: Neutrino Interactions

Person in Charge: M. Stevenson

Scientific and Professional Staff: P. Bosett1, A. Galtier1, G. Lynch, F. Solmitz, M. Stevenson

Scope of Work

Neutrino interactions in the Fermilab 15-foot bubble chamber have been studied with the chamber filled with hydrogen and with a mixture of $23 \%$ neon and hydrogen. The Important neutrino physics; topics studied thus include deep inelastic interactions, diffractive efastic production, single particle hadron inclusive distributions, $\Delta$ production, inclusive neutral current production, strange particie production, and search for exotic states.

Goals and/or Major Results to Date

Evidence for the existence of a particle with entirely new physical properties was found in scanning a portion of the 80,000 pictures taken with the neon hydrogen mixture. Events of the type $e^{+} \mu k^{-}$ were observed that has the property of "quantum number" that may correspond to the one called charm predicted by theorists several years ago. This is an Hawa11-LBL-Wisconsin collaboration.

In the study of neutrino interactions using intense beams of muon neutrinos such as are produced at Ferml National Accelerator Laboratory, muon identification is of prime importance. The External Muon Identifier was concelved to ald in the study of neutrino interactions of the type: $\nu+p \rightarrow \mu^{-}+$hadrons. In such Interactions, the lepton changes from neutral $(v)$ to charged $\left(\mu^{-}\right)$ and delivers a "charged current" to the inltial hadron, the proton. To analyze this type of event kinematically and compare it with theoretical models, the outgoing muon must be distinguished from the hadron tracks in the bubble chamber. The External Muon Identifier now In operation at FNAL, consists of a set of 24 multi-wire proportional chambers placed behind the 15-foot bubble chamber. The device was developed by LBL and the Univeristy of 


\section{LAWRENCE BERKELEY LABORATORY \\ Berkeley, Callfornla}

Hawa11, and constructed with FNAL support at LBL. It has been operational for all neutrino and antineutrino runs with the 15-foot chamber.

FNAI has approved plans for LBL and the University of Hawaii to improve the EMI. These include the construction of more chambers to provide double chamber coverage for at least part of the area, addition of one more channel of readout on the chambers, construction of new digitizers that will allow operation at higher data rates, and construction of a "plcket fence" array of counters in the bubble chamber vasuum to provide a prompt time slynal for an event, so as to effectively reduce the background.

An active program has been underway at LBL to develop and Improve computer programs for the analysis of the data from the 15-foot chamber EMI hybrid system.

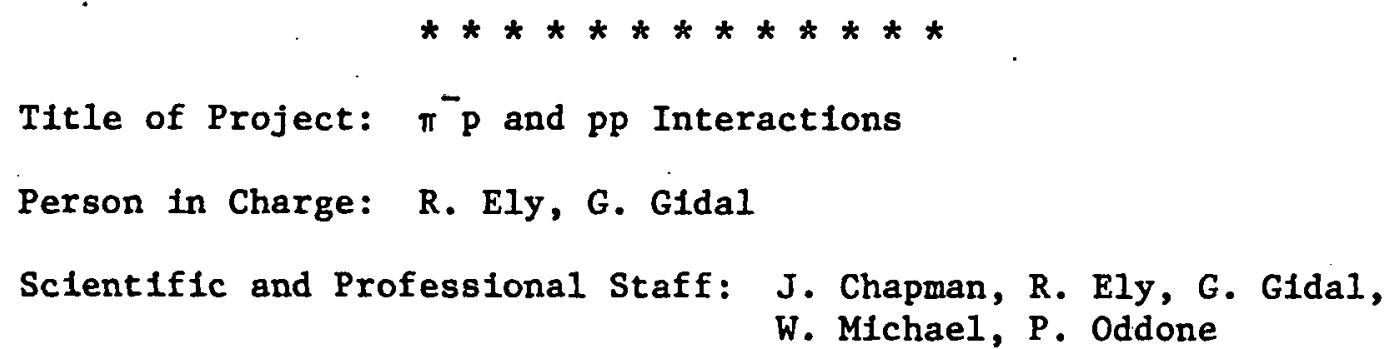

Scope of Work

In collaboration with groups from the California Institute of Technulogy and Stanford Linear Accelerator Eenter, LBL physicists are studying the diffractive production and decay of nucleon isobars produced by $14 \mathrm{GeV} / \mathrm{c}$ pions. Counters and wire spark chambers are used to trigger the SLAC 40-inch $\mathrm{H}_{2}$ bubble chamber on pictures with a fast outgoing track of more than $8 \mathrm{GeV} / \mathrm{c}$. The external measurements of the fast forward track allow both an efficient trigger and increased resolution. 400,000 pictures with incident $\pi^{-}$and 600,000 pictures with incident $\pi$ have been taken.

In collaboration with U.C. Davis an exploratory program has been undertaken in the 15-foot bubble chamber at FNAL to study pp interactions at high energies. The first phase on this program is to develop scanning and measuring techniques and to develop reconstruction programs. The physics objectives of this experiment include a search for charm, a direct measurement of neutral-charged particle correlations, inclusive cross sections for the production of vector mesons and $\Delta(1238)$, and a study of hypercharge transfer contributions. 


\section{LAWRENCE BERKELEY LABORATORY}

Berkeley;, California

Goals and/or Major Results to Date

In late December 1975 a data run of some 29,000 pictures was completed using the Fermilab 15-foot bubble chamber filled with liquid hydrogen and exposed to a beam of $400 \mathrm{GeV} / \mathrm{c}$ protons. Event reconstruction programs have been tested and successfully used to reconstruct events from this film. Prelfminary scanning and measurement are now underway while further refinements are made to the analysis chatn.

An experiment on baryon exchange reactions is designed to study exchange mechanisms in backward $\pi p$ inelastic scattering around $4 \mathrm{GeV} / \mathrm{c}$. A large aperture Cerenkov counter and hodoscope were used to trigger the LBL streamer chamber on interactions producing a fast forward proton or $\mathrm{K}^{+}$. Picture taking has been completed and analysis is well underway. Approximately 70,000 events representing $-2 / 3$ of the available data have been measured on the COBWEB system. Programs have been developed to Incorporate beam and downstream spark chamber measurements into the streamer chamber measurements, signiflcantly improving the resolution over the bare streamer chamber measurements. The following aspects of $\pi^{-} p$ inelastic scattering at small u values are being studied:

1) Search for backward $\mathrm{A} 1$ and $\mathrm{A} 2$ production by both $\mathrm{N}$ and $\Delta$ exchange.

2) Backward vector meson production and decay; $\pi^{-} p \rightarrow p \bar{p}$, $\pi \mathrm{p} \rightarrow \mathrm{N}^{*} \mathrm{O}_{\mathrm{\rho}} \mathrm{O}, \pi^{-} \mathrm{p} \rightarrow \mathrm{N}^{* 0} \omega^{0}$, etc. Final statistics will allow a study of each of these reactions at the 500-1000 events level. Comparisons can be made with reactions in which a $\rho$ and $\omega$ are exchanged. The $\rho^{\circ}-\omega^{\circ}$ interference in this backward production is seen.

3) Backwald nueleon leobar prnduction and cascading $\mathrm{N}^{*}$ decays.

4) Approximately 5\% of the proton triggers came from the decay of $\Lambda^{\circ} \mathrm{s}$. This allows the study of the reaction $\pi p \rightarrow \Lambda^{\circ} K^{*}$. In detail. Based on approximately $1 / 4$ of the final data, the polarization of the $\Lambda^{\circ}$ at small $u$ shows a dip near $u=-0.15$.

5) Search for exotic mesons.

$$
\text { *************** }
$$




\section{LAWRENCE BERKELEY LABORATORY \\ Berkeley, California}

Title of Project: Polarization Studies in $\pi^{-} p, k^{-} p, \bar{p} p$ Scattering

Person in Charge: 0 . Chamberlain

Scientific and Professional Staff: W. Bruckner, 0. Chamberlain, G. Shapiro, H. Steiner

Scope of Work

This experiment at Fermilab measures the polarization parameter in high energy $\pi^{ \pm} \mathrm{p}, \mathrm{K}^{ \pm} \mathrm{p}$, and $\overline{\mathrm{p} p}$ scatlering at $\mathrm{L} \cup U \mathrm{GeV}$ in the region of $t=-0.15$ to $t=-1.5 \mathrm{GeV} / \mathrm{c}^{2}$. This experiment is a collaborative effort Involving physicists from Harvard, Yale, Argonne Nattonal

Laboratory, Fermilab, and LBL.

Goals and/or Major Results to Date

Successful initial runs have been completed and analysis of the data is in progress. Less than $10 \%$ assymmetry has been observed in accord with a model based on pomeron and $p$ exchange.

Future work will extend to higher (negative) values of $t$ and $a$ longltudinally polarized beam will be used to study the spin rotation parameter. Additional equipment, especially another very large PWC is needed before the Wolfenstein parameter $D$ can also be measured, simultaneously with the polarization $P$. The large PWC is being constructed.

$$
* * \star * * \star \star \star \star \star \star \star * * * *
$$

Title of Project: Experiments on $\pi^{-}$p Reactions at FNAL

Person In Charge: R. Kenney

Scientiflc and Professional Staff: 0 . Dahl, R. Johnson, R. Renney, M. Pripstein, S. Shannon

Scope of Work

This experiment is to investigate the following $\pi^{-}$p_reactions in the momentum range 20 to $200 \mathrm{GeV} / \mathrm{c}:$ if $\left.\left.\pi^{-} p \rightarrow \pi n, 2\right) \pi p+n n, 3\right)$ $\pi^{-} p \rightarrow \pi^{0}+$ anything and 4) $\pi^{-} \rightarrow \pi^{6}+$ neutrals. Three different investigations are possible by altering the configuration of the particle detectors. These are a) an investigation of Regge trajectories, b) studies of mode1s for production mechanisms, and c) a test of the triple-Regge formallsm inclusive cross sections. The first two $\pi^{-} p$ reactions are classic from the point of view of Regge theory since each 


\section{LAWRENCE BERKELEY LABORATORY \\ Berkeley, California}

Is dominated by the exchange of a single Regge trafectory, the $\rho$ and the $A_{2}$, respectively. Studies of these same reactions at large transverse momenta provides the first detalled comparison between these reactions at high energies and should yield significant information about the possible quark or parton of the particles involved in the reactions. The triple-Regge formalism has been successful in fitting the pp inclusive cross sections, but since there are so many parameters this is not a very conclusive test. The corresponding prediction for reaction 3) is simple by comparison and measurement of this inclusive cross section should provide a definitive test of the theory.

\section{Goals and/or Major Results to Date}

The $\pi^{-}$p program at Fermilab has investigated $\pi^{-} p \rightarrow \pi^{0} n$ and $\pi^{-} p \rightarrow n n$ reactions to $200 \mathrm{GeV}$ and for $|t|$ between 0 and 1.5 and found agreement with Regge theory for $p$ and $A_{2}$ trajectorles. At the largest $|t|$ early. hints have been found in this experiment of a non linearity in the Regge trajectory for the $\rho$. The $\pi^{-} p+w n$ reaction is accurately. predicted by Regge theory with natural parity exchange.

Comparisons of inelastic $\pi^{ \pm} p \rightarrow \pi^{\circ} \overline{\bar{X}}$ and $p p+\pi^{\circ} \underline{\bar{X}}$ reactions at 100 and $200 \mathrm{GeV}$ incident particle energy agree with simple quark scattering models, and clearly disagree with quark-antlquark fusion ideas. Three body final states are being analyzed.

The Triple Regge Region for $\pi^{-} p \rightarrow \pi^{0} \bar{x}$. Is currently under Investigation. This inelastic cross section is much larger than the elastic $\pi^{p} \rightarrow \pi^{\circ}$ : cross section and allows significant data to be collected at very large $|t|$. This reaction is dominated by the $\rho$. Preliminary extractions of the $\rho$ trajectory at values $|t|<5$ show that it asymptotes to -1 for $|t| \geq 3$, thus providing the first example of a non-linear Regge trajectory.

$$
\star \star \star * * * * * * * * * * *
$$

Title of Project: Rare Muon Reactions, Multi-Muon Spectrometer

Person in Charge: L.T. Kerth

Scientific and Professional Staff: A.R. Clark, L.T. Kerth, S.C. Loken, M.W. Strovink, and W.F. Wenze1

Scope of Work

The experiment depends on the ise of a 400-ton magnetic spectrometer located in the FNAL Muon laboratory. This serves both as the target 


\section{LAWRENCE BERKELEY LABORATORY \\ Berkeley, California}

material for the primary muon interaction and the analysis vehicle for secondary muons as well as hadron and electromagnetic showers. The design of the magnet features a transformer-type cross section for the 90 four-inch thick iron plates and excitation from a coil which loops all plates together. The field is a uniform $19 \mathrm{kG}$ over most of the active area of 3.5 foot $\times 6$ foot.

The spectrometer is arranged in 18 modules, each with 15 iron plates interspersed with calorimeter counters, which measure hadron energy with an accuracy, $\sigma(E)=0.9 \mathrm{E}^{-1 / 2}$ (energies in $\mathrm{GeV}$ ). At the downstream end of earh module thcre is a proportional counter-drift chamber system to locate precisely secondary muon tracks as well as the incident track upstream of the interaction. A trigger hodoscope for the single and multiple muon trigger also follows every second module. Proportional wire chambers provide the good time resolution and multi-track efficiency necessary to take data with instantaneous beam flux rates of up to $10 \% \mathrm{sec}$. The drift chamber in each module will provide high spatial resolution in the bending plane. This gives a momentum resolution which ranges from $7 \%$ to $11 \%$.

In order to carry out the study of rare modes the trigger counter and hadron calorimeter signals will be used together with beam signals and kinematic cuts to enhance the signal-to-noise ratio for final states with heavy muons, $\psi^{\prime}$ 's and other particles decaying into muons, as well as for deep inelastic events. For the latter the apparatus has acceptance up to $\mathrm{Q}^{2}+300(\mathrm{GeV} / \mathrm{c})^{2}$.

\section{Goals and Major Results to Date}

The maguel design was completed at LBL early in the year; Fermilab has approved the design and has solicited bids for the magnet materials and fabrication. Prototypes of the proportional chambers and of the associated electronics have been constructed and are under test. The calorimeter counters have been fabricated and are being tested. Time stability of photomultipliers is being studied extensively in order to improve the calorimeter performance. Monte Carlo studies have been carried out to improve the trigger efficiency; fabrication of the trigger counters will be completed this year. Effort has begun on development of both the on-line data acquisition programs and the of $f$-line analysis programs. This is a collaboration with FNAL and Princeton.

$* * * * * * * * * \star * \star * *$

Title of Project: Computer Center

Person in Charge: R. Harvey 


\section{LAWRENCE BERKELEY LABORATORY \\ Berkeley, California}

Scientific and Professional Staff: E.R. Beals, R.L. Fink, R.J. Harvey, J.A. Knight, P.A. Rhodes, and D.F. Stevens

Scope of Work

1) To provide large-scale (batch and Interactive) computational support to the scientific program of the Lawrence Berkeley Laboratory.

The nature of this support includes access to an extremely powerful computer (the CDC 7600), to a very large mass storage system $\left(3 \times 10^{11}\right.$ bits on-line), and to a wide range of peripheral devices. Large numbers of jobs (more than 980,000 in 1976) are processed, with excellent turnaround (about $70 \%$ were turned around in 20 minutes). A wide range of Interactive facilities is offered, including text editing, program preparation and submission, several interactive graphics subsystems, and the ability to interact with any job running on the front-end (6600 and 6400) computers.

LBL is also a mode on the ARPANET and supports both the interactive and the file-transfer protocols.

2) To provide the ancillary services necessary for convenient and effective use of a large-scale computing facility.

These services include a professional consultants' service, on-1ine user documentation of the entire system, convenient program preparation facilities, and a wide range of standard products and I1braries.

3) To make any excess capacity avallable to other ERDA laboratories and contractors.

Among the principal beneficiaries of this aspect of LBL's work are many university physics groups who find remote use of LBL's Computer Conter to be convenient. and economical.

Goals and/or Major Results to Date

Recent major achfevements of the LBL Computer Center Include:

1) The culmination of a performance-improvement program on the 7600 which provided a $20 \%$ increase in productivity, and led to a corresponding reduction in the charging rate.

2) The completion of the first CDC/IBM Channel Adapter (which w111 allow LBL, for the first time, to utilize IBM plus-compatible peripherals). 
LAWRENCE BERKELEY LABORATORY

Berkeley, California

Goals for improving and expanding Computer Center capabilities include:

1) To achieve the same excellence in interactive computing we have achieved in local and remote batch operations, without sacrificing batch performance.

2) To foster the LBL-SLAC PEP collaboration by developing a link between the laboratories as well as by providing the expected computing support.

3) To procure and Install a new mass storage device in a fashion transparent to the uscr. (The existing device is being removed from maintenance by the manufacturer.)

4) To become the site of the NRCC.

5) To maintain our strong1y service-oriented philosophy.

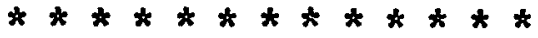

Title of Project: Instrumentation Development

Person in Charge: S.N. Kaplan, V. Perez-Mendex, and L.J. Wagner

Scientific and Professional Staff: S.N. Kaplan, F. Kirsten, P. LeComte, K.I. Lee, B. Leskovar, M. Nakamura, S.R. Olson, V. Perez-Mendez, R. Steele, and L.J. Wagner

Scope of Work

The instrumentation development group provides technical support of the high energy physics research program at LBL. Included among the latest instrumentation provided are mult1-wire proportional counters, micro-channel plate photo multipliers, and drift chambers. Th1s work includes all electronic circult and system development and fabrication to support these instruments. Much of the new instrumentation research carried out by the group has concentrated on developing special combinations of drift and mutli-wire proportlonal chambers with delay line readouts and to improving the properties of electromagnetic delay lines and their associated electronics (e.8., low noloc amplifiers and low slewing discriminators).

Goals and/or Major Results to Date

An investigation of micro-channel plate detectors with and without photocathodes is being made. Their insensitivity to magnetic fields makes them ideal for use in the presence of high magnetic fields-such as would be encountered in proposed PEP detectors. They are also 
important for ultra-fast time of flight measurements. A program to measure their resolving time and behavior in magnetic fields is underway.

The characteristics of a combined drift chamber with lead glass plates and delay-line readout for gama shower detection are under investigation.

Measurements on the timing resolution and position accuracy of low pressure parallel plate methane-filled chambers with avalanche multiplication have been carried out. This type of counter is expected to have a time resolution < $200 \mathrm{p} \mathrm{sec}$. By the addition of sense wires or by use of copper plated anodes on plastic, position resolution $<100$ microns are expected.

Development of new forms of delay lines, including metal plated on plastic, is continuing. For wire wound lines parameters have been varied in order to improve the delay to rise time ratio $(D / R>50$ at present). The goal is to obtain the narrowest possible two pulse resolution. The metal plated delay lines are for use in lead glass drift chambers and other applications requiring a multiplicity of delay lines per chamber.

Very low nolse amplifiers and very low amplitude slewing timing discriminators have been developed for use with drift-multi-wire proportional counters and for micro-channel plate particle position detectors.

A set of two high-accuracy cylindrical drift chambers of approximately $90 \mathrm{~cm}$ diameter and $150 \mathrm{~cm}$ length have been designed and are now under construction. In the meantime, tests have been performed with small chambers showing, in particular, that these can be operated in reglons where the electron drift velocity is saturated.

We have designed and fabricated an economical modular system for the digitization of analog data from hundreds of event channels simultaneously. The system digitizes various combinations of time, charge (pulse helght), and/or d.c. analog voltages to 10-bit resolution. In the time mode, multiplexing provides added economy and/or accommodates channels with multiple hits. Readout via the CAMAC standard data bussing system allows for discretionary readout of only channels which experienced "hits" during an event, or, alternatively, of all channels.

An economically attractive zero crossing discrimator has been developed for use with signals characteristic to multi-wire proportional chambers (MWPC's) readout by delay lines. Time walk of only \pm 100 ps for signals of $100 \mathrm{~ns}$ rise and fall time over a 30:1 amplitude range has been achieved with the development prototype unit.

$$
\text { ************** }
$$




\section{LAWRENCE BERKELEY LABORATORY \\ Berkeley, California}

Title of Project: Theoretical Physics

Person in Charge: J.D. Jackson

Sclentific and Professional Staff: K. Bardakci, M. Chanowitz, G.F. Chew, A A. Garren, M.B. Halpern, P. Hoyer, J.D. Jackson, D.L. Judd, L.J. Laslett, J.V. Lepore, S. Mandelstam, R.J. RIddell, Jr., L. Smith, H.P. Stapp, M. Suzuki, W.J. Swiatecki, K.M. Watson, W. Wilson

Scope of Work

Particle physics is the major research activity of the Theoretical Group although it is heavily committed to accelerator theory and design.

Research in particle theory is concerned with the strong, electromagnetic and weak interactions and covers the gamut from formal axiomatic field theory to phenomenological model building and analysis of data.

Most of the work on an approach to a dynamical theory of strongly interacting particles is based on the S-matrix concept which deals directly with experimental quantities. The broad outline of this theory has been clear for many years. It is based on the very general postulates that scattering amplitudes satisfy the requires ments of unftarity, analyticlty-except for predictable singularities, crossing relationships between particle and antiparticle processes and Lorentz invariance. In order to arrive at a well-defined theory these postulates are supplemented by the requirement of Regge behavior, 1.e., at very high energies amplitudes decrease as a power of the energy.

\section{Goals and/or Major Results to Date}

The aspects of an approach to strong interactions that has been called the "topological expansion" has been explored. The inverse expansion parameter, which measures the density of hadronic levels or particles, 1s, in certain regions, sufficiently large to allow a tractable calculational procedure based on unttarity. Three previously Independent strong-interaction models have emerged as different aspects of the topoluglcal expangion: 1) 'the wultipertpheral bootstrap model, 2) The quark-antiquark model of mesons with $\mathrm{Zwe} \mathrm{g}$ selection rules, and 3) Gribov's pomeron calculus.

Hadronic processes and the new $\psi$ particles dominated recent research. Many studles dealt with various aspects of strong interaction dynamics, with $\psi$ particles and color, and with SU(4) or higher symetries, gauge 


\section{LAWRENCE BERKELEY LABORATORY \\ Berkeley, California}

theorles and weak interactions. In addition there was research on quantum field theory, on S-matrix theory and related aspects, together with many on miscellaneous topics.

New dispersion relations, derived on the basis of S-matrix theory, have been used to give a systematic development of Regge theory starting from basic analyticity properties.

Accelerator theorists have continued a broad range of projects on the design of accelerator systems such as PEP, ESCAR, and ISABELLE, as well as more fundamental studies of theoretical problems of beam dynamics.

Research on accelerators is done primarily in close collaboration with experimental physicists and engineers for the purpose of defining the parameters of a new facility or for understanding and improving the behavior of existing accelerators. A heavy-ion accelerator for cancer therapy was recently designed at LBL. 


\section{STANFORD LINEAR ACCELERATOR CENTER \\ Stanford, California}

Contract No.

EY-76-C-03-0515

Title of Project: Experimental Group A

Person in Charge: R. Taylor

Scientific and Professional Staff: W. Atwood, E. Bloom, R.

Cottre11, H. DeStaebler, M. Mestayer, C. Prescott, L. Rochester, T. Sato

\section{Scope of Work}

The group will continue studies of deep inelastic electron scattering to obtain information about nucleon structure and the electron-nucleon interaction. Our immediate experimental program includes the development of a high intensity source of polarized electrons to be used in a search for a parity nonconserving componént in the electron-nucleon interaction. Part of the group is engaged in the construction of a neutral particle detector for experiments at SPEAR, and part is also engaged in design of experimental equipment for PEP.

Goa1s and/or Major Results to Date

Using the large magnetic spectrometers, designed and constructed in large part by this group, we have carried out a series of experiments aimed at exploring the electron-nucleon interaction in the kinematic region available at SLAC. A most important and far-reaching result of the early experiments was the discovery that the neutron and proton appear to be composed of point-like constituents, nowadays called partons, and subsequent work has refined and extended the early measurements. In other experiments we searched, unsuccessfully, it turned out, for the violation of time-reversal invariance and for parity non-conservation in the electron-nucleon interaction.

$$
* * * * * * * * * * * * * *
$$

Title of Project: Experimental Group B (and LASS)

Fersoü in Chargc: D. Leith

Scientific and Professional Staff: W. Johnson, T. Lasinski, S. Shapiro, P. Kunz, W. Dunwoodie, W. Meyer, B. Ratcliff, D. Hutchinson, S. Williams, R. Champion, R. Bierce, .T. Fond, M. Gravina, H. Hanerfield, J. Persing, K. Stewart 
STANFORD LINEAR ACCELERATOR CENTER

St anford, California

$\underline{\text { Scope of Work }}$

The research interests of Experimental Group B are focused 1argely on experiments with on-line wire spark chamber instrumentation, and also on the development and installation of the Large Aperture Solenoid spectrometer (LASS). This work is being carried out in collaboration with physicists from Johns Hopkins University and from the California Institute of Technology. Group B is analyzing data on $\mathrm{Kp}$ elastic and inelastic scattering from SLAC Experiment $75 / 85$, which predates the LASS facility. The Group is also preparing for the first round of experimentation with LASS.

Goals and/or Major Results to Date

Group B has been able to observe the crossover in the elastic cross sections for particle and antiparticle, and to measure its dependence on the identity of the particle and on the scattering energy. These studies provide basic information on particle "sizes" and also some insight into nondiffractive exchange mechanisms.

The new IASS facility is now in its early operational phase, with". the first experimental work just beginning. The first reaction to be studied will be pi-p at an energy of about $16 \mathrm{GeV}$; lepton-pair production in this reaction will be studied. Data on the complex LASS facility will be accumulated in order to facilitate later outside use of LASS by other experimenters.

$$
* * \dot{*} * * * * * * * * * *
$$

Title of Project: Experimental Group C (and Mark II Detector)

Person in Charge: B. Richter

Scientific and Professional Staff: A. Boyarski, M. Briedenbach,

R. Larsen, V. Luth, H. Lynch, R. Schwitters, W. Tanenbaum, D.

Hitlin, M. Alam.

Scope of Work

The research activities of Experimental Group $C$ are concentrated on experiments with the SPEAR colliding-beam facility at SLAC. The Group is also responsible for the design and development of a new large magnetic detector for use at SPEAR. This device, the Mark II, wil1 replace the existing Mark I at SPEAR. 
STANFORD LINEAR ACC̉ËLERATOR CENTER

Stanford, California

Goals and/or Major Results to Date

In collaboration with Group $E$ at SLAC and with members of a group from the Lawrence Berkeley Laboratory, Group $\mathrm{C}$ has continued its studies of hadron production in electron-positron annihilation at SPEAR. The "psion" family of heavy particles has now increased to at least 8 and perhaps 9 separate states, whose properties are consistent with the charm hypothesis of hadronic structure. Recently the group has discovered two heavy meson states, with masses of about $1.87 \mathrm{GeV}$, that appear to be the first clearly identified examples of charmed particles. Much work remsins to be dune to unravel the very complex structure noted in hadron production in the transitional energy region from about 3.9 to $4.5 \mathrm{GeV}$ at SPEAR.

$$
* * * * * * * * * * * * * * * \text {. }
$$

Title of Project: Experimental Group D

Person in Charge: R.F. Mozley

Scientific and Professional Staff: K. Bunnel1, M. Duong-Van, E. Kogan, A. Odian, F. Villa, L. Wang

Scope of Work

The group is nearing completion of Experiments E72 and E104 which study the inelastic scattering of muons on hydrogen and deuterium using the SLAC streamer chamber. The last papers are now being written for publication. An experiment (E110) searching for charmed mesons and baryons (using the same experimental setup as E/2 and E104) has been completed and the results published.

Goals and/or Major Results to Date

The group has just completed a 200-hour run on single and dimuon production in hadron-proton collisions (Experiments E123A and E123B). Several thous and events have already been measured and the data analysis is proceeding. A total of 30 -thousand dimuon triggers and 42-thous and single muon triggers have been taken. Two hundred more hours of running remain in these experiments and a request for 200 additional hours is being made so that if approved we shall have three or more times the present data.

The group is also considering a proposal for a streamer chamber at PEP.

$$
* * * * * * * * * * * * * *
$$




\section{STANFORD LINEAR ACCELERATOR CENTER \\ St anford, California}

Title of Project: Experimental Group E

Person in Charge: M. L. Per 1

Scientific and Professional Staff: J. Dorfan, G. Feldman, G. Hanson, F. Heile, J. Jaros, D. Luke, J. Martin, T. Pun, M. Stoddard, K. Wit thaus

Scope of Work

Group $E$ pursues two fields of research. One field consists of the study of the physics of electron-positron annihilation at high energy. The group is interested in the new, hadron-like particles which are produced relatively copiously in electron-positron annihilations. Examples are: the $\Psi$ family of particles, and what appears to have recently been discovered--singly charmed mesons. The group is also interested in studying the general dynamics of hadrons as exhibited in the annihilation production of hadrons.

The second field of research, which the group has pursued since the formation of SLAC, concerns the fundamental nature of the charged leptons (the electron and the muon); and the search for heavier members of that lepton family.

Group E is also concluding a series of studies of the electroproduction of hadrons, a process which studies the internal structure of the hadrons.

Goals and/or Major Results to Date

The collaboration of Groups $C$ and $E$ at SIAC and the GoldhaberTrilling Group at LBL has produced many new results on new particles, as we 11 as ordinary hadrons, produced in electron-positron annihilation processes: Some examples are: the finding of the $\Psi$ family of particles and the discovery of jet structure among the hadrons produced in the annihilation process.

of particular importance has been the discovery of electron-muon events, the events which have been interpreted as the pair production and subsequent decay of the $U$ particle. The simplest explanation of the nature of the $U$ particle is that it is a heavy lepton.

$* * * * * * * * * * * * * *$

Title of Project: Experimental Group G (and DELCo Detector)

Person in Charge: M. Schwartz 
STANFORD LINEAR ACCELERATOR CENTER

Stanford, California

Scientific and Professional Staff: S. Wojcicki (Stanford), J. Kirkby, R. Piccioni, J. Liu, R. Coombes, D. Porat

Scope of Work

Experimental Group $G$ consists of physicists from SLAC and from the Stanford Physics Dept. The group's research interests are presently directed principally toward studies of direct lepton production both from electron-positron annihilation and from hadronic production.

Goals and/or Major Results to Date

During the past year Group $G$ has completed the analysis of an experiment in which $\mathrm{K}^{\mathrm{O}}$ mesons were incident on protons in a $15^{\prime \prime}$ rapidcycling bubble chamber at SLAC. In this work the $\mathrm{K}^{\circ}$ spectrometer facility was used to provide an effective trigger for the huhble chamber.

A major project of the group is the construction a "direct electron counter" (DELCO) for use in an experiment at the SPEAR storage ring. This device will provide large solid-angle coverage--about $65 \%$ of 2 pi--for electron detection and will have a hadron rejection of: about $10^{-4}$. The group has built the cylindrical wire chamber array for this device and has designed much of the needed electronics for the experiment.

$$
* * * * * * * * * * * * * *
$$

Title of Project: Experimental Group BC

Person in Charge: J. Ballam

Scientific and Professional Staff: R. Aintablian, R. Blumberg, J. Bouchez, T. Carro11, G. Chadwick, V. Chaloupka, C. Fie1d, D.

Freytag, R. Lewis, H. Petersen, R. Stevens

\section{Scope of Work}

This group is currently performing experiments using the 40-inch bubble chamber hybrid facility. It provides support for experiments done by uocro mitidi BLAC ds well ae proposing and execulling its own programs. The equipment of the facility includes a computer, electronic counters, proportional wire chambers and a large Cerenkov counter used in conjunction with the bubble chamber to allow the triggering of photographs only on selected classes of events. Addition and improvements to the equipment are planned and executed by the group as we 11 . 


\section{STANFORD LINEAR ACCELERATOR CENTER}

Stanford, California

Beams which ${ }_{ \pm}$an be used with the system are: $\pi^{ \pm}$mesons up to $18 \mathrm{GeV} / \mathrm{c}, \mathrm{K}^{ \pm}$mesons up to $11.6 \mathrm{GeV} / \mathrm{c}$, and antiproton beam up to $9 \mathrm{GeV} / \mathrm{c}$. Because of the geometry of the detectors, the facility is particularly useful to study diffraction and other peripheral exchange processes where a fast forward particle provides a signature. However, work is in progress to extend the triggering capability to more general reactions by recognition of event patterns within the chamber itself.

Goals and/or Major Results to Date

The group has performed experiments (generally in collaboration with other groups) on inelastic $\|$ meson-proton scattering (5000 events $/ \mu \mathrm{b})$, inelastic diffraction scattering of $14 \mathrm{GeV} / \mathrm{c} \pi^{\text {s }}$ on protons ( 170 events/ $/ \mathrm{b})$, and is currently studying the production mechanisms of excited hyperon states produced by $11.6 \mathrm{GeV} / \mathrm{c} \pi$ and $\mathrm{K}^{-}$protons (500 events/ $\mathrm{bb}$ each). It now plans to study events with "anomalous" direct production of electrons in $\pi$ meson-proton interactions to determine the source of such particles. It will provide assistance to outside groups approved to study $\pi^{\circ}$ production in $\pi p$ collisions (Duke University) and antiproton annihilation cross sections at 9. GeV/c (Michigan State University and Cambridge University).

$$
* * * * * * * * * * * * * * *
$$

Title of Project: Theoretical Physics

Person in Charge: S. Dre11

Scientific and Professional Staff: S. Berman, J. Bjorken, R.

Blankenbecler, S. Brodksy, S. Dre11, F. Gilman, H.P. Noyes, Y.S. Tsai, M. Weinstein, W. Caswe11, G. Chu, R. Giles, A. Hanson, H. Miettinen, S.H. Tye, H.C. Tze, A. Weldon, S. Yankielowicz.

\section{Scope of Work}

The work of SLAC's Theoretical Physics group continues its tradition of close contact with experimental discoveries and developments. In addition, the group carries out fundamental studies of the forces and particles involved in elementaryparticle research. Since the discovery of the psi particles in late 1974, there has been a strong emphasis on analyses and interpretation of these remarkable new particles. 


\section{STANFORD LINEAR ACCELERATOR CENTER \\ Stanford, Calif̣ornia}

Goals and/or Major Results to Date

Studies of strong-interaction dynamics and, in particular, of 1 arge transverse momentum dynamics have continued; general laws for the energy dependence of secondary multiplicities in electron-positron annihilation and in hadron-induced processes have been constructed using the theoretical ideas of quark-gluon dynamics.

Much effort has been devoted to the important problem of understanding. the new states of psionic matter. Phenomenological analyses of the new particles, their spectroscopies and decays have been carried out lu see whether they can be understood as bound states of only one new quark and its corresponding antiquark. There has alsn hepn speculative work on new models of the psi particles as well as analyses of the anomalous mu-e events reported from the SPEAR storage ring at SLAC.

A major effort to understand the dynamics of quark confinement in terms of strings and of lattice gauge theories is in progress. A theory of quark binding strings has been developed, and its application to the psionic spectra carried out. Studies of the geometric structure of strings and of the topological conservation laws associated with extended field structures are in progress, along with extended studies of strong-coupling gauge-field theories formulated on a spatial lattice.

$$
\text { 安 }
$$

Title of Project: Data Analysis

Person in Charge: J. Brown

Scientific and Professional Staff: L. L. Barker, D.C. Burwe11, V.I. Hamilton, A.J. Leino, P.A. Malley, R.A. Moyer

Scope of Work

The objective of the Data Analysis Group is to design, construct, operate and maintain scanning and measuring equipment for the analysis of film recorded data from streamer chamber and bubble chamber experiments. This includes not only manually uperaled machines but also a semiautomatic machine (the Spiral Reader). This latter device processes track-chamber film with high accuracy at rates significantly greater than is possible with conventional techniques. As a secondary objective, the group participates in the development and construction of hybrid or non-film data analysis equipment, insofar as such activity arises naturally from the efforts above. 


\section{Goals and/or Major Results to Date}

During the past five years, the following quantities of bubble and spark chamber film have been measured and scanned:

\section{Frames Scanned Events Measured}

$\begin{array}{lrr}\text { FY } 1972 & 2,000,000 & 812,000 \\ \text { FY } 1973 & 650,000 & 409,000 \\ \text { FY } 1974 & 690,000 & 264,000 \\ \text { FY } 1975 & 708,000 & 190,000 \\ \text { FY } 1976 & 628,000 & 125,000\end{array}$

$* * * * * * * * * * * * *$

Title of Project: Accelerator Operations

Person in Charge: V. Price

Scientific and Professional Staff: J. Jasberg, G. Gilbert, D. Tsang, J. Sirois, J. Truher, T. Inman, W. Asher, G. Nelson, L. Stein, R. Hanse1man, H. Joseph, R. Hage1

Scope of Work

The. Accelerator Operations Group is responsible for the operation and maintenance of the SLAC two-mile linear electron accelerator. Operation of the accelerator continues to be reliable, with beams available to the experimental area approximately $90 \%$ of the scheduled running time. When operating in the multiple-beam mode, with typically 5 to 6 experiments sharing beams, reliably still exceeds $75 \%$ of the scheduled running time. Electron beam energies up to a maximum of $22.7 \mathrm{GeV}$ are routinely achieved.

Goals and/or Major Results to Date

During FY 1976 the accelerator ran for 660 eight-hour shifts of experimental operation. A significant portion of this running time was a pulse repetition rates lower than the maximum of 360 pulses per second in order to maximize the operation of the SPEAR storage ring. For FY 1976, this running plan resulted in the delivery of about $35 \%$ of the maximum feasible number of beam puises throughout the year.

The forecast for FY 1977 is for a reduction in operating shifts to about 641 , with total pulse generation down to perhaps $31 \%$ of 


\section{STANFORD LINEAR ACCELERATOR CENTER \\ St anford, California}

maximum. It is hoped that the funding for subsequent years will be such as to permit an increase in this relatively low level of exploitation of the SLAC accelerator.

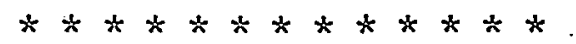

-Title of Project: Experimental Facilities Operations

Person in Charge: L. Keller

Scientific and Professional Staff: D. Fryberger, R. Gearhart, T. Fieguth, J. Murray, J. Harris, E. Johnson, C. Haxris, M. Berndt, C. Guracar, F. Veldhuizen, J. Lipari, H. Weidner, A. Baumgarten, F. Halbo, R.S. Larsen, E. Faust, E. Horelick, J. Mark, W. Pierce, H. Kang, K. Henderson, J. Hall, F. Halbo

\section{Scope of Work}

The Experimental Facilities Department (EFD) is responsible for the engineering support, installation support, and operation of facilities in the research area, including the SPEAR interaction areas. A11 experimental groups from the outside user community and from SLAC are supported by EFD. In addition, EFD provides assistance and coordination in the research area activities of other groups, such as the Spectrometer Facilities Group, the SPEAR Group, and the Hydrogen Bubble Chamber Group, which operate and maintain facilities in this araa.

EFD is responsible for design, erection, and maintenance of appropriate secondary beam lines in accordance with the approved and pending experimental schedule. In addition, EFD provides general purpose equipment, such as hydrogen targets, analyzing magnets, electronics, power supplies, etc., which are used in the setup of experiments in the research area. Electronic instrumentation service is in the form of direct electronics engineering and technician support for development, construction and installation of complete data acquisition and control modular experimental instrumentation systems through a high energy electronics pool (HEEP). A central design and development group works closely with HEEP and with interlaboratory standards groups in developing standard instrumentation approaches for experimental. syst.ems. Design work includes detector electronics, data acquisition and control systems, computer interfacing, and some software. 


\section{STANFORD LINEAR ACCELERATOR CENTER \\ St anford, California}

Goals and/or Major Resu1ts to Date

During FY 1976 some 16 experiments and a large number of detector tests were set up in the SLAC research yard. Facility construction during the past year has included the Large Aperture Solenoid Spectrometer (LASS), the SLAC Hybrid Bubble Chamber Facility (SHF), energy upgrading of the beam transport line to SPEAR, modification of the test beam transport line to handle low-energy particles, and expansion of the cable plant and control room space at the east interaction area of SPEAR. In order to increase the amount of crane-covered experimental assembly space available, the inactive $82-$ inch bubble chamber was dismantled and removed.

EFD personnel are also participating in the development of plans for research area changes that will be needed to accommodate the higher energy beams expected from the SLED project, and also in the design of the interaction-region experimental areas for the PEP storage ring.

Electronics instrumentation development work has focused on the requirements of two major new detectors now under construction: the Mark II magnetic detector and the Crystal Ball detector, both: to be used: with the SPEAR storage ring.

$$
\star * t * * * * * * * * * *
$$

Title of Project: Bubble Chamber Operations

Person in Charge: $R$. Watt

Scientific and Professional Staff: R. Rinta

Scope of Work.

The Bubble Chamber Operations Group is responsible for the operation and maintenance of the SLAC bubble chambers, the central helium facility, and the cryogenics part of the LASS superconducting magnet. This group is also participating in the design of the cryogenic support system. for the Mark II SPEAR detector.

Goals, and/or Major Results to Date

The 40" bubble chamber has operated in a routine fashion at pulse rates up to 12 pulses per second. The hybrid system that wil1 be used to provide a selective trigger to the $40^{\prime \prime}$ chamber is now in operation and running satisfactorily. The $15^{\prime}$ rapid-cycling bubble chamber has operated well at 40 pps but is not presently in research 
use owing to the lack of proposals for experiments with this facility. The IASS cryogenic facility is now operating reasonably routinely, with only minor difficultied encountered.

$$
* * * * * * * * * * * * * * *
$$

Title of Project: SPEAR Operations

Person in Charge: B. Richter, J.M. Paterson

Scientific and Professional Staff: M. Lee, R. McConne11, A. Chao, P. Morton, A. Sabersky, G. Fischer, H. Wälz, J.I. Pellegrin

Scope of Work

The SPEAR group is responsible for the operation, maintenance and improvement of the SPEAR electron-positron colliding-beam storage ring facility. at SLAC. The SPEAR group is also responsible, in collaboration with SLAC Experimental Group $C$, for the design and development of a second-generation magnetic detector for use at SPEAR; this new instrument, the Mark II, will replace the original Mark I detector at SPEAR sometime during 1977.

The SPEAR group also collaborates with the PEP group at SLAC on certain aspects of the research and development work for PEP. PEP is a new construction project at SLAC that will result in an $18 \mathrm{GeV}$ electron-positron colliding-beam facility by about 1980 .

\section{Goals and/or Major Results to Date}

In recent months a higher energy injection system for SPEAR has been completed, installed and brought into satisfactory operation. "Injection is now carried out at an energy of $2.25 \mathrm{GeV}$, rather than the previous $1.5 \mathrm{GeV}$, and this modification has resulted in much reduced filling times and an increase in the maximumbeam currents that can be stored in the ring.

SPEAR now operates at its design luminosity over most of its energy range of about 1.5 to $4.0 \mathrm{GeV}$ (each beam). Above $3.4 \mathrm{GeV}$ per beam, the luminosity remains approximately constant, rather than increasing as expected. This effect is calsed by a heam-surrent limitation that results from the heating of vacuum chamber components. The very short beam bunches and correspondingly large peak currents in the ring excite electromagnetic fields of very high frequency, and in certain cases the power dissipated in vacuum chamber components can produce a significant temperature rise. Such higher order mode heating has been studied extensively in collaboration with members 


\section{STANFORD LINEAR ACCELERATOR CENTER}

\section{Stanford, California}

of the PEP group. Heat-sensitive components have been identified and are now being redesigned.

$$
* * * * * * * * * * * * * * *
$$

Title of Project: Spectrometer Facilities Group

Person in Charge: C.K. Sinclair

Scientific and Professional staff: W.W. Ash, D.J. Sherden, D.H. Coward

\section{Scope of Work}

This group provides technical support and assistance to experimenters using the large magnetic spectrometers and related facilities in End Station $A$ at SLAC. Experimenters both from within SLAC and from outside institutions are supported. The group is responsible for the operation and maintenance of the End Station A facilities, and the control, monitoring, interfacing and computational facilities. in the End Station A counting house. The group also carries out a development program aimed at improving the performance and efficiency of the experimental equipment. All of the members of the professional-staff of the group are actively involved in various aspects of the experimental program.

\section{Goals and/or Major Result's to Date}

Since formation of this group in 1969, a total of 23 particle-physics experiments have been carried out with the End Station A spectrometer facilities. Many of these have required novel equipment, the preparation of new beams, and occasionally entirely new detection apparatus. This group has supervised all of this activity, including the development and operation of three major new beams. The group has also participated in the development of a large-volume highfield polarized proton target. In addition, a new high-intensitysource of polarized beam electrons is presently under development. The control system for the three large spectrometers has been completely revamped, with excellent results. A computer-1ink system between the counting house computers and the main SLAC Triplex facility is now nearing operation. Design work is now in progress for increasing the aperture of the spectrometers and for extending their operation to higher energies in anticipation of the SLED accelerator energy increase program that is now under way.

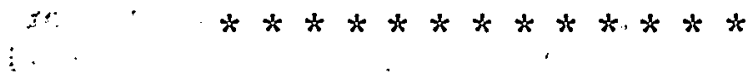


STANFORD LINEAR ACCELERATOR CENTER

Stanford, California

Title of Project: SLAC Energy Doubler (SLED) Project

Person in Charge: H. Hogg

Scientific and Professional Staff: Z.D. Farkas, G.A. Loew, C. Kruse

Scope of Work

A method of increasing the beam energy using transient radio frequency (RF) energy storage was invented at SLAC in 1973. A cavity with a provision of separating the powers flowing toward and away from the cavity io intcrposcd between the high puwer klystron and the accelerating section. For a short time after RF turn on, the bulk of klystron power is used to fill the cavity with RF energy. Reversing the phase of the RF, one filling time before the end of the pulse causes the power from the klystron and from the cavity (using stored energy) to combine and flow into the accelerator section. In this manner, with the present $2.5 \mathrm{ks}$ pulse, it is possible to increase the beam energy by $40 \%$. This is equivalent to adding 12 new sectors or 240 klystrons with their associated modulators. It is contemplated to increase the pulse width to $5 \mu \mathrm{s}$, which would make it possible to nearly double the beam energy. Hence the name SLED.

Goals and/or Major Results to Date

Presently, a program of converting the accelerator to operate in the SLED mode (compatible with normal operation) is being implemented. To date, August 20, 1976, two sectors have been converted and tested. The experimental results agree with the predicted beam energy gain. The average conversion rate is about a sector per month.

Theoretical research in conjunction with SLED were and are being carried out on the following topics:

1. Optimization of cavity parameters to obtain maximum energy gain for a given cávity volume.

2. Power dissipated in and temperature stabilization of the cavities.

3. Investigation of the response of high-Q cavities to pulses of microwave power as a function of frequency and phase.

4. Investigations of methods to optimize the energy spectrum and current of the beams to be obtained under a variety of conditions with SLED.

5. Investigations of methods to increase the beam breakup threshold of short electron beam pulses ( $<400 \mathrm{~ns}$ ) to be accelerated through the accelerator. 


\section{STANFORD LINEAR ACCELERATOR CENTER}

Stanford, California

6. Development of special instrumentation for assembly line testi $g$ of SLED cavities and for operating with a SLED beam.

The SLED concept uses a new method of pulse compression which uses $180^{\circ}$ biphase modulation and one or several resonators in tandem with a means of separating the powers traveling toward and away from each resonator, instead of linear frequency modulation and a dispersive structure. This new method has the potential for uses other than beam energy enhancement. Further research will be done to place this new concept on a firmer theoretical foundation.

$\star * * * * * * * * * * * *$

Title of Project: Klystron Tube Development

Person in Charge: J.V. Lebacqz

Scientific and Professional Staff: B.E. Boesenberg, B.L. Byers, H.R. Greenhi11, B.A. Hayward, T.W. Johnston, P. Lee, A.D. Roach, W.R. Roberts, L. Tice

\section{Scope of Work}

The Klystron Group at SLAC is responsible for the development of klystron high-power sources for use with the SLAC two-mile accelerator and for use with the existing SPEAR and now in construction PEP electron-positron-storage rings.

Goals and/or Major Results to Date

\section{A. S-Band Klystrons for the Accelerator}

The drift tube lengths and cavity frequencies have been optimized in the standard S-Band klystron such that higher efficiency and output power $c$ an be achieved. Several tubes with minor variations have been built to demonstrate the validity of this approach. A11 of them yielded an output power between 36 and $40 \mathrm{MW}$ at $270 \mathrm{kV}$. The efficiency was between $46 \%$ and $49 \%$. This was accomplished by increasing the tube length by 1 to $3 \mathrm{~cm}$ compared with the standard $30 \mathrm{MW}$ tubes. This increase can be accommodated in the present permanent magnets. After a final design has been accepted, several pilot line tubes will be built and tested. It is expected that this tube will then become the standard gallery klystron. 
STANFORD LINEAR ACCELERATOR CENTER

Stanford, California

\section{B. UHF Klystrons for Storage Rings}

Five UHF klystrons operating at $358.5 \mathrm{MHz}$ and $125 \mathrm{~kW} \mathrm{CW}$ have been built. Four of these have been operating in SPEAR for almost two years. Computer studies indicate that a $500 \mathrm{~kW} \mathrm{CW}$ klystron with an efficiency of $70 \%$ can be built for PEP. The first tube, which was 1 Imited in average power by its collector, operated at $63 \%$ efficlency and put out $500 \mathrm{~kW}$ at $4 \%$ duty. It has also been operated up to $200 \mathrm{~kW} \mathrm{CW}$ at reduced voltage into a dummy load and a 2 -cell PEP cavity. Another tube with full $500 \mathrm{~kW}$ CW capability will go into test in late summer of 1976.

***************

Title of Project: Positron Source

Person In Charge: G.A. Loew; C. Kruse

Scientific and Professional Staff: R. Miller, A. Lisin

Scope of Work

In recent years the primary use of the positron source is to produce a positron beam to infect into the SLAC Positron-Electron starage ring, SPEAR, Since SPEAR stores only a single R.F. bucket of positrons, a very short pulse $(\sim 1.5 \mathrm{nsec})$ of positrons of very high incensity is required. SPEAR fills at a maximum rate of 60 pps and a maximum energy of $2.25 \mathrm{GeV}$ pps while the accelerator runs normally at elther 180 pps or 360 pps and most other beams are about $20 \mathrm{GeV}$. Thus the SPEAR hIgh intensity ohort pulse low energy positron beam must be interlaced on a pulse to pulse basis with long pulse, high energy electron beams. During the three years SPEAR has been operating, the stability, average intensity, and compatibility with standard electron beams has been improved tremendously.

Peak positron beam intensities as high as $10 \mathrm{~mA}$ have been achieved, but are by no means routine. The PEP $15 \mathrm{GeV}$ positron electron beam which will be completed in 1980, requires a very similar, though higher energy beam. While the present positron intensities will be adequate for PEP, becauoc of tits higher stured charge, higher intensitfes will be required for good operation (1.e. good ratio of storage time to fill time): A number of possible improvements are being studied for increasing the high peak current; low average power performance of the positron source. These include: higher density targets, improved magnetic focusing, and a modified microwave accelerator section which would start accelerating the beam 
STANFORD LINEAR ACCELERATOR CENTER

Stanford, California

closer to the target. Any changes made must permit operation of the positron source with high average power for experiments scattering positrons off fixed targets.

Goals and/or Major Results to Date

We hope to achieve routine operation with peak currents for short pulses ( $\sim 1 \mathrm{nsec}$ ) between 10 and $20 \mathrm{~mA}$ within the next few years, and long pulse ( $1.6 \mathrm{Hsec}$ ) high repetition rate (up to $180 \mathrm{pps}$ ) currents of 2 to $4 \mathrm{~mA}$.

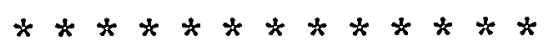

Title of Project: Polarized Electron Source

Person in Charge: G.A. Loew, C. Kruse

Scientific and Professional Staff: R. Mi1ler, J. Sodja (In collaboration with V. Hughes, $M$. Lubell and J. Clendenin of Yale University; and G. Baum and $W$. Raith of the University of Bielefeld, West Germany.)

Scope of Work

Polarized Electron Source from Lithium Atomic Beam:

The polarized electron source has been operational on the accelerator since November 1974. Developed by a Physics group headed by Vernon Hughes at Yale University in collaboration with SLAC, the source was installed starting in April 1974. Since its first operational use in the fall cycle of 1974 the intensity has been increased by an order

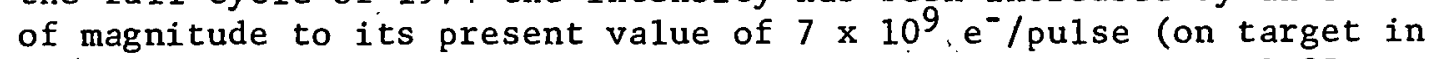
experimental area). The polarization at this intensity is 0.85 . At present a factor of higher intensity is available in a different operating mode with a polarization of $\sim 0.5$.

Goals and/or Major Results to Date

It is hoped that continued development will yield another factor of 3 in intensity at a polarization of 0.85 and increase the duty factor from its present value of about 0.6 to about 0.8 . This source with its high polarization but limited intensity is well suited for use with polarized proton targets. It will be used in a series of experiments testing the theories of nucleon structure.

$$
* * * * * * * * * * * * *
$$




\section{STANFORD LINEAR ACCELERATOR CENTER \\ Stanford, California}

Title of Project: Injector Development

Person in Charge: R. Miller, G.A. Loew, C. Kruse

Scientific and Professional Staff: R. Koontz, T. McKinney

Scope of Work

The injector of the SLAC accelerator is the subject of a continuous updating and improvement program designed to keep beam generation capablifies ahead of current experimental requirements. In the recent past, this has entailed research and devclopment in the fields of electron gun design, fast pulse amplifier design, transverse beam choppers, fast pulse isolation transformers (Patent 3614694), and fast beam synchronization systems. We have successfully designed systems for injecting one nanosecond pulses into the SPEAR rings, we have loaded up to $10^{9}$ electrons into just one accelerator R.F. bucket for time-of-flight experiments, and we are presently developing new systems to accommodate the PEP storage ring:

In gun design, an inexpensive commerically available cathode-grid as sembly has been designed into a new gun structure. The structure optics have been extensively computer analyzed. The resulting gun is ideally suited for very fast (1-2 nanosecond) electron beam pulse generation. In fast amplifier design, a computer program has been written which models the non-linear properties of saturated switching tube amplifier stages, and predicts both the stage-gain and pulse shape characteristics of the amplifier stage. This program has been useful in developing better high voltage fast pulse amplifiers.

\section{Goals and/or Major Results to Date}

We expect to continue work in the development of more versatile electron beam injector systems. This will involve continued research into new methods of very fast beam generation, fast beam monitors, and more versatile synchronization systems to match pulse electronics to the R.F. bucket timing of the accelerator. While we have already produced beams of adequate intensity and stability for PEP injection, we expect to develop additional higher intensity beam sources to make PEP operation more reliable. In addition, we expect to start work with microprocessor systems for use in updating and expanding portions of the injector control system.

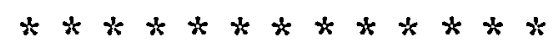




\section{STANFORD LINEAR ACCELERATOR CENTER \\ Stanford, California}

Title of Project: Beam Containment Project

Person in Charge: G.A. Loew, C. Kruse

Scientific and Professional Staff: K. Crook, T. Constant, D. Heggie

Scope of Work

The continual increase in beam power at SLAC, from an initial $200 \mathrm{~kW}$ to the present capability of $900 \mathrm{~kW}$, has caused a corresponding increase in the problems associated with absorption and containment of the beam. In order to prevent damage to mechanical devices and to detect the potentially hazardous beam conditions, an extensive elcctronic protection system was designed and installed. Various beam sensors are connected to electronic modules which measure instantaneous and average beam power, repetition rate and beam loss between selected points in a beam line. When an "out of tolerance" condition is detected, all beams are shut off immediately. If the abnormal beam condition is not detected, and a mechanical stopper dump or collimator is actually damaged, a burnthrough chamber called a "disaster monitor" located in or downstream of the device will rupture. This causes a pressure switch to turn off all beams and $\mathrm{RF}$ in the accelerator.

\section{Goals and/or Major Results to Date}

A11 presently required sensors, electronic processing and burnthrough monitors have been installed and are operating satisfactorily. Twenty channels of average power modules are connected to appropriate beam intensity monitors or secondary emission monitors (SEM's). Twelve comparator channels have been installed to measure instantaneous power at upstream locations and compare this with the power at measuring points downstream! If the difference exceeds a present value (typically 70\%) all beams are turned off. Six repetition $x$ ate monitors detect higher than normal beam rates. Six errant beam detectors. monitor the beam pulses and shut off all beams if an unprogrammed beam pulse reaches a beam line. In addition to the circuit described above, which operates on the $1.6 \mathrm{\mu s}$ beam pulse, there are approximately 50 DC interlocks such as magnet setting, flow switches and vacuum valves that are monitored for correct operation.

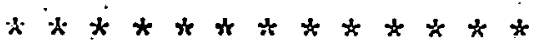

Title of Project: Accelerator Computer Control Improvements

Person in Charge: G.A. Loew, C. Kruse 


\section{STANFORD LINEAR ACCELERATOR CENTER \\ Stanford, California}

Scientific and Professional Staff: K.B. Mallory, S. Howry, W. Struven, R. Johnson

\section{Scope of Work}

The computer system used to control the accelerator and beam switchyard was overloaded before the first phase of work was completed. A study of the present and eventual needs was begun in 1971. The study was to determine how best to upgrade the computer control system, provide maximum flexibility, provide a system that is easy to operate and maintain, and to provide a plan whereby in 3-5 years the purchase and construction could be accomplished within anticipated budgets. The work was to cover investigations of the available computer systems and how they would fit in with the SDS925 and PDP-9 computers. Some of the system requirements were to provide for multiple control capabilities, increase the traffic capability of the two computer links, to increase reliability, to improve multiple beam operation, and to provide a means for checkout of programs while the accelerator was in operation.

\section{Goals and/or Major Results to Date}

A large portion of the program has been completed. The long term program has been accepted. About $50 \%$ of the program has been completed. A few of the major results are: PDP-8 computers have been installed in CCR and along the gallery; new computers have been purchased for increasing the capability of the MCC system, recorders for saving the latest programs in case of computer-memory failures, and additinnal computerc arc on order lo f1ll out the redundancy and reliability requirements in MCC. These new computers and associated equipment will provide partial computer (and touch pane1) control even though a failure occurs in the main computer. Additionally, the program is designed so that if required the SDS925 can be replaced with a new modern computer with minimum disruption and cost.

The relatively new devices available to aid in this program are microprocessors. This new concept has been utilized in various ways to further increase the capability and ease of operation of the system and to lecocn the loads on the central computers.

The completion of this long-range program is scheduled for FY 78 .

$$
* * * * * * * * * * * * * * *
$$




\section{STANFORD LINEAR ACCELERATOR CENTER \\ Stanford, California}

Title of Project: Energy Transient Compensation

Person in Charge: G.A. Loew, C. Kruse

Scientific and Professional Staff: H. Hogg, Z. Farkas, H. Martin, A. Wilmunder

\section{Scope of Work}

This project was to devise a means of eliminating an energy variation in the spectrum of the accelerated beam. Initial efforts used a. short pulse of a different phase than the normal length and phase to counteract an energy difference. The first system was workable but very unwieldy. Additional efforts under this program led to the concept of a computer or microprocessor-controlled digital phaseshifter. A study showed that this device if available could be . placed in the beginning of the accelerator and by proper spacing of a different but variable phase pulse in the normal RF pulse, would solve the problem. The project was basically to either purchase or build a digital phase-shifter and its attendant power supplies, driver and controller. A survey of. the commerical companies showed that indeed such a phase shifter was available. The program then was to obtain a suitable unit, test it, build up. the peripheral devices and install the total system in the accelerator.

\section{Goals and/or Major Results to Date}

Major results so far are: The pulsed phase shifter has been obtained and tested. It seems to perform as specified. The driver prototype has been built and tested. The remaining work is to design and. construct the microprocessor interface between the accelerator computer controls and the pulsed phase shifter driver. Installation and checkout on the accelerator would then follow.

$$
* * * * * * * * * * * * * * *
$$

Title of Project: Pulsed Focusing of High Current Beams

Person in Charge: G.A. Loew; C." Kruse

Scientific and Professional Staff: H. Hogg, R. Miller, L. Genova, K. Mallory 


\section{STANFORD LINEAR ACCELERATOR CENTER \\ Stanford, California}

\section{Scope of Work}

SLED operation requires that pulse width be exchanged for increased energy. Therefore, SLED is designed to utilize a shorter higher current pulse in order to extract the maximum energy from the RF power in the accelerator. This engineering requirement demands that the beam be very narrow $(0.3 \mu \mathrm{s})$ and of higher instantaneous current $(\sim 100 \mathrm{~mA} \mathrm{pk})$. This requirement means that stronger focusing is needed along the accelerator. To achieve this goal additional pulsed quadrupoles are proposed in key locations. Part of the impruvement is possible by increasing the number of quadrupoles in some areas and in other areas by increasing the strength of focusing. To this end studies have been made using computer programs to see if the transmission is adequate. The many iterations required have shown that one system or layout will give an acceptable result. In addition to more quadrupoles a means of supplying pulsed power in the order of twice what the present pulsed units require. Examination of the present quadrupole design was done to verify its capability to handle the increased current.

Goals and/or Major Results to Date

The major goal was to devise a system with stronger focusing using present designs and devices already installed for the lowest cost. First, the power supplies were designed and checked out to give higher currents. Second, the losses in cabling were reduced; third, the present pulsed quadrupole was tested at the new higher currents and found to be satisfactory. Fourth, ingenious relocation of present power supplies and controllers reduced the number of new items required. The new designs will be checked out in a special location on the accelerator during the next few months.

$$
* * * * * * * * * * * * * *
$$

Title of Project: Energy Spectrum \& Current Optimization Using SLED Beams

Person in Charge: G.A. Loew, C. Kruse

Scientific and Professional Staff: H. Hogg; Z. Farkus, H. Deruyter Scope of Work

R. Miller

The program is directed towards developing and examining several methods for optimizing the energy spectrum and current for various beams using the new SLED configurations. It is known that by use. 
of the beam loading characteristics of the accelerator we can nearly cancel the effect of the decay of power when the cavities are unloaded into the accelerator sections. The studies will examine the effects analytically and then correlate the results with actual beam tests. Other factors that can affect the energy spectrum and current are timing of the phase reversal, timing of the RF along the accelerator, tuning and tuning stability of the SLED cavities, and RF pulse shape. Many of these factors do not lend themselves to strict mathematical analysis so experimental methods will be used to obtain the overall net results.

Goals and/or Major Results to Date

The major goal in this study is to devise an easy system for operation of SLED so that.optimum operation becomes routine and high levels of skill and understanding are not required. To date most of the effort has been analytical with several actual beam tests proving that the system does work and $c$ an provide the anticipated energy gain. Two sectors with SLED cavities have been tested with a beam. Actual energy spectrum has only been inferred because the available spectrum monitors were incapable of displaying the spectrum across the total pulse width. This investigation will continue for approximately 2 more years. Better monitors and instrumentation will be available for the next series of tests.

$$
* \star * * * * * * * * * * * *
$$

Title of Project: Magnetic Harmonic Analysis Measuring System

Person in Charge: G.A. Loew, C. Kruse

Scientific and Professional Staff: J. Cobb, D. Jensen, J. Steffani, D. Horelick

\section{Scope of Work}

The program was to develop and test a multipole magnet measuring system. The proposed system would use a rotating long coil and mini-computer-controller. The computer would control the test and perform the fourier analysis calculations. Specifically it would sample the voltage output of the long coil (longer than the magnet) at 256 discrete points during each revolution. If the coil can be rotated at a constant velocity and the sample points measured accurately and at known spatial intervals, a discrete fourier transform $c$ an be used to determine the harmonic coefficients. These coefficients can be used to form an analytical expression 


\section{STANFORD LINEAR ACCELERATOR CENTER \\ Stanford, California}

of the magnet's characteristics which then can be used with beam transport programs. The advantage of this system is accuracy and speed of measurement.

\section{Goals and/or Major Results to Date}

This program is essentially complete. Magnets have been measured using the complete system. Accuracy and speed over other methods are better than expected. The first major user of this advanced system will be the PEP group. PEP has many multipole magnets to be designed and measured. The system has heen and will be in the fulure extremely valuable in the design phase of PEP and indispensible in the production testing phase.

$$
* * * * * * * * * * * * *
$$

'Title of Project: Microprocessor-Based Automatic Phasing System

Person in Charge: G.A. Loew, C. Kruse

Scientific and Professional Staff: H. Hogg, A. Wilmunder, S. Howry, K. Mallory

\section{Scope of Work}

The present phasing system was designed 12 yeare ago using llie analog Lransistor technology available at the time. Although it has been generally successful it is no longer possible to modify it to fit the present and projected requirements for new systems such as SLED and PEP. Additionally the requirement for a 60 pps beam with full use of that beam during phasing consumes more beam time than is necessary using newer digital techniques.

While studying system redesign the following possibilities were considered: eliminating the dependence on 60 pps pulse rate and allowing phasing at rates as low as 1 pulse in 10 seconds, pernitting the continuous phasing of the accelerator by stealing a pulse periodically, this would constantly give the best spectrum possible and eliminate down time due to having to rephase; allowing the independent phasing of a single klystron by means of a simple command; reducing the number of beam pulses needed to phase, improving the overall phasing accuracy, especially in Sectors 1 and 2; eliminating high failure rate components such as electrolytic capacitors; improving the general temperature characteristics of the system especially reducing the drift of sample gates; making the sample gate time delay variable to permit phasing during SLED mode; and, designing in sufficient flexibility to allow future changes with minimum hardware modification. 


\section{STANFORD LINEAR ACCELERATOR CENTER \\ Stanford, California}

Several different approaches were studied using both analog and digital techniques but the one using an 8080 microprocessor was judged to be superior to the others. Implimentation of this system requires replacement of the Programmer and Electronics package, but the RF systems of the RF Detector Panel and phase shifters would be used after minor modifications. It will also be necessary to change some of the cross connects in the alcove but no new cables will be needed.

The programmer will be converted from relay logic to microprocessor control. The electronic chassis will be converted to "NIM BIN" technology and digital circuits and techniques will be used throughout. A key change will be in the drive motor in the isolatorphase shifter-attenuator unit. The present servo-type motor will be replaced by a stepper type motor which lends itself to digital control. Other benefits attendant with this motor is constant torque for any error, no overshoot, inexpensive, longer life, and are programmable by applying a given number of drive pulses.

Goals and/or Major Results to Date

A complete system has been constructed which represents a sector in every way except for having only two phase shifters, one calibrating and klystron phase shifter. The remaining phase shifters only have their drive motors connected. A pulsed RF signal source puts out pulse pairs representative of the usual accelerate and standby pulses on the accelerator and these pulses can be individually switched on or off. A number of different programs have been tried with good success.

The first program tried took advantage of the stepping motor's characteristic of requiring a given pulse pattern to rotate a specific number of degrees. Motors were then programmed to rotate a specific number of degrees and stop. This same program was then expanded to drive all motors simultaneously so that a given phase shift could be programmed into the entire sector to replace the sub-booster phase shifter if desired. Another program has allowed the system to phase a klystron then throw an error into the system and then rephase the klystron. Observation. of this program shows that the system easily can maintain phase within 1/2 degree with a total of 10 beam pulses required. $R F$ pulses are not required during motor rotation.

System software problems are still being worked on and a prototype system is expected to be ready for installation and test during the next few months.

$$
* * * * * * * * * * * * *
$$


STANFORD LINEAR ACCELERATOR CENTER

Stanford, California

Title of Project: High Power Beam Intercept Studies and Instrumentation

Person in Charge: D. Walz

Scientific and Professional Staff: W. Brunk, B. Sukiennicki

Scope of Work

The scope of research and development activities of this group are to develop and test specialized beam handing apparatus for highenergy, high-power electron accelerators. Specifically, these devices are momentum-defining slits and collimators, beam dumps and stoppers, solid targels for production of secondary particle beams; also pulsed and dc electromagnets; also beam sensing instrumentation such as intensity, intensity profile, positron and profile monitors as well as spectrum analysers. Some of the groups efforts are devoted to the development of personnel protection and beam containment programs and associated hardware; also to the testing and evaluation of materials in a high-radiation environment. A small effort is devoted to problems associated with the safe operation of radioactive water systems, such as the safe disposal of radiolyticallyproduced gases and related radio-chemistry problems. The group participates in programs on isotope research for health physics and medical applications. Finally, a sma11, continuous effort is devoted to the search for superheavy elements, in targets irradiated in the accelerator as well as in nature.

Goals and/or Major Results to Date

Recent accomplishments are the development of compact high-power, medium-Z slits, collimators and dumps utilizing a bed of watercooled spheres, the development of a $\mathrm{H}_{2}-\mathrm{C}_{2}$ recombiner for radioactive water systems of particle accelerators; also the development of a beam containment system utilizing the data generated in destructive irradiation of materials with the high-power SLAC $\mathrm{e}^{-}$beam; also the development of a very efficient pulsed bending magnet utilizing tape-wound cores; further the development of a fast-response highsensitivity current monitor for storage ring injection systems, the development of a rabbit facility for isotope research (parasitically).

The search for superheavy elements has recently yielded strong evidence for such an element (spontaneously-fissioning with long half-life) or chain of elements existing in nature; also there is some evidence for at least one such element produced by the SLAC electron beam in a high-z target. 


\section{STANFORD LINEAR ACCELERATOR CENTER \\ Stanford, California}

Studies will continue on some of the above programs, also on the development of a high-power, high-z liquid metal target and on better magnet insulations.

$$
* * * * * * * * * * * * * *
$$

Title of Project: 40-Inch Hybrid Bubble Chamber Facility

Person in Charge: R.C. Field

Scientific and Professional Staff:. D.R. Freytag, R.A. Lewis

Scope of Work

The design, construction, and initial operation of the Hybrid Facility have been accomplished. Detectors provided for definition of the beam particles include multiwire proportional chambers, ; scintillation counters and a Cerenkov counter. To examine interaction products leaving the bubble chamber, a set of proportional chambers have been developed for the magnet exit aperture. These chambers are of a special design with a very small inactive area. A pressurized Cerenkov counter with large aperture and wide momentum ranges for particle identification has been constructed, backed by a scintillation counter hodoscope. The electronics for these detectors allows the acceptance of two independent events per accelerator pulse. The positions of the trajectories and Cerenkov pulseheights are digitized and transmitted to the Facility's NOVA 840 computer via a CAMAC interface. The computer $c$ an then make the final decision on taking a picture of the tracks in the bubble chamber.

Modifications, improvements, and extensions of the system for a variety of proposed experiments are presently in hand. Beams of $\mathrm{K}^{ \pm}$mesons and $\overline{\mathrm{p}}$ are anticipated in future running and optimization of these is in progress.

\section{Goals and/or Major Results to Date}

The Hybrid Facility has collected about $30 \%$ of the data for three experiments interested in the hypercharge exchange reactions $\pi^{+} \mathrm{p} \rightarrow \mathrm{K}^{+} \Sigma(1385)^{+}$, strange meson production, and forward $(\overline{\mathrm{p}})$ production. Preliminary analysis indicates that the system is operating as expected with no serious biases.

Within the next year it is intended to complete this data taking, perform a search for direct lepton production at a sensitivity of $\mathrm{e} / \pi \lesssim 10^{-4}$, and carry out an exposure analogous to the present one 


\section{STANFORD LINEAR ACCELERATTOR CENTER \\ Stanford, California}

but with a $\mathrm{K}^{-}$beam. Beyond this there is an experiment approved to study aspects of $\bar{p}$ p interactions.

$$
* * * * * * * * * * * * * * *
$$

Title of Project: Crystal Ball Project

Person in Charge: R.E. Taylor, E.D. Bloom

Scientific and Professional Staff: F. Bulos, G. Godfrey, C. Kiesling

Scope of Work

The Crysta1 Bal1 (CB) is a NaI(T1) detector of 677 individually phototubed segments. This device coordinated with charged parlicle tracking chambers, endcap detectors, and a precision luminosity monitor will be used at SPEAR II. The C.B. is designed to probe the neutral energy component at SPEAR II, assuming most of this neutral energy is ultimately gamma rays, measured to be over $50 \%$ of the total energy at $\mathrm{E}_{\mathrm{CM}}=7.8 \mathrm{GeV}$. The C.B. is capable of energy resolution of $Y^{\prime} s \mathrm{Cm}^{\circ}$

$$
\frac{\Delta E_{Y}}{E_{Y}}=0.04 \text { (FWHM), } 50 \mathrm{MeV}<\mathrm{E}_{Y} \approx 1000 \mathrm{MeV} \text {, }
$$

and angular resolution for $\gamma^{\prime} s$ of, $\Delta \theta_{Y} \lesssim 2^{\circ}$. Also, at SPEAR II the charmonium $\gamma$ ray spectroscopy presents a unique opportunity for a device with the characteristics of the C.B., and our collaboration intends to place much effort into obtaining information about this complex spectroscopy.

The large solid angle of the C.B. combined with our excellent $Y$ energy resolution, good $Y$ angular resolution, plus the charged tracking chambers will allow a complete final state reconstruction of a fair fraction of the events in the SPEAR II energy range. We will use these events to search for the presence of charmed particles. Also inclusive $\pi^{\circ}$, and $\eta^{\circ}$ distribution will be obtained.

The C.B., being a scintillation detector, also measures part of the energy of the charged particles in the final state. At SPEAR II the total final state energy measurement is sufficiently precise to allow a total energy trigger to be implemented. We believe this relatively bias-free trigger, combined with the large solid angle acceptance of the C.B. will allow a total cross section measurement of $\sigma_{\mathrm{e}}+_{\mathrm{e}^{-}} \rightarrow$ hadrons on the few percent level. 


\section{STANFORD LINEAR ACCELERATOR CENTER \\ Stanford, California}

Goals and/or Major Results to Date

The Crystal Ball Project is presently in a building phase. The project has just received ERDA approval of our CEP.

$* * * * * * * * * * * * *$ 
THIS PAGE

\section{WAS INTENTIONALLY LEFT BLANK}


PART II

FINANCIAL PLAN PROGRAMS 
AMES LABORATORY

Ames, Iowa

Contract No. W-7405-ENG-82

Title of Project: High Energy Physics - Theoretical

Person in Charge: W. Kernan

Scientific and Professional Staff: C. Hammer, K. Lassila, N. Dean, R. Leacock, B-L. Young, and L. B. Pilachowski

Scope of Work.

The general objective of Ames Laboratory investigations in theoretical high energy physics is the consfruction and application of theories and models of the strong, electromagnetic, and weak interactions that occur in high energy collisions of the elementary particles, e.g. In experiments at the Brookhaven National Laboratory AGS, at Ferm1 National Accelerator Laboratory, at the Argonne National Laboratory ZGS, and at Stanford Linear Accelerator Center. The theoretical effort can be divided into 1) formal theoretical studies, 2) phenomenological investigations, and 3) cooperative work with local and other high energy experimental groups. At the theoretical end of the spectrum, work is proceeding on spontaneous symetry breaking, condensate states in field theories, Klauder phenomena, high energy and large transverse momentum behavior of supersymetric field theories, and the fundamental physical basis for the role of complex angular momentum in strong interaction physics. On the phenomenological side there has been a study of two-particle to two-particle scattering data with Regge theory, a study of inclusive distributions with Mueller-Regge theory, and an analysis of charge and magnetic formfactor data. Recent interactions with the Ames Laboratory high energy experimental group involve the use of Reggelzed-Deck and multi Regge calculations to estimate cross sections and particle distributions for use in analyzing the feasibility of proposed experiments at the Stanford Linear Accelerator Center.

Goals and/or Major Results to Date

Theoretical and phenomenological effurls ili high encrgy phyeice are important as no theory for lifh energy processes exists; we share the national and international view that continuing understanding, development, and application of physical models will result in essential clues to the actual theory of high energy phenomena. Our goal is to make significant contributions to this development. We believe that our recent work on pion form factors, complex angular momentum trajectories and the work on supersymmetric field theories are important contributions.

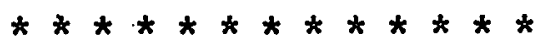


AMES LABORATORY

Ames, Iowa

Title of Project: High Energy Physics - Experimental

Person in Charge: W. Kernan

Scientific and Professional Staff: W. Kernan, E. Anderson, H. Crawley, A. Firestone, D. Parker, W. Higby, A. Klein, C. Fullhart

Scope of Work

This experimental program is directed towards investigating strong interaction processes at high energies. At the present time the effort divides naturally into analysis of data already taken at Fermilab and planning for a major experiment at SLAC.

1) The data taken at Fermilab was part of a four year continuing program. In this we studied $\pi^{-}$p interactions at 100 and $360 \mathrm{GeV} / \mathrm{c}$, pp interactions at 200 and $300 \mathrm{GeV} / \mathrm{c}$, and pd interactions at $300 \mathrm{GeV} / \mathrm{c}$. This program has concentrated upon measurement of information avai1able in the "high probability reactions" and the use of this information to understand hadron interactions at high energies. All experimental running has been completed, but the analysis work and publication is continuing.

2) Intensive work has begun in the last six months on plans for two experiments to utilize the LASS (Large Aperture Solenoid Spectrometer) facility at SLAC, The first experiment proposed will probably concentrate on $\pi^{+}$deuteron interactions yielding two or more charged $K$ mesons in the final state. Such events can be used in some test of theoretical models (the Zweig rule), to measure cross sections and angular distributions in reactions involving $\phi^{\circ}$ mesons in the final state, and as a measure of the reaction $\pi \pi \rightarrow K \mathbb{R}$.

Goals and/or Major Results to Date

The Fermilab experiments have been very successful in meeting their goal of studying hadron interactions at high energies. So far we have measured the total, elastic and topological cross-sections of $360 \mathrm{GeV} / \mathrm{c} \pi \mathrm{p}$ interactions, and tested "scaling invariance" to energies twice as high as previously reported. A theoretical model involving "local compensation" of transverse momentum has been developed and tested against the data. An interesting test of a pfediction of quantum electrodynamics has been carried out (direct $e^{-} e^{-}$pair production by a charged particle in hydrogen) and the data and theory agree. Our data also is the first experimental evidence that charged particle multiplicities do not rise like a power of the energy. 


\section{AMES LABORATORY}

Ames, Iowa

Our goal in the LASS experiment is to complete all design by October 1976 and to carry out the run during 1977.

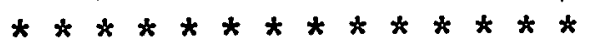


UNIVERSITY OF CALIFORNIA

San Diego, California

Contract No. EY-76-C-03-0010, 10-A.

Title of Project: Analysis of High Energy Interactions Using Nuclear Levels

Person in Charge: Oreste Piccioni

Scientific and Professional Staff: P. Bowles, B. Garland, W. Meh thop, D. Scipione, O. Piccioni, B. Barefield, J. McFarland

Scope of Work

The essential tool of analysis of high energy interactions is the study of the behavior of a particular interaction at various energies at different momentum exchanges. We are pioneering a new tool for such analysis, namely the study of the various interactions under the point of view of the nuclear levels which they excite, how probably they excite those levels, and, whenever such degree of freedom is available, how a level excited by high energy interaction deexcites itself. An example of the last property is the study of the direction of the photon emitted by the first level of Carbon when that level, after being excited by the interaction under study, returns to the ground state. An important point is that for levels which deexcite with photon emission, the detection of that photon can be made the first condition before any other apparatus is turned on; for instance, before spark chambers are triggered. Thus, despite the low probability that a nucleus remains together and at an excited level, this study can be carried on without too much of a waste of effort. Many levels are available, with known properties, such as parity, angular momentum and isotopic spin, and such properties are in general different for different levels, so that this study offers a multidimensional new possibility of analysis of elementary interactions, beside the classical analysis based on angular distribution and energy dependence.

One shortcoming of the method is that it cannot be used for large momentum exchanges to the nucleus, because the nucleus breaks apart if it recoils with a large momentum.

Goals and/ur Major Results to Date

The structure of a nucleus being a very complex one, it is expected that the probability of excitation of some levels might be a sophisticated probe. For that purpose it will not be necessary for a detailed understanding of the structure of a level. It will suffice, for example, to show that two types of resonances which 


\section{UNIVERSITY OF CALIFORNIA \\ San Diego, California}

are supposedly characterized by identical properties have instead different probabilities to excite a certain level. A rich network of relations and correlations will then be available to us, with which the tenets of the theories could be tested.

So far our work has mainly consisted in developing the method and in ascertaining its feasibility. However, data on the excitation of the first level of Carbon by pions and protons up to $5 \mathrm{BeV} / \mathrm{c}$ have been obtained, bearing on the diffractive nature of the elastic scattering of these particles. For contrast, the energy dependence of the excitation of the $15.1 \mathrm{MeV}$ level of Carbon, dramatically differs from that of the first level, showing that the inceraction responsible for the excitation of the $15.1 \mathrm{MeV}$ level decreases extremely fast with energy. This novel result has not yet been theoretically analyzed.

$$
* * * * * * * * * * * * * * *
$$




\section{CALIFORNIA INSTITUTE OF TECHNOLOGY \\ Pasadena, California}

Contract No. EY-76-C-03-0068

Title of Project: Neutrino Group

Person in Charge: B. Barish, F. Sciulli

Scientific and Professional Staff: A. Bodek, L. Stutte, M. Shaevitz, P. Mine, P. Linsay

Scope of Work

The Neutrino Program at Caltech is a joint Caltech-Fermilab project. A large detector was constructed at Fermilab in 1971 and a series of experiments were carried out until the fall of 1975 when the apparatus was dismantled.

The experiment showed that the general qualitative features of neutrino interactions at high energies are in striking agreement with the simple quark-parton picture. However, more recent results indicate some differences from this picture and it remains for future research to pursue and understand these effects.

The apparatus was also used for a variety of particle searches and mass limits have been set for $W$-bosons and heavy leptons. Although both searches gave negative results, apparent new. particle production has been observed in the experiment in $v$-events having two observed muons in the final state. Again, the source of these interesting events is not yet clear and remains for future experiments to determine.

The existence of neutral currents (neutrino interactions giving neutrinos instead of charged leptons in the final state) was convincingly demonstrated using the Caltech-Fermilab detector. More recent neutral current studies by our group have concentrated on determining the nature of the coupling. In the past year it has been sliuwn that the coupling is consistent with Vector-Axial Vector coupling, and agrees with predictions of the Weinberg-Salam model both for the $v$, A combination and the strength of the neutral current coupling.

A final run on the original detector was conducted in the fall 1975 . This experiment concentrated on measuring normalized cross sections and a total of 20,000 events were collected. Inftial results on events with $2 \mu$ 's in the final state and tests of charge symmetry have recently been presented. Further analysis is proceeding on these data. 


\section{CALIFORNIA INSTITUTE OF TECHNOLOGY \\ Pasadena, California}

Goals and/or Major Results to Date

The Caltech group is now embarked on a major building program in the newly constructed Lab-E at Fermilab. A new large sophisticated Caltech-Fermilab $v$-detector is being constructed and will begin initial operation this fall. Also, a second experiment (Caltech-Stanford collaboration) to search for weakly decaying particles produced in hadron collisions will be underway shortly.

$$
* * * * * * * * * * * * * *
$$

Title of Project: Multiparticle Spectrometer Studies of Hadron Physics

Person in Charge: G. Fox, R. Gomez, I. Pine

Scientific and Professional Staff: C. Bromberg, G. Fox, R. Gomez, J. Pine, J. Scheid

Scope of Work

Three experiments will be done by the above group, all part of the initial programs at two multiparticle spectrometers we have helped construct:

1) Dileption Production (at SLAC, using the LASSS spectromerer).

2) High transverse momentum interactions at $200 \mathrm{GeV} / \mathrm{c}$. (At Fermilab, using the Fermilab MPS.)

3) Peripheral multiparticle physics at $50-200 \mathrm{GeV} / \mathrm{c}$. (At Fermilab, using the Fermilab MPS.)

The first experiment utilizes a multicellular gas Cerenkov counter and an array of shower counters to trigger on electron pairs produced, for example, by $16 \mathrm{GeV} / \mathrm{c}$ pions incident on protons. The anomolous production of leptons in hadronic reactions is currently of great interest and several theories purport to explain this phenomenon. Frnm the point of view of quark phenomenology the possible production of lepton patrs by quark-antiquark annihilalion is particularly interesting and can be studied in this experiment.

The second experiment is now in progress and consists of measuring the global properties of hadronic interactions in which a single high transverse momentum particle or a jet of such particlcs is produced. Calorimeters are used to trigger the multiparticle spectrometer on 


\section{CALIFORNIA INSTITUTE OF TECHNOLOGY}

\section{Pasadena, California}

such events. It is felt by many, and there is some experimental evidence in support of this, that such interactions result from hard collisions of hadron constituents (partons, or quarks).

The third experiment has at present only very general goals, which will be refined during the months before it begins. The spectrometer opens up many opportunities which must be evaluated in deciding which peripheral reactions are the most interesting to measure at this time.

\section{Goals and/or Major Results to Date}

Both spectrometer systems have successfully begun working during the past year: At SLAC, test runs on the dilepton experiment are presently under way. At Fermilab, data have been collected on the second experiment listed above, and more are now being obtained. The preliminary analysis of data indicates that the spectrometer can successfully be used to study the physics of reactions in which an average of ten charged particles are seen traversing the apparatus (up to 20 quite often in individual events).

Initial analysis of the high transverse momentum data shows evidence for jet structure and for relatively coplous production of high transverse momentum jets (In comparison.with single particles). In addition, the study of particles other than those triggering the apparatus shows interesting correlations which can help establish which theoretical models can in fact explain these high transverse momentum phenomena.

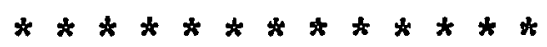

Title of Project: $\pi^{\circ}$ and $n$ Production Experiments

Person in Charge: A. Tollestrup, R. Walker

Sclentific and Professional Staff: A. Barnes, R. Kennett, D. Mellema, A. Tollestrup, R. Walker

Scope of Work

This group, in collaboration with groups from LBL and BNL, is engaged in three experiments at Fermilab studying $\pi^{\circ}$, and $n n$ (and other mesons decaying into photons) produced in $\pi \mathrm{p}$ and $\mathrm{pp}$ collisions. A new and very powerful hodoscope shower detector was developed for measuring the positron, energy, and mass of the decaying particles. 
Analysis of data obtained in our first experiment on pion charge exchange scattering, $\pi^{-} p \rightarrow \pi^{\circ} n$, and on the reaction $\pi^{-} p \rightarrow n n$, has been completed during the past year and the results are published in the Physical Review Letters. We believe these results provide the most significant high energy data available for these two reactions, which play a unique role in the study of Regge theory.

Our second experiment was a measurement of $\pi^{\circ}$ and $\eta$ inclusive production at high transverse momenta $\left(\mathrm{p}_{\mathrm{T}}\right)$, with emphasis on a comparison of incoming pion and proton beamo. It is belleved that particle production at high $\mathrm{p}_{\mathrm{T}}$ results from interactions of the constituents of the colliding particles. Therefore, it is important to compare reactions produced by different incident particles having different internal structures.

Our third experiment was begun in June 1976. It is a measurement of inclusive $\pi^{\circ}$ and $n$ production via reactions $\pi^{-} p \rightarrow \pi^{\circ} X$ and $\pi^{-} p+n X$ in the "triple Regge region." These reactions are the simplest ones to interpret in terms of tripple Regge theory so the results should provide a critical test of the theory.

Goals and/or Major Results to Date

A prediction for the difference in $\pi^{-} p$ and $\pi^{+} p$ total cross sections, $\Delta \sigma=\sigma_{\text {tot }}(\pi \mathrm{p})-\sigma_{\operatorname{tot}}\left(\pi^{+} \mathrm{p}\right)$, nhtained from our forwád charge exchange cross sections, agrees very well with recent direct measurements of this difference by Carroll et. al., at Fermilab.

Our experiment has provided the first data on particle production at high $\mathrm{P}_{\mathrm{T}}$ from IP collisions. We find that the ratio of cross sections $(\mathrm{pp} \rightarrow \pi \mathrm{T}) /(\pi \mathrm{P}+\pi \mathrm{X})$ varies dramatically with $\mathrm{P}_{\mathrm{T}}$ and that if this ratio is expressed as a function of $x_{R}=\left(p / p_{\max }\right)_{c m}^{T}$ it is nearly independent of energy.

Assuming the tripple Regge interpretation is successful, we expect to be able to obtain from these data a measurement of the $\rho$ and $A_{2}$ Regge trajectories over a large range of $t$ (out to $t \approx-3 \mathrm{GeV}^{2}$ ) thus supplementing our results from the charge exchange experiwent. These results could provide a crucial challenge to the "conotituent iliburchange model," according to which the $\rho$ trajectory should not go below -1 .

$$
* * * * * * * * * * * * * *
$$

Title of Project: The Crystal Ball Experiment at SPEAR

Person in Charge: C. Peck 
CALIFORNIA INSTITUTE OF TECHNOLOGY

Pasadena, California

Scientific and Professional Staff: C. Peck, R. Partridge, P. Chen

Scope of Work

This experiment to investigate $\gamma$ production in $e^{+} e^{-}$collisions at SPEAR using a "Crystal Ball" detector is a collaboration between. groups at Stanford, Harvard, and Caltech.

It is known that in $\mathrm{e}^{+} \mathrm{e}^{-}$collisions, about half of the total energy appears in the final state as neutral particles. At present, this large component is virtually unexplored and this experiment is designed to help fill this gap. The crystal ball is basically a spherical shell of sodium iodide, $40 \mathrm{~cm}$ inside diameter and $112 \mathrm{~cm}$ outside dimmeter, segmented into 630 optically separated triangular prisms, each 14 radiation lengths thick. Because of the good energy and angular resolution with which this apparatus will measure $\gamma$ 's, we expect to be able to reconstruct a large fraction of the produced $\pi^{\circ}$ 's.

The principal work done in this program has been in equipment design and evaluation and in the development of computing algorithms to sort out the number, direction, and energy of $\gamma^{\prime}$ s from the pulse heights obtained in a given event. The work on hardware has included the evaluation of many different phototubes for the crystal ball and various calibration light source schemes. A phototube meeting our requirements has been tentatively selected, but considerably more work remains to be done on the calibration problem.

Goals and/or Major Results to Date

It is not expected that these measurements will display characteristics much different from the already known inclusive spectrum of charged pions, but their reconstruction makes the apparatus particularly: sensitive to the discovery of unique energy photons which may be associated with $\psi$ decays. The apparatus will also be able to measure the total final state energy with fairly good resolution, and so should be able to discover processes involving high energy neutrinos in the final state.

$$
* * * * \star * * * * * * \star * *
$$

Title of Project: Bubble Chamber Program

Person in Charge: C. Peck

Scientific and Professional Staff: V. Davidson, F. Nagy, W. Ochs, C. Peck, C. Rosenfeld, A. Sheng 


\section{CALIFORNIA INSTITUTE OF TECHNOLOGY}

Pasadena, California

Scope of Work

This group is completing the analysis and interpretation of data obtained in two major "triggered" bubble chamber exposures at SLAC and smailer $300 \mathrm{GeV}$ pp exposures at Fermilab. This work is nearly complete and no further bubble chamber experiments are planned at this time.

For the $14 \mathrm{GeV} \pi^{ \pm} \mathrm{p}$ experiments from SLAC, all measuring, data reduction, event selection, efficiency calculation, and cross section normalization work has been completed. Three students are in the process of preparing these based upon these data. The reactions being emphasized in these respectively are, $\pi^{-} p+\pi^{-} \pi^{-} \Delta^{+}, \pi^{ \pm} p \rightarrow \pi^{ \pm}$ $\left(\mathrm{p} \pi^{\circ}, \mathrm{n} \pi^{+}\right)$, and $\pi^{ \pm} \mathrm{p} \rightarrow \pi^{ \pm} \pi^{+} \pi^{-}\left(\mathrm{p} \pi^{\circ}, \mathrm{n} \pi^{+}\right)$. It is expected that this work will be completed in the coming year.

Goals and/or Major Results to Date

During the past year, an interesting analysis of some of these data was published by Wolfgang Ochs. In it, it is shown that the amplitude for $\pi^{ \pm} p \rightarrow \pi^{ \pm}\left(p \pi^{\circ}, n \pi^{+}\right)$approximately factors into two parts, one depending only on the transverse motion, and the other, only on longitudinal motion.

It has been observed that the $\rho / \pi$ ratio increases with increasing transverse momentum. We searched for this effect in a sample of high multiplicity events ( $>18$ prongs) which were completely measured, and we find a narrow enhancement at the $\rho$-mass in the $\pi^{+} \pi^{-}$mass spectrum when the transverse momentum of the pair is greater than $0.6 \mathrm{GeV} / \mathrm{c}$. Since the enhancement is abnormally narrow compared to the known $p$-width, it is not clear that this observation is related to that seen at lower energies. However, the effect seems statistically significant and has been published.

$$
* * * * * * * * * * * * *
$$

Title of Project: Theory Group

Person in Charge: M. Gell-Mann, S. Frautschi, F. Żachariasen

Scientific and Professional Staff: M. Gell-Mann, G. Farrar, S. Frautschi, F. Zachariasen, G. Zweig, J. Schwarz, P. Ramond, B. Hasslacher, H. Fritzsch, P. Minkowski

Scope of Work and Major Results to Date

The theory group has been studying various facets of the gauge theories of quarks, leptons, and vector mesons. 


\section{CALIFORNIA INSTITUTE OF TECHNOLOGY}

Pasadena, California

Harald Fritzsch, Murray Ge11-Mann, and Peter Minkowsk1 worked out consequences of "vector-like" models of the weak interaction. After observing that neutrinos are likely to acquire a mass of order $10 \mathrm{eV}$ in these models, they pointed out major cosmological consequences: the blackbody 'neutrinos left over from the big bang could supply enough "missing mass" to close the universe and, clumped together in galactic clusters, could supply the missing gravitational potential needed to contain the kinetic energy observed for galaxies in clusters. Given more speculative assumptions, neutrino beams would exhibit oscillations similar to those observed in neutral $\mathrm{K}$ meson beams. One can search for these oscillations in neutrino beams from reactors or accelerators, and they might reduce the apparent neutrino flux from the sun. In addition to these cosmological applications, many detailed consequences of "vectorlike" models for particle physics were worked out.

Fred Zachariasen, with Peter Carruthers at Los Alamos, has investigated the properties of the Pomeron in gauge models by calculating multiple gluon exchange at large $s$ and small $t$.

Glennys Farrar and Steve Frautschi have made a speculative application of the Brodsky-Farrar scaling laws to the ratio of inclusive reactions $(a+b \rightarrow \gamma+X) /(a+b \rightarrow \pi+X)$ at large transverse momenta. The ratio is predicted to increase with momentum transfer because the pion has two constituents whereas the photon is thought to be elementary. The associated virtual photons are also predicted to be numerous; their decay into lepton pairs can then explain the "prompt lepton puzzle."

John Schwarz and Pierre Ramond have classified the possibilities for new string models based on graded Lie, algebras, with the conclusion that no new possibilities exist within the usual framework.

$$
* * * * * * * * * * * * * *
$$

Title of Project: Phenomenology Group

Person in Charge: G. Fox

Scientific and Professional Staff: R. Field, P. Stevens

Scope of Work

Experiments are hard, theories are abstruse and an uncontroversial confrontation between the two is rarely possible. In the consequent era of specialization, a phenomenologist tries to maintain rather 


\section{CALIFORNIA INSTITUTE OF TECHNOLOGY}

Pasadena, California

more than a smattering of knowledge of both theory and experiment. New insight is often attainable by comparing our superficially unspeaking giants: modelmaking is an important technique in this necessarily indirect process. The scope of this work is best defined by examples. This year saw a study of the important experiments with the (unique) polarized proton beam at Argonne. Collaboration with the theory (Feynman) isolated impressive agreement between the simple quark model and high transverse momentum hadron collisions. Currently another major effort is to understand if the experiments on inelastic electron and neutrino scattering show any evidence for the new theoretical idea of an asymptotical1y free theory. We have aloo made substantial contributions to the planning and computer software of two of the current Caltech experiments at Fermilab. Data which should help to elucidate and unify quark and Regge theories has been taken this summer (Caltech collaborative experiments \$260 and \#350). We plan to play a major role in the analysis of these experiments. An important part of the phenomenology effort is a collaboration with Berkeley in setting up a world wide data compilation system which uses the currently most sophisticated techniques in information retrieval.

R. D. Field and D. Novoseller work on the theoretical side of phenomenology, K. Yung on experimental work and P. R. Stevens is in charge of the compilation effort. G. Fox contributes to all parts of the effort which interact fruitfully with each other.

Goals and/or Major Results to Date

The goal of the phenomenology effort is to advance the theoretical understanding of high energy physics through analysis of experimental data. This goal is realized by having a balanced group with expertise in theory, experiment and compilation.

$$
\text { *************** }
$$


CARNEGIE-MELLON UNIVERSITY

Pittsburgh, Pennsylvania

Contract No. $\quad$ EY-76-C-02-3066

Title of Project: X-rays from Exotic Atoms

Person in Charge: R. Sutton

Scientific and Professional Staff: R. Button

Scope of Work

Antiprotons or $\mathrm{K}^{-}$mesons are stopped in appropriate targets and the $x$-rays emitted as the particle makes transitions between the various atomic states are observed with germanium or sllicon solid detectors. The intensity, energy and width of the $x$-ray lines are observed. From these data information concerning the strong interaction between the particle and the nucleus can be inferred as well as information about the properties of the particles themselves. An added feature of the $\mathrm{K}^{-}$experiments is that $\Sigma^{-}$particles can be emitted when the $\mathrm{K}^{-}$are finally captured by the nucleus. Thus, $x$-rays from $\Sigma^{-}$atoms can also be studied with the resultant information on properties of the $\Sigma^{-}$and its interaction with the nucleus.

The current emphasis of this work is investigation of the $\bar{p}-p$ and $\mathrm{K}^{-}-\mathrm{p}$ interaction. $\overline{\mathrm{p}}$ or $\mathrm{K}^{-}$are stopped in a liquid or gaseous hydrogen target and the resulting $x$-rays observed. A preliminary run in the spring of 1976 has given us hope that the $\bar{p}-p$-ray corresponding to the $2 \mathrm{p}-1 \mathrm{~s}$ and $3 \mathrm{p}-1$ s transitions can be observed.

Goals and/or Major Results to Date

Major results to date:

1) First measurement of the magnetic moment of the antiproton: $\mu_{-}=(-2.819 \pm 0.056)$ nuclear magnetons. (Phys. Rev. D $\underline{12}, 1232$ (1975)).

2) First measurement of the magnetic moment of the $\Sigma^{-}: \mu_{\Sigma^{-}}=$ $(-1.48 \pm 0.37)$ nuclear magnetons. (Phys. Rev. D $\underline{12}, 1232$ (1975)).

3) Most precise measurement of the antiproton mass: $\mathrm{m}=$ $938.236 \pm 0.048 \mathrm{MeV}$ (to be published).

4) Energies and widths of pertinent $x$-ray lines from $12 \bar{p}$ atoms, spanning the periodic table, have been measured, from which the level shifts and widths due to the strong interaction have been deduced. These data have all been fitted with a phenomenological potential of 


\section{CARNEGIE-MELLON UNIVERSITY}

\section{Pittsburgh, Pennsylvania}

the form $V=-\rho(r) \bar{a}$ where $\rho(r)$ is the nucleon density and $\bar{a}$ a complex scattering length. The value determined for $\bar{a}$ is $(0.85 \pm$ $0.38)+(2.66 \pm 0.28)$.

Goals :

1) To investigate the zero energy s-wave $k^{-} p$ and $\bar{p}-p$ interactions by studying the $x$-rays from $K^{-}$and $\bar{p}$ stopping in hydrogen. It is possible that $\Sigma^{-}$p $x$-rays can also be observed.

2) BNL is planning an improved low energy stopping beam (LESB II). We have indicated our intention of proposing a re-measurement of the $\Sigma^{-}$magnetic moment with the goal of obtaining an accuracy of \pm 0.1 nuclear magnetons. This precision would provide a meaningful check on the SU 3 prediction of 0.88 nuclear magnetons.

$$
\star \star \star \star * * * \star \star \star \star \star * * * *
$$

Title of Project: Theory

Person in Charge: R. Cutkosky, L. Wolfenstein

Scientific and Professional Staff: R. Cutkosky, L. Wolfenstein, R. Hendrick, M. Levine, L. Li, J. Donaghue

Scope of Work

The main efforts of the theory group have been in the phenomenology of strong and weak interactions and in quantum electrodynamical calculations. Wolfenstein and collaborators have studied theories of CP violation, the structure of weak currents, and parity-violating forces in nuclei. Cutkosky and collaborators have developed and applied new methods for determining scattering amplitudes and the properties of resonant states from experimental data. Levine has developed techniques for symbolic computation and applied them to QED calculations. The group also studies the properties of quantum field theory and models of hadronic structure.

Goals and/or Major Results to Date

Significant contributions to understanding of weak interactions have been made by Wolfenstein, especially in the analysis of CP violation (and development of the superweak theory) and in the analysis of the effects of weak currente.

Levine has developed powerful symbolic computation programs and applied them to ploneering calculations of $g-2$ for the electron and muon. 
CARNEGIE-MELLON UNIVERSITY

Pittsburgh, Pennsylvania

Methods for data analysis which emphasize analyticlty have been developed by Cutkosky and collaborators and applied to pion-nucleon scattering data; on the basis of their new information about excited states of nucleons, they have proposed an altered model of the nucleon's internal structure.

$$
* * * * * * * * * * * * * *
$$

Title of Project: Bubble Chamber Experiments

Person in Charge: A. Engler, R. Kraemer

Scientific and Professional Staff: S. Barish, S. Dado, A. Engler, G. Keyes, T. Kikuchi, R. Kraemer

Scope of Work

The bubble chamber group has pursued studies of strong and weak interaction phenomena. Two aspects of strong interactions have been investigated: 1) Properties of meson and baryon resonances, which are essentially "low-energy" phenomena ( $\leq 10 \mathrm{GeV})$; and 2 ) Inclusive reactions, above $100 \mathrm{GeV}$. Meson resonances were studied in the reaction $\pi^{+} \mathrm{d}$ at $\mathrm{GeV} / \mathrm{c}$ from an exposure of $30^{\prime \prime}$ bubble chamber at ANL. This work has been completed. Strange baryon resonances were studied in formation experiments of $\mathrm{K}^{\circ} \mathrm{p}$ using the ANL $\mathrm{K}^{\circ}$ beam. We are currently studying $\Delta$ and $\mathrm{N}^{*}$ formation in $\mathrm{pp}$ colfisions using the polarized proton beam at ANL at 6 and $12 \mathrm{GeV} / \mathrm{c}$. Inclusife reactions, such as $\mathrm{p}+\mathrm{n} \rightarrow \mathrm{p}_{\text {slow }}+$ anything are studied in $\mathrm{pd}$ and $\pi \mathrm{d}$ collisions at $100 \mathrm{GeV} / \mathrm{c}$ and $\mathrm{pd}$ collisions at $400 \mathrm{GeV} / \mathrm{c}$. We are using the 30" bubble chamber-MPWC hybrid system at Fermilab. In our studies of weak interactions, we are pursuing the following topics: study of form factors of $\mathrm{K}_{\ell 3}^{\circ}$ and $\mathrm{K}_{3 \pi}^{\circ}$ decays using the ANL monochromat $1 \mathrm{c} \mathrm{K}_{\mathrm{O}}^{\circ}$ beam. This work is nearing completion. Most of our current and future efforls will be dedicated to the study of neutrino reactions at ANL and Fermilab energies. We obtained data $(60 \mathrm{~K}$ pictures) from the 15' bubble chamber filled with $\mathrm{H}_{2}$ at Fermilab. Additional data (140 $\mathrm{K}$ pictures) using $\mathrm{H}_{2}$ in the $15^{\prime}$ chamber is expected before the end of this year, $300 \mathrm{~K}$ picutres in $D_{2}$ during 1977. In addition, we expect to obtain $10^{6}$ pictures in the $12^{\prime}$ bubble chamber filled with $\mathrm{D}_{2}$ at $\mathrm{ANL}$. The neutrino experiments are performed in collaboration with groups at ANL and Purdue University.

Goals and/or Major Results to Date

Major results to date: 
In our studies of meson resonances we have obtained the first conclusive evidence of the existence of the decay mode $f \rightarrow 4 \pi$. We also obtained for the first time evidence for the decay mode $\omega(1675) \rightarrow \omega \pi \pi$. We have studied the reactions $K_{L}^{0} p \rightarrow \Lambda \pi^{+}, \Sigma^{o} \pi^{+}$and $\mathrm{K}_{\mathrm{S}} \mathrm{p}$ and have been able to rule out several partial wave solutions of the KN and $\bar{K} N$ system.

In our studies of $\bar{v}_{p}$ interactions at Fermilab energies, we have been for the first time able to study the semi-inclustve reaction $\bar{v} p \rightarrow \mu^{+}+\pi^{-}+$anything and find that the quark parton model provides a good representation of the data.

$$
* * * * * * * * * * * * * *
$$

Title of Project: Counter Group

Person in Charge: R. Edelstein

Scientific and Professional Staff: R. Edelstein, D. Green, H. Halpern, E. Makuchowski, J. Russ

\section{Scope of Work}

In the past few years we have helped build the Brookhaven Multiparticle Spectrometer, providing a substantial amount of the hardware for it. With its very large geometrical acceptance it should be a good facility for searching for and studying new resonances. In our own current experiment we study the details of several two body scattering channels related through baryon exchange in order to measure the effect of absorption in these processes and thus distinguish between competing theoretical models. In particular we hope to discover striking structures in the angular distributions for reactions induced by antiproton beams, a field in which much remains to be done.

\section{Goals and/or Major Results to Date}

The most outstanding achievement of the group in the past was the discovery, in collaboration with the Collins group at Brookhaven, of the phenomenon of Diffraction Dissociation in Reactions of the type $\mathrm{pp} \rightarrow \mathrm{pN}$ and $\pi \mathrm{p} \rightarrow \pi \mathrm{N}^{2}$. This demonstrated the inclmate connection between elastic scattering and certain closely related particle production channels in high energy scattering. In a recent experiment using nuclear targets, we have studied the related process of the interaction of these produced systems, $N^{*}$ 's, with nuclear matter. We find the total cross section $\sigma_{\mathrm{T}}\left(\mathrm{N}^{*}, \mathrm{~N}\right)$ decreases dramatically as the mass of the $\mathrm{N}$ increases (A factor of 2 from $1.3-1.8 \mathrm{GeV}$ ). 
HARVARD UNIVERSITY

Cambridge, Massachusetts

Contract No. $\quad$ EY-76-C-02-3064

Title of Project: Study of Elastic Neutrino-Proton Scattering

Person in Charge: C. Rubbia

Scientific and Professional Staff: C. Rubbia, L. Sulak, W.

Kozaneski, J. Strait

Scope of Work

Evidence for a neutral weak current has been discovered in neutrino scattering. Although the nature of this neutral current is fundamental to the understanding of the weak interaction and its possible unification with electromagnetism and the strong interaction, its basic properties remain unknown: What is the space-time structure of the hadronic neutral current? Does it exhibit vector-axial vector interference (parity violation)? Is it consistent with the gauge theories that unite weak interactions and electromagnetism?

Experiment 613 at Brookhaven National Laboratory addresses these questions by studying the simplest neutral current reactions: $\nu p \rightarrow \nu p$ and $\bar{p} \rightarrow \bar{p}$. These reactions are neutral current analogs of classic charged-current beta decay. The space-time structure of the neutral current is directly unfolded from the energy-angle distribution of the recoil proton. Any difference between neutrino and antineutrino reactions is a direct signature of parity violation in the hadronic neutral current. Elastic neutrino-proton scattering had been previously impossible to observe because of low rates and high background from neutron-induced reactions and reactions with unidentified secondaries. These difficulties have been overcome with a new type of target-detector 1) with a large mass (30T) to insure high rate, 2). with active shielding (the entire target is liquid scintillator) to detect neutrons by their multiple interactions, 3) with large dimensions ( $3 \mathrm{~m} \times 3 \mathrm{~m} \times 6 \mathrm{~m}$ ) to stop and identify protons, pions, muons, and photons, and 4) with live time recording to recognize the time signature of the $\pi \rightarrow \mu \rightarrow$ e decay sequence and to reject slow neutrons that are out of time relative to neutrinos which travel at c. Drift chambers (4m $x 4 \mathrm{~m})$ are interspersed within the detector to record the trajectories of charged particles. A large aperture magnet $(2.5 \mathrm{~m} \times 2.5 \mathrm{~m})$ after the detector measures the momenta of muons produced in charged-current reactions. These reactions provide a normalization for the neutral current channels and a measurement of the neutrino flux. 


\author{
HARVARD UNIVERSITY \\ Cambridge, Massachusetts
}

Goals and/or Major Results to Date

This experiment has discovered $v_{p} \rightarrow v_{p}$ and $\bar{v}_{p} \rightarrow \bar{v}_{p}$ scattering. It has also observed the charged-current forms of these reactions,.. $\nu n \rightarrow \mu^{-} p$ and $\bar{v}_{p} \rightarrow \mu^{+} n$, and measured the ratios of the neutral and charged processes. A striking difference has been observed between the neutrino and antineutrino reactions, implying that a pure vector or axial vector interaction (non-parity violating) is unlikely. Further, the neutral current rates, the recoil proton spectra, and the particle-antiparticle difference are entirely consistent with the unifying theories.

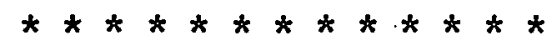

Title of Project: Study of Deep Inelastic Neutrino Interactions at Fermilab

Person in Charge: C. Rubbia

Scientific and Professional Staff: C. Rubbia, L. Sulak, P. McIntyre, J. LoSecco, J. Rich

Scope of Work

This series of experiments is studying the structure of the nucleon at very small distances $\left(\sim 10^{-14} \mathrm{~cm}\right)$ and the nature of the weak. interaction via deep inelastic neutrino-nucleon scattering. They utilize a massive (100T), totally active (1iquid scintillator) target detector to obtain information at the highest $q^{2}$ 's (smallest distances). A large acceptance muon spectrometer identifies any muons produced in the interactions.

Goals and/or Major Results to Date

1) $\nu \mathrm{N} \rightarrow \mu \mathrm{X}$. We have confirmed the parton model of the nucleon for neutrino energies below $30 \mathrm{GeV}$. However, above $30 \mathrm{GeV}$, the reaction $\nu N \rightarrow \mu X$ shows dramatic deviations from scaling. These scaling violations appear at high energy transfer $y$ and low scaling variable $X$, precisely the kinematic region expected for new particle production in the hadronic channel. The deviation has been shown to violate charge symmetry; this has occurred in the past when a new quantum number is produced in the interaction. Further, the invariant mass of the events in the region of the violation reveal an excess above that produced by the electromagnetic interaction in analogous deep inelastic electron scattering, suggesting that the particle is unique to the weak interaction. The enhancement in the invariant mass distribution peaks at $\sim 3 \mathrm{GeV} / \mathrm{c}^{2}$, yielding a measure of the mass of new particle. 
HARVARD UNIVERSITY

Cambridge, Massachusetts

2) $\nu \mathrm{N} \rightarrow \mu \mu \mathrm{X}$. We have discovered neutrino-induced events in which two muons are observed in the final state. The characteristics of these events suggest new particle production at the hadronic vertex with subsequent beta decay of the particle. Invariant mass and transverse momentum distributions suggest a mass of $\sim 3 \mathrm{GeV} / \mathrm{c}^{2}$. The weak decay of the new object indicates production of a new quantum number. Some of the events, however, involve equal sign dimuons, the origin of which is still unclear.

3) $\quad v N \rightarrow v X$. The experiment has also discovered events with no muons in the final state, indicative of a neutral current interaction. Further, we have shown that this new interaction is parity violating.

The current experiment (\$310) has improved the muon acceptance of the detector by a factor of 4 . This will make the highest $\mathrm{q}^{2}$ accessible for reaction 1) will allow the study of muon pairs in 2) with acceptance to very low muon energies, and will give $>99 \%$ acceptance for possible missing muons in reaction 3). Also, the mass of the target has been increased by a factor of 4 to attempt to discover $\nu N \rightarrow \mu \mu \mu \mathrm{X}$ events if they exist. To obtain full kinematic reconstruction of reaction 3 ), the detector has been modified to measure the angle as well as the energy of the hadronic shower.

$$
* * * * * * * * * * * * * *
$$

Title of Project: Muon Scattering at Fermilab

Person in Charge: R. Wilson

Scientific and Professional Staff: R. Wilson, W. Loomis, A. Sessoms, B. Gordon, D. Shanbroom

Scope of Work

The muon scattering facility at Fermilab is being used in an ongoing program to study lepton hadron scattering at high energies. Data collection from the first series of experiments (E98) was completed in 1975 and the results are discussed briefly below. This experiment studied both muon-hydrogen and muon-deuterium scattering in the energy range of 100 to $150 \mathrm{GeV}$.

In the present experiment, E398, several new features have been added to the experiment. The largest is a Cerenkov counter which will allow the identification of charged pions, K-mesons and protons using pulse height analysis. In addition, a set of three cylindrical drift chambers with two-dimensional readout have been constructed. These chambers 
HARVARD UNIVERSITY

Cambridge, Massachusetts

surround the liquid hydrogen/deuterium target, and will allow a measurement of multiplicities and a detailed study of the recoil proton spectrum to be made. We are also running at $225 \mathrm{GeV} / \mathrm{c}$ incident muons to get to higher $\mathrm{q}^{2}$.

Future experiments will study shadowing effects in virtual photons on heavy nuclei and will search for missing energy in mu pair production.

\section{Goals and/or Major Results to Date}

The principal result from the first series of muon scattering experiments has been a confirmation of violations of scaling in inelastic lepton-nucleon scattering already found at Fermilab.

The results, combined with SIAC data, show that $\delta_{0}^{1} v w_{2}\left(q^{2}, v\right) d x$ remains constant to $5 \%$ (scales) as $q^{2}$ varies from 1 to $30 \mathrm{GeX}$. On the other hand, at high $x$ (low $\omega \equiv 2 \mathrm{Mv} / \mathrm{q}^{2}$ ) $v_{2}$ falls with $\mathrm{q}^{2}$ about $30 \%$ over this region, and at low $x$ (high $w$ ) $v w_{2}$ rises about $20-30 \%$. These results are in agreement with theoretical ideas of asymptotic freedom, whereby partons radiate gluons in much the same way as electrons radiate photons, leading to a behavior violating scaling similar to that of radiative corrections but with a parton gluon coupling to be determined.

We have shown that over a wide range of $\nu \& q^{2}$, the hadron spectra show similar structure; we also have shown that although an exclusive channel - $\mu \mathrm{P} \rightarrow \mu \rho^{0} \mathrm{P}$ - follows a behavior suggested by vector dominance quite closely, the inclusive $\rho^{\circ}$ channel follows the hadron spectra.

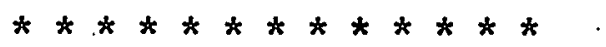

Title of Project: Scattering from a Polarized Target

Person in Charge: F. Pipkin

Scientific and Professional Staff: F. Pipkin, M. Law, W. Johnson, R. Kline

Scope of Work

In collaboration with groups from Berkeley, Argonne, and Yale we are carrying out an experiment with a polarized target at Fermilab. The Harvard group was responsible for the forward arm. The largest 


\section{HARVARD UNIVERSITY. \\ Cambridge, Massachusetts}

components are the two threshold Cerenkov Counters used to identify pions, kaons, and protons. The experiment employs magnetic analysis in both the forward and recoil arms and a set of proportional wire chambers is used to determine the trajectory of the forward and scattered particles. This gives a tight constraint and allows us to reject the quasielastic scattering from the carbon in the target. The purpose of the experiment is to survey the polarization at the high energy given by Fermilab.

Goals and/or Major Results to Date

To date we have made measurements at $100 \mathrm{GeV} / \mathrm{c}$. The $\pi^{+}$and $\pi^{-}$ display the mirror like symmetry observed at lower energies and continue the $1 / \sqrt{ }$ s decrease in polarization. The proton results show a similar behavior at low momentum transfer but are more problematic at high $t$.

$$
* * * * * * * * * * * * *
$$

\section{Title of Project: Electroproduction Studies}

Person in Charge: F. Pipkin

Scientific and Professional Staff: F. Pipkin, S. Raither, M. Herzlinger, S. Holmes, C. Bebek

Scope of Work

Two magnetic spectrometers operated in coincidence have been used at the Wilson Synchrotron Laboratory at Cornell University to study the final state hadrons in electroproduction reactions. One spectrometer is used to observe the scattered electron and the other spectrometer is used to observe one or more of the hadrons. In the past this apparatus has been used to study exclusive pion production, inclusive pion production, exclusive kaon production, inclusive kaon production and inclusive proton production. The data have been used to determine the pion form factor up to $\mathrm{Q}^{2}=10.0 \mathrm{GeV}^{2}$, to test the high momentum transfer predictions of the constituent interchange model, to study the behavior of the invariant structure function for $\pi^{ \pm}, \mathrm{K}^{+}$ and $p$, to test the quark parton model predictions for the $\pi^{+} / \pi^{-}$ ratio, to separate the longitudinal and transverse components in both exclusive and inclusive reactions, and to study $\phi$ electroproduction. 
HARVARD UNIVERSITY

Cambridge, Massachusetts

Goals and/or Major Results to Date

Our chief goals are to understand the final states in electroproduction reactions and to see if one can see the footprints of the constituents in the final state hadrons. The major results are the measurement of the pion form factor, the evidence $f^{n}$ favor of the constituent interchange model, the evidence from $\pi^{+} / \pi^{-}$ ratios for the predictions of the quark parton model, the observed behavior with $Q^{2}$ of the $\pi^{+}$and $\pi^{-}$invariant structure functions, the separation of the longitudinal and transverse components and the test for shrinkage in $\phi$ electroproduction.

$$
\star \star \star \star * \star * * * * * * * *
$$

Title of Project: Muon-Pair Production at the CERN-ISR

Person in Charge: $K$. Strauch

Scientific and Professional Staff: K. Strauch, H. Newman

Scope of Work

The aim of this experiqent is to study the reaction $p+p \rightarrow$ $\mu^{+}+\mu^{-}+x^{++}$(where $x^{++}$stands for all possible states) at the highest available energy $(\sqrt{s}=56-60 \mathrm{GeV})$ over a much wider angular range than has been studied before. According to present ideas, muon pairs are produced in this reaction primarily through the process $q+\bar{q} \rightarrow \gamma_{v} \rightarrow \mu^{+}+\mu^{-}$where $q$ and $\bar{q}$ are quark and antiquark constituents of the interacting protons, and $\gamma_{\mathrm{y}}$ is an intermediate virtual photon with the invariant mass of the muon pair. Observation of the mass and angular distributions of the muon pair thus gives information on the quark-antiquark content of the proton such as the momentum distribution inside the proton. Any high mass peak in the pair mass spectrum would Indicate the presence of a new vector boson. The experiment covers the 2-50 GeV mass region with an average resolution of $18 \%$ (FWHM) and detects muons between $15-110^{\circ}$.

The ISR Interaction region is surrounded with turoldal colls consisting of magnetized steel. Trigger counters and drift chambers are imbedded and surrround the magnets; they serve to identify muons through their penetration and to measure their momentum. A central set of drift chambers measures the muon direction, and that of other charged particles produced in the same interaction. 


\section{HARVARD UNIVERSITY \\ Cambridge, Massachusetts}

Goals and/or Major Results to Date

The experiment will be installed before the end of calendar 1976 and is scheduled for data taking in the following year.

$$
* * * * * * * * * * * * *
$$

Title of Project: The "Crystal Ball" Experiment at SLAC

Person in Charge: K. Strauch

Scientiflc and Professional Staff: K. Strauch, W. Kollman, M. Richardson

Scope of Work

Because of the relative simplicity of the one-photon intermediate state, high energy $\mathrm{e}^{+} \mathrm{e}^{-}$annihilation has proven to be extraordinarily useful in increasing our understanding of elementary particles and basic interactions. Relatively little is known about the nature of properties of that fraction of the available energy which is emitted as neutral particles. This experiment is specifically designed to study that major portion of the neutral energy which is observed as photons. 672 triangular modules of $\mathrm{NaI}$, assembled into an Icosahadron which approximates a sphere, serve to measure the energy and position of photons and perform hadron calorimetry. A central cavity contains spark and proportional chambers to identify charged particles and to measure their energy.

NaI was chosen as the active material because of its excellent photon efficiency and energy resolution over a wide spectrum including the low energy end which is likely to be particularly interesting.

Goals and/or Major Results to Date

The physics which is anticipated to be studied over a wide energy range includes:

1) All neutral tinal states using an energy trigger;

2) The new photon spectroscupy of charmed states with better resolution and higher sensitivity;

3) The nature of neutral particles emitted; 
HARVARD UNIVERSITY

Cambridge, Massachusetts

4) Inclusive spectra of photons, $\pi^{\circ}$ and $\eta^{\circ}$ mesons;

5) A search for heavy leptons decaying electromagnetically; and

6) A search for unexpected phenomena with a detector very different from those used before.

Data taking is scheduled to start in FY 78.

$$
* * * * * * * * * * * * * *
$$


UNIVERSITY OF ILLINOIS

Urbana, Illinois

Contract No. $\quad$ EY-76-C-02-1195

Title of Project: Experimental Elementary Particle Physics

Person in Charge: A. Wattenberg

Scientific and Professional Staff: G. Ascoli, R. Brown, J. Butler, J. Cooper, B. Eisenstein, J. Elliott, W. Francis, G. Giladding, I. Holloway, T. Kirk, L. Koester, Jr:, U. Kruse, R. Messner, T. O'Halloran, C. Pang, R. Sard, M. Shupe, A. Wattenberg, R. Downing, N. Meyer, T. Noggle, B. Schreiber, J. Simaitis, J. Wray

\section{Scope of Work}

The High Energy Physics Experimental Program at the Urbana campus of the University of Illinois uses a variety of detecting techniques at FNAL, BNL, and ANL. There are three experimental programs at Fermilab: one uses a broad band neutral beam combined with a multiwire proportional spectrometer, an electromagnetic shower detector, a hadron calorimeter, and a muon identifier. Neutron and photon beams have been used to study the production of new particles as well as the dynamics of $\mathrm{J} / \Psi$. The second FNAL program uses a magnetic spectrometer based on the Chicago Cyclotron facility. One set of experiments studies the scattering of muons and the accompanying products. In other experiments scheduled to begin this coming winter, the detector is being supplemented, and a pion beam will be used to study the pion production of charmed particles and multipion states. The third program of experiments at FNAL has used a bubble chamber combined with a proportional chamber tagging facility which was built by Illinois and its collaborators. The Illinois involvement in the bubble chamber collaboration will be terminated after the analyses of the present runs are completed.

At BNL a counter hodoscope system and an optical spark chamber detector are being used with neutrino and antineutrinos to s tudy neutral currents and to look for multiple lepton production.

At ANL a streamer chamber program is being completed which has been studying the backward production of mesons.

\section{Goals and/or Major Results to Date}

1) In the wide band photon beam experiments at FNAL there is evidence for the production of a new antibaryon which decays into $\bar{\Lambda} \pi^{-} \pi^{-} \pi^{+}$with $M=2.26 \pm .01 \mathrm{GeV} / \mathrm{c}^{2}$. There is al so evidence of a 


\section{UNIVERSITY OF ILLINOIS \\ Urbana, Illinois..}

higher mass state with 4 pions which decays into this $2.26\left(\mathrm{GeV} / \mathrm{c}^{2}\right)$

state. The 2.26 resonance is consistent with the theoretical

predictions for the lowest mass charm baryon state.

2) We have observed $10 \mathrm{~W}^{+} \mu^{-}$events in the mass region $4.7 \pm 0.2 \mathrm{GeV} / \mathrm{c}^{2}$ produced by photons. There is a very low probability that half of these events can be accounted for by Bethe-Heitler processes.

3) In the neutron experiments we find low limits for the production of $\Psi^{\prime}$, and for the production of charm particles arcompanying $\mathrm{J} / \Psi$ particle production.

4) From studying the dynamics of $\mathrm{J} / \Psi$ production by neutrons on a series of targets, we find that the yield of higher mass states varies almost directly as the number of nucleons in contrast to the $\rho+w$ whose production varies approximately as $A .62$.

5) From the results of our neutrino experiment at BNL, we were the first to report the observation of the fundamental process of the elastic scattering of neutrinos by protons, $\nu+p \rightarrow \nu+p$.

6) The production of $\mu$ e events by neutrinos has also been observed in the BNL experiment at a level that is in agreement with the results being obtained by other groups.

7) In the high energy inelastic muon scattering experiment at FNAL, measurements of $W_{2}$ for neutrons and protons at large values of $V(20 \rightarrow 130 \mathrm{GeV})$ and over a wide range of $q^{2}\left(0.5 \rightarrow 15 \mathrm{GeV} / \mathrm{c}^{2}\right)$ shows the Bjorken scaling behavior is maintained in this new kinematic region.

$$
* * * * * * * * * * * * * * *
$$


MASSACHUSETTS. INSTITUTE OF TECHNOLOGY

Cambridge, Massachusetts

Contract No, EY-76-S-02-2959

Title of Project: Particle Theory Group

Person in Charge: M. Deutsch

Scientific and Professional Staff: C. DeTar, B. Feld, H. Feshbach, K. Huang, R. Kackiw, R. Jaffe, K. Johnson, F. Low, C. Thorn, V. Weisskopf, J. Young, J. Goldstone, L. McLerran, F. Mansoni, T. Neff, C. Noh1, D. Peaslee, C. Rebbi, J. Willemsen

Scope of Work

The Laboratory.'s Theoretical Group is primarily concerned with

1) The structure of hadrons;

2) The fundamental interactions between hadrons; and

3) The weak and electromagnetic interactions between leptons and between leptons and hadrons as these are exhibited by and are responsible for the properties and interactions of elementary particles and nuclei.

It is, therefore, interested in the interpretation and understanding of the experimental data including the mass (or energy), spin, parity, isospin, G parity, etc. of the elementary particles and the ground and excited states of nuclei, the weak and electromagnetic decays of these systems as well as the interaction between them. Information on these questions is furnished by elastic and inelastic interactions between dynamical systems, including nuclei, hadrons, leptons and photons. As a consequence many of the theoretical considerations are conditioned by the studies which are possible at the older accelerators as well as the new facilities which have recently become operational such as Fermilab.

The scope of work of the Particle Theoretical efforts of the Center currently includes studies of hadron models, strong interaction Regge dynamics and a study of dynamical models of symmetry breaking in the context of field theory.

Goals and/or Major Results to Date

At the present time, the MIT Bag Model is still under intensive study. In particular, it is being applied to multiquark hadrons and mesons with a number of interesting predictions. The technology of the model is also under development with the intention of its 


\section{MASSACHUSETTS INSTITUTE OF TECHNOLOGY}

Cambridge, Massachusetts

application to the calculation of level widths. Charmed states are also under investigation; in particular, electromagnetic mass differences have been calculated.

The study of classical solutions of local field theories with applications to the quantum theory is also being intensively carried out. Of particular interest are states with unexpected quantum numbers.

Finally, the development of strong interaction phenomenology continues to be a concern of the group.

$$
\star * * * * \star * * * * * * * *
$$

Tille of Project: Counter Spark Chamber Program: Fermilab and PEP

Person in Charge: M. Deutsch

Scientific and Professional Staff: W. Busza, J. Friedman, H. Kenda11,

L. Osborne, L. Rosenson, D. Barton, G. Brandenburg, P. Dobrowolsk1,

B. Nelson, R. Verdier

Scope of Work

The Counter Spark Chamber Group is carrying out a program of investigations of strong interactions at high energies using the Fermilab Accelerator.

Using the single arm spectrometer facility designed and constructed by MIT and other institutions, we have made extensive measurements (E96) of elastic and low mass inelastic scattering of $\pi^{ \pm}, K^{ \pm}, P$, and $P$ from the proton. Some of the topics about which these measurements provide information are the following: The s-dependence of the elastic scattering slope parameters for the above particles; the relative opacities of $\pi$ mesons, $K$ mesons, and nucleons; the validity of various geometrical models, e.g., geometric scaling; the validity of factorization and Feynman Scaling; the size of the triple Pomeron coupling based on a triple-Regge analysis of the inelastic spectrum.

The group is currently using the spectrometer in conjunction with an array of vertex detectors to measure inclusive $\pi, K, p$, and $\bar{p}$ spectra from hadron proton scattering along with the associated charged particle multiplicities and pseudo-rapidities (E118). In addition to proviiding stringent tests of models describing high energy multiparticle production, this experiment will provide 


\section{MASSACHUSETTS INSTITUTE OF TECHNOLOGY \\ Cambridge, Massachusetts}

further tests of factorization as applied to multiplicity predictions and thus will provide information about the nature of the Pomeron.

In another research program (E178) the group has investigated particle production mechanisms by studying particle multiplicities and their rapidity distributions in hadron-nucleus collisions. The experimental results of this program have placed strong constraints on possible models of multiparticle production, in particular on the space-time development of the process. To answer many interesting questions suggested by results obtained so far a more detailed study of hadron-nucleus collisions, using the single arm spectrometer, has been proposed to and approved by the Fermilab (Exp. \#451).

\section{Goals and/or Major Results to Date}

The slope parameter of the diffraction peaks in the elastic scattering of pions, kaons, and antiprotons have been obtained with good precision from Fermilab E96. The elastic scattering data can be interpreted as indicating interaction radii for mesonnucleon scattering about 5-10 percent smaller than for nucleonnucleon interactions. Also, the former seems to be about 20 percent more transparent than the latter. The spatial distribution of the hadronic interaction density derived from these data agrees well with the electromagnetic pion form factors. Data from E118 are being evaluated and further data acquisition is scheduled during the remainder of 1976 .

$$
* * * * * * * * * * * * * *
$$

Title of Project: Counter Spark Chamber Program: Other Projects

Person in Charge: M. Deutsch

Scientific and Professional Staff: J. Friedman, J. Kendall, I. Osborne, L. Rosenson, M. Deutsch, P. Luckey, D. Barton, C. Bolon, B. Nelson, R. Verdier

Scope of Work

This program covers various projects undertaken by members of the counter-spark chamber program before consolidation of this group. All of these are near complction:

1) Investigation of nucleon structure by inelastic electron scattering at SLAC (Friedman, Kendall, Verdier). This involves final analysis of SLAC experiments $49 \mathrm{~b}$ and E87 on large angle $e-p$ and $e-d$ scattering. 
MASSACHUSETTS INSTITUTE OF TECHNOLOGY

Cambridge, Massachusetts

2) $\bar{p}$ interactions in the $T$ and $U$ meson region using visual spark chambers at BNL (Barton, Nelson, Rosenson). Angular distributions, as a function of energy, for neutral two-body final state decays $\left(\pi^{\circ}\right.$ and $\eta$ ). Total annihilation cross section as a function of energy. This experiment was designed to detect possible narrow resonances in the $\bar{p} p$ system.

3) Hadron electroproduction; SLAC experiment E97. (Osborne, Luckey, Bolon in collaboration with SLAC). Electroproduction of pions, kaons, protons, and neutral vector mesons from protons, neutrons and nuclei. This experiment yields 50 times more data than previouo measurements on pion production and provides particle identification for the other products.

4) Elastic photon-proton scattering. (Deutsch, in collaboration with Tufts; University of Massachusetts, Amherst; Corne11). This experiment, carried out at Cornel1, measures proton Compton effect cross sections for incident photons between 2 and $6 \mathrm{GeV}$ and up to $4.3(\mathrm{GeV} / \mathrm{c})^{2}$, well outside the diffraction region.

5) Detector development for large Fermilab neutrino detector (Osborne, Frisch, Luckey). Construction and testing of drift chamber modules considered suitable for very large arrays. Monte Carlo studies of such detectors.

Goals and/or Major Results to Date

1) Major conclusions from the electron scattering experiments are: the ratio of neutron to proton cross sections decreases monotonically as threshold is approached and reaches about 0.28 for $q^{2} / 2 \mathrm{M}=0.8$, close to the limit of 0.25 imposed by quark models. The longitudinal cross section is small and its dependence on energy loss is consistent with spin $1 / 2$ partons.

2) The angular distributions of $\pi^{\circ} \pi^{\circ}$ and $\pi^{\circ} n^{\circ}$ states in pp annihilation show much structure but a detailed search has not shown any narrow resonances. The same is true for the total annihilation cross section in the region of $0.5-2 \mathrm{GeV} / \mathrm{c}$ incident $\overline{\mathrm{p}}$ energy.

3) Final results of the electroproduction experiment are in preparation. Results on pion charge ratios are in excellent agreement with a simple quark model.

4) Compton scattering cross sections have been obtained together with $\pi^{\circ}$ photoproduction measured in the same experiment. The results are found to be larger than predicted by simple vector dominance or constituent models. 
MASSACHUSETTS INSTITUTE OF TECHNOLOGY

Cambridge, Massachusetts

Title of Project: Accelerator Physics Collaboration (APC) (Bubble Chamber Program)

Person in Charge: M. Deutsch

Scientific and Professional Staff: R. Hulsizer, V. Kistiakowski, I. Pless, R. Yamamoto, F. Barreiro, O. Benary, E. Hafen

Scope of Work

The APC program has two major components. The first is a comprehensive charge and energy dependent study of pion-proton interactions. The group has $4,6,8$, and $15 \mathrm{GeV} / \mathrm{c}$ pion film; the 4 and $6 \mathrm{GeV} / \mathrm{c}$ film from the ANL 30" chamber and the 8 and $15 \mathrm{GeV} / \mathrm{c}$ film from the 82" SLAC chamber. The main purposes of this work are cross channel analysis, trajectory isolation, absorption Regge and other model studies, resonance studies, and inclusive reaction studies.

The second component is utilization of the Fermilab 30 " bubble chamber PWC Hybrid System. This system permits the measurement of the particles in the forward, high momentum jet. Hence, one can make studies previously not possible. Among these are angular correlation measurements in the center of mass of particle groupings, $P_{||}$and $P_{\perp}$ correlations, invariant mass studies, leading particle studies, true rapidity studies, and charge correlation studies. This system is being improved by the addition of a lead glass photon detector which will make possible studies of channels with up to three $\pi^{\circ}$ mesons. The group is actively participating in the addition of an external particle identified to the system and intends to add an external muon identifier (EMI).

This group collaborates in the Fermilab program with the following groups: Brown University, Illinois Institute of Technology, University of Illinois (Urbana), Indiana University, Johns Hopkins, Rutgers-Stevens-Alban, Tennessee-ORNL, Yale-Fermilab, and the University of Padova. Collaborations with other European groups are under discussion: CERN, Nijmegen, Strasbourg, Rome, Cambridge, Tohoku, Tel-Aviv, Weizmann.

Goals and/or Major Results to Date

In the period July 1, 1975, to January 1, 1976, six papers for publication and nine abstracts to physical society meetings have been submitted. The six papers are of prime importance. Five papers report results from Fermilab Experiment 154, the initial exposure of the Fermilab PWC Hybrid Bubble Chamber System. The 


\section{MASSACHUSETTS INSTITUTE OF TECHNOLOGY}

Cambridge, Massachusetts

group has presented evidence for charged cluster emission, reported on $\rho$ production, 4 prong exclusive channels and presented our final results for this experiment concerning the 2 prong inelastic events and cross sections and charged multiplicity distributions for $\pi^{-} \mathrm{p}$ and $\mathrm{K}^{-} \mathrm{p}$ interactions at $147 \mathrm{GeV} / \mathrm{c}$.

One paper is a complete discussion of our two component model. This model is based on a cross channel analysis of data taken in the ANL $30^{\prime \prime}$ bubble chamber and has now been tested with PP and $\pi \mathrm{P}$ reactions over the momentum range of $4 \mathrm{GeV} / \mathrm{c}$ to $1500 \mathrm{GeV} / \mathrm{c}$.

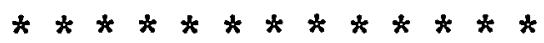

Title of Project: Electromagnetic and Other Interactions Program

Person in Charge: M. Deutsch

Scientific and Professional Staff: U. Becker, S. Ting, J. Burger, M. Chen, G. Everhart, F. Heimlich, T. Landerlung, D. Luckey, S. Sigamoto, W. Toki, S. Wu

Scope of Work

This group is engaged in several experiments primarily, but not exclusively, involving observation of lepton pairs to study high energy interactions and properties of vector mesons. Currently, the major activities are:

1) Final evaluation and publication of results from experiments following the discovery of the $\mathrm{J}$ particle with the precision double-arm spectrometer at BNL.

2) Construction and installation of apparatus for a major experiment (ISR 804) at CERN-ISR designed to observe the process $p+p \rightarrow \mu^{-}+x$ in a search for new vector particles with masses up to $60 \mathrm{GeV}$ and for other insights into the structure of weak and electromagnetic interactions.

3) A proposal for a large $4 \pi$ detector at the PETRA storage ring, designed to measure asymmetries in the process $e^{+} e^{-}+\mu^{+} \mu^{-}$, shuwing interference between weak and electromagnetic interactions, and to search for rare phenomena such as $e^{+} e^{-} \rightarrow \mu^{+} e^{-}+n v$.

4) Members of the group are participating with European collaborators in two experiments at CERN-ISR. One (ISK 410) is a study of inclusive pion production and of two-pion correlations in the split-field magnet; the other (ISR 803) is a study of low $\mathrm{p}_{t}$ pions. 


\section{MASSACHUSETTS INSTITUTE OF TECHNOLOGY \\ Cambridge, Massachusetts}

5) The group participates in experiments at DESY studying photoproduction of particle pairs with a double spectrometer similar to the one used at BNL.

Goals and/or Major Results to Date

Main results obtained at $B N L$ after discovery of the $\mathrm{J}$ particle:

1) A search for new states in $K \pi, K p, K \bar{p}$, with mass resolution of $5 \mathrm{MeV}$ shows no new states in the mass region $1.5-5 \mathrm{GeV}$ to limits of $\sigma B=10^{-32}-10^{-35} \mathrm{~cm}^{2}$.

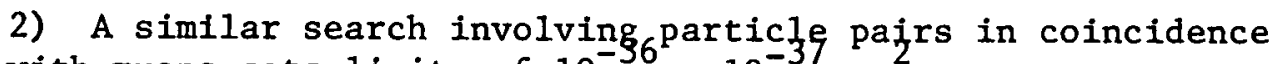
with muons sets limits of $10^{-36^{2}}-10^{-37} \mathrm{~cm}^{2}$.

3) The cross sections for all hadron pair combinations produced near $90^{\circ}$ by a $28.6 \mathrm{GeV}$ proton beam vary with invariant mass as $\mathrm{e}^{-5 \mathrm{~m}}$. The pair combinations fa $\neq_{1}$ into three groups: $\pi^{-} \mathrm{p} ; \pi^{+} \pi^{-}$; $\overline{\mathrm{p}} \mathrm{p}, \mathrm{K} \overline{\mathrm{p}}, \mathrm{K}^{+} \pi^{-} ; \mathrm{K}^{+} \overline{\mathrm{p}} ; \pi^{+} \overline{\mathrm{p}}, \mathrm{K}^{+} \pi^{-}, \mathrm{K}^{F_{\mathrm{K}}^{-}}$. Within each group, the yields coincide within 20 percent but among groups they differ by an order of magnitude. This behavior holds even for invariant masses of $5 \mathrm{GeV}$.

4) Results on inclusive simple hadron spectra and on short-range correlations of like-charge pion pairs are being evaluated.

Experiment ISR 804 has required design and development of large and economical drift chambers. Data acquisition will start during the fall of 1976 . 
UNIVERSITY OF MICHIGAN

Ann Arbor, Michigan

Contract No. EY-76-C-02-1112

Title of Project: Experimental High Energy Physics

Person in Charge: B. Roe, J. Vander Velde

Scientific and Professional Staff: D. Sinclair, C. Coffin, J. Chapman,

A. Seidl, R. Diamond, R. Ross

Scope of Work

This group is studying experimental high energy physics. The major effort currently is an investigation of interactions of neutrinos with matter using bubble chamber techniques. The group has historically intensively used bubble chamber techniques for the study of both weak and strong interactions. (The bubble chamber was invented at Michigan). They have built several bubble chambers and beams. Other experimental techniques involving visual devices or large amounts of data reduction have been utilized and are planned for the future.

The present effort involves two experiments, both utilizing the Fermilab 15' bubble chamber. The first is a study of neutrino interactions in hydrogen, a Michigan-Fermilab-California-Hawaii collaboration. The second experiment is a study of antineutrino interactions in a neon-hydrogen mixture, a Michigan-Fermilab-ITEPIHEP collaboration. (The latter two institutions are USSR laboratories). A 300,000 picture run in hydrogen and a 450,000 picture run in neon-hydrogen is scheduled. Currently about one half of the hydrogen run and about one ninth of the neon-hydrogen run is completed.

They are also finishing the latest of a series of pp and pd investigations at Fermilab, using the 30" MURA bubble chamber.

Goals and/or Major Results to Date

vp Experiment - It was established that hadronic jets produced in neutrino interactions have properties very similar to those produced in strong or electromagnetic interactions. The momentum of particles leaving the jet seems to be limited and has an average value independent of the jet mass or of the momentum transfer of the initial collision. This is valid to the highest elementary particle momentum transfer ever measured, $\left(Q^{2} \sim 50(\mathrm{GeV} / \mathrm{c})^{2}\right)$. One pion production has been measured and extends to broader values of momentum transfer than current theory predicted. Limits on 
UNIVERSITY OF MICHIGAN

Ann Arbor, Michigan

production of exotic resonances have been set. Deep inelastic scaling and inclusive distributions have been obtained.

$\bar{v} \mathrm{NeH}$ - Limits on dilepton production have been set, the antiquark content of the nucleon determined, and $n-p$ cross section ratios obtained.

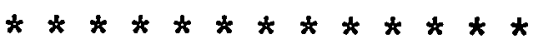

Title of Project: Spark Chamber

Person in Charge: D. Meyer

Scientific and Professional Staff: C. Akerlof, R. Thun. P. Alley

Scope of Work

The project involves the use of counter, drift chamber and other electronic techniques to study the properties of fundamental particles. In our most recent experiments the major innovation in technique has been the successful development and use of drift chambers. Coincident with this has been a shift from experiments related in theoretical interest to Regge trajectory type calculations to experiments which emphasize the nature of the quark constituents of hadrons. Our present experiment is a search for charmed particle production in hadronic reactions, a search made difficult by the large number of garden variety hadrons which one produced. For at least the next several years the efforts of the project will be on the systematics of these quark constituents.

Goals and/or Major Results to Date

Our most recent major result has been the systematic study of hadron-proton elastic scattering from 50 to $200 \mathrm{GeV}$ over an extended region in four momentum transfer.

Our present goal is to detect and measure the cross section for production of charmed particles in hadron-hadron collisions at $400 \mathrm{GeV}$ at Fermilab.

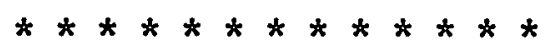

Title of Project: Particle Physics Theory and Phenomenology

Person in Charge: G. Kane, M. Einhorn

Scientific and Professional Staff: R. Cahn, J. Luthe, A. Arneodo 


\section{UNIVERSITY OF MICHIGAN \\ Ann Arbor, Michigan}

\section{Scope of Work}

Our activity in particle physics is broad based, covering most areas of current activity. Its underlying approach is largely to emphasize the important interplay between theory and experiment in solving fundamental problems. Thus on the one hand we might work with new theoretical ideas (our own or others) to see their consequences for experiment. Alternatively we might examine in detail the consequences of new experimental results for theoretical ideas."

\section{Goals and/or Major Results to Date}

Recently and in the near future some areas of concentration are as follows. Several areas of neutrino reactions, both exclusive and inclusive, are being studied. Interpretations of second class currents and ways to study them are being considered. Solvable field theory models are being considered and examined to see whether quark-model-like predictions occur and how important phenomena arise in detail. An approach to hadron two body reactions has been carried out in detail and a number of successful predictions for polarizations are being tested.

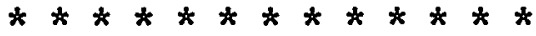

Title of Project: Particle Interactions and Field Theories

Person in Charge: Y. Tomozawa

Scientific and Professional Staff: Y. Yao, M. Chen, M. Machacek, U. Bar-Gadda

Scope of Work

The emphasis of our research is on the analysis of elementary particle interactions and their regularities based on a combined use of field theoretical tools and phenomenological approaches. This is an extremely effective approach which led us to the many fruitful developments in theoretical high energy physics in the past quarter century (quantum electrodynamics, internal symmetries. of particle interactions, current algebra and unified gauge theory of weak-electromagnetic and strong interactions, etc.).

The research activity of the group has been rigorous in the past years and many substantial results have been obtained. The subject matters range from the structure of non-abelian gauge 


\section{UNIVERSITY OF MICHIGAN \\ Ann Arbor, Michigan}

theories for the strong interactions as well as for the electromagnetic and weak interactions, the high energy behavior and the infrared problem, the analysis of the renormalization group equations, application of the Melosh transformation technique to non-leptonic weak interactions. Active studies of the recently discovered new resonances in the $e^{+}-e^{-}$collision have been carried out extensively.

Goals and/or Major Results to Date

The major results of our research activity to date include:

1) The Bloch-Nordsieck type cancellation of the infrared singularities in non-abelian gauge theories;

2) Successful application of the Melosh transformation method to non-leptonic weak decay processes;

3) The derivation of a set of homogeneous renormalization group equations based on the inhomogeneous Callan-Symanzik equations;

4) A proposal of the confinement potentials of quarks that are weaker than a linear potential; and

5) The numerical calculation of the g-2 factor for the electron to order $\alpha^{3}$.

The directions of research will be the study of the infrared structure of the non-abelian gauge field theories, the construction of a consistent model of the electromagnetic and weak interactions and a more extensive analysis of the new resonance spectroscopy.

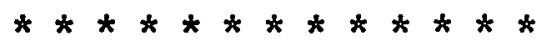

Ttrle of Prnject: Counter Group

Person in Charge: A. Krisch

Scientific and Professional Staff: K. Terwilliger, B. Sandler, K. Abe, R. Fernow, T. Mulera

Scope of Work

We have been studying $p-p$ elastic scattering at large $P_{\perp}^{2}$ in pure spin states using the ZGS polarized beam and our $\mathrm{He}^{3}$-cooled polarized proton target. We have also aided the ZGS staff in accelerating polarized protons to $11.75 \mathrm{GeV} / \mathrm{c}$ and have participated in accelerator research experiments on depolarization along with CERN and ANL. 


\section{UNIVERSITY OF MICHIGAN \\ Ann Arbor, Michigan}

The PPT V polarized proton target works reliably, but the high polarized beam intensity necessary to study high $P_{\perp}$ cross sections, causes radiation damage to the PPT. We are developing better ways to monitor and to remove the resulting loss of polarization.

We have been measuring 3-spin elastic cross sections by measuring the spin of the recoil proton with a carbon target recoll polarimeter. This requires hodoscopes and a CAMAC-computer readout system to remove asymmetries and biases, and was calibrated in an accelerated polarized beam to assure $4 \%$ precision. We are improving the polarimeter's range and precision.

The increased polarized beam intensity (now about $2 \times 10^{10}$ ) will greatly aid these experiments.

Goals and/or Major Results to Date

We measured $p-p$ elastic scaftering in pure initial spin states at $11.75 \mathrm{GeV} / \mathrm{c}$ in the range $P_{\perp}{ }^{2}=0.6 \rightarrow 2.2(\mathrm{GeV} / \mathrm{c})^{2}$. We found that both the spin-orbit forces (A) and the spin-spin forces $\left(\mathrm{C}_{n n}\right)$ decrease with energy in the smal1- $\mathrm{P}_{\perp}^{2}$ diffraction peak. Bût in the large $\mathrm{P}_{\perp}{ }^{2}$ region we find that $A$ is approximately independent of incident energy and $C_{n n}$ seems to grow in going from 6 to 11.75 $\mathrm{GeV} / \mathrm{c}$.

We measured 3-spin p-p elastic scattering at $6 \mathrm{GeV} / \mathrm{c}$ in the range $\mathrm{P}_{\perp}{ }^{2}=0.6 \rightarrow 1.0(\mathrm{GeV} / \mathrm{C})^{2}$; We find that $\mathrm{P}$ and $\mathrm{T}$ invariance are both obeyed to $\pm 4 \%$ at all $\mathrm{P}_{\perp}^{2}$ values. We also find that the double spin-flip cross sections are non-zero and in fact $10 \%$ as large as the non-filip.

We plan to study these spin effects at exactly $\theta_{\mathrm{cm}}=90^{\circ}$ where the Pauli exclusion principle is very important.

* * * * * * * * * * $*$ *

Title of Project: Search for New Effects in Nuclear Interactions at 500 Times Accelerator Energies

Person in Charge: $w$. Hazen

Scientific and Professional Staff: A. Hendel

Scope of Work

Only cosmic rays can be used to study particle interactions at lab energles $>1500 \mathrm{GeV}$ at the present time. We have completed 


\section{UNIVERSITY OF MICHIGAN \\ Ann Arbor, Michigan}

searchs for e/3 quark production and tachyon production at cosmic ray energies, and preliminary observations of high $p_{t}$ in interactions at cosmic ray energies. Now, we are completing a $35 \mathrm{~m}^{2}$ spark chamber array (1) to better define the production angle in a continuing search for massive e/3 quarks (produced at small angles), and (2) to study the high $p_{t}$ cross section at cosmic ray energles.

Goals and/or Major Results to Date

We expect to begin collecting data this fall. About one year will be required to obtain enough data for the statistical accuracy we desire. 
OAK RIDGE NATIONAL LABORATORY

Oak Ridge, Tennessee

Contract No. W-7405-ENG-26

Title of Project: High Energy Accelerator Shielding Studies

Person in Charge: R. G. Alsmiller, Jr.

Scientific and Professional Staff: H. Bertini, T. Gabriel, R. Santoro

Scope of Work

The purpose of the high energy accelerator shielding studies program at $\bar{O} \bar{R} \mathrm{NL}$ is to supply researchers with state-of-the-art calculated data in the area of radiation transport and to assist them, if desired, in developing their own calculational capabilities. The calculated data and researcher assistance cover the areasi of shielding, experimental setup, data interpretation, and instrumentation design (calorimeters). of particular interest at present are calorimeters. These devices, used to determine the energy and direction of incident particles, are being employed in many high energy experiments currently underway at BNL, FNAL, SLAC, and CERN, and ORNL has assisted and is continuing to assist many experimental groups in both the U.S. and Europe in the design of these instruments. Currently under investigation are calorimeters which employ $238_{U}$ as part of the device. This type of calorimeter appears very promising since part of the energy lost because of nuclear binding can be returned to the system due to the fissioning and capture characteristics of ${ }^{238} \mathrm{U}$.

Goals and/or Major Results to Date

1) To supply as requested by high energy hysics researchers stateof-the-art calculated data in the areas of shielding, experimental setup, data interpretation, and instrumentation design (calorimeters).

2) To maintain close communication with investigators so that important feedback information $c$ an be incorporated into future calculations.

3) To document and distribute data and calculational techniques for ready availability to other researchers.

4) To continually update, refine, and extend calculational procedures in the area of radiation transport. Many of the calorimeter design calculations have been compared with experimental calorimeter measurements, and good agreement between the calculated and experimental results has been obtained. 
OAK RIDGE NATIONAL LABORATORY

Oak Ridge, Tennessee

Contract No. W-7405-ENG-26

Title of Project: High Energy Physics

Person in Charge: $\mathrm{H}$. Cohn

Scientific and Professional Staff: H. Cohn, R. McCulloch, W. Bugg,

G. Condo, E. Hart

Scope of Work

High energy physics experiments are being analyzed to study the reaction mechanism and properties of elementary particles. Presently. we are involved in several large experiments in collaboration with other groups,' they include the study of the interaction of $8 \mathrm{GeV} / \mathrm{cm}^{-}$ with hydrogen, $15 \mathrm{GeV} / \mathrm{c} \pi^{+}$with deuterium, $\bar{p}$ at rest with deuterium, .. and $147 \mathrm{GeV} / \mathrm{cm}^{+}$and $\pi^{-}$with hydrogen. The latter experiment utilize a hybrid detector between a bubble chamber and position sensitive. counters resulting in data of superior accuracy to that obtainable by either tool used separately.

Goals and/or Major Results to Date

We were able to separate the two-prong inelastic from elastic events for $147 \mathrm{GeV} / \mathrm{c}^{-} \mathrm{p}$ interactions and conclude that the inelastic events. are predominantly diffractive dissociations of the beam or target particle. Other results from this experiment include the measurement of topological and leading particle cross sections, study of average charge multiplicity, examination of charged cluster emission, study of inclusive $\rho^{\circ}$ production, and examination of some exclusive channels. Preliminary results from the $8 \mathrm{GeV} / \mathrm{cm}^{-} \mathrm{p}$ bubble chamber experiment show evidence for structure in the $\mathrm{S}$ region (1920 MeV). Results from $\pi^{+} \mathrm{d}$ at $15 \mathrm{GeV} / \mathrm{c}$ confirm previous findings and a longitudinal phase space analysis of the reaction $\pi^{+} \mathrm{d} \rightarrow \pi^{+} \pi^{-} \mathrm{pp}_{s}$ is being done. The $\overline{\mathrm{p} d}$ experiment will be analyzed to shed light on the existence of bound or nearly-bound $\mathrm{NN}$ states. Our goals are to complete the analysis of these experiments and those which have been proposed and approved: We are also preparing new proposals for future runs, including a study of backward charge exchange processes.

$* * * * * * * * * * * * * *$ 
UNIVERSITY OF PENNSYLVANIA

Philadelphia, Pennsylvania

Contract No. $\quad$ EY-76-C-02-3071

Title of Project: Experimental and Theoretical High Energy

Physics Research

Person in Charge: A. Mann

Scientific and Professional Staff: E. Beier, S. Bludman, H: Brody,

L. Chang, S. Franke1, A. Klein, K. Lande, P. Langacker, T. Ling,

A. Mann, R. Morse, L. Resvanis, G. Segre, W. Selove, R. Steinberg,

J. Vergados, W. Wales, H. Williams; P. Cooper, L. Cormel1, M. Dris,

A. Entenberg, F. Krejs, C. Marshall, R. Patton, B. Robinson; W.

Cheung, J. Horstkotte, C. Li, M. Merlin, K. Raychaudhuri, H.

Tokeda, M. Vassanji; J, Cook, I. Fcrgusuili, W. Frati, C. Henry,

W. Kononenko, R. Marsha11, W. Mueller, R. Pearce, W. Schriver,

L. Shapiro, L. Taylor, R. Thern, R. Van Berg, R. Warburton, B.

Wilsker, B. Yost.

Scope of Work

There are five main facets of the high energy physics program at the University of Pennsylvania.

In strong interactions, we have two major experiments in progress at f'ermilab. One involves the study of single particle inclusive hadron-hadron differential and integral cross sections for a variety of incident and outgoing hadrons at many energies. This is part of a continuing Pennsylvania effort started in 1973 at Brookhaven National Laboratory to search for regularities in hadronhadron interactions. The second Fermilab experiment is an attempt to study quark-quark scattering through observation of hadron jets in the final states of hadron-hadron collisions. This experiment involves the development of new techniques in ionization calorimetry to make recognition of the hadron jets possible.

In weak interactions, our primary thrust is in neutrino physics. We have one major experiment in progress at Fermilab and another at Brookhaven. The experiment at Fermilab has been directed toward studiee of the deep inclastic interactions of high energy neutrinos and antineutrinos with matter. At Brookhaven the principal aim of the experiment is to clarify the space-time and isospin structure of the weak neutral current through measurements of the fundamental neutral current processes $\psi_{\mu}+p \rightarrow \nu_{\mu}+p$ and $\bar{\gamma}_{\mu}+p \rightarrow \bar{\nu}_{\mu}+p$, and weak neutral current single pion production. 
UNIVERSITY OF PENNSYLVANIA

Philadelphia, Pennsylvania

In the areas of combined weak and electromagnetic interactions, we have an experiment at the electron-positron storage ring (SPEAR) at the Stanford Linear Accelerator Center, the principal aims of which are to search for new particle production through observation of single muons and muon pairs, and to search for an angular asymmetry in muon pair production resulting from an interference between weak and electromagnetic amplitudes. We have also participated in experiments to test quantum electrodynamics at SPEAR.

In neutrino astrophysics, we have in progress an experiment, located in mines in Ohio and South Dakota and in the Mont Blanc tunne1, to. search for short-lived, strong sources of galactic neutrinos, in particular, neutrino bursts from dying supernovae.

Finally, there is a program in theoretical particle physics to interpret the new results of experiments in particle physics, which involves the phenomenology of new particle production by neutrinos, quark-lepton models of the fundamental particles and second class currents in gauge theories. Theoretical work in astrophysics and nuclear physics is also carried out.

Goals and/or Major Results to Date

The recent major results of this program are:

1) Measurement of the energy dependence of single particle hadron-hadron invariant cross sections which confirm predictions based on Pomeron factorization concerning the asymptotic behavior of hadron cross sections.

2) Joint participation in the discovery of weak neutral currents and determination of the parity-violating nature of the weak hadronic neutral current through the study of inelastic neutrino and antineutrino interactions.

3) The discovery of neutrino and antineutrino induced dimuon events which have been taken as the first explicit evidence for the existence of hadronic matter carrying a new quantum number, generally referred to as. "charm."

4) First measurements of the differential cross sections for the basic processes: $\nu_{\mu}+p \rightarrow \nu_{\mu}+p$ and $\bar{\nu}_{\mu}+p \rightarrow \bar{\psi}_{\mu}+p$.

$$
* * * * * * * * * * * * * * *
$$


PRINCETON UNIVERSITY

Princeton, New Jersey

Contract No. EY-76-C-02-3072

Title of Project: High Energy Physics Group

Person in Charge: F. Shoemaker

Scientific and Professional Staff: F. Shoemaker, V. Fitch, P. Piroue,

A. Smith, D. Jensen, K. McDonald, G. Sanders, P. Surko, J. Thaler,

R. Webb, M. Withere11; R. Cester-Regge, R. Sumner, L. Kluberg; J .

Rranson, R. Kadcl, J. Muelle1, J. Whictaker.

Scope of Work

This project supports research in elementary particle physics by the Facully of the Department of Physics of Princeton University, their Graduate Students, and Research Associates. The work is primarily experimental, although there is some theoretical work supported by the contract. The experimental research has, in recent years, been entirely carried out at the Brookhaven National Laboratory and the Fermi National Accelerator Laboratory. The apparatus for the experiments is constructed at Princeton, moved to the accelerator laboratory for setup and data acquisition, and the data is subsequently analyzed with the use of computers, both at Princeton and at the National Laboratories. For some experiments, other institutions collaborate with Princeton supplying a part of the staff and apparatus for the experiment.

At present, one experiment at BN. is searching for charmed mesulus produced in proton-antiproton annihilations, and sluultaneously conducting a sensitive test of $\mathrm{C}$ invariance in strong interactions. Another group, in collaboration with the University of Massachusetts, is studying the beta decay of the lambda hyperon. At FNAL, a collaboration with the University of Chicago has been studying particle production at large transverse momenta in a series of experiments. Another FNAL experiment, a collaboration with another group at the University of Chicago, is studying muon pairs produced by hadrons. An experiment being set up at FNAL, in collaboration with the University of California, Berkeley, will study rare muon induced processes.

Goals and/or Major Results to Date

Within the last decade, the outstanding results from our project include:

1) The discovery of $C P$ non-invariance in the decay of the $K_{L}^{0}$.

2) The measurement of the $K_{L}^{O} \rightarrow \pi^{\circ} \pi^{\circ}$ branching ratio. 


\section{PRINCETON UNIVERSITY}

Princeton, New Jersey

3) The discovery of hadron production at exceptional transverse momenta, and the direct production of leptons in excess of those from meson decay.

4) A definitive study of CP-violating weak decays.

5) A complete measurement of the muon pair spectrum from hadron interactions which has shown that pairs account for all of the. direct muon production.

$$
* * * * * * * * * * * * * *
$$


UNIVERSITY OF ROCHESTER

Rochester, New York

Contract No. EY-76-C-02-3065

Title of Project: High Energy Physics General Research/Development (Experimenta1)

Person in Charge: A. Melissinos

Scientific and Professional Staff: A. Bodek, T. Ferbel, A.'Melissinos,

S. Olsen, P. Slattery, W. Metcalf, D. Gross, W. Mollet, T. Oshima,

T. Jensen, D. Chaney, B. Kern, M. Millcr, D. McCal, J. LeBritton,

C. Chandlee, D. Nitz, J. Biel.

$\underline{\text { Scope of Work }}$

The scope of the Particle Physics program is to carry out original and basic research on the properties and interactions of the Elementary Particles. Our research groups conduct experiments using mainly the facilities of, the Fermi National Accelerator Laboratory at Batavia, Illinois, and of Brookhaven National Laboratory on Long Island, N.Y. Much of the detection equipment is designed and constructed at Rochester, where we also perform a fraction of the computer analysis of the experiments.

The activities of the group during the past year can be classified in approximately six distinct areas:

1) Investigation of muon interactions performed at BNL.

2) Etudy uf Coulomb and dittractive dissociation of neutrons, and charm-particle searches at Fermilab.

3) Preparation of experiment to study charged-particle dissociations at Fermilab.

4) The diffraction scattering program at the internal target area of Fermilab.

5) Neutrino interactions and short-lived particle search at Fermilab.

6) Phélumcnological investigations of bubble chamber data from Fermilab.

The program we have just sketched out is an unusually broad one for a group of our size. However, our research activities are focuscd on important problems in particle physics, and the effective utili-. zation of our resources and especially the multiple use of installed 
UNIVERSTTY OF ROCHESTER

Rochester, New York

experiments, has.enabled us to contribute continually to the development of the field of high energy physics.

Goals and/or Major Results to Date

The goals and major results of the activities itemized under scope follow below:

1) Data have been taken at BNL on inelastic muon scattering at small values of the Bjorken $\mathrm{X}$-variable. In addition, measurements have been made of trident production and of the A-dependence of $\mu$-nuclear scattering. Presently the apparatus is being modified for studies of dimuons using an incident high-flux $\pi^{-}$beam.

2) Our investigation of the dissociation of neutrons into ( $\mathrm{Pf}^{-}$) systems has produced several surprises:

a) The data require large contributions from baryonexchange in addition to the usual T-exchange Deck process.

b) New structure has been found in the t-distribution of produced ( $\mathrm{p} \mathrm{fH}^{-}$) systems.

c) A dramatic correlation exists between the decay properties and production t-distribution of the dissociated system and its mass.

New data taken to search for charm-particle production are being analyzed. A prompt muon trigger was utilized in the experiment to improve the charm signal/noise ratio.

3) Coulomb and diffractive excitation of $\pi^{-}, \mathrm{K}^{-}$, and $\overline{\mathrm{p}}$ is expected to be carried out in the summer of 1977. A drift chamber:system has been designed and is being constructed.

4) Data have been obtained for the inclusive reactions pd $\rightarrow d+$ anything and $p p \rightarrow p+$ anything. Analysis is in progress to extract triple Regge parameters from the measurements. In addition, a large-angle spectrometer has been set into operation, which, in conjunction with a room-temperature gas jet target, was used recently to obtain data on large-angle elastic scattering.

5) The apparatus for the detailed investigation of $\mu^{+} \mu-$ production in $\nu$-nuclear interactions is being constructed. In addition, preliminary studies of the production of single and dimuon events in hadron-nuclear collisions at Lab $E$ are in progress. 


\section{UNIVERSITY OF ROCHESTER \\ Rochester, New York}

6) Correlations between the transverse momentum and the mass of a system produced in pp collisions at $400 \mathrm{GeV} / \mathrm{c}$ are revealing unusual features of hadroproduction. The transverse momentum of a bundle of hadrons appears to be essentially independent of the charge or multiplicity of the system, but is rather a function of its mass alone. In addition, new fits to inelastic multiplicities in hadron-proton collisions have provided direct evidence for Pomeranchuk factorization at high energies.

$$
* * * * * * * * * * * * * *
$$

Title of Project: High Energy Theory

Person 1n Charge: S. Okubo

Sclentific and Professional Staff: S. Okubo, C, Hagen, V. Mathur

Scope of Work

The scope of the Theoretical High Energy Physics Program at Rochester consists of the investigation and application of the basic laws and principles governing elementary particle interactions. Our main activity is most conveniently described within the framework of three somewhat distinct sub-fields. The first of these consists of an ongoing investigation of the symetry principles which underlie the basic weak, electromagnetic, and strong interactions. At the moment, we are studylng normal hadronic decay modes of the charmonium $\Psi$ and $\Psi^{\prime}$, various consequences of the quark-1ine rule, and weak decay widths of the recently discovered charmed bosons. Since the minimum symmetry group governing the new physics appears to be the $\mathrm{SU}(4)$, we are also interested in studies of the general $S U(n)$ group as well as the more general sem1-simple Lie algebra.

The second main area of activity in our group is that of Lagrangian field theory. Recent work here includes the topics of the magnetic monopole, quark-confinement in two dimensional space-time, and 1ight-cone quantization. The last of jur acrivities is racher miscellaneous. We are interested in obtaining rigorous bounds of various physical quantities such as $K_{\ell 3}$ decay parameter on the basis of the analiticity and unitarity. Also, phenomenological investigations of high energy scattering processes have been undertaken from time to time. 


\section{UNIVERSITY OF ROCHESTER \\ Rochester, New York}

Goals and/or Major Results to Date

Our goal is to unravel the basic laws governing the elementary. particle interactions. Our major results on the symmetry principle to date are derivation of the SU(3) mass formula, nonet scheme for the vector mesons, calculations of $\pi^{+}-\pi^{0}$ mass difference, studies of the chiral SU(3) symmetry, and recently some work on the charmoniums. Also, rigorous mathematical formulation of various dispersion inequalities has been obtained with many applications. With respect to field theory, work here on high-spin particles, and magnetic monopole are of significant importance.

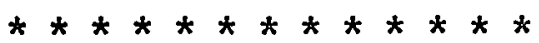


UNIVERSITY OF WISCONSIN

Madison, Wisconsin

Contract No.

$\mathrm{EY}-76-\mathrm{C}-02-0881$

Title of Project: High Energy Physics - Experimental

Person in Charge: D. Reeder, S. Aronson, U. Camerini, D. Cline, A. Erwin, W. Fry, R. March, L. Londrom, W. Prepost, M. Thompson

Scientific and Professional Staff: A. Benvenuti, G. Bunce, R. Handler, E. Harvey, Johnson, J. Learned, R. Loveless, J. Mapp, T. Rhoades, M. Sheaff, C. Spencer, M. Thompson, J. Von Krogh

Scope of Work

The experimental investigations performed under this contract are designed to discover and define in detail the characteristics and interactions of elementary particles. The techniques employed range from bubble chambers to proportional wire chambers, from conventional spectrometers to massive neutrino detectors and even to the design and construction of special accelerators.

These experiments are performed with high energy protons incident on fixed targets, colliding beams of electrons or hadrons, and cosmic rays.

An area of concentrated effort is the interaction of high energy neutrinos studied both using counters and using bubble chambers. Neutrino interactions are particularly suited to a search for new types of hadronic quantum numbers and to the spectroscopy of leptons and hadrons. Moreover, the point like nature of the weak current enables the neutrino "microscope" to illuminate the substructure of hadronic matter and to study the dynamics of these constituents. These investigations are also pursued where appropriate at electron accelerators and $\mathrm{e}^{+} \mathrm{e}^{-}$colliding beams.

The interactions of hadrons, particularly those characterized by strangeness, is an excellent laboratory to test constituent models. The efforts to obtain storage rings of antiprotons with which to collide with protons at high energy are very promising and would permit the testing of gauge theories by searching for the characteristic heavy bosone.

Goals and/or Major Results to Date

The program has had many recent successes. Among them are:

1) First observation of dimuons directly produced by neutrinos. This result implies a new hadron with a new quantum number and which decays by weak interaction. 
UNIVERSITY OF. WISCONSIN

Madison, Wisconsin

2) First observation of $\mu$ e dilepton events produced by neutrinos in a bubble chamber. This result linked the dilepton production with strange particles as is predicted by "charm" theories.

3) First observation of antineutrino proton elastic scattering and confirmation of $\nu$ proton elastic scattering. This result is the first concerning the most simple and fundamental reaction produced by the weak neutral current and has important implications for theories of the neutral current.

4) Observation of effective parity violation in the weak neutra1 current at high energies. This result adds additional support for gauge theories.

5) Observation of spin polarization of Lambda hyperon's produced inclusively by nuclear targets at hịg energy.

$$
* * * * * \dot{*} * * * * * * *
$$

Title of Project: High Energy Physics - Phenomenology

Pesson in Charge: V. Barger

Scientific and Professional Staff: D. Cline, F. Halzen, M. Olsson,

D. Reeder, Molodowich, Nanopoulos, M. Singer

\section{Scope of Work}

The current research activities of the phenomenology group include the following subjects:

1) Deep inelastic neutrino scattering;

2) Current fragmentation models of new particle production;

3) Weak decays of new particles;

4) Consequences of right-handed weak currents;

5) Dilepton events produced by neutrinos;

6) Neutral currents and new quark models;

7) Diumons from inelastic $\pi \mathbb{N}$ scattering; 


\section{UNIVERSITY OF WISCONSIN}

Madison, Wisconsin

8) Intermediate vector boson production by $\mathrm{pp}$ and $\overline{\mathrm{p}} \mathrm{p}$ collisions;

9) Optical models for polarized pp cross sections;

10) Analyses of current algebra predictions of low energy mesonnucleon scattering, photoproduction, and neutrino production;

11) Estimates of charmed particle production cross sections in hadronic collisions;

12) Jet production at high transverse momentum in inelas tic collisions; and

13) Zweig-rule breaking in $\Psi$-decays.

A major thrust of our program is the phenomenological analysis of data and theoretical models for charm particle production. A substantial fraction of this research effort is in the weak and electromagnetic interaction areas. In addition we are maintaining an active research program in strong interactions. A significant part of our research program is closely allied with the current Wisconsin experimental research program.

\section{Goals and/or Major Results to Date}

The basic goals of our phenomenology program are to confront theorists of particle interactions with the experimental data, to formulate new theoretical models, and to search for empirical regularities in the data which could lead to new theories. Major results to date include:

1) A comprehensive analysis of the role of right-handed currents in deep-inelastic neutrino scattering; b-quark production is necessary to account for $\left\langle y>i N\right.$ and $\sigma_{t}\left(U_{N}\right) / \sigma_{t}(\mathrm{~N})$ data;

2) An investigation of the role of new quarks in neutral current processes: The Weinberg-Salam and b-quark models are not distinguished by present data; and

3) Predictions for $W$-boson production at proposed colliding ring machines.

$$
* * * * * * * * * * * * * *
$$


UNIVERSITY OF WISCONSIN

Madison, Wisconsin

Title of Project: High Energy Physics - Theory

Person in Charge: W. Kraushaar

Scientific and Professional Staff: V. Barger, L. Durand, M. Ebel,

C. Goebe1, F. Halzen, M. O1sson, M. Singer, B. Durand

\section{Scope of Work}

This is a general program on the theory of fundamental particles and the interactions between them, with emphasis on the theoretical description and interpretation of experimental results in this field. Problems related to strong, electromagnetic, weak, and gravitational interactions are under investigation. Specific problems of interest at the present time include:

1) Spontaneously broken gauge theories; an investigation of topologically interesting models of baryons.

2) Magnetic monopoles; construction of a formalism which describes the interaction of an extended monopole with a charged particle.

3) Weak decays of new particles; calculations of decays from a phenomenological Lagrangian for the hadronic currents.

4) Lie theory and addition formulas for Jacobi functions of the second kind; demonstration of a connection of addition formulas of the representation of Lie groups by integral transformations.

5) e- $\mu$ mass ratio; consequences of various proposed lepton multiplets for the gauge theory calculation of the $e-\mu$ mass ratio.

6) Distributions for weak semlleptonic decays of new particles; consideration of form factor effects and multiparticle decay modes.

7) Vector mesons and direct leptons: The existence and properties of old and new vector mesons as we 11 as the $W$ mesons are related to the observed production direct leptons.

8) Current Algebra hudels of scattering processes: Current algebra and PCAC models for hadronic scattering processes, electroproduction and neutrino production. 


\section{UNIVERSITY OF WISCONSIN \\ Madison, Wisconsin}

\section{Goals and/or Major Results to Date}

The goals of our theory program are to investigate the properties and interactions of the elementary particles. Major results to date include:

1) The resolution of an ambiguity in models for pion production from nucleons near threshold and the relation to pion-pion scattering.

2) Calculation of photoproduction multipoles and the $(3,3)$ phase shift using a current algebra. model.

3) Usc of neutrinns to probe gauge theories.

4) Vector dominance and the radiative decays of new and old mesons.

5) S-matrix treatment of many overlapping resonances.

6) Probing the new particles with hadron beams.

7) Barrier top resonances and heavy ion reactions.

$$
* * * * * * * * * * * * *
$$


YALE UNIVERSITY

New Haven,. Connecticut

Contract No. $\quad$ EY-76-C-02-3075

Title: of Project: Theoretical Research on Elementary Particles and Interactions

Person in Charge: F. Gursey, S. MacDowe11, C. Sommerfield

Scientific and Professional Staff: F. Gursey, S. MacDowe11; C. Sommerfield, T. Appelquist, I. Bars, F. Mansour1, J. Healy

Scoep of Work

Three major topics covered by our group are:

1) Gauge Field Theory of Strong Interactions (chromodynamics) and Unified Field.Theories of Strong, Electromagnetic and Weak Interactions;

2) Quark Confinement Problems and Models for Confined Quarks; and

3) Geometrization of Gauge Theories based on Lie Algebras and Graded Lie Algebras.

Goals and/or Major Results to Date

1a) T. Appelquist et. al. have demonstrated the Infrared finiteness of chromodynamics in perturbation theory. Discoveries of new particles appear to be consistent with the original charmonium model. The spectrum and decay of new particles (including channed hadrons) will be investigated within chromodynamics.

1b) F. Gursey, P. Ramond, and P. Sikivie have shown the spontaneously broken $\mathrm{E}_{7}$ model to be phenomenologically viable. The detailed structure of the neutral current and the mass matrix are under investigation.

2a) C. Sommerfield et. al. have discovered an exact classical solution with finite energy. The goal is to find vortex-1ike solutions showing confinements.

2b) I. Bars has exhibited a remarkable correspondence between his new string theory and 2-dimensional chromodynamics. The goal is a generalization to 4 dimensions.

2c) Healy et. al. have developed a consistent scattering theory of bound quarks in Quantum Mechanics. A generalization to field theory is contemplated. 
YALE UNIVERSITY

New Haven, Connecticut

2d) A. Chodos' goal is the construction of a fermion lattice with three flavors as a model for a gauge theory of quarks in the strong coupling approximation.

3a) F. Mansouri et. al. have constructed a unified fiber bundle leading to a curvature that generalizes the combined Einstein and Yang-Mills Lagrangians.

3b) F. Mansouri and S. MacDowell are proposing generalized fiber bundles for the description of local supersymmetry for gauge theorles involving both fermions and bosons.

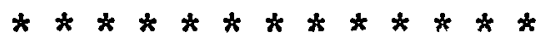

Title of Project: Yale Accelerator Users Group

Person in Charge: J. Sandweiss, R. Adair

Scientific and Professional Staff: R. Adair, H. Kasha, R. Kellogg,

H. Kraybill, D. Ljung, T. Ludlam, R. Majka, P. Martin, J. Marx,

P. Nemethy, J. Sandweiss, A. Slaughter, H. Taft

Scope of Work

Counter Experiment Projects: During the past two years, this group has made measurements of the properties of prompt muons produced in the collisions of high energy hadrons at Fermilab.

The Yale measurements demonstrated that a) the muons were produced in pairs; b) the muons were not polarized along their direction of flight; and c) that the flux of muons produced at large energies was considerably larger than could be accounted for by conventional processes. A set of experiments at Brookhaven National Laboratory has the goal of better defining the specific character of this production process. After these measurements are completed, they plan to investigate the possible existence of time-reversal invariant effects in the weak interaction decays of hadrons (specifically, the $\mathrm{K}$-mu-3 decays of the $\mathrm{K}_{\mathrm{L}}^{\mathrm{O}}-$ mesnn). They plan to teat the predictions of new unified theories by measuring the very small $(0.1 \%)$ polarization predicted by these unified field hypotheses.

The High Resolution Streamer Chamber Project: A high pressure (20-40 atmosphere), high resolution, etrcamcr chamber has been designed and is under construction at Yale. The pulsing system and optics are designed to realize the potential gain in streamer resolution afforded by the high pressure operation. The chamber 
YALE UNIVERSITY

New Haven, Connecticut

will be used in an experiment at FNAL to search for the production and decay of new short-lived particles with lifetimes in the range $10^{-14} \mathrm{sec}$ to $10^{-11} \mathrm{sec}$.

The Yale Bubble Chamber Effort: We are presently engaged in a series of experiments at the Fermi National Laboratory to investigate particle production via the strong interaction with meson and nucleon beams of $150 \mathrm{GeV} / \mathrm{c}$ momentum impinging on a hydrogen bubble chamber. A detailed study of particle distribution and correlations in momentum space should help to limit the choices for a specific theoretical framework within which to describe the nuclear force.

For these experiments the group has participated with several other universities in the construction of a "hybrid" spectrometer system at Fermilab in which the 30 " bubble chamber is augmented with external electronic detectors. A Cerenkov counter upstream of the bubble chamber tags each incident particle according to its mass, allowing the use of unseparated beams. An array of proportional wire planes downstream of the bubble chamber records the trajectories of the fast secondary particles. An array of lead glass Cerenkov detectors is used to measure high energy photons exiting the bubble chamber, and a system consisting of a gas Cerenkov counter plus a relativisticrise ionization sampling device measures the masses of charged secondaries. The currently approved experimental program calls for approximately 1.2 million exposures of this system to beams of $\pi$ mesons, $\mathrm{K}$ mesons, and protons.

The Yale group has developed: and operates a computer-controlled. automatic film measuring device (PEPR) for its bubble chamber work.

Goals and/or Major Results to Date

At this time one part of the extended group's seffort is devoted to further measurements of the properties of leptons produced directly in hadron interactions and will be preparing an experiment concerned with time-reversal invariance in elementary particle interactions. Another part of the group's personnel is involved with the design and construction of a new instrument which should be particularly valuable for investigations of short-lived hadronic particles such: as one expects from the newly discovered class of "charmed" particles. The third part of the group's effort is directed towards the use of bubble chamber techniques in the disentanglement of the complex structures of very. high energy hadron-hadron collisions.

$$
\text { *************** } * *
$$


YALE UNIVERSITY

New Haven, Connecticut

Contract No.

$\mathrm{EY}-76-\mathrm{C}-02-3075$

Title of Project: High Energy Elementary Particle Interactions

Person in Charge: V. Hughes, M. Zeller

Scientific and Professional Staff: W. Hughes, M. Zeller, M. Lube11, P. Souder, D. Lu, P. Schuler, M. Alguard, J. Clendenin, S. Dhawan, R. Fong-Tom

Scope of Work

Our group's primary activity is the study of polarization phenomena in high energy scattering. Experiments include depp inelastic scattering of polarized electrons by polarized protons at SLAC, high energy elastic scattering from polarized protons at FNAL, and both elastic and inelastic scattering of $\pi ; K$, and $P$ from polarized protons at BNL. In addition to the high energy experiments, our work includes the development of polarized particle beams (electrons and protons) and polarized protons and deuteron targets.

Goals and/or Major Results to Date

At SLAC our work led to the first high energy polarized electron beam. The deep inelastic scattering of polarized electrons by polarized protons provides the only information about the spindependent form factors of the proton and tests scaling, high energy sum rules, and specific models of proton structure. In addition, scattering of polarized electrons by unpolarized nucleons has led to a test of parity conservation relevant to the gauge theories of weak and eleatromagnetic interactions. At BNL our polarization studies have contributed particularly to the search for exotic $2 *$ particles, and more generally to the understanding of the $\mathrm{K}^{+} \mathrm{p}$, $\mathrm{K}^{-} \mathrm{p}$, and $\overline{\mathrm{P}} \mathrm{P}$ interactions. Initial results have just been obtained in the high energy elastic scattering from polarized protons at FNAL.

Further experiments are planned at SLAC with polarized electrons to study proton structure and to investigatc parity nonconservalion, at BNL to measure polarization effects in inelastic hadron interactions, and at FNAL to measure polarization phenomena at high energies.

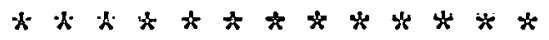


PART III

OTHER PROGRAMS 
ADELPHI UNIVERSITY

Garden City, New York

Contract No. EY-76-S-02-4064

Title of Project: Theoretical Studies and Analysis of High Multiplicity Gamma Ray Events in PP Collisions at ISR

Person in Charge: J. Dooher

Scientific and Professional Staff: J. Dooher

Scope of Work

Th1s project involves continuing studies on high multiplicity gamma ray events observed in high energy $p-p$ collisions. A comparative analysis will be performed with data from Brookhaven-Rome-Adelphi (BRA) ISR multigamma search.

The original BRA experiment was divided into two phases, an exploratory search for multigammas in collaboration with the CERN, Columbia, Rockefeller University group, and a detailed experiment with a capability of obtaining a large amount of related data. The purpose was to characterize high multiplicity gamma ray events in $p-p$ collisions. Besides a study of hadronically initiated events, a search for new processes such as magnetic monopole production and annihilation is being undertaken. The first phase has been completed and its results published. ${ }^{1}$ The second has been underway for a year, and the data taking essentially completed. The program will apply theoretical models developed during the initial research. These models are based on independent pion emission and independent cluster production.

Goals and/or Major Results to Date

The ongoing research has been motivated by attempts to understand the results of our exploratory experiments in terms of appropriate models of pion production, as well as the extent to which hypothetical gamma ray production processes such as magnetic monopole pair and high $Z$ lepton pair production and annihilation could contribute to the high multiplicity gamma ray tail. A new area of investigation which has arisen is the possibility of analyzing gamma ray cascades from new particle states relating to the $\Psi$ and $J$ particles.

The major effort in the project will be to compare the predictions of realistic models of multi-particle production, cluster emission, with our data on gamma ray multiplicity distributions. 


\section{ADELPHI UNIVERSITY}

Garden City, New York

1 Dell, G.F.; Uto, H.; Yuan, L.C.L.; Amaldi, E.; Beneventano, M. Borgia, B.; Pistilli, P.; Sestili, I.; Dooher, J.; "An Investigation of High-Multiplicity Gamma Events in pp Collisions with c.m. Energies between 22 and $62 \mathrm{GeV}$." Lettere A1 Nuovo Cimento, Vol. 15, n.8, Feb., 1976.

$$
* * * * * * * * * * * * *
$$




\section{BRANDEIS UNIVERSITY \\ Waltham, Massachusetts}

Contract No. EY-76-S-02-3230

Title of Project: Research in High Energy Physics

Person in Charge: H. Schnitzer

Scientific and Professional Staff: H. Schnitzer, J. Kang, L. Abbott, P. Townsend

\section{Scope of Wurk}

1) Development of non-perrurbaclve mitthods in quantum fifld theory, with particular emphasis on the $1 / \mathrm{N}$ expansion. The leading term of this approximation is the Hartree approximation.

2) The study of meson spectroscopy, with particular emphasis on the charmonium system. The main effort is presently to understand the spin-dependent corrections appearing in linear potential models of quark confinement.

Goals and/or Major Results to Date

1) We have shown that the $1 / \mathrm{N}$ expansion is a consistent approximation scheme for $\lambda \phi^{4}$ theory with $O(N)$ symmetry in four space-time dimensions.

2) A qualitative understanding of the spin-dependent structure of charmonium has been achieved.

$$
* * * * * * * * * * * * *
$$

Title of Project: Research in Elementary Particle Physics

Person in Charge: L. Kirsch

Scientific and Professional Staft: P. Schmldl, J. Benoinger,

S. Jacobs

Soope of Whrk.

The work of this group is focused on the study of elementary particle interactions using as a source the medium energy separated beam and high energy unseparated beam at Brookhaven National Laboratory and as a detector the Multiparticle Spectrometer. 
BRANDEIS UNIVERSITY

Waltham, Massachusetts

Goals and/or Major Results to Date

Recently completed experiments have provided data on the dynamics of antiproton annihilation into neutral strange particles and unequivocal evidence of a high mass $\mathrm{K}$-meson. New experiments will be sensitive searches for the associated production of "charmed" particles.

$* * * * * * * * * * * * *$ 
BROWN UNIVERSITY

Providence, Rhode Island

Contract No. EY-76-C-02-3130

Title of Project: A Research Program in Theoretical High Energy Physics

Person in Charge: D. Feldman

Scientific and Professional Staff: H. Fried, G. Guralnik, K. Kang, P. Westervelt, C. Tan, J. Kim

Scope of Work

During the coming year the major effort of the theoretical program will be devoted to studies in the physics of high energy phennmena, the properties of elementary particles, and quantum field theory. Particular problems to be considered will include the analysis of high energy reactions (analytic parametrization, coupling of the bare Pomeron, triple-Regge cross sections); mul.tiparticle processes (field-theoretic origin of triple-Pomeron coupling, eikonal and lattice approaches to Reggeon field theory); narrow-width particles (unified potential scheme, $\Psi$ - and "upsilon" particle); spectral sum rules (SU(4) and axial-vector meson mass formulas); weak neutral currents; bound-state-induced solutions (Yang-Mills theories, two-dimensional models, anharmonic oscillator, $\phi^{4}$ theory); quark confinement; quantum field-theoretic models in curved space-time. It is also planned to devote some time to other problems of interest and importance in the theory of elementary particles as they may arise, as well as to certain aspects of the general theory of relativity.

Goals and/or Major Results to Date

Last year's research program dealt with a variety of topics including multiparticle production models and hadron dynamics (Pomeron interaction in elkonal field theory, dual-Regge topological expansion and the bare Pomeron, unitarization of dual amplitudes); spectral sum rules and $\mathrm{SU}(4)$ (mixing angles, mass relations); $\mathrm{SU}(2) \times \mathrm{U}(1) \times \mathrm{U}(1)^{\prime}$ gauge model ( $\mathrm{K}^{0}-\overline{\mathrm{K}}^{0}$ mass difference, dimuon events); non-Feynman perturbative approaches to field theory (collective phennmena in $\phi^{4}$ theory, bound-state approximation to the Nambu-Jona-Lasinio mode1); topics in quantum statistical mechanics and general relativity; as well as other studies involving the strong, electromagnetic, and weak interactions of elementary particles.

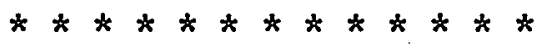




\section{BROWN UNIVERSITY \\ Providence, Rhode Island}

Title of Project: Electronic Detector-Spark Chamber Group and Bubble Chamber-Hybrid System Group

Person in Charge: R. Lanou, Jr., A. Shapiro

Scientific and Professional Staff: A. Shapiro, R. Lanou, Jr., M. Widgoff, J. Massimo, D. Peaslee, D. Cutts, D. Brick, R. Dulude

$\underline{\text { Scope of Work }}$

The experiments of the electronic detector-spark chamber group include:

1) A search for high mass boson resonances and determination of quantum numbers in antiproton-proton systems;

2) A nucleon-nucleon and meson-nucleon elastic and quasi-elastic scattering experiment at the highest energies;

3) A study of inclusive scattering, with associated multiplicity and pseudo-rapidity distributions, for hadrons on protons to hadrons at incident energies up to $175 \mathrm{GeV}$;

4) An experiment to study the A-dependence of hadronic inclusive scattering;

5) A search for charged, long-lived, massive particles up to the kinematic limit at Fermilab; and

6) A study of the hadronic final states produced by $100 \mathrm{GeV}$ pions in association with direct leptons.

The bubblc chamber-hybrid system group is primarily engaged in several experiments at the Fermilab. A study of $\pi^{-} p$ interactions at $147 \mathrm{GeV} / \mathrm{c}$ is belng completed, and a larger investigation of $\pi^{+} \mathrm{p}, \mathrm{K}^{+} \mathrm{p}, \mathrm{pP}$, and $\pi^{-} \mathrm{p}$ collisions at $147 \mathrm{GeV}$ has begun. To the proportional hybrid system has been added a prototype forward gamma detector, enabling the precision measurement of up to 6 photons $\left(3 \pi^{\circ} \mathrm{s}\right.$ and/or $\left.n^{\circ} \mathrm{s}\right)$. Major aspects of multiparticle production using four different projectiles are being pursued. A precision intercomparison of corresponding processes produced by the different projectiles is underway, using identical experimental conditions.

Goals and/or Major Results to Date

Electronic detector-spark chamber group: 
BROWN UNIVERSITY

Providence, Rhode Island

1) Cross sections for $\bar{p} p+\pi^{0} \pi^{0}, \pi^{0} n^{0}, n^{0} n^{0}$, and angular distributions for the former two in the mass range $2035-2500 \mathrm{GeV}$; and

2) Elastic cross sections and angular distributions for $\pi^{ \pm}, \mathrm{K}^{ \pm}, \mathrm{p}^{ \pm}$proton scattering at $50,70,100,140,175 \mathrm{GeV} / \mathrm{c}$.

Bubble chamber-hybrid system group:

1) Cross sections and charged multiplicity distributions for $\pi^{-} p$ and $\mathrm{K}^{-} \mathrm{p}$ interactions at $147 \mathrm{GeV} / \mathrm{c}$;

2) Inelastic two-prong events in $147 \mathrm{GeV} / \mathrm{c} \pi^{-} \mathrm{p}$ interactions;

3) Inclusive and semi-inclusive $\rho^{\circ}$ production at $147 \mathrm{GeV} / \mathrm{c}$; and

4) Evidence for charged cluster emission, and studies of the reaction $\pi^{-} \mathrm{p} \rightarrow \pi^{-}$fast $+\mathrm{X}$ at $147 \mathrm{GeV} / \mathrm{c}$.

$$
* * * * * * * * * * * * *
$$




\section{UNIVERSITY OF CALIFORNIA}

Davis, California

Contract No. EY-76-S-03-0034, 191

Title of Project: High Energy Particle Physics Research

Person in Charge: Richard L. Lander

Scientific and Professional Staff: J. Klems, W. Ko, D. Pellett,

P. Yager

\section{Scope of Work}

Experimental study of high energy interactions. Largely bubble chambers, but some streamer chamber and counter work also. Emphasis is on hadronic interactions. Fermilab energies dominate. Main thrust is on exploring those features that bear on general properties. Thus inclusive distributions, charm. searches, quantum statistical effect, and "Zweig rule" tests are emphasized rather than, say, meson spectroscopy. The FNAL 30-inch (with PWC) and 15-foot chambers dominate our present work. We have, in various stages of analysis, pp interactions at 100, 200, and $400 \mathrm{GeV}$; $\pi^{+} \mathrm{p}$ at 100 and $200 ; \pi^{-\mathrm{d}}$ at 200 and $360 \mathrm{GeV} ;$ and $\mathrm{K}^{+} \mathrm{p}$ at $11.8 \mathrm{GeV} / \mathrm{c}$.

Studies in progress are off-mass-shell $\pi$ and $\mathrm{K} \pi$ scattering, Mueller factorization, rescattering effects in $\pi^{-} \mathrm{d}$ interactions (space-time development picture of hadronic interactions), and the two-component model (diffractive plus Poisson).

Davis-LBL is one of 3 U.S. groups evaluating the 15 -foot BC for hadron physics. In our system, LBL computer-controlled "Franckensteins" (COBWEB) measure inward1y from track end-points toward the region of confusion (vertex). Image Plane Digitized scanning allows automatic positioning of Franckensteins. This system should be capable of measuring up to $\sim 50 \mathrm{~K}$ events per ycar. off-line correction of scanning errors (very time consuming) will be virtually eliminated by a UCD-built microprocessor system in which scanning is controlled by easily-changed computer programs and CRT display to operators. A remote batch terminal accesses LBL-computers. Production is begining on 15 -foot BC film $(400 \mathrm{GeV}$ $\mathrm{pp)}$ for correlation studies involving $\mathrm{V}^{\mathrm{O}} \mathrm{s}$ and $Y^{\prime} s$.

\section{Goals and/or Major Results to Date}

(1) Our pioneering experimental work on inclusive reactions and Feynman scaling showed the ideas to be valid in general but not in all details. 


\section{UNIVERSITY OF CALIFORNIA}

Davis, California

(2) A technique that used the inclusive reaction at a single energy to obtain other quasi-two-body reaction energy dependences was developed.

(3) It was shown that, except for the low-multiplicity diffractive component, the distribution in multiplicity of $\pi^{-}$is Poisson-like at Fermilab energies and is independent of incoming particle nature at fixed energy.

(4) Mueller factorization was found to be valld willili a few perrent.

(5) The semi-inclusive correlation function was invented to separate production correlations from distribution correlations.

(6) A new $\mathrm{K}_{\mathrm{N}}(1760)$ resonance was discovered (with Purdue). $* * * * * * * * * * * *$

Title of Project: Theoretical and Phenomenological Analysis of High Transverse Momentum Phenomena, Inelastic Processes, New Particle Production and Particle Constituent Problems

Person in Charge: J. Gunion

Scientific and Professional Staff: J. Gunion

Scope of Work

The general purpose of my work is to develop the quark model in promising new directions and to pursue further existing applications of substantial importance. Of special interest to me are applications to high transverse momentum processes, bound state spectroscopy, chiral symmetry breaking, lepton deep inelastic scattering, massive lepton pair and charmed quark resonance production. In addition a general goal of recent work has been to understand within the quark framework standard, purely hadronic processes; the hope is that a unified description of many types of high energy interactions, with substantial predictive power, can be developed. Such extensions, by nature, require one to tackle questions involving the nature of quark confinement; in particular specification of the underlying field theory responsible for confinement is essential. My work has been in the framework of quantum chromodynamics (QCD), i.e. confinement in a color gauge theory. 


\section{UNIVERSITY OF CALIFORNIA \\ Davis, California}

\section{Goals and/or Major Results to Date}

Most recently, in collaboration with Stan Brodsky, we have shown that confinement by QCD allows one to understand the experimentally "established" universal nature of hadron production in (i) $e^{+} e^{-}$ annihilation, ( $(1)$ deep inelastic scattering $\left(\ell p \rightarrow \ell^{\prime} X\right)$, and (iii) hadron-hadron scattering. Our approach is based upon the idea that enforced separation of color results in bremsstrahlung or radiation of colored gluons which in turn produce hadrons. If the color quantum numbers responsible for hadron production are the same for all processes (in particular separation of a 3 and $\overline{3}$ of color dominates) then central hadron production, which depends only on color in our approach, will be universal. The color gluon model suggests that this control "plateau" region of hadron physics should rise with energy as experimentally observed. In addition fragmentation region behavior for all reactions, in particular hadron-hadron collisions, is specified and is in remarkable agreement with data. Indeed, we have managed to understand many features of hadronic data that have defied explanation in more conventional approaches (e.g. multiperipheral theory).

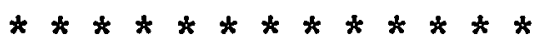


UNIVERSTTY OF CALIFORNIA

Irvine, California

Contract No. $\quad$ EY-76-C-0010, 019

Title of Project: Fundamental Neutrino Interactions

Person in Charge: Frederick Reines

Scientific and Professional Staff: C. Bratton, H.H. Chen, M.F. Crouch, H.S. Gurr, A.A. Hruschka, W.R. Kropp, J.F Lathrop, R.D. Newman, F. Reiness, H.W. Sobe1.

Scope of Work

Neutrino interactions of several fundamental types are under study at fission reactors (Savannah River $P l a n t$ ) and in cosmic iays. These include charged currents $\left(\nabla_{e}+d \rightarrow n+n+e^{+}\right.$, $\left.\bar{\nu}_{e}+p \rightarrow n+e^{+}\right)$neutral currents $\left(\nu_{e}+d \rightarrow n+p+\bar{\nu}_{e}\right)$, purely leptonic diagonal interactions $\left(\bar{\nu}_{e}+e^{-} \rightarrow \bar{\nu}_{e}+e^{-}\right)$and tests of $\bar{\nu}_{e}$ stability e.g. "oscillations" (departure of $\bar{v}_{e}$ flux from. $1 / \mathrm{R}^{2}$ variation with distance from reactor). Work is in progress in a search for double beta decay of ${ }^{82} \mathrm{Se}$. An investigation of the principal of baryon conservation and various cosmic ray backgrounds in a well shielded $7 \mathrm{~m}^{3}$ detector is underway in a salt mine near Cleveland. Development continues on techniques for ultra low level counting of radioactivity. Tests are being devised to search for an advanced potential in elestrnmagnetic. radiation and a departure from the inverse square law of gravitation at distances $<1$ meter. Feasibility studies are in progress for a facility at Fermilab to study $\nu_{\mu}+\mathrm{e}^{-} \rightarrow \eta_{\mu}+\mathrm{e}^{-}$ and other $\nu$ reactions possibly using a 11quid argon detector. The group is also concerned with establishing the feasibility of investigations of the use of very large water Cerenkov detectors $10^{4}-10^{10}$ tons, deep in the oceans for studies of supernovae bursts, high energy atmospheric neutrinos ( $\left.\gtrsim 10^{13} \mathrm{ev}\right)$ very high energy neutrinos from the metagalaxy (>10 $5^{15^{2}} \mathrm{ev}$ ).

Goals and/or Major Results to Date

After two decades of research, we have observed the process $\bar{v}_{e}+e^{-} \rightarrow \bar{v}_{e}+e^{-}$(Phys. Rev. Lett. 37, 315 (1976)). Observation of this fundamental, purely leptonic collision with properties so important to the weak interaction and astrophysics illuminates a previously unexplored aspect of the weak interaction. The detector consists of $\mathrm{NaI}$ and plastic scintillator elements connected by quartz windows to photomultiplier tubes, which pick up and amplify the scintillation light resulting from the collision between neutrino and target electron. The equipment is completely encased 
in lead, cadmium, liquid scintillation detector and water shielding. Neutrino interactions number about seven per day. A measure of the sensitivity of the detection system developed for the observation of elastic scattering is the astronomical value of the mean free path of the fission reactor antineutrinos used, 100,000 light years of liquid hydrogen. It is one of the exciting mysteries of science that something which reacts so little can have so much to do with the nature and structure of the universe.

$$
* * * * * * * * * * * * *
$$




\section{UNIVERSITY OF CALIFORNIA}

Irvine, California

Contract No: $\quad$ EY-76-S-03-0034,149

Title of.Project: High Energy Physics Study

Person in Charge: P. Condon

Scientific and Professional Staff: P. Condon, R. Burns, P. Cowel1

Scope of Work

The primary reocarch project of this contract is a search fon prompt production of single electrons in hadronic final states of electronpositron annihilation at SLAC-SPEAR. Th1s work is being done in collaboration with SLAC-Group $G$ and the UCLA contract headed by Harold Ticho. The experiment is designated SP-25 on the SLAC-SPEAR schedule and copies of the proposal are available from SLAC. The special responsiblity of this contract in the collaboration has been the design and construction of lead-plastic sandwich shower counters which will be used to identify electrons. These counters cover an area of about $500 \mathrm{ft}^{2}$, use 144 photomultiplier tubes and weigh over 15 tons. The experiment is scheduled to run in the first quarter of 1977.

Goals and/or Major Results to Date

Prompt single electrons in electron-positron annihilation are expected from the decay of charmed particles and of heavy leptons. Our experiment is designed to detect these electrons with good solid angle and high efficiency, and to determine the angular distribution and excitation function of their production with high statistics.

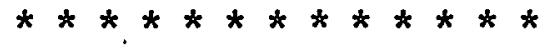

Title of Project: High Energy Physics Study

Person in Charge: J. Schultz

Scientific and Professional Staff: M. Mandelkern, L. Price

Scope of Work

The research involves a bubble chamber study of several processes occurring in antiproton interactions in deuterium from rest to just below $1 \mathrm{GeV} / \mathrm{c}$ lab momentum. Film being used includes exposures from the Brookhaven National Laboratory 30-inch and Argonne National Laboratory 12-foot bubble chambers. Of principal interest is a search for resonances or bound states near threshold in the 


\section{UNIVERSITY OF CALIFORNIA \\ Irvine, California}

nucleon-antinucleon system. Several theoretical approaches suggest the possibility of a large number of states in this region, and recent experiments performed elsewhere on pd interactions have yielded suggestive evidence of a pn bound state at approximately $1790 \mathrm{MeV}$ and narrow resonances at 1897 and $1932 \mathrm{MeV}$. In addition, an observed excess of detected $\gamma$-rays in $\bar{p} d$ annihilations at rest is indicative of possible electromagnetic transitions between states in the $\bar{p} N$ system. Our aim is to extend these investigations, attempt to confirm the existence of these and/or other states, and to obtain additional information on the quantum numbers of these states. A study of neutral strange particle production in $\bar{p} d$ annihilations is also in progress in order. to (i) compare results with those found previously in our studies of : $\bar{p} p$ annihilations, and ( $i i)$ investigate the mechanism of $\Lambda$-production which necessarily involves both nucleons of the target deuteron.

\section{Goals and/or Major Results to Date}

Using the relatively good $\gamma$-ray conversion efficiency of the large ANL chamber, we intend to study the $\gamma$-ray spectrum accompanying $\bar{p} d$ annihilations as a probe of possible bound states. Events with $\gamma^{\prime}$ s will also yield a determination of neutral pion production in final states not otherwise accessible. Annihilations with recoll protons will be analyzed to search for states manifested as peaks in the spectator momentum distribution, and to determine the characteristics, e.g. quantum numbers, of the multipion systems resulting from the decays of any such states. Production of neutral strange particles will be studied to explore resonance production and annihilation mechanisms in $\bar{p} d$ interactions. There are no final results to report on these projects at this time.

$$
\star * \star * \star * * * * * * * * *
$$


UNIVERSITY OF CALIFORNIA

Los Angeles, California

Contract No. EY-76-C-03-0010, 017

Title of Project: Research in High Energy Physics

Person in Charge: D.H. Stork, H.K. Ticho

Scientific and Professional Staff: D.H. Stork, H.K. Ticho, W.E. Slater, C. Buchanan, J. Hauptman, L. Nodelman

Scope of Work

1) Measurement of the Pion Form Factor (Fermilab experiment E216). A direct measurement of the pion form factor up to $q^{2}=0.080$ $(\mathrm{GeV} / \mathrm{c})^{2}$ has been made by scattering $100 \mathrm{GeV}$ pions on electrons in a liquid hydrogen target. Preliminary results indicate that the rms pion radius is $0.55 \pm 0.06 \mathrm{f}^{2}$.

2) Measurement of the Kaon Form Factor (Fermilab experiment E456). The first measurement of the kaon form factor is being carried out with a high-resolution, narrow-angle spectrometer by scattering $250 \mathrm{GeV}$ kaons on electrons in a liquid hydrogen target.

3) Charged Baryon Exchange in $12 \mathrm{GeV} \pi^{-} p$ Reactions (SLAC experiment BC58). This experiment has been carried out with the SLAC 40" rapid cycling bubble chamber by triggering on fast forward neutrons in a neutron calorimeter and observing the neutron interaction point in optical spark chambers. The data exhibit striking baryon exchange characteristics.

4) Antibaryon Inclusive Reactions in $e^{+} e^{-}$Reactions (SLAC experiment SP10). Annihilation packages were added to two sides of the Magnetic Detector at SPEAR. Data collected over the past year are being studied for inclusive features which characterize the baryon inner structure and are being studied for evidence of charmed particles.

5) Direct Electron Detector for $\mathrm{e}^{+} \mathrm{e}^{-}$Rẹactions (SLAC experiment SP25: DELCO). Direct electrons from $\mathrm{e}^{+} \mathrm{e}^{-}$annihilation at SPEAR will be detcctcd in a unique dcvice featuring a Cerenkov counter with a large solid angle and a high background rejection. Large yields from charmed particle decays are expected and the heavy lepton question should be clarified.

6) Proposal for a T1me Projection Facility at PEP. We are developing with LBL and others this proposal for particle detection and identification with a time projection chamber 


\section{UNIVERS ITY OF CALIFORNIA \\ Los Angeles, California}

in a superconducting solenoid magnet surrounded by a liquid argon photon detector and an iron muon detector.

\section{Goals and/or Major Results to Date}

We seek an understanding of the nature of the particles of high energy physics: of their properties, inner structure, and interactions. At Fermilab we are investigating the electromagnetic structure and size of pions and kaons by studing elastic scattering of high energy pions and kaons on electrons. At SLAC we have determined the characteristics of charged baryon exchange in $12 \mathrm{GeV}$ $\pi^{-} p$ interactions. With high energy $e^{+} e^{-}$annihilations at SPEAR we are studying nucleon structure by means of inclusive antinucleon reactions, will study the direct electron decays of charmed particles, and will clarify the question of heavy lepton existence.

$$
* * * * * * * * * * * * * *
$$


UNIVERSITY OF CALIFORNIA

Riverside, California

Contract No. EY-76-S-03-0034, 107

Title of Project: New Particle Searches at the CERN ISR

Person in Charge: A. Kernan

Scientific and Professional Staff: J. Eickmeyer, A. Kernan, B. Shen,

G. Van Dalen, R. Wojslaw

Scope of Work

This University of California, Riverside, project is carried out at the CERN ISR in collaboration with CERN and the Universities of Aachen, Harvard, and Munich. The collaboration is curieully setting up a multiparticle magnetic spectrometer covering the forward angular cone out to $40^{\circ}$. A toroidal magnetic field is generated by a set of 12 air coils arranged like the spokes of a whee 1 around the beam axis. Gas Cerenkov counters fill the surface between adjacent spokes of the magnet. These counters, in conjunction with lead - scintillator shower counters and:timeof-flight measurement, provide electron and hadron identificalion. Particle trajectories are reconstructed with high resolution, $(\sim 0.1 \mathrm{~mm})$ drift chambers. Installation of this detector at intersection 6 of the CERN ISR will be completed hy Novambor 1976 .

Goals and/or Major Results to Date

First application of this device will be the study of (i) charmed particle and (ii) direct electron pair production in strong interactions. The detector will also be complemented by a high precision magnetic spectrometer at $180^{\circ}$, allowing us to investigate the structure of diffractively produced high mass objects. This process, apart from its intrinsic interest, is a possible source of charmed particle pairs. The addition of a muon telescope to the setup 1s envisaged for spring 1977; this will increase the flexibility of our setup, and in particular will enable us to extend current studies of H-e pair production. Finally this detector will provide for the first time detailed information on cluster composition in proton proton interactions at the highest energies.

$$
* * * * * * * * * * * * * * * *
$$


UNIVERSITY OF CALIFORNIA

Santa Barbara, California

Contract No. EY-76-C-03-0010-0023

Title of Project: User Group at UCSB

Person in Charge: D. Caldwell

Scientific and Professional Staff: R. Morrison, A. Eisner, S. Yellin, A. Lu

Scope of Work

At present we are finishing the analysis of two SLAC experiments, taking data at Fermilab, and planning future experiments at SLAC and PEP. The SLAC work being analyzed includes deep inelastic $\pi^{\circ}$ electroproduction, a comparison of $\mathrm{e}^{+}$and $\mathrm{e}^{-}$electroproduction of high energy photons (hereafter referred to as the 3- $\gamma$ experiment). The computational work at SLAC is nearing completion and some physics results (see below) have been obtained.

At Fermilab we-have completed and published measurements of $\mathrm{J} / \Psi$ photoproduction and now we are measuring photon total absorption cross sections in the world's highest energy tagged photon beam. We plan to cover photon energies from 20 to $270 \mathrm{GeV}$. Hydrogen cross sections are to be measured to better than $1 / 2 \%$, and at least over the lower half of the energy range, neutron cross sections to measure photon shadowing at several energies.

Some effort is being devoted to proposing both a more complete version of the 3- $\gamma$ experiment at SLAC and, with the University of California at San Diego and Davis groups, a two-photon experiment at PEP.

Goals and/or Major Results to Date

Results from the deep inelastic $\pi^{\circ}$ electroproduction experiment include tests to distinguish between the two ways in the parton model in which the virtual photon could interact with a nucleon:

1) By dissociating into a parton-antiparton pair, which then produces two "jets" upon interaction; or

2) By directly hitting a parton in the nucleon, producing a single jet. 


\section{UNIVERSITY OF CALIFORNIA}

Santa Barbara, Callfornla

Although we had evidence for the first type of process for real photons, we apparently see the second type for virtual photons: For the $e^{+} e^{-}$experiments we see to an accuracy of $1 / 3 \%$ no difference In $e^{t}$ and ' $e^{-}$deep inelastic scattering, but that channel in which a high energy photon is produced ylelds a difference of $\sim 10 \%$, an effect understandable at present only in the parton model.

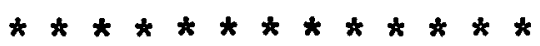


UNIVERSITY OF CALIFSKNIA

Santa Cruz, Cal i fornia

Contract No. EY-76-S-03-0034, 197

Title of Project: Experimental Research in High Energy Physics

Person in Charge: Clemens A. Heusch

Scientific and Professional Staff: C. del Papa; D. Dorfan, S. Flatte, T. Schalk, A. Seiden, D. Smith

Scope of Work

1) Investigation of hadronic final states in deeply inelastic muon-proton and muon-neutron scattering. Determination of multiplicities, topologies, forward charge ratios, energy and momentum distributions. Elastic and inclusive production of vector mesons; strange particle production. Search for excited muon states.

2) Search for narrow hadronic states with new quantum numbers. Study of production in $\pi^{+} p$ collisions and decay inside a streamer chamber volume, of high-mass states that may be interpretable in terms of the charm quantum number. Triggering by single or double muons from interaction.

3) Scattering, both elastfc and inelastic of very-high-energy photons. Determination of forward Compton amplitudes, inclusive production of pseudoscalar or vector mesons with decay into gammas or electrons. Search for photoproduction of new heavy particles decaying into showering final states.

Goals and/or Major Results to Date

1) Universal dependence of multiplicity of charged hadrons on available energy. Independence of fractional topologies on momentum transfer $Q^{2}$. Constancy of relative abundance of elastic vector mesons.

2) Production limits for charmed hadrons decaying into 2- to 6 charged particles, produced in $\pi p$ collisions. Trigger on one or two munn candidates.

3) Investigation of forward dispersion relation, measurement of quark charges. Production of new showering particles.

$* * * * * * * * * * * * * *$ 


\section{UNIVERSITY OF CINCINNATI \\ Cincinnati, Ohio}

Contract No. EY-76-S-02-29.78

Title of Project: Inelastic Strong Interactions at High Energies.

Person in Charge: P. Suranyi

Scientific and Professional Staff: P. Suranyi, J. Scanio

Scope of Work

Strong interactions are studied using the methods of Reggeon Field Theory (RFT). Special emphasis is made to go beyond the conventional $\varepsilon$-expansion results. Objects to be achieved include the determination of critical parameters, scaling functions (including that of the multiplicity distribution), the low multiplicity behavior of which can be compared with the results of Bartels and Rabinovici (Phys. Rev. D12, 3938 (1975) obtained by different methods. Another aim of our investigation is the study of the s-channel unitarity properties of RFT.

We wish to make use of the lattice model of Cardy and Sugar (Phys. Rev. D12, 2514 (1975) for the reasons of the questionable value of $\varepsilon$-expansion result. Multiplicity distributions are going to be studied with the help of the cut RFT (P. Suranyf, Phys. Rev. D12, 3938 (1975)).

Goals and/or Major Results to Date

We proved the divergence of the perturbation expansion in Reggeon Field Theory (RFT) casting a doubt to the meaningfulness of results obtained with the help of e-expansion in a paper under publication. In another paper just submitted for publication the existence of phase transitions was proved in RFT using the lattice model of Cardy and Sugar. The vacuum expectation value of the Reggeon Field was shown to be zero below and nonzero and time dependent above a critical coupling. 
UNIVERS ITY OF COLORADO

Boulder, Color ado

Contract No. EY-76-C-02-2114

Title of Project: Study of $\mathrm{K}_{\mathrm{L}}^{\mathrm{O}}$ and Photon Interactions

Person in Charge: Uriel Nauenberg

Scientific and Professional Staff: D. Bartlett, W. Ford. G. Schultz, D. McQuate, M. Mugge

\section{Scope of Work}

We are initiating a study of strong interactions of $\mathrm{K}_{\mathrm{L}}^{0}$ mesons and photoproduction experiments at Fermilab. We are presently lesigning a high intensity, high energy $\mathrm{K}_{\mathrm{L}}^{\mathrm{O}}$ and $\psi$ : beam with a very low neutron background. This very useful beam of neutral $\mathrm{K}$ mesons and photons is produced when a pion beam strikes a target. Unlike such beams when produced by a proton beam, this beam will have a very low neutron content which allows one to study the interactions of $\mathrm{K}$ mesons and photons without any background associated with neutron interactions. In fact, preliminary calculations show that in the high momentum region of the beam spectrum the $K_{T}^{o}$ to neutron ratio could be as high as 10 to 1 . We plan to use this beam to study the interactions of $\mathrm{K}_{\mathrm{L}}^{o}$ and $y^{\prime} s$. The interactions of $\mathrm{K}_{\mathrm{L}}^{\mathrm{O}}$ mesons is a poorly known subject at present.

\section{Goals and/or Major Results to Date}

We are presently carrying out preliminary measurements to determine the fluxes of $\mathrm{K}_{\mathrm{T}}^{\mathrm{O}}$, neutrons and gammas associated with such a beam. Our goal is to have these results soon and have a full proposal submitted for review by the program advisory committee for either the fall or spring meeting.

$$
* * * * * * * * * * * * * * *
$$

Title of Project: Elementary Particles and High Energy (Theory)

Person in Charge: K. T. Mahanthappa

Scientific and Professional Staff: K. T. Mahanthappa, D. Unger, J. Crawford

\section{Scope of Work}

We are pursuing two different problems: a) There is much speculation concerning quark confinement and very little concrete work has been done in the framework of realistic models. The problem essentially 


\section{UNIVERSITY OF COLORADO \\ Boulder, Colorado}

involves strong coupling. It could possibly be that there is a. phase transition from quark phase to no-quark but bound state phase when strong coupling is present. We have been investigating such. a possibility starting. with the generating functional for Green's functions. We are at present considering the case of a real scalar field with self-interaction. Our technique invclves performing certain functional integrations exactly and expanding in terms of $1 / \mathrm{g}^{2}$. We are at present looking into the renormalizability of the program. b) At present, the only models which are not in contradiction of experimental data of neutrino scattering are those with five or six quarks. We are investigating the possibility of the enhancement of $\Delta I=\frac{1}{2}$ in non-leptonic weak decays in these models using the operator product expansion and renormalization group methods. We are especially interested in finding out whether one can account for all of the observed decay rates in these models.

\section{Goals and/or Major Results to Date}

a) During the past year we have shown that the Yang-Mills gauge field theoretical models of elementary particles with symmetry breaking via the Higgs mechanism could have their genesis in more fundamental theories which are symmetric and do not contain elementary scalar fields. Our results clearly demonstrate the possibility of having an asymptotically free Yang-Mills theory of strong interactions in which all gauge bosons (vector gluons) acquire mass via dynamical symmetry breaking. Also we were able to show that the physical mass of a fermion is dynamirally generated In symmetric Yang-Mills theories (Phys. Rev. D 13, 2907 and 2910 $(1976))$. b) We investigated the effect of the energy loss caused by neutrino pair emission due to the existence of neutral currents on the evolution of stars and found that the effects are not significant at stellar temperatures $\sim 10^{90} \mathrm{~K}$ (Astrophysical Journal 206, 208 (1976)).

$$
* * * \star * * * * * * * * * *
$$

Title of Project: Tachyons and Monopoles

Persun In Charge: D. Bartlett

Scientific and Professional Staff: G. Schultz, D. Soo

Scope of Work

We have been using the extensive fringing magnetic field of Fermilab's 15-foot bubble chamber to attract and accelerate magnetic monopoles 


\section{UNIVERSITY OF COLORADO \\ Boulder, Colorado}

which may exist in cosmic rays. For the past two years in collaboration with $M$. White (Princeton) we have searched for a north magnetic monopole which goes faster than light ("tachyon monopole"). Our detector is a room-sized box mounted above the bubble chamber. Photomultiplier tubes mounted on the corners of the box record the Cerenkov radiation which a tachyon monopole should emit even in vacuum. We have not found any tachyon monopoles and are now changing our apparatus so as to be sensitive to ordinary (slower-than-light) monopoles. The section of roof above our box is to be replaced by a large roof scuttle which will be opened in fair weather to let the monopoles reach the floor of the box where they will be recorded by tracks left in Lexan plastic sheets. The sensitivity of this arrangement is greatly enhanced by the tendency of slow monopoles to follow lines of magnetic field and thus be funnelled into our apparatus.

Goals and/or Major Results to Date

Our tachyon monopole detector has failed to record any events in an exposure of 50 days. The flux of sych particles in cosmic rays cannot exceed $2.5 \times 1 \sigma^{-15} \mathrm{~cm}^{-2} \mathrm{sec}^{-1}$ if they follow the earth's magnetic field lines or $1.2 \times 10^{-12} \mathrm{~cm}^{-2} \mathrm{sec}^{-1}$ if they do not. In either event this limit is at least 400 times lower than that inferred from a previous measurement.

We plan to install the roof scuttle this fall. With this modification, the existing limit on in-flight detection of monopoles can be lowered by a factor of 24 in a seven month run.

$$
* * * * * * * * * * * * *
$$

Title of Project: Filementary Particles and High Energy Phenomena

Person in Charge: U. Nauenberg, D. Bartlett, W. Ford

Scientific and Professional Staff: U. Nauenberg, D. Bartlett, K. Mahanthappa, W. Ford, J. Smith

Ecope of Work

For the next year our goals are the following:

1) To complete the measurement of neutral particle fluxes in a pion-produced $K_{0}$ beam and use the results for a proposed neutronless $\mathrm{K}_{\mathrm{o}}$ beam at Fermilab. 


\section{UNIVERSITY OF COLORADO}

Boulder, Colorado

2) To complete the analysis of dimuon events produced in the iron ball experiment at SPEAR.

3) To accumulate data in an exposure of Lexan detectors to magnetic monopoles above Fermilab's fifteen-foot bubble chamber.

4) To build large Cerenkov counters and proportional chambers for the tagged photon facility at Fermilab.

Our theoretical investigations will support these activities. In addition, we will study the behavior of renormalization constants for bound states.

Goals and/or Major Results to Date

In the decay of the neutral kaon we have found:

1) That the CP-violating parameter, $\varepsilon$, is about $10 \%$ higher than previously determined;

2) That CPT is conserved to within $2 \%$;

3) That a quadratic term is needed in the matrix element for $\mathrm{K}_{\mathrm{L}}(\pi 3)$ decay;

4) That the $\Delta I=1 / 2$ rule is violated in this decay.

18 We have also found no evidence for the production of tachyon monopoles near a nuclear source or in cosmic rays. We have shown that scaling can be used to predict particle production distributions of higher energies.

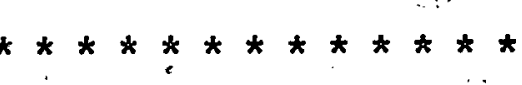


COLUMBIA UNIVERSITY

New York, New York

Contract No. $\quad$ EY-76-C-02-2271

Title of Project: Theoretical High Energy Physics

Person in Charge: T. D. Lee, R. Serber

Scientific and Professional Staff: N. Christ, G. Feinberg, H. Foley, R. Friedberg, A. Mueller, G. Wick, J. Finkelstein, E. Weinberg, A. Guth, J. Koplik

Scope of Work

Our main objective is to concentrate on various fundamental theoretical aspects of high energy physics.

At present, we are particularly interested in the following subjects:

1) The origin of symmetry violation and the structure of the physical vacuum;

2) The possible existence of an entirely new family of stable super-heavy nuclei, called abnormal nuclear states;

3) The quantization of soliton solutions in a relativistic local field theory and the possible description of all observed hadrons as solitons;

4) Magnetic monopoles and their properties;

5) Properties of $\mathrm{J} / \Psi$ mesons and other newly found narrow resonances;

6) Strong interaction theories.

In the work of Lee and Wick, the vacuum is viewed as a dynamic entity which provides the origin of symmetry breaking. On the other hand, by changing some external variables it is possible to alter the properties of the vacuum state, and thereby to restore the broken symmetry. One of the consequences of such theoretical speculation is the possibility of abnormal nuclear states. The important question of whether they can be produced in a heavy ion collision in the future or whether they may already exist in nature is currently under study.

The derivation of quantum soliton solutions represents a major recent progress in theoretical physics. These solutions can be classified into two types, topological and nontopological. The former refer to all magnetic monopole solutions, and the latter we hope will eventually lead us to a correct description of all hadrons. 
COLUMBIA UNIVERSITY

New York, New York

A general quantization method of solitons has been developed by Christ and Lee. Specific solutions in four space dimensions have been derived by Lee, partly in collaboration with Friedberg and Sirlin, for a wide variety of relativistic renormalizable local field theories. These solutions are being extended to include quark fields. It is hoped that eventually all hadrons can be understood as solitons, while all leptons are described by the usual perturbative series around plane wave solutions.

The Columbia Theoretical Group has traditionally had a close relationship with the various experimental groups at Nevis, Brookhaven. Fermilab, SLAC, LBL, and CERN. Many of the experiments done in these laboratorles are direct offshoots of discussions between our members and the experimentalists.

Goals and/or Major Results to Date

To date, significant results have been obtained in abnormal nuclear states, vacuum structure, and soliton solutions, as mentioned above. Various phenomenological analyses of $\mathrm{J} / \Psi$ mesons have been made by Feinberg and others. These investigations will, of course, be extended to the newly found resonances at SPEAR and those discovered by the Columbia/Fermilab collaboration. The recent work of Mueller examines the foundation of the string model, and his paper with Koplik gives detailed predictions of meson production in high energy nuclear collisions. Various aspects of the multiperipheral model have been studied by Finkelstein and Koplik; those of the Bethe-Salpeter equation in the large $Q^{2}$ region have been discussed by Guth and others. In the future, we plan to maintain both the depth and the breadth which characterize our present program. 


\section{DUKE UNIVERSITY \\ Durham, North Carolina}

Contract No. $\quad$ EY-76-S-05-3065

Title of Project: The Study of the Interactions between Elementary Particles and their Interactions with Nuclei

Person in Charge: W. Walker

Scientific and Professional Staff: L. Fortney, A. Goshaw, J. Loos, W. Walker, J. Lamsa, W. Robertson

\section{Scope of Work}

We have been studying the interactions of high energy pions with protons and nuclei. The purpose of the work is to gain a broad understanding of particle production at high energy. The use of a nuclear target allows one to gain insights as to the development of particle production at very small distances and very short times. We are also investigating particle production by diffraction dissociation primarily at Fermilab energies. We will start an experiment to investigate the source of directly produced high energy electrons. This investigation will also be a useful adjunct to our recently concluded energy balance experiment. We also hope to start further studies of the interaction of high energy pions with a heavy nucleus such as lead or uranium.

\section{Goals and/or Major Results to Date}

We have recently concluded a very comprehensive study of the collision of $10.5 \mathrm{GeV} / \mathrm{c}$ pions.with Neon nuclei. These data give a remarkably comprehensive picture of these collisions, by far the best ever obtained. We have also concluded a detailed study of inclustve $\pi^{\circ}$ production--again by far the most detailed ever done. An experiment has been completed which explored the possibility of the production of as yet undiscovered long-lived neutral particles. Such particles carry less than $3 \%$ of the outgoling wumentum.

$$
* * * * * * * * * * * * *
$$


FLORIDA STATE UNIVERSITY

Tallahassee, 'Florida

Contract No. EY-76-S-09-3509

Title of Project: High Energy Particle Physics - Experimental

Person in Charge: $\mathrm{J}$. Albright, $\mathrm{v}$. Hagopian, J . Lannutti

Scientific and Professional Staff: J. Albright, R: Diamond, S. Hagopian, V. Hagopian, J. Lannutti, W. Morris

Scope of Work.

1) Bubble chamber experiment with $\pi^{+} \mathrm{d}$ at $15 \mathrm{GeV} / \mathrm{c}$ in the SLAC 82" chamber consisting of yơk pictures: Appiuxillately $85 \mathrm{~K}$ otrange and non-strange events have been measured. Analysis is continuing of various multiparticle final states, $\pi-\pi$ resonance effects, forward scattering amplitudes of various final states to understand exchange mechanisms, comparisons of inclusive data to check for scaling, strange particle final states to search for charm, etc::

2) Bubble chamber experiment with $\pi^{-} p$ at $250 \mathrm{GeV} / \mathrm{c}$ in the FNAL $15^{\prime}$ chamber consisting of $46 \mathrm{~K}$ pictures: approximately $3.5 \mathrm{~K} \mathrm{~V}$ ' s have been measured and the associated primaries are in process. Currently electron pair gamma conversions are being measured. Analysis is continuing of charm possibilities, cluster effects with strange particles, inclusive strange particle and photon production. Measurements will be made of low multiplicity events, diffractive dissociation of pion," e.tc .

3) Bubble chamber experiment with $\pi^{-p}$ at $360 \mathrm{GeV} / \mathrm{c}$ in the FNAL 15' chamber has been approved for $200 \mathrm{~K}$ pictures, but only $20 \mathrm{~K}$ pictures have been obtained. Most of the scanning on this so far has been done by collaborators. Measurements have also begun along the lines discussed above.

4) BNL MPS search for $\equiv^{*}$ states using $\mathrm{K}^{-}$beam and recoil $\mathrm{K}^{+}$trigger. Currently commissioning and testing small $\mathrm{K}^{+}$delector already available and designing larger $\mathrm{K}^{+}$detector. Run expected in fall 1977.

Goals and/or Major Results to Date

1) Published $\pi^{+} d$ results to date: $\Delta-\Delta$ composition of the deuteron has been measured; $\mathrm{K}_{\mathrm{S}} \mathrm{K}_{\mathrm{s}}$ resonance effects at low $t$ have been studied; search for charm among strange particle events set limits on production and found, one long-lived $K i f$ andidate at the expected mass; analysis of the $3 \pi$ d final state showing appreciable spin flip $f_{0}$ exchange for 3 f production. 
FLORIDA STATE UNIVERSITY

Tallahassee, Florida

2) Pub1ished $\pi^{-} p$ results to date: set limits on charm production among 'strange particle events; inclusive study of $K_{S}$ and $\Lambda$ production; preliminary multiplicity study fits KNO scaling.

3) Goals: In addition to ${ }^{*}$ search, work will continue on f $^{-} p$ $360 \mathrm{GeV} / \mathrm{c}$ and strange particle beam studies for FNAL and CERN.

$$
* * * * * * * * * * * * *
$$

Title of Project: High Energy Particle Physics - Theoretical

Person in Charge: J. Kime1, P. Williams

Scientific and Professional Staff: J. Kimel, J. Owens, P. Williams, D. Gluch

Scope of Work

The major focus of the particle theory group is on hadron dynamics and phenomenology. Emphasis recently has centered on partial wave analyses of three-body final states, diffraction dissociation and Deck models, amplitude analysis of multi-body final states, studies of inclusive reactions in the triple-Regge region, and the phenomenology of quark-parton models. The theoretical work is usually directly related to experiment; and the close rapport between theorists and experimentalists at FSU, a strengthening factor we believe, is characteristic of the group. This rapport is a major factor in the development of the whole particle physics program at FSU (well exemplified by our collaborative work in the area of $\pi-\pi$ physics) and is expected to contimue.

\section{Goals and/or Major Results to Date}

(Summer 1975 through Summer 1976)

1) We have completed the partial wave analysis, consistent with unitarity, of the $\mathrm{K}^{-} \mathrm{n} \rightarrow \Sigma^{+} \pi^{-} \pi^{-}\left(\Lambda^{-} \pi^{0}\right)$ final states.

2) Investigation of diffractive inelastic processes has resulted in (experimentally saturated) model bounds on slopes based on factorization of the $s$ - and $t$-channel partial wave amplitudes.

3) Analysis of the reaction $\pi^{+} d \rightarrow \pi^{+} \pi^{-} \tilde{r}^{-} \mathrm{d}$ has shown clearly the need for a $\rho$ contribution in addition to the usual $\pi$-exchange Deck model. 
4) A triple-Regge analysis of the reaction $p \dot{p} \rightarrow \Delta^{++}+X$ has established that absorptive corrections must be applied to $t$ he trlple-Regge pole terms if the correct normalization is to be achièved.

5) The apparent weakening of the pion"cut in the reaction $\pi^{-} p \rightarrow \pi^{+} \pi^{-} \mathrm{n}$ has been studied and resolved.

$$
* * * * * * * * * * * * * * *
$$

Title of Project: High Energy Particle Physics - Gravitational Theory

Person in Charge: P. Dirac

Scientific and Professional Staff: P. Dirac, L. Halpën

Scope of Work

Main work is development of the theory of varying $G$, the universal gravitational constant. Old work on magnetic monopoles is being re-examined and developed further in view of recent claims of monopole observations. Study of superfluids in gravitational fields is in progress. Extension of the Gauge Principle of Dirac's Equation of the spinning electron to the general invariance group of this equation and its Yang-Mills fields:

\section{Goals and/or Major Results to Date}

Small workshop on the experimental observation of gravitation was held in November 1975 with five experimental groups represented. The proceedings have been prepared and a publisher is being sought. $P$ aper on method for analysing data to observe $\ddot{G}$ variation has been published. New book on General Relativity has been published. Paper has been completed on an approach to a unifled treatment of electromagnetic and gravitational theory emerging from the covarlant Dirac equation. Paper has been completed on gravitational and Inertial effects on superconducting shielding. Paper hà been completed on conclusions, from the extended gauge principle of Dirac'o equarion.

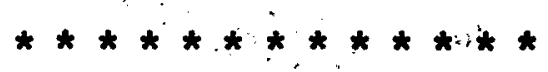


UNIVERSITY OF GEORGIA

Athens, Georgia

Contract No. ... EY-76-S-09-0946

Title of Project: High Energy Hadron-Hadron Collisions

Person in Charge: T. Chou

Scientific and Professional Staff: T. Chou

Scope of Work

The research program at Georgia centers on the study of hadron-hadron collisions. The program is being developed along the general direction of further exploiting the geometrical picture. Specific items now under investigation are the following:

1) The energy dependence of the elastic scattering model. Emphasis is on determining whether the opacity of the pp system favors factorization or geometrical scaling.

2) Impact parameter description of multiplicity fluctuations. It is our intention to construct explicit models for discussing the average multiplicity distribution and its fluctuations by folding simple dynamics with geometrical notions, and to give more precise quantitative predictions that can be compared with experiments.

3) Determination of the velocity profile inside a nucleus. The concept of the velocity distribution of the hadronic stuff inside a hadron is generalized to polarized nuclei. The nucleonic current density can be computed using nuclear theory. The value of the polarization rotation parameter for several light nuclei can be obtained.

4). Appiication of the geometrical approach to elastic diffraction processes. Preliminary studies of the t-dependence of the differential cross section for inelastic diffraction processes using the geometrical model yields satisfactory results.

Goals and/or Major Results to Date

Results of an extensive phenomenological analysis of the recent elastic pp scattering data seem to support factorizable eikonal models on energy dependence of opacity and are in disagreement with the geometrical scaling hypothesis. Details will be published.

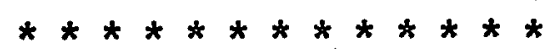




\section{HARVARD UNIVERSITY \\ Cambridge, Massachusetts}

Contract No. EY-76-S-02-3227

Title of Project: The Theory of High Energy Collision Processes

Person in Charge: T. T. Wu

Sclentific and Professional Staff: B. McCoy

Scope of Work

Because of the many desirable properties, such as satisfying unitarity and having particle production, of relativistic quantum fleld theory (RQFT), it is the underlying idea of this program to study the highenergy behavior of RQFT; and to apply the general features of the result to strong interactions. One of the early predictions of this procedure was the rising total cross section, and was first experimentally verified several years later at ISR for proton-proton scattering. Using this ISR data, a phenomenological model was developed to predict the rise of the $\pi^{ \pm} \mathrm{p}$ and $\mathrm{k}_{\mathrm{p}}^{ \pm}$total cross section, and these predictions were quantitatively verified by experiments at Fermilab. Another prediction from RQFT was the rising plateau for pionization, and was accurately verified this year at ISR.

More recent work on the high energy behavior of two-body processes deals with, among others, Yang-Mills fleld and quantum number exchange. It is found that the various two-body processes have much more in common than previously expected. Furthermore, this work puts sharply in focus the necessity of obtaining a deeper understanding of production processes. This study of production processes, together with the related problem of constructing models that incorporates as many as possible of the features learned from RQFT, is the major ongoing activity in this program.

Goals and/or Major Results to Date

Goal: To understand some aspects of hadronic processes at high energies of the basis of quantum field theory.

Major results: (Mostly carried out in collaboration with Prufessors Hung Cheng, Barry M. McCoy, James Walker, and Chen Ning Yàng)

\section{A) Theory}

1) Pomeron above 1;

2) Cross sections for pionization;

3) High-energy behavior of Yang-Mills theory in sixth order;

4) High-energy behavior to all orders of fermion exchange in QED. 
HARVARD UNIVERSITY

Cambridge, Massachusetts

B) Phenomenology

1) Rising total cross sections;

2) $\mathrm{Re} / \mathrm{Im}$ for forward amplitude;

3) Exclusive pionization;

4) Rising plateau of pionization;

5) Photoproduction of $\phi$.

C) Theory not directly related to this program

1) Section formulation of gauge fields;

2) Properties of the magnetic monopole;

3) Feynman rules for Yang-Mills field.

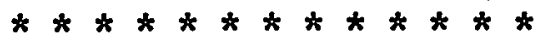


UNIVERSITY OF HAWAII .

Honolulu, Hawai i

Contract No. EY-76-C-03-0511

Title of Project: Research in High Energy Physics (Experimental Progr am)

Person in Charge: V. Peterson, R. Cence

Scientific and Professional Staff: R. Cence, F. Harris, M. Jones, T. Katsura, S. Parker, M. Peters, V. Peterson, V. Stenger, D. Yount

Scope of Work

Experimental research in high energy physics with emphasis on studies of the weak interactions using neutrino beams and $e^{+} e^{-}$ colliding beams from particle accelerators. The major effort is. currently directed to bubble-chamber experiments at Fermilab, using both hydrogen and neon-hydrogen liquid fillings. A special MWPC array, developed by our Hawaii-LBL team, forms an "External Muon Identifier" (EMI) for the $15^{\prime}$ foot bubble chamber. The Hawaii group is presently. involved (with three other groups.) in analyzing BC-EMI data from three major runs of the Fermilab hybrid system:. E-45, neutrinos on hydrogen; E-28, neutrinos on "light" neonhydrogen $(23 \% \mathrm{Ne})$; and E-172, antineutrinos on "heavy" neonhydrogen $(64 \% \mathrm{Ne})$.

Film analysis facilities, including scanning and measuring equipment, comprise the major installation at Hawaii. In addition, the campus computer is used extensively for data analysis.

The Hawaii experimental group is also involved in a big experiment (SP-26) at the SPEAR colliding beam storage ring. The experiment is to study $e-\mu$ production, and other electron/photon final states, with the Magnetic Detector improved by adding a Lead-Glass "Wall" for better electron detection.: This experiment is just beginning (September 1976).

Goals and/or Major Results to Date

The basic goal is to understand the nature of the weak interaction between elementary particles.

The neutrino bubble-chamber experiments have provided several interesting results to date. The most interesting result $h_{\text {as }}$ been the clear discovery of electron-muon "dilepton" events (E-28 experiment) which could best be explained by invoking 


\section{UNIVERSITY OF HAWAII \\ Honolulu, 'Hawaii}

"charmed quarks." We are now searching for muon-muon dileptons in both E-28 and E-172 in order to check the results of earlier counter experiments. Tests of scaling have been made with the "clean" data from E-45 (pure hydrogen target).

$$
* * * * * * * * * * * * * *
$$

Title of Project: Research in High Energy Physics (Theory Program)

Person in Charge: S. F. Tuan

Scientific and Professional Staff: P. Dobson, Jr., S. Pakvasa, W. Simmons, H. Sugawara, S. Tuan, J. Okada, and D. Roberts

\section{Scope of Work}

The scope of work during the past year has been from catastrophe theory to phenomenology. Specifically, the areas attacked are phenomenology of $\Psi, \Psi^{\prime}$ decays, phenomenology of neutrino interactions, production mechanisms. for new heavy particles, new gauge models of many quarks, localization of quarks, and dual string models.

\section{Goals and/or Major Results to Date}

Our major goals and results are: a) a complete and comprehensive analysis of the six quark vector model (competitive with similar work done at Harvard, Princeton, and $\mathrm{Cal}$. Tech) including all experimental implications. We were the first anywhere to propose how to incorporate $C P$ effects into such a picture; b) a thorough and authoritative account on how thermodynamics can be used in analyzing the central production of the psions and $\phi$ particle which obey Zweig suppression; c) our probe concerning the increasing use fulness of the modified Kuti-Weisskopf quark parton distributions (developed here) in an array of recent high energy experiments.

$$
* * * * * * * * * * * * * *
$$


INDIANA UNIVERS ITY

Bloomington, Indiana

Contract No. EY-76-S-02-2009

Title of Project: Indiana High Energy Physics Group

Person in Charge: H. Neal, R. Heinz

Scientific and Professional Staff: B. Brabson, R. Crittenden, A. Dzierba, H. Ogren, S. Gray, J. Sauer, D. Rust

Scope of Work

During the period December 1, 1976 through November 30, 1977, we plan to particlpate in high energy physics experiments at the Fermi

National Accelerator Laboratory (FNAL), at the Argonne Zero-Gradient Synchrotron (ZGS), and at CERN Super Proton Synchrotron (SPS). We will also complete an analysis of an experiment performed at SLAC and will undertake development work for a possible experimental program at PEP. In one of the FNAL experiments, the polarization of recoil protons from elastic p-p scattering will be measured for $20 \mathrm{GeV} / \mathrm{c}<$ $P_{\text {LAB }}<400 \mathrm{GeV} / \mathrm{c}$ for $|t|$ between 0.2 and $2.0(\mathrm{GeV} / \mathrm{c})^{2}$. This experiment is being done in the internal target area and will use a gas jet in conjunction with a recoil proton spectrometer and a carbon analyzer. In the other Fermilab experiment a Caltech-FNAL-UICCIndiana-UCLA group will use a multiparticle spectrometer to make a global study of reactions in which one of the final state particles or group of particles carries off large transverse momentum. In the ZGS experiment, the polarized beam and a carbon polarimeter will be used to measure the polarization, depolarization, and asyumetry parameter in inclusive p-p scattering. A Saclay-Indiana group will conduct a charmed particle search at the CERN SPS. This experiment will utilize a vertex spectrometer to look at the decay products of charmed particles triggered by the presence of two $\mu$. Thus the $2 \mu$ decay of the $\Psi$ will be used to enrich the charmed particle component of our data. The analysis of the SLAC experiment, an IndianaVanderbilt-Purdue-SIAC effort, is nearly complete. An automatic film measuring system (PEPR) is being used to analyze streamer chamber film from this experiment which is a search for manifestly exotic mesons with double charge created in $\pi^{-} d \rightarrow \hat{p}_{\text {fwd }} \mathrm{p}_{s} \mathrm{x}^{--}$.

Goals and/or Major Results to Date

The AEC/ERDA-sponsored Indiana high energy physics group has performed a series of experiments at the Argonne ZGS, measuring elastic scattering cross sections, determining various properties of $\mathrm{A}_{2}$ meson production and decay, and investigating spin effects in high energy scattering. Currently we are active in two Fermilab experiments and are also involved in efforts at SLAC (exotic meson search) and the 


\title{
INDIANA UNIVERSITY ...:
}

\author{
Bloomington, Indiana
}

CERN SPS (charm search). The Fermilab work is being done on multiparticle production processes and pp $\rightarrow$. pp polarization. Since its inception on December 1, 1968, the group has published 11 articles in Physical Review, 6 in Physical, Review Letters,: 2 in Physics Letters 2 in Nuclear Physics, and 2 in Nuclear Instruments and Methods.

$$
* \dot{*} \dot{*} * \dot{*} * * * * * * * *
$$

Title of Project: Theoretical Particle Physics

Person in Charge: A. Hendry, D. Lichţenberg, D. Weingarten

Sclentific and Professional staff: A. Hendry, D. Lichtenberg, D. Weingarten

\section{Scope of Work}

Our proposed program contains a balance of studies involving hadron collisions and investigations of quark models incorporating charm. In the first category, a complete partial wave analysis of pionnucleon scattering up to $10 \mathrm{GeV} / \mathrm{c}$ is proposed, in order to extract information about high mass, high spin resonances; a cascade model for multiparticle production will also be developed. As regards quark models, we intend to consider, various schemes which will produce the observed spectrum of states, including charmed particles, as well as a study of their production in collisions. Continued collaboration with the Indiana University experimental group (Task $; A)$ is anticipated as several of their experiments come to completion this year and new ones are developed.

Goals and/or Major Results to Date.

We have previously obtained evidence for several new high mass, high spin $\Delta$ resonances in pion-nucleon scattering, and examined high energy collistions in the framework of Reggeon field theory. We have also considered various quark model schemes to obtain the general spectrum of mesons (including charmed mesons). Further topics of study have been electromagnetic mass splitting among the mesons, the pion form factor, and the magnetic moments of baryons.

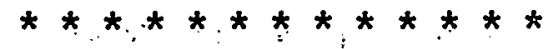




\section{INSTITUTE FOR ADVANCED STUDY \\ Princeton, New Jersey}

Contract No. EY-76-S-02-2220

Title of Project: Problems in Particle Theory

Person in Charge: S. Adler, R. Dashen

Sclentific and Professional Staff: T. Banks, H. Duncan, R. Gonsalves, A. Jevick1, S. Joglekar, J. Koplik, Y. Ne'eman, S. Nussinov, N. Papanicolaou, J. Rosner, S. Shei, M. Virasoro, C. Callan

\section{Scope of Work}

This contract supports the research, in high energy theory, of a number of members of the Institute for Advanced Study. For a thcoretical one the group is large and essentially all areas of particle theory are represented. This leads to considerable cross fertilization and provides an atmosphere which is advantageous to the development of young physicists. However at a given time certain areas tend to be emphasized. At present the main effort is directed towards a particular fleld theory, quantum chromodynamics, which is generally believed to underlie the interactions of quarks and their binding into hadrons. This theory has had some qualitative success but because of one's Inability to calculate has not been quantitative. Much effort 18 going into attempts to make the theory quantitative. There is also considerable work on the phenomenology of weak interactions and charmed particles.

\section{Goals and/or Major Results to Date}

Recent work has lead to new Insights into field theory in general and quantum chromodynamics in particular. The problem of the "ninth Goldstone boson" has been solved and problems assoclated with the spontaneous breakdown of chiral symmetry have been clarified. Some new ideas about quark confinement appear to be emerging.

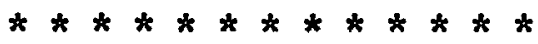


JOHNS HOPKINS UNIVERSITY.

Baltimore, Maryland

Contract No.

$E Y-76-S-05-3285$

Title of Project: Research in Theoretical Physics

Person in Charge: G. Domokos.

Scientific and Professional Staff: G. Domokos, S. Koves1-Domokos

Scope of Work

Develop algebraic approach to quark problem. Despite numerous at tempts at "dynamical" confinement of quarks, no entirely satisfactory theory was developed along this line. We suspect that the existence of extra quantum numbers which are exactly conserved (Ge11-Mann's "color") leads to unfamiliar algebraic properties of quarks as dynamical coordinates, thus making quarks unobservable as free particles. The scope of the research is to construct such algebraic models in terms of quark variables which i) retain the main desirable features of present-day "naive" composite models of hadrons, $i i)$ prevent the appearance of free quarks as a consequence of the algebraic relations obeyed by the quark variables. Our approach consists of a generalization of the ideas of Gursey and his collaborators. These authors observed that certain mathematical structures known in abstract algebra (Hurwitz and Rozenfeld algebras) exhibit triality properties which are analogous to the color selection rules obeyed by quarks. We wish to develop a quantum theory of quarks along these lines. This requires a generalization of ordinary Fermi and Bose statistics to an unusual "quark-statistics." If successful, such an approach would describe the substructure of strongly interacting particles in terms of a: generalized "exclusion principle," in the same way as the Pauli exclusion principle characterizes the well-known shell structure of atoms and nuclei.

Goals and/or Major Results to Date

We succeeded in constructing a simple model in which i) quarks obey physically meaningful "color selection rules" exactly, ii) leptons and quarks behave in a similar fashion. (The existence of such a model is important in that it demonstrates the feasibility of our approach, which by itself is a non-trivial question.) The present model under study--though obviously oversimplified--is already capable of predicting certain features of the particle spectrum (e.g. it predicts the existence of heavy leptons), thus it is experimentally testable. Present investigations concentrate

- upon further development of the model so as to include space-time 


\section{JOHNS HOPKINS UNIVERSITY}

Baltimore, Maryland

properties. This will allow us to make at least semi-quantitative predictions of mass ratios, decay rates and to establish--approximate--selection rules, thus broadening the range of testable predictions.

$$
\star * * * * * * * * * * * *
$$




\section{LEHIGH UNIVERSITY \\ Bethlehem; Pennsylvania}

Contract No.

EY-76-S-02-2894

Title of Project: . Study of Multiparticle Jet Production UsIng Calorimeters

Person in Charge: A. Kanofsky

Sclentific and Professional Staff: A. Kanofsky, G. Lazo, R. Wolfinger,

D. Allen, A. Hasan, D. Gockley, F. Klenk

Scope of Work

The scope of the Fermilab work is to obtain information on Jets resulting from direct quark-quark collisions. An experiment was performed at the Argonne Streamer Chamber facility (ANL experiment \#406) to study the interactions of particles ( $\left.\pi^{\prime} s, p^{\prime} s\right)$ with nuclei (Ne and $\mathrm{He}$ ). We have measured cross sections of hypernuclel ( $A^{*}$ ) formation and decay into a Gamma Ray at the AGS (Experiment \#664) using the reaction $K^{-}+A \rightarrow A^{*}+\pi^{-}, A^{*} \rightarrow \gamma+A$ where the $\gamma$ ray and pion of the final state are detected. Experiments have been performed in the AGS test beams to study the channeling of particles of $\mathrm{GeV} / \mathrm{c}$ momenta. Using a simple three quark (proton) and two quark (pion) Glauber scattering model, we have been able to explain the changing slope at low momentum transfer and the rise in multiplicity with increasing momentum transfer to the leading particle. Work is continuing on an experiment to search for coincidences between extenstve air showers and Weber pulses.

Goals and/or Major Results to Date

Results on the Lambda Nucleon Spin-Spin coupling, multiple quark scattering in pp elastic scattering, and the performance of a large streamer chamber in extensive air showers.

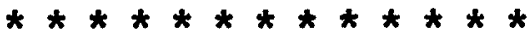


UNIVERSITY OF MARYLAND

College Park, Maryland

Contract No. EY-76-C-05-2504

Title of Project: High Energy Accelerator and Colliding Beam User Group

Person in Charge: G. A. Snow

Scientific and Professional Staff: R. G. Glasser, B. Sechi-Zorn,

G. A. Snow, P. H. Steinberg, G. B. Yodh, G. T. Zorn, C. Y. Chang;

T. Dombcck, G. Meclellan, L. H. Junes, M. Kazuno, L. I'e1g, A. Skuja

Scope of Work

The aims of this high energy physics, resparch program are to devioc and carry out experiments that will uncover and elucidate the properties of "elementary particles" and their interactions. Strong, electromagnetic, and weak interactions are studied using many techniques including counters, spark chambers, and bubble chambers.

In strong interactions, the program includes: analysis of pp and $\pi \mathrm{p}$ collisions from 100 to $360 \mathrm{GeV} / \mathrm{c}$ using bubble chamber hybrid system or alternatively the $15^{\prime}$ bubble chamber at Fermilab; a high statistics $\mathrm{K}_{\mathrm{p}}^{+}$inelastic cross section experiment at $1.6-1.9 \mathrm{GeV} / \mathrm{c}$.

In electromagnetic interactions, analysis of a SPEAR $e^{+} e^{-}$experiment at the $\Psi, \Psi^{\prime}, 4 \mathrm{GeV}$ and $6-7 \mathrm{GeV}$ regions with emphasis on $\gamma_{\mp}$ ray
emission and inclusive muon and hadron spectra; study of $e^{2}$ collisions at 1-2 GeV with MEA at ADONE; design and development of a detector facility for PEP.

In weak interactions, high energy neutrino interactions with deuterium at Fermilab with spectal emphasis on search for charmed baryons; search for $\left(\mu^{+} e^{-}\right) \rightarrow\left(\mu^{-} e^{+}\right)$transitions at SREL, study of exotic neutrino reactions at Fermilab.

Goals and/or Major Results to Date

Recent contributions have included; evidence for annmalous, inclusive muon production in $e^{+} e^{-}$collisions at $4.8 \mathrm{GeV}$ and for discrete $\gamma$-ray lines from $\Psi(3.7)$ decays, measurement of cross sections, mult1plicities and correlations in $\pi^{-} p$ collisions at the highest avallable energy $(360 \mathrm{GeV} / \mathrm{c})$, characteristics of strange particle production in $100 \mathrm{GeV} / \mathrm{c} \pi \mathrm{p}$ collisions, properties of $\equiv$ resonances and $\phi$ decayo, improvements in widely used bubble chamber analysis programs and in the Md. PEPR, phenomenology of heavy lepton and charm particle decays.

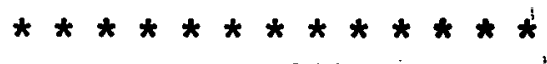




\section{UNIVERSITY OF MASSACHUSETTS \\ Amherst, Mass achusetts}

Contract No. . EY-76-S-02-3330

Title of Project: High. Energy Physics

Person in Charge: J. Button-Shafer

Scientific and Professional Staff: R. Lichti, S. Dhar, L. Latour

Scope of Work

University of Massachusetts has constructed target-region apparatus for the Brookhaven Multi-Particle Spectrometer (MPS), a large

Cerenkov counter and an array of time-of-flight counters; assistance has been given also in the construction and testing of target-region spark chambers. Some of this apparatus has been used for a search for the spinless "paracharmonium" state and also for pecent run to investigate high-mass strange resonances called $\mathrm{K} s$. New or modified equipment is being readied for a "charm" experiment at the MPS which will study "associated production" of charmed objects decaying into "hadrons." Considerable effort has gone into the successful development of a polarized target which will be used at the MPS, or:possibly at SLAC's LASS. Interest is developing in PEP.

Goals and/or Major Results to Date

Polarization achieved with the University of Massachusetts polarized target is $\gtrsim 65 \%$ at $10.5 \mathrm{kG}$ and $1.3^{\circ} \mathrm{K}$; and $75-80 \%$ polarization is expected to be attainable with a $15 \mathrm{~kg}$ field. Prospects for use in. studying strange-particle processes at the MPS look good.

Data obtained with the MPS in the region of the $\mathrm{K}^{*}(1780)$ exceed bubble-chamber data by at least an order of magnitude. Analysis is nearly finished. Testing for the MPS experiment (charmed decay into hadrons) went very well; the full-scale run is planned for June 1977. 
UNIVERSITY OF MINNESOTA

Minneapolis, Minnesota

Contract No. EY-76-C-02-1764

Title of Project: High Energy Physics

Person in Charge: S. Gasiorowicz

Scientific and Professional Staff: S. Gasiorowicz, D. Geffen,

J. Rosner, H. Suura, Y. Tang, B. Bayman, H. Courant, K. Ruddick,

P. Ellis, W. Wilson, S. Tsai, E. Peterson, M. Marshak, E. Marquit

Scope of Work

1) Theoretical. studies in quark confinement: Work is being carried out on classical solutions to field equations derived from Yang-Mills Lagrangians. The quantum problem of confinement is being studied in the framework of Wilson's lattice gauge theory.

2) Charmed particle phenomenology: Detailed studies of production and decays of charmed particles, and charmonium states are being. carried out. The whole array of tools of the trade, e.g., Reggeology, the quark model and current algebra is being applied to this problem.

3) Diffraction Scattering at very high energies: A detailed analysis of the implications of new high energy data for the ChouYang model and its generalizations is being carried out.

4) Studies in nuclear structure and nuclear reactions: Studies of bound and continuum states of nuclei, effective interactions between nucleons; and the mechanisms of reactions involving complex nuclet.

Goals and/or Major Results to Date

1) The goals here are to see how the classical structure of the equations forces local "lump-like" solutions, and what kind of effective potentials are forced by the gauge theory and the selfcouplings. Furthermore the similarities and differences between lattice and continum theories need to be explored.

2) The purpose is to suggest we11-defined experiments to test present theories of the charmed particles and answer questions posed by the "old"' theories involving only the conventional particles.

3) The goal is to check the validity of the Chou-Yang model, the usefulness of the eikonal expansion and the dependence of fine details of the data on assumed form factors for the nucleon. 


\section{UNIVERSITY OF MINNESOTA. \\ Minneapolis, Minnesota}

4) To determine the extent to which observed properties of nuclei can be understood in terms of a system of interacting nucleons, and to explore the consequences of dynamical models used in the description of nuclear excitations and reactions.

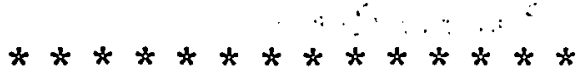


NORTHWESTERN UNIVERSITY

Evanston, Illinois

Contract No. EY-76-C-02-2289

Title of Project: High Energy Experimental Physics

Person in Charge: D. Miller, J. Rosen

Scientific and Professional Staff: M. Block, B. Gobbi, J. Keren,

D. Buchholz, R. Ruchti, T. Phillips, R. Tilden, D. Spelbring,

R. Lipton, K. Nishikawa, R. Lord, L. Kenah

Scope of Work

The N.U. group is participating in a variety of high energy experiments using the counter chamher technique to providc clectronically recorded data. Four projects are underway.

1) The NU.-Rochester-Fermllab group (Rosen, Gobb1, Keren) has recently completed E-397--search for associated hadronic charm production. The search for explicit charm decay final states is keyed by triggering on prompt muon production provided by the decay of the companion charm particle. The $\mu \mu$, $\mu e$, and $\mu-K \pi$ events have been studied. A new proposal, P-515 (N.U.-Carnegie MellonFermilab) has been submitted. The search will employ a new powerful spectrometer system, utilize incidence pions and will be 100 fold more sensitive.

2) Block is participating in the CHORMN collaboration at ISR on a similarly motivated series of experiments which feature a prompt electron signal. The inclusive spectra of prompt electrons with low $p_{1} \cdot(0.3-1.0 \mathrm{GeV} / \mathrm{c})$ and $p(0.6-2.0 \mathrm{GeV} / \mathrm{c})$ has been reported.

3) Buchholz is working with the Cal Tech group on neutrino experiments at Fermilab employing a narrow band $v(\bar{v})$ beam and a large scintillator-chamber-Fe toroid spectrometer.

4) Miller and Gobbi are working with SLAC-LBL-Hawa11 on $\mathrm{e}^{+} e^{-}$ annihilation physics at SPEAR. The experiment features a $\mathrm{Pb}$ glass wall system for electron and $\gamma$ ray detection which supplements the capabilities of the presently employed magnetic spectrometer.

Goals and/or Major Results to Date

E-397 preliminary results place an improved upper limit on hadronic charm production of $20 \mu \mathrm{b}$. The same group has reported comprehensive results on neutron diffractive and coulombic dissociation providing detalled information on the Deck model and resonance production. 


\section{NORTHWESTERN UNIVERSITY \\ Evanston, Illinois}

The ISR group has observed significant increase in $e / \pi$ for low $p$ and $\mathrm{p}_{\perp}$ prompt electrons as contrasted with previous measurements at large $p$. Interestingly, this is the regime in which charm semileptonic decays are expected to contribute, although other production sources cannot be ruled out as yet.

The neutrino group has measured the structure of charged and neutral currents, observed events with two and three final state muons and set new lower limits on the masses of intermediate vector bosons and heavy leptons.

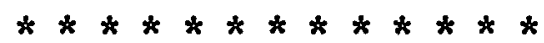


OHIO STATE UNIVERSITY

Columbus, Ohio.

Contract No. . EY-76-C-02-1545

Title of Project: High,Energy Physics (Experimental)

Person in. Charge: T. Romanowski

Scientific and Professional Staff: T. Romanowski, K. Reibel, N. Reay, N. Stanton, A. Lesnik, J. Fitch, J.. Heimaster, C. Rush

Scope of Work

Our effort in experimental high energy physics is concentrated at two laboratories; the Fermilab and the ZGS at Argonne.

The Fermilab experiment on: the search for charmed particles (E-366 Fermilab) has been recently completed and the final data analysis is in progress. Analysis of an experiment on the neutron-proton charge exchange between 40 and $300 \mathrm{GeV} / \mathrm{c}(E-12$ Fermilab) has been completed. At present we are formulating our new research goals for Fermilab.

Experiments at the ZGS which we are pursuing now are: A study of meson spectroscopy in the mass region. $0.1-2.0 \mathrm{GeV} / \mathrm{c}^{2}$. with the Charged-Neutral Mass Spectrometer.. Analysis of the data previously taken with that apparatus on $\omega$ production (E-37.7 ZGS), $\rho-\omega$ interference (E-380 ZGS) is proceeding. Search for new resonances $A_{1}{ }^{\circ}$, $\mathrm{H}, \mathrm{D}, \delta,(\mathrm{E}-397 \mathrm{ZGS})$ is also in progress. An experiment to study neutral meson decays into $\pi^{+} \pi^{-} \cdot 3 \gamma$ and $\pi^{+} \pi^{-} 4 \delta$ has been proposed and is approved ( $E-420 \mathrm{ZGS})$; also an experiment to study the leptonic decays of polarized $\Sigma^{-}$hyperons ( $E-347$ ZGS) is in progress to test the Cabbibo model for the leptonic decays of strange particles. Development of the drift chamber technology is continuing.

Goals and/or Major Results to Date

The neutron-proton charge exchange measured at Fermilab in the E-12 experiment yielded a surprising evidence of pion exchange dominance. The charm search E-366 will set a limit on the production with neutrons of the; narrow dihadron states in the mase range between $1.8-4.0 \mathrm{GeV} / \mathrm{c}^{2}$. The experiments on the meson spectroscopy at the:ZGS with the Charge-Neutral Spectrometer study production and determine quantum numbers which describe these. resonances. The unique capability of this spectrometer is that it can detect $\pi^{\circ}$ and $\gamma$ rays with high efficiencies which.makes it particularly suitable to study the meson resonances decaying into neutral states composed of charged and neutral particles. 


\section{OHIO STATE UNIVERSITY \\ Columbus, Ohio}

The aim of the experiment on the $\Sigma^{-} \beta$ decay is to measure sign of the ratio of axial to vector form factors which was predicted by Cabbibo model. The knowledge of the character of the weak interaction in the leptonic decays of strange particles is an important input to the gauge theory which unifies the weak and electromagnetic interactions.

$$
\text { **************** }
$$

Title of Project: High Energy Physics (Theoretical)

Person in Charge: K. Tanaka, W. Wada

Scientific and Professional Staff: K. Tanaka, W. Wada, W. Palmer,

S. Pinsky, E. Takasugi, J, Hietarint;

Scope of Work

The main topics of the theoretical investigation will remain to be on symmetry considerations and dynamical calculations. Under the former category are deep inelastic data as a test of hadron symmetries, spontaneous breakdown of chiral and gauge symmetry, invariance under Abelian, non-Abelian and supersymmetry transformations, low energy theorems, and $S U(3)$ and $S U(4)$ symmetry related to the decay of new resonances.: Under the latter category are production and decay of new resonances, violation of Zweig rule, Reggeon fleld theory, parton fragmentation model phenomenological bounds in $\nu$ interaction and dimuons, mixing models, solution to non-linear field theoretic models, ue production, and level shift and charm mass. Study of quark models with several flavors will also be pursued.

\section{Goals and/or Major Results to Date}

Study the phenomenology of narrow resonances and neutrino interactions, non-linear field theories and quark models, charm meson production. and charmonium type models, and quantum chromodynamics and instanton interaction.

Results--September 30, 1975 to present: Under symmetry considerations obtained tests of hadron symmetries from deep inelastic data, low energy theorems in supersymmetry, SU(3) and SU(4) symmetry related to the decay of new resonances; and under dynamical considerations calculated production and decay of new resonances, quantum effects. of instanton solutions, effects on non-diagonal terms in the neutral current in a six quark model, and charm meson production in electronpositron annihilation.

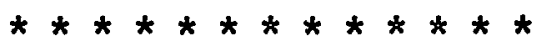


OKLAHOMA STATE UNIVERSITY

Stillwater, Oklahoma.

Contract No. EY-76-S-05-5074

Title of Project: Higher-Order Corrections to the Anomalous Magnetic Moment of the Muon

Person in Charge: M. Samuel

Scientific and Professional Staff: C. Chlouber

Scope of Work

A calculation of the contrihution of a ccrtain slass of Feyñman diagrams to the muon anomalous magnetic moment is underway. This class contains the 18 diagrams obtained by inserting a single electron loop in all possible ways in the photon-photon scattering sixthorder graphs and provides the dominant contribution in eighth-order. The calculation will make use of the techniques of Padé approximants to accelerate convergence of the sequences obtained from the multidimensional numerical integrations.

As well, more accurate values for the sixth-order contributions will be obtained. In some cases, this will involve reducing numerical integration errors, again using Pade approximants to accelerate convergence, for others, it will involve obtaining corrections of order $\mathrm{m}_{\mathrm{e}} / \mathrm{m}_{\mu}$, which have not yet been calculated. This will increase the accuracy of the quantum electrodynamical prediction necessary to keep pace with the on-golng effort to improve the experimental value.

The new $(g-2)$ muon experiments, now underway at CERN, which may achieve a precision of $10 \mathrm{ppm}$, as well as future, presumably even more accurate experiments, demand an improvement, on the theoretical side, in the knowledge of the pure QED contributions. The comparison between theory and experiment will provide an extra-ordinarily sensitive probe of possible new interactions, as well as any muon substructure.

Goals and/or Major Results to Date

The sixth-order phnton-photon arattering concribution has been evaluated; the result is $\Delta \mathrm{a}_{\mathrm{ph}-\mathrm{ph}}=(21.32 \pm 0.05)\left(\frac{\alpha}{\pi}\right)^{3}$. See "Photon-Photon Scattering Contríbution to the Anomalous Magnetic Moment of the Muon," Physical Review Letters, 36, 442 (1976). Computations are presently being done to accurately determine this contribution for the clectron. 


\section{OKLAHOMA STATE UNIVERSITY}

Stiliwater, Oklahoma

The calculation of the eighth-order contribution, referred to, above is well underway, Here we are also independently determining the coefficients of $\ln ^{2} \mathrm{~m}_{\mu} / \mathrm{m}_{e}$ and $\ln \mathrm{m}_{\mu} / \mathrm{m}_{e}$. which occur in the result.

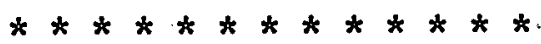




\section{UNIVERSITY OF OREGON \\ Eugene, Oregon}

Contract No. EY-76-S-06-2230

Title of Project: Theory of Elementary Particles

Person in Charge: M. Moravcsik, P. Csonka, R. Hwa

Scientific and Professional Staff: M. Moravcsik, P. Csonka, R. Hwa

$\underline{\text { Scope of Work }}$

1) Solution of the sourceless Yang-Mills equation.

2) Application of the color gauge group to the theory

of asymptotic freedom.

3) Calculations in SU(4) symmetry with an eye toward new particles.

4) Psion cross sections in multiperipheral models.

5) The connection between scaling violation and large $p_{T}$ reactions.

6) Renormalization of pomerons.

7) Higgs bosons in $\mathrm{SU}(2) \times U(1)$ gauge theory.

8) New variants of weak interaction theories.

9) X-ray lasers.

10) X-ray holography.

11) Determination of reaction amplitudes from polarization experiments.

12) Polarization experiments as a tool to study off-shell effects.

13) Polarization experiments as a dynamical probe of particlc reaclions.

14) An overall analysis of elementary particle physics, its rate of progress, and the measures that could be taken to enhance this rate. 


\section{UNI VERSITY OF OREGON \\ Eugene; Oregon}

Goals and/or Major Results to Date

1) Considerable theoretical advances in X-ray holography and laser accelerators.

2) New insights intó the connection between scaling violation, extended structure of quarks, and large momentum transfer reactións

3) A cleaner way of studying off-shel1 effects in five-particle reactions through the observation of polarization quantities which vanish on-she11 because of symmetry constraints.

4) A coherent though in part speculative analysis of the status of particle physics and a set of specific suggestions to acce1erate its progress.

$$
* * * * * * * * * * * * *
$$

$$
\because \cdots \cdots
$$
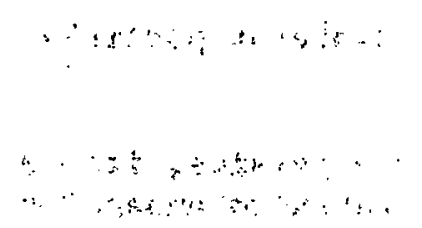


\section{PHYSICS INTERNATIONAL COMPANY}

San Leandro, California

Contract No: $\quad$ EY-76-C-03-0948

Title of Project: Linear Collective Acceleration of Protons and Ions by Intense Electron Beams

Person in Charge: S. Putnam

Scientific and Professional Staff: B. Ecker

$\underline{\text { Scope of Work }}$

This program is an experimental study of collective particle acceleration by intense pulsed electron beam injection into initially neutral gas. Beams of typically $100 \mathrm{kA}, 1 \mathrm{MeV}, 50 \mathrm{~ns}$ duration and 8 ns current risetime are injected into neutral hydrogen in the $0.1-0.7$ torr pressure range. Experiments consist of examining the dependence of accelerated proton and beam front velocity on beam parameters, hydrogen density, and system geometry to determine the governing physics and the eventual range of particle energies attainable.

\section{Goals and/or Major Results to Date}

Compact bunches of $10^{11}-10^{13}$ protons have been accelerated to $15 \mathrm{MeV}$ using $1 \mathrm{MeV}$ electron beams with accelerating fields on the order of $10^{6} \mathrm{~V} / \mathrm{cm}$. Proton velocity equals beam front velocity, and parameter variation data indicate that beam front velocity is governed by a power balance in which the kinetic energy of the electron beam is expended in self-field generation at the propagating beam front. The results appear inconsistent with the model proposed by 01son. Present focus is on the implications of the power balance model for considerably increased proton energies using proper tailoring of beam, gas, and geometry parameters.

$$
\star \star \star \star \star \star * \star * * * * * * *
$$


PURDUE UNIVERSITY

Lafayette, Indiana

Contract No.

$E Y-76-C-02-1428$

Title of Project: Experimental High Energy Physics Program

Person in Charge: F. Loeffler

Scientific and Professional Staff: J. Gaidos, F. Loeffler, R. McIlwain, D. Miller, T. Palfrey, Jr., E. Shibata, K. Stanfield, R. Willmann, I. Rangan, R. De Bonte, W. Ditzler, C. Wilson

Scope of Work

Our group at Purdue is a rather large and diverse one as university high energy physics faculties go. We are studying strong interactions among elementary particles using triggered bubble chamber as well as counter-electronic techniques. One of our main interests has been a study of the systematics of meson and baryon resonances--often called hadron spectroscopy. We are currently carrying out experiments at SIAC, Argonne, and Fermilab and are very much in the race to discover "charmed" hadrons by using a two-arm spectrometer in a $400 \mathrm{GeV}$ proton beam at the Fermilab Meson Area.

Another important research program involves use of the SLAC 40" triggered bubble chamber in a search for rare decays of meson states. In particular, we are selecting on $\mathrm{k} \bar{K}$ and $\bar{p} \bar{p}$ final states. Our POLLY system is fully operational and has been in production for several months with this film.

A unique and interesting experiment is just now getting underway with an internal gas jet target in the main ring (C-0 section) at Fermilab. Various heavy gases will be used in a study of heavy fragment production $(4 \leq z \leq 16)$ in high energy proton-nucleus collisions to learn about collective motion induced by fast protons as they pass through nuclei.

Goals and/or Major Results to Date

Our main goals at the present time are to help determine the details of the spectra of strongly interacting particles. As has been often and truly said, we can't hope to understand the "new" particles until we understand the symmetries governing the "old." In the past few years results from our group have shed considerable light on meson and baryon resonances. In fact, one of our experiments in 1971 led to the discovery of a new state, the $\mathrm{K}^{*}(1760)$. At the higher energies afforded by Fermilab we expect to continue this work, for example, by systematic searches for charmed hadrons and studies of their properties.

$$
* * * * * * * * * * * * *
$$


PURDUE UNIVERSITY

Lafayette, Indiana

Title of Project: Theoretical Study of E.lementary Particles

Person in Charge: M. Sugawara

Scientific and Professional Staff: R. Capps, P. Rosen, A. Tubis,

L. Balázs, E. Fischbach, N. Fuchs, : T. Kưo, B. War̀d: $: \because ; \ldots$

Scope of Work

$\therefore$

Our theoretical studies of elementary particles comprise such a variety of subjects that some are quite basic but others are to be regarded as phenomenological.

Basic studies include the study of Sugawara on the formulation of gauge theory of elementary particles without including the so-called charmed elementary particles, and the study of these charmed particles by Kuo in which the production of $\mu$-pairs, in particular, is analyzed to find an evidence for or against the charmed particles." Also included in the basic studies is the stuidy of broken symmetries by Fuchs which aims at finding a systematic way to handle broken symmetries of elementary particles, and the stidy of the new type of bound states by Ward, in which the binding mechanism is: not hitherto known but due to Ward himself, which aims at explaining the newly discovered very narrow particles........

Phenomenological studies include the study of the structure of weak interactions by Rosen and Fischbach in which the current main interest lies on the structure of the neutral weak current, and the study of Capps in which he explores the consequences of various internal symmetries of elementary particles. Also :included in the phenomenological studies is the study of Balázs on the production. of many particles in terms of Regge-pole theory; and the study of Tubis on those properties of a few nucleon systems which are relevant to elementary particle physics.

Goals and/or Major Results to Date

Presumably the most significant result of the studies outlined above is the finding that, even if no charmed particles exist; the gainge theory of elementary particles can be formulated successfully but implies the existence of very heavy particles $(200 \sim 300 \mathrm{GeV}$ in mass) which are also responsible to weak interactions:.

Other significant results are that the $\mu$-pair experiments can be explained in terms of charmed vector mesons and that the neutral. weak current appears to have the same structure as the charged one.

$$
* * * * * * * * * * * \dot{*} * *
$$


PURDUE UNIVERSITY

Lafayette, Indiana

Title of Project: Research on Nucleon Structure and Sub-Nucleonic Components

Person in Charge: E. Fowler

Scientific and Professional Staff: N. Baggett, D. Hood

Scope of Work

The objective of this, experimental research is to learn more about the structure and nature of the subnuclear particles. Much has been done in the field and it is clear that most of the so-called "elementary" particles possess a structure. The nature of such structure is not clear. With regard to hadrons and the possibility of their being "made of" quarks, we have been concentrating on a search for "exotic mesons" whose very existence would rule out the simple quark model. We have achieved an important first step in a hybrid bubble chamber experiment (SLAC E-82), and are well along in an effort to use the SLAC Streamer Chamber to probe considerably deeper in that search. The data-taking for SLAC E-103 has been completed. In addition, we are engaged in making measurement of the cross section of the inverse $\mathrm{K}$-capture of $\mathrm{Ar}^{37}$, namely, $v_{e}+\mathrm{Cl}^{37} \rightarrow \mathrm{Ar}^{37}+\mathrm{e}^{-}$, using the $\nu_{e}$ source provided by the Los Alamos Meson Physics Facility. Preliminary testing is underway. The experiment, whose result is of considerable importance to interpreting the BNL Solar Neutrino Experiment, cannot be done before mid-1978. Final1y, we expect to be able to carry out a high energy neutrino experiment in collaboration with the Cal TechFNAL group of Experiment 21, to test the identity of the neutral. lepton from $\pi$-decay to that from $K$-decay. In a number of the ways that have been put forth to combine the laws concerning strong, weak, and electromagnetic interactions, it turns out that one would prefer that the decay neutrino of the $\mathrm{k}$-meson i.s different from that of the $\pi$-meson. No definitive experimental test of that hypothesis has been made. Our first test will be to compare their cross section (in iron) at the same energy. We are also seeking to develop a new kind of vertex detector with liquid hydrogen.

\section{Goals and/or Major Results to Date}

Results are reported from a hybrid experiment designed to search for the backwards-produced charge $=+2$ exotic meson resonances by investigating the reaction $\mathrm{f}^{+}+\mathrm{p} \Rightarrow \mathrm{X}^{++}+\mathrm{n}$ (forward) at $8.4 \mathrm{GeV} / \mathrm{c}$. From a theoretical point of view, the backwards-production reaction is: a favored mode of exotic-meson production if two-component duality arguments are meaningful. The data reported here is at a statistical level corresponding to approximately 150 events per 
PURDUE UNIVERSITY

Lafayette, Indiana

microbarn. No clear evidence for narrow exotic mesons has been found in this experiment. In our FNAL experiment the velocity of $80 \mathrm{GeV}$ neutrinos has been measured by time of flight to be the same as that of light in vacuo to about 3 parts in $10^{4}$.

$$
* * * * * * * * * * * * * *
$$

Title of Project: An Experimental High Energy Physics Program

Person in Chargc: V. Barnes, I1, D. Carmony, A. Garfinke1, L. Gutay

Scientific and Professional Staff: V. Barnes, II, D. Carmony, R. Christian, A. Garfinke1, L. Gutay, R. Scharenberg, L. Rangan, R. DeBonte, A. Laasanen, C. Wilson

Scope of Work

Our research in experimental particle physics presently involves neutrino physics at both Fermi National Accelerator Laboratory (FNAL) and Argonne National Laboratory (ANL) and hadron physics at FNAL. The neutrino program at FNAL (E-31) involves studying the interactions of a high energy, broad band, antineutrino beam with protons in the FNAL 15-foot bubble chamber. It utilizes the External Muon Identifier. At ANL it involves, studying the interactions of a low energy neutrino beam with protons and neutrons in the deuterium fitted 12-foot bubble chamber. One branch of the hadron physics program is using purely electronic techniques in the FNAL CO Area to study the interactions of protons with nuclei. The other branch utilizes the 30 -inch bubble chamber and associated detectors to study the interactions of high energy hadrons with protons. In the past these hadrons have been $100 \mathrm{GeV} \pi^{+}$and $\mathrm{K}^{+}$ mesons as we11 as protons. Continued research in all of these programs is planned.

Goals and/or Major Results to Date

One goal of the neutrino program is to understand the weak neutral current and to learn if a unified theory of weak and electromagnetic interaction is possible. We rontributed to the dlscovery of tie neulral current and have recently reported results on its isotopic spin structure. One goal of the hadron program is to find evidence of the underlying quark structure of hadronic matter. We found some such evidence in aur studies of $\mathrm{K}^{+} \mathrm{p}$ interactions at $100 \mathrm{GeV}$. Another goal of the hadron prngram is to otudy the 1nteresting phenomenon of "shock waves" in nuclear matter.

$$
* * * * * * * * * * * * * *
$$


RICE UNIVERS ITY

Houston, Texas

Contract No. EY-76-S-05-5096

Title of Project: High Energy Physics

Person in Charge: G. Phillips, J. Roberts, Jr.

Scientific and Professional Staff: G. Phillips, J. Roberts, Jr., J. Buchanan, Sr., J. Clement, W. Madigan, G. Mutchler, J.

Gabitzsch

\section{Scope of Work}

We are engaged in a program of studying the spin dependence of the nucleon-nucleon interaction in the energy range 1 to $12 \mathrm{GeV} / \mathrm{c}$ at the Argonne Zero Gradient Synchrotron. The experiments include one-spin and two-spin measurements for proton-proton scattering (and both elastic and inelastic $p-p$ interactions), and one spin measurements for proton-neutron elastic scattering. Experiments completed or scheduled for the near future include ZGS E-393, E-395, E-407, E-408, and E-418. In these experiments, either done in collaboration with the University of Minnesota, or manned largely by Rice with University of Houston and University of Michigan collaborators, we have measured the asymmetry and depolarization in the inclusive processes $p+p \rightarrow \pi \pm, K^{ \pm}, p, d,+$ anything at 6 and $12 \mathrm{GeV} / \mathrm{c}$; we have measured the spin dependence of the total and small angle elastic p-p cross sections from $1-3 \mathrm{GeV} / \mathrm{c}$, and have measured the polarization and depolarization in neutronproton elastic scattering at $6 \mathrm{GeV} / \mathrm{c}$. We will measure the $\mathrm{n}-\mathrm{p}$ elastic polarization out to very large angles in the near future. We are also engaged in a collaboration with Stanford and Fermilab (Fermilab E-192 and E-193) in experiments to study multi-gamma ray final states in $\pi^{-} p$ and to study deep inelastic electron scattering at Fermilab, and in ZGS $E-415$, an Argonne-ColumbiaMinnesota-Rice collaboration to study direct electron production from 2-12 GeV/c.

\section{Goals and/or Major Results to Date}

Our goal is to engage in a program of measurements which will help lead toward an understanding of the dynamics of the interactions of elementary particles: the measurement. of the spin dependence of elastic and inelastic nucleon-nucleon scattering is essential to an understanding of the strong interaction; measurements such as the direct electron production from intermediate to high energies may shed light on the dynamics of strong, weak, and electromagnetic interactions. 
RICE UNIVERSITY

Houston, Texas

Some of our major results to date are the following: There are large asymmetries $(\sim 35 \%)$ in the process polarized $\mathrm{p}+\mathrm{p} \rightarrow \pi^{1}+$ anything which show interesting structure and appear to be energy independent up to $12 \mathrm{GeV} / \mathrm{c}$. The $\mathrm{p}-\mathrm{p}$ singlet-triplet total cross section difference is large and shows sharp structure as a function of energy between 1 and $3 \mathrm{GeV} / \mathrm{c}$.

$* * * * * * * * * * * * *$ 
ROCKEFELLER UNIVERSITY

New York, New York

Contract No. EY-7.6-C-02-2232

Title of Project: Experimental Physics Laboratory

Person in Charge: R. .Cool.

Scientific and Professional Staff: T. Chapin, Z. Dimcovksi, 0. Fackler, K. Goulianos, A: Rothenberg, S. Segler, H. Sticker, M. Tannenbaum, S. White.

Scope of Work

The high energy physics laboratory of the Rockefeller University is now involved in three major research activities. The first is primarily a study of strong interactions and a search for new phenomena at the highest avallable bombarding energles available at the CERN Intersecting Storage Rings. Together with Columbia University, CERN and Oxford University (CCOR), a new high-field superconducting solenoid has been constructed. The detectors consist of four cylindrical drift-wire chambers for measuring the momenta of charged particles, and about $4 \mathrm{~m}^{2}$ of $\mathrm{Pb}$-glass Cerenkov counters for Identifying $\gamma$-rays and electrons and measuring their energies. The instrument covers a very large solid angle, about $360^{\circ}$ in azimuth and $180^{\circ}$ in polar angle centered at $\pm 90^{\circ}$ with respect to the beam axes. The apparatus is designed to have great flexibility and will be employed with a number of alternative triggering modes to study a wide variety of specific problems. Initially the phenomenon of "jet structure" in the strong interaction and the production of leptons and lepton pairs will be investigated with about 100 times the sensitivity of our initial experiments. The apparatus is now being installed at the ISR.

The second activity is a precision study of the phenomenon of the diffraction dissociation of hadrons. Following completion of a study of protons, a new apparatus (just installed at Fermilab) will be capable of extending the study to pions, kaons, and antiprotons. This important phenomenon, which is responsible for a substantial fraction of the total cross-section, has many extremely interesting regularities and the extension to other hadrons could lead to important new insights. The present apparatus can be used as a trigger to study the decays of the diffractively excited states which have known quantum numbers.

The third activity is the study of neutrino interactions. A study of neutral currents and elastic scattering at BNL with Columbia and Illinois has just been completed. Data to obtain more precise $\nu$ and $\bar{v}$ cross sections and to study the excitation function for muon pairs has been taken at Fermilab with Caltech and Northwestern. 
ROCKEFELLER UNIVERSITY

New York, New York

In addition, extension from 300 to $400 \mathrm{GeV}$ of precision total cross sections of hadrons on protons and deuterons is scheduled during 1977.

Goals and/or Major Results to Date

Major results have been:

1) Discovery at the ISR of the "high transverse momentum" phenomena;

2) First observation of production of the $J / \Psi$ meson at the ISR;

3) First observation of single electrons at the ISR and measurement of the $e^{ \pm} / \pi^{ \pm}$ratios;

4) First observation of strong correlations ("jet-like structure") at the ISR;

5) Discovery that pion and kaon total cross sections rise at Fermilab energies;

6) First measurement of the neutrino-proton elastic cross section at BNL;

T) First observation of the real amplitude for $p-p$ scattering and proof that it rises from negative to positive values at Fermilab energies;

8) Precise and comprehensive measurements of $\mathrm{pp}$ and pd elastic scattering and diffraction dissociation at Fermllab; and

9) Measurements as a function of energy and transverse momentum of the production cross section of $\pi^{\circ}$ and $n^{\circ}$ at the ISR.

$$
* * * * * * * * * * * * *
$$

Title of Project: Theoretical High Energy Physics

Person in Charge: M. Beg, N. Khul, A. Pale

Scientific and Professional Staff: M. Beg, N. Khun, A. Pais, H. Pagels,

A. Sanda, S. Pi, W. Marclano, R. Budny, T. Haglwara

Scope of Work

It is our aim to give the program a broad basis. Thus our recent activities have covered such topics as: 
ROCKEFELLER UNIVERSITY

New York, New York

1) Phenomenology: questions related to charm, heavy leptons, analyses of neutrino reactions and of $\mathrm{e}^{+} \mathrm{e}^{-}$annihilation processes;

2) Gauge theories: study of gauge models, of the CP question in gauge theories, of the pseudo Goldstone phenomenon;

3) Other field theory investigations: short distance operator expansions, soliton type solutions.

4) Chiral symetry and its breakdown; and

5) "Rigorous" results: consequences which follow from rather minimal assumptions such as unitarity, crossing symmetry, etc.

Goals and/or Major Results to Date

Our goal remains to stand ready for new developments, not necessarily to follow every new fad, to keep the composition of the group as versatile as possible. Major results: derivation of a bound (now known as the Pais-Treiman bound) which serves to establish that charm is seen in neutrino reactions at Fermilab; discovery of a new mechanism for generating Pseudo Goldstone bosons; bounds for $\pi^{\circ}$ numbers produced in $\mathrm{e}^{\mathrm{e}}$. annihilation; the most detailed analysis undertaken anywhere of eu events at SPEAR as possible manifestations of heavy leptons. 


\author{
STANFORD UNIVERSITY \\ Stanford, California
}

Contract No. EY-76-S-03-0326, 042

Title of Project: Laser Particle Accelerators

Person in Charge: . R. Pante11

Scientific and Professional Staff: M. Piestrup, G. Rothbart, G. Chen

Scope of Work

The purpose of the program is to investigate the application of lasers to electron acceleration. Lasers can provide very high field strengths which result in large acceleration gradients. For example, in He gas at 80 Torr pressure, breakdown for a 10 psec, $\lambda=6000$ \& laser is $\simeq 10^{9} \mathrm{~V} / \mathrm{cm}$. If the field component for ar.e.1.eration is $1 \%$ of the total field, the corresponding energy gradient is $1 \mathrm{GeV}$ per meter. Another interesting feature of a laser accelerator is that, the current pulses would be extremely short, $10^{-15}-10^{-16}$ seconds, corresponding to a fraction of the period of optical oscillation. To obtain a cumulative increase in electron energy over an extended interaction region it is necessary to obtain wavevector matching between the electrons and the electromagnetic wave; i.e., electron and wave velocities must be equal. We are seeking to accomplish this by using a gas to retard the phase velocity.

\title{
Goals and/or Major Results to Date
}

Electrons will be injected into sodium vapor from a $5 \mathrm{MeV}$ linac, synchronized to arrive simultaneously with the light pulse from a dye laser. Energy change of the electrons will be measured by means of a GeLi detector as a function of the intensity, direction and polarization of the light pulse, and as a function of the sodium vapor pressure.

A monochromator has been designed and is operating in conjunction with the linac to provide $.1 \%$ energy spread with $\pm .5 \mathrm{mrad}$ emittance angle for the electrons. The laser system is being assembled.

The heat pipc for the sodium vapur has been cunslructed; and there is an operating computer program to study electron bunching at optical wavelengths.

$$
* * * * * * * * * * * * * *
$$


STANFORD UNIVERSITY

Stanford, California

Contract No. $\quad$ EY-76-S-03-0326, 046

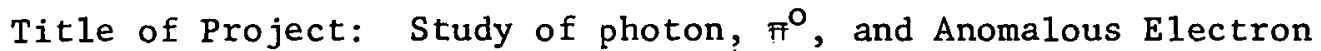
Production in $\mathrm{e}^{+} \mathrm{e}^{-}$. Annihilation

Person in Charge: A. Litke

Scientific and Professional Staff: A. Litke

Scope of Work

We intend to study the production of photons, high energy $\pi^{\circ} \mathrm{s}$, and electrons at the SPEAR electron-positron storage ring. The primary goal is to investigate electron-positron annihilation events in which electrons are produced anomalously, for example from the production and decay of charmed particles or heavy leptons. We also intend to search for the $F$ charmed meson as well as charmed baryons. The experiment will be carried out with the SPEAR magnetic detector facility, supplemented by a combination of lead glass shower counters and spark chambers ("The Lead Glass Wall").

Goals and/or Major Results to Date

A11 of our equipment is installed and operating at SPEAR and we are presently taking data.

$$
* * * * * * * * * *+* * *
$$


STANFORD UNIVERSITY

Stanford, California

Contract No. EY-76-C-03-0850

Title of Project: Experimental Group F

Person in Charge: D. Ritson

Scientific and Professional Staff: D. Ritson, R. Anderson,

D. Gustavson, K. Rich.

\section{Scope of Work}

The work of the group falls into two main catagories:

1) Instrumentation - Design and implementation for experiments. In the past this has included spectrometers such as the SLAC $1.6 \mathrm{GeV} / \mathrm{c}$ spectrometer and the FNAL Single Arm Focussing Spectrometer. Beside conventional hardware, the group has strong capabilities and interests in the software and interfacing areas.

2) Physics - In the past the group has concentrated on strong interaction physics. Work includes:

a) Photoproduction work at SLAC has typically used the $1.6,8$, and $20 \mathrm{GeV}$ SLAC spectrometer facilities in End Station $A$ to study the production of $\pi, k, n, p$, and $\phi$ mesons and more recently the $\Psi, \Psi^{\prime}$ mesons.

b) At FNAL we have studied the elastic and inelastic scattering of $\pi^{ \pm}, \mathrm{K}^{ \pm}$, and $\mathrm{p}^{ \pm}$particles using the FNAL Single Arm Focussing Spectrometer.

Future work may shift emphasis to the field of weak interactions observed via interference effects in $e^{+} e^{-}$collisions at PEP.

\section{Goals and/or Major Results to Date}

1) Recent Results - In collaboration with the University of Wisconsin and the SLAC Spectrometer Facilities Group, we have carried out at SLAC experiments on $\psi, \psi^{\prime}$ and excess lepton photoproduction, and we have determined the variation of the $\Psi$ photoproduction cross section with energy, angle, and atomic number, leading to a determination of the $\psi$-nucleon cross section. 


\section{STANFORD UNIVERSITY \\ Stanford, California}

In collaboration with the Fermilab Single Arm Spectrometer Group using the focussing spectrometer, we have measured elastic and inelastic $\pi^{ \pm}, K^{ \pm} p$, and $p^{ \pm}$scattering for incident energies between 50 and $175 \mathrm{GeV}$.

2) Goals - Our group in collaboration with the University of Wisconsin is presently proposing to build a PEP detector that will concentrate on the leptons, photons, and the energy balance in the final states of $\mathrm{e}^{+} \mathrm{e}^{-}$collisions. We are presently testing prototype chambers at SLAC.

We are also exploring the building of a larger aperture spectrometer for use at SLAC. This spectrometer would be able to measure the two body decays of the heavy mass particles, including $D$ particles.

In the spring of 1977 we will in collaboration with a group from the Argonne National Laboratory use the FNAL spectrometer measuring quasi exclusive channels, such as $\mathrm{K}^{+}$mesons produced in association with $\Sigma^{+}, Y^{++}(1385)$ and $Y^{*+}(1670)$ in $\pi^{+} p$ interactions.

$$
\star * * * * * * * * * * * *
$$


SYRACUSE UNIVERSITY

Syracuse, New York

Contract No. EY-76-S-02-3533

Title of Project: Elementary Particle Theory

Person in Charge: $\mathrm{K}$. Wali

Scientific and Professional Staff: K. Wali, A. Balachandran, J. Schechter, C. Rosenzweig, S. Borchardt, S. Chang, S. Park, G. Campbe11, Jr., J. Kandaswamy, D. Knight, V. Mirelli, A. Stern

Scope of Work

The Syracuse Elementary Particle Theory Group has been actively pursuing several current topics of great interest. The research conducted covers a fairly broad front including both phenomenology and abstract theory.

In the field of strong interactions, hadron spectroscopy, tnponingical considerations in duality schemes and Regge pole and cut descriptions of scattering processes involving arbitrary spin particles are some of the topics under investigation. Special emphasis is being placed on the symmetry aspects of the strong interactions. Dynamical methods which have been successful in the past for SU(3) symmetry, are being extended to higher symmetry schemes such as Su(4).

In the field of weak interactions, there has been a great deal of interest, as elsewhere, in the decay modes of the new particles. Various models which are good candidates for unified theories of weak and electromagnetic interactions are being critically examined in the light of rapidly increasing experimental information.

On a more formal side and also from a long range point of view, quantization of Abelian and non-Abelian 'magnetic' monopole theories are being systematically studied. Towards this end, classical finite energy solutions which have the behavior of solitons are being investigated in model Lagrangians. Topological notions useful in non-Abelian gauge theories in elementary particle physics have been shown to be extremely useful in solid state physirs.

Goals and/or Major Results to Date

While the work in the areas discussed in 'Scope of Work' is currently under progress, some of the results are worth mentioning: 
SYRACUSE UNIVERSITY

Syracuse, New York

1) The Chew-Low method successful in predicting the masses and decay widths of the decuplet in SU(3) seems to work as well in SU(4). Masses of the charmed baryons belonging to the 20-plet $3 / 2^{+}$particles are being calculated.

2) Prediction of life-times of charmed mesons and their various decays.

3) A scheme to include a11 the leptons in an SU(3) octet and consequent decays of recently discovered heavy leptons.

4) Generalization of 't Hooft-Polyakov model:

5) Semiclassical rotationally invariant approximation scheme in monopole theories.

6) Graph-theoretical methods in the analysis of asymptotic behavior of non-Abelian gauge theories.

In spite of a tremendous amount of experimental information concerning elementary particles and their interactions, there are many difficult and puzzling problems for theorists. Our group attempts on the one hand to keep abreast with the current ideas and models, and their critical understanding. Concurrently it also strives to undertake long term projects which are likely to provide a more basic and fundamental understanding of the problems than a current fad or fancy.

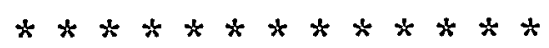


UNIVERSITY OF TENNESSEE

Knoxville, Tennessee

Contract No. EY-76-S-05-3956

Title of Project: Bubble Chamber Studies of Hadron and Photon Interactions

Person in Charge: W. Bugg

Scientific and Professional Staff: W. Bugg, G. Condo, E. Hart, T. Handler

Scope of Work

Currently our group is involved in three classes of high energy physics experiments. The first consists of FNAL hybrid bubble chamber experiments (154 and 299) from which substantial physics has already been obtained. The hybrid system has, for example, proved capable of separating two-prong, inelastic and elastic events in $147 \mathrm{GeV} / c \pi^{-} p$ interactions where it is found that the inelastic events are due predominantly to diffractive dissociation of the beam or target particles. In the second class, measurement continues on two large conventional bubble chamber experiments a 1.5 million picture $8 \mathrm{GeV} / \mathrm{c} \pi^{-} \mathrm{p}$ exposure in collaboration with MIT and Tohoku (Japan), and a half-million picture $\pi^{+} d(15 \mathrm{GeV} / \mathrm{c})$ exposure with Florida State University. Early indications from the former experiment suggest the existence of a resonance in the $S$ region $(M \sim 1920 \mathrm{MeV}, \Gamma \sim 100 \mathrm{MeV}$ ) which cannot be explained as a reflection from nucleon diffractive dissoctation processes. An example of the third class is an experiment that has recently been initiated on a stopping $\bar{p}$-deuterfum exposure obtained at ANL in the 12' chamber.

Goals and/or Major Results to Date

The goal is to test the conclusions previously reported by the Syracuse University group which has bearing on the existence of bound or nearly bound $\mathrm{N} \overline{\mathrm{N}}$ states.

Future efforts will be devoted to carrying through these experiments.

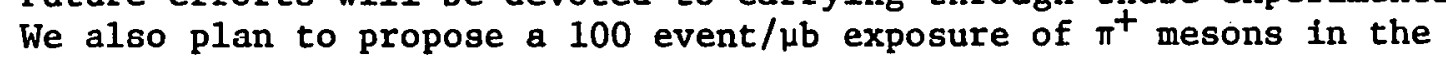
$8 \mathrm{GeV} / \mathrm{c}$ range in the SLAC 40" dcutcrium bubble chamber hybrid facility to study backward charge exchange processes.

$* * * * * * * * * * * * * *$ 
UNIVERSITY OF TEXAS

Austin, Texas

Contract No.

EY-76-S-05-3992

Title of Project: Center for Particle Theory

Person in Charge: E.C.G. Sudarshan and Yuval Ne'eman

Scientific and Professional Staff: A. Bohn, C. Chiu, D, Dicus, A. Gleeson, T.A. Griffy, E.C.G. Sudarshan, Y. Ne'eman, Jong-Ping Hsu, J. Lemmon, T. Sherry, D. Tow

\section{Scope of Work}

The Center for Particle Theory at the University of Texas at Austin is responsible for investigations in the fundamental structure of matter. The staff of the Center consists of a group of experienced particle physicists, whose range of interests spans the entire spectrum of present theoretical elementary particle investigations.

In particular, the group is carrying out fundamental studies ranging from the quantum theory of measurement to the use of sophisticated associative algebraic techniques for the development of the phenomenology of fundamental interactions. Several members of the staff are actively engaged in the development of the mathematical formalism of both an algebraic and field theoretic description of the techniques of supersymmetries. Recent developments of gauge theories of weak interactions and their possible extension is being actively pursued and will continue into the coming year. The application of these new developments to the fu11 range of testable consequences in both particle physics and astrophysics is being carried out.

The development of a relativistically consistent theory of superdense matter is being studied and this study will continue. The application of these methods to problems in nuclear physics and elementary particle physics is projected, along with their continued application to astrophysical situations. New phenomenologies recently developed at the high energy laboratories are being examined and extended by the group at Texas. Overall, the program is one that is both comprehensive in its interests and yet specific in the developments. Recent excitements associated with elementary particle phenomenon are all clearly reflected in the activities of this group. 
UNIVERSITY OF TEXAS

Austin, Texas

\section{Goals and/or Major Results to Date}

The group in the Center for Particle Theory at the University of Texas at Austin has been responsible for significant contributions in almost all areas of particle physics. The members of the staff at the Center are internationally recognized for their work in finite and constrained field theories. They have produced the definitive investigations of the possibilities of indefinite metric and high spin field theories. In the area of the application of the group theoretic methods to particle theory phenomenology, they are wel1-known for their development of dynamical symmetries. They are the leading institution for the application of associative algebras and graded Lie algebras to the problems of the spectra and decay states of the elementary particles. The development of a consistent theory of superdense matter has provided for the first suitable explanation of pulsar phenomenon as manifestations of neutron star structure. Methods of hydrodynamic and thermodynamic fluid models of hadrons were originally developed at Texas and have been applied extensively here and at other institutions.

$$
* * * * * * * * * * * * *
$$




\section{TUFTS UNIVERSITY \\ Medford, Massachusetts}

Contract No. EY-76-C-02-3023

Title of Project: High Energy Physics (Photon Group)

Person in Charge: R. Milburn

Scientific and Professional Staff: R. Milburn, J. Rutherford, D. Quinn, A. Stottlemyer, R. Thornton, W. Oliver, M. Shupe

Scope of Work

The principal activity of the Group during 1976 has been the analysis of data from an experiment at the Cornell Electron Synchrotron to measure the cross sections for wide-angle proton Compton scattering on protons and for neutral-pion photoproduction at primary energies 2-6 GeV and four-momentum transfers (squared) in the range 0.71-4.29 $(\mathrm{GeV} / \mathrm{c})^{2}$, corresponding to center-of-mass angles in the range 45$128^{\circ}$. This analysis is expected to be completed in the early fall of 1976, to yield data which bear on the legitimacy of parton, dimensional-counting and other models of hadronic structure and interactions. (This experiment is in collaboration with physicists from University of Massachusetts-Amherst, MIT, and Corne11).

Work is continuing on the exploration of technical laser and accelerator developments which could enable the production via the backscattered Compton photon technique of energetic and polarized gamma rays in the $1-20 \mathrm{GeV}$ range at high repetition rate. Such a beam is essential for track chamber studies of polarized photoproduction at . the sub-microbarn level.

A search for charmed mesons and baryons photoproduced at $10 \mathrm{GeV}$ was concluded; none were found at the $100 \mathrm{nb}$ level.

Work has been initiated to adapt the group's $\mathrm{Pb}-\mathrm{glass}$ Cerenkov hodoscope to permit discrimination of electrons from heavy hadronic backgrounds, this in relation to a proposal being presented to SLAC (in collaboration with Dr. Z.M. Ma of Michigan State University) for a streamer chamber study of direct production by hadrons of large $\mathrm{p}_{\mathrm{T}}$ leptons and other states in association with $\mathrm{K}^{-}$.

Goals and/or Major Results to Date

1) Results: extension of proton Compton scattering and pi-0 photoproduction data to a hitherto unexplored region of large center-of-mass angles at high energies. 
TUFTS UNIVERSITY

Medford, Massachusetts

2) Future Goals:

a) Measurement of phi-meson production mechanisms and $\mathrm{K}^{-}$ associated lepton in. $\pi^{+} p$ interactions at $15 \mathrm{GeV} / \mathrm{c}$ (proposal to SLAC in preparation: Michigan State University collaboration).

b) Extension of polarized "laser beam" techniques to permit high repetition rate track chamber experiments at SLAC, and possibly also ref.

$$
\text { * }
$$

Title of Project: Bubble Chamber Sroupe

Person in Charge: J. Schneps

Scientific and Professional Staff: J. Canter, F. Dao, A. Mann, J. Schneps, E. Gelfand, H. Wald

Scope of Work

In a $15 \mathrm{GeV} / \mathrm{c}$ pp exposure in the BNL 80-inch chamber we are searching for charmed particle production. We detect hadronic decays into $V^{\circ}$ plus charged prongs and semi-leptonic decays into $\mathrm{V}^{\circ} \mathrm{e}(v)$. The electrons are detected using a tantalum plate in which they shower. Charged and total particle multiplicities are also being measured. In a pd exposure at $300 \mathrm{GeV} / \mathrm{c}$ in the Fermilab 30" chamber we are studying charged-particle multiplicities, and single particle distributions and excited states from neutron target fragmentation. The primary objectives in a $300 \mathrm{GeV} / \mathrm{c}$ pp experiment in the Fermilab 15-foot chamber are the dependence of neutral particle production on charged multiplicity and a measure of the correlations $f_{2}^{+0}$ and $f_{2}^{O O}$ for neutral pions. We have used $28.5 \mathrm{GeV} / \mathrm{c} \mathrm{pp}$ interactions from the BNL ARGO group to study Bose-Einstein statistical correlations among emitted pions. In the million picture $6.5 \mathrm{GeV} / \mathrm{c} \mathrm{K}^{-} \mathrm{p}$ experiment, in the 12-foot chamber, we are using the zero-prong plus $V$ topology to attempt to isolate narrow width neutral mesons in the reaction $\mathrm{K}^{-} \mathrm{p} \rightarrow \Lambda^{\circ}+\left(\mathrm{MM}=\pi^{\circ}, n, \omega, \eta^{\prime}, \phi, f,-\ldots\right)$. In $2.9 \mathrm{GeV} / \mathrm{c}$ $K^{-} d$ we have searched for exotic states from the reaction $\mathrm{K}^{-} \mathrm{n} \rightarrow \Sigma^{-} \pi^{-} \pi^{+}\left(\pi^{\circ}\right)$. In mid 1977 we will begin a study of vd interactions in the Fermilab 15-foot chamber. We intend to investigate elastic form factors, $\Delta(1236)$ production, $|\Delta I|=1$ and $\Delta S=\Delta Q$ rules, un and up cross-sections, details of hadronic final states, and strange and charmed particle production. 
TUFTS UNIVERSITY

Medford, Massachusetts

Goals and/or Major Results to Date

We have found a correlation in the direct production of electrons (positrons) with $\mathrm{K}^{\mathbf{O}}$ mesons in $15 \mathrm{GeV} / \mathrm{c} \overline{\mathrm{p}}$ interactions. The events could be interpreted as the decays of charmed particles which are pair produced in these interactions. We also find evidence for a $\Delta 3 \pi$ hadronic state at $2.4 \mathrm{GeV} / \mathrm{c}$ which might be interpreted as a charmed baryon decay. In $300 \mathrm{GeV} / \mathrm{c}$ pd interactions we find the pn multiplicity distribution tends to be shifted from the pp by one unit at low multiplicities, whereas at higher multiplicities the two are interleaved. The determination of $\mathrm{f}_{2}^{\text {oo }}$ will be made only after the $300 \mathrm{GeV} / \mathrm{c}$ pp film has been triple-scanned for $\gamma^{\prime} \mathrm{s}$. In the $6.5 \mathrm{GeV} / \mathrm{c} \mathrm{K} \mathrm{K}^{-} \mathrm{p}$ film results on inclusive $\mathrm{V}^{\circ}$ processes have been obtained and progress toward full analysis of 0 -prong plus $V$ is being made. Statistical correlations between like pions emitted from $28.5 \mathrm{GeV} / \mathrm{c}$ pp interactions have been observed and the size of the interaction is determined to be 1.3 fermis.

$$
\star \star \star * * * * * * * * * * * *
$$




\section{UNIVERSITY OF WASHINGTON \\ Seattle, Washington}

Contract No: $\quad$ EY-76-S-06-2225,0027

Title of Project: High Energy Physics Studies of Particle Interactions and the Search for "new" Particles

Person in Charge: J. Lord

Scientific and Professional.Staff: J. Florian; R. Wilkes.

Scope of Work

Experimental investigations of the production of "charmed" and other short-lived particles which may be produced in deep-inelastic muon interactions. The techniques used in the experiments involve the utilization of a hybrid system of nuclear track emulsions, drift chambers, and a muon spectrometer. The experiment involves $150 \mathrm{GeV}$ muons at Fermilab and is designated as experiment E-382.

Additional experiments involve the study of intranuclear cascade processes in elements from $\mathrm{A}=12$ to 184 . The targets are heavy element granules in emulsions.

Goals and/or Major Results to Date

Our experiments were the first at Fermilab to show that the multiplicity of pion production in elements as heavy as $A=184$ was considerably less than predicted by theory. 'It was also shown that the rapidity distribution in the forward direction was very nearly independent of $A$ from $A=1$ to $A=184$. 
WAYNE STATE UNIVERSITY

Detroit, Michigan

Contract No. EY-76-S-02-2302

Title of Project: Quantum Theory of Fields

Person in Charge: S. Gupta

Scientific and Professional Staff: S. Gupta, D. Barua

Scope of Work

The research program is devoted to the development of the basic. theories of electromagnetic,. strong, weak, and gravitational interactions as well as their applications to fundamental physical processes. Problems currently under investigation deal with the quantization of gauge fields, renormalizable theories of weak and strong interactions, anomalies in weak and nonweak interactions, mass splittings and mixing angles of hadrons, symmetrybreaking effects in decay processes, and treatment of higher-spin fields with idenfinite metric.

Goals and/or Major Results to Date

In a forthcoming paper entitled "Quantization of Gauge Fields without the Path-Integral. Formalism". by S. N: Gupta, an elegant formalism for the quantization of gauge fields is developed within the framework of standard field theory. This paper does not make any use whatsoever of the obscure and complicated path-integral formalism employed by earlier authors.

$$
* * * * * * * * * * * * * *
$$

